\title{
SPECIAL ISSUE: ABSTRACTS FROM THE 2008 ISSOL MEETING
}

\section{INTRODUCTION TO THE SPECIAL ISSUE}

This issue of Origins of Life and Evolution of Biospheres contains the abstracts of the scientific contributions presented at the 2008 ISSOL Meeting, which was held in Florence (Italy) on 24-29 August, 2008.

The Symposium's main objectives were to bring together scientists working in different areas of the study of the origin and early evolution of life, to stimulate discussion on this fundamental process and to have an appraisal of the most recent advances in this multidisciplinary field that combines research from space sciences and astrophysics, to chemistry, geology, paleontology, genomics, molecular biology, history and philosophy of science, among others.

The meeting was attended by about 350 scientists from all over the world, and more than 310 presentations were given, including 260 posters.

This volume collects almost all the contributions, which are an up-to-date account of the state of the knowledge on this exciting area of scientific and educational pursuits.

It is with great pleasure that I acknowledge the contributions of different authors in assuring the prompt publication of the OLEB Special Issue.

I would also like to express my thanks to the Editor of OLEB, Alan W. Schwartz, and Springer for the publication of the Proceedings.

Enzo Gallori

University of Florence

President of the Local Organizing Committee 


\title{
Invited Lectures
}

\section{Search for Potentially Primordial Genetic Systems}

\author{
Ramanarayanan Krishnamurthy
}

The Department of Chemistry at The Scripps Research Institute 10550 North Torrey Pines Road, MB16, La Jolla, CA-92037, USA

Extensive base-pairing studies of oligonucleotides consisting of canonical bases tagged to a variety of cyclic sugar-phosphate backbones - conducted in the context of work toward an etiology of the structure type of the natural nucleic acids - have led to a broadening of the scope of investigations to include informational oligomer systems that are not confined to typical sugar-backbones and canonical bases.

The lecture will present some recent results: the base-pairing properties of a series of acyclic backbone derived oligomeric systems tagged with alternative heterocycles as recognition elements.

E-mail: rkrishna@scripps.edu

\section{The Formation of Planetary Systems}

Alan P. Boss

Carnegie Institution, Washington DC, USA

Planetary systems form out of the leftovers of the star formation process. Dense interstellar clouds of gas and dust collapse under their self-gravity to form central protostars orbited by rotationally supported, flattened disks in which planets later form. The disk that formed the Solar System is called the solar nebula. Terrestrial planets form by the slow process of collisions and sticking between increasingly larger dust grains, pebbles, boulders, and mountains of rock and ice termed planetesimals. Km-size planetesimals are large enough to grow by gravitationally deflecting bodies that might otherwise not collide with them, leading to a period of runaway growth to lunar-sized planetary embryos. The final phase of terrestrial planet formation involves giant impacts between the protoplanets and planetary embryos and requires on the order of 100 million years.

While there is a general consensus about the formation of terrestrial planets, two very different mechanisms have been proposed for the formation of the gas and ice giant planets. The conventional explanation for the formation of gas giant planets, core accretion, presumes that a gaseous envelope collapses upon a roughly ten Earth-mass, solid core of rock and ice that was formed by the collisional accumulation of planetary embryos orbiting in the solar nebula. The more radical explanation, disk instability, hypothesizes that the gaseous portion of the nebula underwent a gravitational instability, leading directly to the formation of self-gravitating clumps, within which dust grains coagulated and settled to form cores. Core accretion appears to require several million years or more to form a gas giant planet, implying that only relatively long-lived disks would form gas giants. Disk instability, on the other hand, is so rapid (forming clumps in thousands of years), that gas giants could form in even the shortest-lived disks. Terrestrial planets seem to be likely to 
form under either scenario for giant planet formation, though the likelihood does depend strongly on the orbital properties of the giant planets in the system.

Core accretion has difficulty in explaining the formation of the ice giant planets, unless two extra protoplanets are formed in the gas giant planet region and thereafter migrate outward. An alternative mechanism for ice giant planet formation has been proposed, based on observations of protoplanetary disks in the Orion nebula cluster and Eta Carina starforming region: disk instability leading to the formation of four gas giant protoplanets with cores, followed by photoevaporation of the disk and gaseous envelopes of the protoplanets outside about $10 \mathrm{AU}$ by ultraviolet radiation from nearby massive stars, producing ice giants. In this scenario, Jupiter survives unscathed, while Saturn is a transitional planet. The ultraviolet fluxes photoevaporate the outer disk, freezing the orbits of the giant planets, and converting the outer gas giants into ice giants.

Because most stars form in regions of high-mass star formation, if this alternative scenario is appropriate for the formation of the Solar System, extrasolar planetary systems similar to our own may then be commonplace. This heretical idea also offers a head start on the formation of prebiotic molecules necessary for the origin of life, as well as a natural explanation for thermally processing primitive materials (chondrules and refractory inclusions) found in meteorites, large scale radial transport of refractories and ices, mixing and homogenization of initially heterogeneous short-lived radioactivities, and transport of stable oxygen isotopes inward from the outer disk surface, as required by cosmochemical constraints on the formation of the Solar System.

E-mail: boss@dtm.ciw.edu

\section{Gas-Phase Prebiotic Chemistry in the Solar System: How and Where}

\section{Nadia Balucani}

\section{Dipartimento di Chimica, Università degli Studi di Perugia, Perugia, Italy}

In the sequence of steps which are believed to have led from elementary particles to the emergence of life, an important one is certainly the formation of simple prebiotic molecules from parent species abundant in the Universe. The aggregation of $\mathrm{H}, \mathrm{O}, \mathrm{N}, \mathrm{C}$ (and other elements) atoms into molecules and the subsequent chemical evolution are occurring also now in the Universe, as witnessed by the identification of more than one hundred molecules in the interstellar medium (encompassing also prebiotic molecules such as glycolaldehyde, formamide and, tentatively, glycine) and by the gas-phase chemical evolution of the atmospheres of several solar objects like Titan. Simple as they might seem compared to other processes of relevance in astrobiology, the formation mechanisms of many of the observed gaseous prebiotic molecules and radicals are far from being understood.

In this contribution, the focus will be on the gas-phase chemical evolution of planetary atmospheres and cometary comae, the gaseous environments of our Solar System where gaseous organic molecules have been observed. Similarly to the atmosphere of Earth, the atmospheres of the other planets (or satellites, like Titan) can be described as giant photo-reactors, where the energy deposited mainly by solar photons, but also by cosmic rays and other energetic particles, drives a complex gas-phase chemistry. In this specific context, gas-phase neutral-neutral reactions are expected to play a dominant role. A thorough characterization of the chemical evolution of planetary atmospheres relies on a multi-disciplinary approach: (1) observations allow us to identify the molecules and their number densities as they are nowadays; (2) the 
chemistry which lies behind their formation starting from atoms and simple molecules is accounted for by complex reaction networks; (3) for a realistic modeling of such networks, a number of experimental parameters are needed and, therefore, the relevant molecular processes should be fully characterized in laboratory experiments. A survey of the available literature reveals, however, that much information is still lacking if it is true that only a small percentage of the elementary reactions considered in the models have been characterized in laboratory experiments. New experimental approaches to characterize the relevant elementary reactions in laboratory are presented and the implications of the results are discussed.

E-mail: nadia.balucani@unipg.it

\title{
The Evolution of the Primitive Atmosphere
}

\author{
James F. Kasting
}

\section{Department of Geosciences, Penn State University, University Park, PA 16802}

Environmental conditions on the early Earth are important for both the origin and the early evolution of life. Two variables are of particular significance: (1) the atmospheric redox state, and (2) the mean surface temperature. Most recent models of Earth's prebiotic atmosphere (Walker, 1977; Kasting, 1993) suggest that it was weakly reduced, with $\mathrm{N}_{2}$ and $\mathrm{CO}_{2}$ dominating over $\mathrm{NH}_{3}$ and $\mathrm{CH}_{4}$. Some $\mathrm{CH}_{4}$ may have been present, however (Hashimoto et al., 2007), particularly if hydrogen escape was relatively slow (Tian et al., 2005). Ongoing work should help to resolve the hydrogen escape question and may shed light on whether a more highly reduced atmosphere could have existed.

The climate of the early Earth is also controversial. Despite the faintness of the young Sun, the early Earth appears to have been warm, or perhaps even hot. Taken at face value, oxygen and silicon isotopes in ancient cherts imply a mean surface temperature of $70( \pm 15)^{\circ} \mathrm{C}$ at $3.3 \mathrm{Ga}$ (Knauth and Lowe, 2003; Robert and Chaussidon, 2006). Ancient carbonates also yield high Precambrian surface temperatures (Shields and Veizer, 2002), as does a recently published analysis of the thermal stability of proteins which are inferred to be ancient (Gaucher et al., 2008). This evidence for hot early surface temperatures must be weighed against the previously mentioned dimness of the young Sun, as well as geomorphic evidence for glaciation at 2.9, 2.4, and 0.6-0.7 Ga. Climate models with high $\mathrm{CO}_{2}$ and $\mathrm{CH}_{4}$ concentrations can potentially explain hot climates, but can they explain climates that transition from hot to cold, and back again, multiple times? Such models must also account for the well documented correlation between the rise of $\mathrm{O}_{2}$ at $2.4 \mathrm{Ga}$ and the Paleoproterozoic glaciations which occurred at that same time. Some of the secular variation in oxygen isotope ratios may be accounted for by changes in seawater isotopic composition (Kasting et al., 2006), although that interpretation remains controversial and cannot account for the observed variation during the Phanerozoic (Came et al., 2007). When all the arguments are weighed, the early Earth appears to have been warm, rather than hot, but more work remains to reconcile the different pieces of evidence.

Came, R. E., Eiler, J. M., Veizer, J., Azmy, K., Brand, U., and Weidman, C. R. (2007). Coupling of surface temperatures and atmospheric $\mathrm{CO}$ concentrations during the Palaeozoic era. Nature, 449: 198-201.

Gaucher, E. A., Govindarajan, S., and Ganesh, O. K. (2008). Palaeotemperature trend for Precambrian life inferred from resurrected proteins. Nature, 451: 704-707. 
Hashimoto, G. L., Abe, Y., and Sugita, S. (2007). The chemical composition of the early terrestrial atmosphere: Formation of a reducing atmosphere from CI-like material. Journal of Geophysical Research-Planets, 112: E05010.

Kasting, J. F. (1993). Earth's early atmosphere. Science, 259: 920-926.

Kasting, J. F., Howard, M. T., Wallmann, K., Veizer, J., Shields, G., and Jaffres, J. (2006). Paleoclimates, ocean depth, and the oxygen isotopic composition of seawater. Earth Planet. Sci. Lett., 252: 82-93.

Knauth, P. and Lowe, D. R. (2003). High Archean climatic temperature inferred from oxygen isotope geochemistry of cherts in the 3.5 Ga Swaziland Supergroup, South Africa. GSA Bull., 115: 566-580.

Robert, F. and Chaussidon, M. (2006). A palaeotemperature curve for the Precambrian oceans based on silicon isotopes in cherts. Nature, 443: 969-972.

Shields, G. and Veizer, J. (2002). Precambrian marine carbon isotope database: version 1.1. Geol. Geochem. Geophys., 3: June 6.

Tian, F., Toon, O. B., Pavlov, A. A., and De Sterck, H. (2005). A hydrogen rich early Earth atmosphere. Science, 308: 1014-1017.

Walker, J. C. G. (1977). Evolution of the Atmosphere. Macmillan, New York.

E-mail: kasting@essc.psu.edu

\section{Synthesis of Nucleic Acid Components}

\section{Raffaele Saladino}

Agrobiology \& Agrochemistry Department, University of Tuscia, Via S, Camillo de Lellis s.n.c., 01100 Viterbo, Italy

Plausible scenarios for the origin of life entail the robust prebiotic synthesis of informational polymers by condensation of simple chemical precursors (Saladino and Di Mauro, 2005). Among the chemical precursors taken into consideration, two related compounds, hydrogen cyanide $(\mathrm{HCN})$ and formamide $\left(\mathrm{NH}_{2} \mathrm{COH}, \mathbf{1}\right)$, were matter of thorough analyses (Saladino and Di Mauro, 2004; Saladino and Di Mauro, 2006; Saladino and Di Mauro, 2007). The attention for these two compounds is mainly due to their ability to synthesize nucleic bases and amino acids under experimental conditions relatively mild and coherent with those existing on the primitive Earth. Noteworthy, formamide is the only chemical precursor able to synthesize at the same time, in addition to some amino acid derivatives, both purine and pyrimidine nucleic bases (Ciciriello, Saladino and Di Mauro, 2007; Costanzo, Saladino and Di Mauro, 2007; Ciciriello, Saladino and Di Mauro, 2008). Here we show, in agreement with the seminal hypotheses of Bernal (Bernal, 1951) and Cairns-Smith Cairns-Smith 1992), that the prebiotic chemistry of formamide is finely tuned by the presence of different metal oxides and minerals in the reaction mixture, thus modelling the microenvironment of the primitive Earth. These compounds can act as catalysts for condensation processes, enhancing the concentration of the reactant and preserving newly formed biomolecules from chemical and photochemical degradation. Moreover, the elemental composition of the minerals used as catalysts plays a major role in the selectivity of the syntheses of nucleic bases catalyzing the in situ decomposition of formamide to other chemicals potentially useful for the construction of both purine and pyrimidine scaffolds. Taken together, these procedures suggest novel scenarios for the molecular evolution of life on the primitive 
Earth and may provide a chemical clue to the evaluation of the plausible emergence of extraterrestrial forms of life.

J. D. Bernal, The Physical Basis of Life, Routledge and Kegen Paul, (1951) London.

G. Cairns-Smith in Possibile Role for Minerals in Early Organisms, J. Tran Tharh Van, J. C. Mounolou, J. Schneider, C. McKay, Eds., Editions Frontiéres, Gif-sur-Yvette, France, (1992), 119-132.

F. Ciciriello, G. Costanzo, S. Pino, C. Crestini, R. Saladino, E. Di Mauro (2008) Biochemistry 47(9), 2732-2742.

G. Costanzo, R. Saladino, C. Crestini, F. Ciciriello, E. Di Mauro (2007) J. Biol. Chem. 282, 16729-16735.

R. Saladino, C. Crestini, G. Costanzo, E. Di Mauro (2005) Topics in Current Chemistry, Ed. P. Walde, Springer-Verlag Berlin Heidelberg.

R. Saladino, C. Crestini, F. Ciciriello, G. Costanzo, R. Negri, E. Di Mauro (2004) Astrobiology: Future Perspective, P. Ehrenfreund ed., Netherlands 393-413.

R. Saladino, C. Crestini, F. Ciciriello, G. Costanzo, E. Di Mauro (2006) Orig. Life Evol. Biosph. 36, 523.

R. Saladino, C. Crestini, F. Ciciriello, G. Costanzo, E. Di Mauro (2007) Chemistry \& Biodiversity 2007, 4, F. Ciciriello, G. Costanzo, C. Crestini, R. Saladino, E. Di Mauro, (2007) Astrobiology, 7, 616-630.

E-mail: saladino@unitus.it

\section{Evolution of the Genetic Code and the Earliest Proteins}

Edward. N. Trifonov

University of Haifa, Israel, and Masaryk University, Brno, Czech Republic

Reconstruction of evolutionary history of the genetic code (Trifonov, 2000) on the basis of consensus temporal order of engagement of amino acids in early evolution, provides a powerful tool for further reconstruction of early molecular events. In particular, the binary code of protein sequences has been suggested by the evolutionary chart of the codons, and confirmed (Gabdank et al., 2006). The binary sequences (of Alanine type and Glycine type residues) represent possible ancestral forms of modern 20-letter alphabet sequences. Oligopeptides that are found in proteomes of every prokaryote (omnipresent elements), that are likely to represent the sequences from last common ancestor, in their binary form all fit to a unique Aleph-Beth Prototype sequence, that corresponds to ATPbinding and ATPase modules of modern ABC transporters. The ancestral forms of these two modules are not only identical, but also "complementary", that is, they apparently have been encoded in opposite strands of the same duplex gene. The Prototype has mosaic structure, being built of single point change derivatives of primordial (Gly) 7 and $(\text { Ala })_{7}$ peptides.

Gabdank, I., Barash, D., Trifonov, E. N., Tracing ancient mRNA hairpins. J Biomol Str Dyn 24, 163-170 (2006)

Trifonov, E. N., Consensus temporal order of amino acids and evolution of the triplet code. Gene 261, 139-151 (2000)

E-mail: trifonov@research.haifa.ac.il 


\section{Asymmetric Autocatalysis and the Origins of Homochirality}

\section{Kenso Soai}

Department of Applied Chemistry, Tokyo University of Science, Kagurazaka, Shinjuku-ku, Tokyo 162-8601, Japan,

The automultiplication and homochirality are two characteristic features of life. The establishment of the systems of automultiplication and the homochirality of compounds had been the prerequisite for the chemical origins of life. Several theories have been proposed for the possible origins of chirality such as circularly polarized light (CPL), chiral inorganic crystals, spontaneous absolute asymmetric synthesis, and chiral crystals of achiral organic compounds, However, enantioenrichments induced by these proposed origins of chirality have been very low, and the relationship has not been clear between the low enantioenrichments induced by the proposed mechanisms and the high enantioenrichment of biomolecules.

We report asymmetric autocatalysis with amplification of chirality. Pyrimidyl alkanol works as an asymmetric autocatalyst in the addition of diisopropylzinc to pyrimidine-5carbaldehyde. The initial very low (ca. $0.00005 \%$ ee) enantioenrichment of asymmetric autocatalyst amplifies significantly to near enantiopure $(>99.5 \%$ ee) by three consecutive asymmetric autocatalysis also with significant multiplication factor of the amount (ca. 630,000 times) (Soai, 2004. Soai and Kawasaki, 2008).

The tiny enantioenrichments induced by right or left handed CPL, chiral inorganic crystals such as $d$ and $l$-quartz, sodium chlorate, cinnabar, and chiral crystals of achiral organic compounds are correlated successfully to the high enantioenrichments by asymmetric autocatalysis. CPL and chiral crystals serve as chiral initiators of asymmetric autocatalysis and gave the highly enantioenriched pyrimidyl alkanol with the absolute configuration correlated to those of the chiral initiators. Spontaneous absolute asymmetric synthesis is possible with the asymmetric autocatalysis. Even without adding chiral initiator, i.e., the reaction between pyrimidine-5-carbaldehyde and diisopropylzinc, the enantioenriched pyrimidyl alkanol with either $S$ or $R$ configuration are formed. Asymmetric autocatalysis is a powerful method for chiral discrimination and the elucidation of the mechanism of the reaction (Kawasaki et al., 2006. Sato et al., 2007. Lutz et al., 2008).

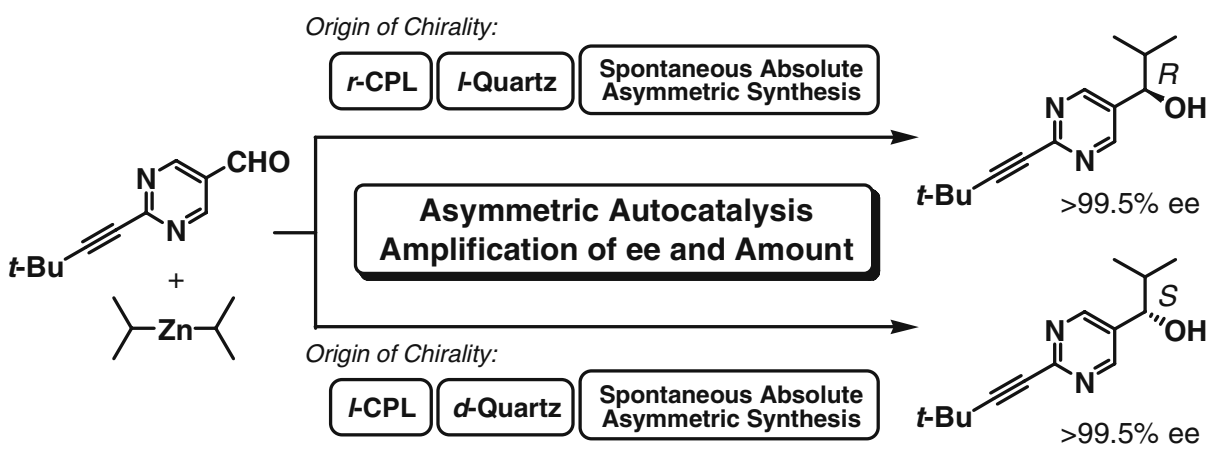

Lutz, F., Igarashi, T., Kinoshita, T., Asahina, M., Tsukiyama, K., Kawasaki, T., and Soai, K. (2008). Mechanistic Insights in the Reversal of Enantioselectivity of Chiral Catalysts by Achiral Catalysts in Asymmetric Autocatalysis. J. Am. Chem. Soc., 130:2956-2958. 
Kawasaki, T., Hatase, K., Fujii, Y., Jo, K., Soai, K. and Pizzarello, S. (2006). The Distribution of Chiral Asymmetry in Meteorites: An Investigation Using Asymmetric Autocatalytic Chiral Sensors. Geochim. Cosmochim. Acta, 70:5395-5402.

Sato, I., Ohgo, Y., Igarashi, H., Nishiyama, D., Kawasaki, T. and K. Soai, (2007). Determination of Absolute Configurations of Amino Acids by Asymmetric Autocatalysis of 2-Alkynylpyrimidyl Alkanol as a Chiral Sensor. J. Organomet. Chem., 692:1783-1787.

Soai, K. (2004). Asymmetric Autocatalysis, Absolute Asymmetric Synthesis and Origin of Homochirality of Biomolecules. In: G. Pályi, G., Zucchi, C., and Caglioti, L. (Ed.), Progress in Biological Chirality, Chap. 29, Elsevier, Oxford, pp. 355-364.

Soai, K. and Kawasaki, T. (2008). Asymmetric Autocatalysis with Amplification of Chirality. In: Soai, K. (Ed.), Topics in Current Chemistry: Amplification of Chirality, Springer, Berlin.

E-mail: soai@rs.kagu.tus.ac.jp

\section{Self-Sustained Replication of RNA Enzymes}

Gerald F. Joyce

The Scripps Research Institute, La Jolla, CA, USA

Our research efforts have focused on the development of catalytic RNA molecules that are relevant to the establishment and maintenance of RNA-based life on the primitive Earth (Joyce, 2002). Especially critical is the ability of RNA to catalyze the replication of RNA molecules, thereby enabling the self-sustained evolution of RNA. Employing methods of in vitro evolution, our laboratory and others have developed a variety of RNA enzymes that catalyze the RNA-templated joining of RNA (Bartel and Szostak, 1993; Robertson and Ellington, 1999; Jaeger, et al., 1999). One such enzyme, the R3C ligase (Rogers and Joyce, 2001), was configured so that it could produce additional copies of itself by joining two component oligonucleotides (Paul and Joyce, 2002). It subsequently was converted to a cross-catalytic format whereby two RNA enzymes catalyze each other's synthesis from a total of four oligonucleotides (Kim and Joyce, 2004).

Recently, we optimized the activity of the cross-replicating RNA enzymes so that they can undergo self-sustained exponential amplification in the absence of proteins. In one such experiment, the RNA enzymes underwent billion-fold amplification in $30 \mathrm{~h}$ at a constant temperature of $42^{\circ} \mathrm{C}$. We have constructed small model populations of crossreplicating RNA enzymes that undergo self-sustained exponential amplification within a common reaction mixture. In these experiments we have observed selection of the fittest replicators, depending on the choice of reaction conditions. Our current efforts are focused on understanding the determinants of replication efficiency and fidelity so that we can construct more complex populations of exponentially amplifying RNAs. This would allow self-sustained Darwinian evolution to occur within a synthetic genetic system.

Bartel, D. P. and Szostak, J. W. (1993). Isolation of new ribozymes from a large pool of random sequences. Science 261:1411-1418.

Jaeger, L., Wright, M. C., and Joyce, G. F. (1999). A complex ligase ribozyme evolved in vitro from a group I ribozyme domain. Proc. Natl. Acad. Sci. USA 96:1471214717.

Joyce, G. F. (2002). The antiquity of RNA-based evolution. Nature 418:214-221. 
Kim, D.-E. and Joyce, G. F. (2004). Cross-catalytic replication of an RNA ligase ribozyme. Chem. Biol. 11:1505-1512.

Paul, N. and Joyce, G. F. (2002). A self-replicating ligase ribozyme. Proc. Natl. Acad. Sci. USA 99:12733-12740.

Robertson, M. P. and Ellington, A. D. (1999). In vitro selection of an allosteric ribozyme that transduces analytes into amplicons. Nature Biotechnol. 17:62- 66 .

Rogers, J. and Joyce, G. F. (2001). The effect of cytidine on the structure and function of an RNA ligase ribozyme. RNA 7:395-404.

E-mail: gjoyce@scripps.edu

\section{Cosmochemical Evolution and the Origins of Life: A Tribute to Joan Oró}

\section{Sandra Pizzarello}

Arizona State University, Tempe AZ 85287-1604 USA

Joan (John) Oró was an enthusiastic and eclectic exobiologist who, since the early days of the discipline, promoted the idea of cosmochemical evolution as a possible precursor to terrestrial life (Oró, 1961). The idea also made him a pioneer in meteoritic studies, as he recognized the importance of natural sample analyses towards the understanding and modeling of life's origins. This lecture in his honor will tell of new types of meteorites and the advances that their analyses have brought to our knowledge of prebiotic extraterrestrial chemistry. Carbonaceous meteorites provide a detailed record of the organic materials that can be synthesized in abiotic environments. These have been shown to be complex and to have structures as varied as kerogen-like macromolecules and simpler soluble compounds, e.g., amino acids and hydrocarbons (Pizzarello et al., 2006). Meteorite organics display an overall molecular and isotopic diversity that points to synthetic pathways in a variety of chemical regimes, such as exothermic reactions in the cold, hydrogen fractionating interstellar gas phase and aqueous reactions in asteroidal parent bodies. Within this diversity, some meteoritic compounds have been found to be identical to biomolecules, with some of the amino acids displaying the biochemical trait of chiral asymmetry. This, in turn, has suggested that their delivery to the early Earth might have contributed to terrestrial molecular evolution (Pizzarello, 2006). Yet, so far, the study of meteorites has been hindered by the fact that the carbonaceous types are few in recorded falls (only 18 in the last two centuries), are often lost or irreparably altered after their fall and that their soluble organic content degrades with terrestrial exposure (Cronin et al., 1980). This fate may be spared to the stones recovered in Antarctica, where in-falling meteorites are quickly covered by snow, buried within the ice and resurface only when the flowing ice sheets end-up against the obstacle of a mountain. Owing to this unique shelter of the glaciers, American and Japanese scientific expeditions have found here a large number of carbonaceous meteorites, some of which are unspoiled. We will report on the organic composition of two pristine Antarctic meteorites belonging to the Renazzo-type group. These analyses have offered a yet unknown view of the synthetic capabilities and prebiotic potential of extraterrestrial environments, revealing a soluble organic suite made up mainly of water-soluble compounds, with predominant N-containing species and where some of the amino acids display the highest deuterium enrichment ever measured for extraterrestrial molecules by direct analyses. Also the analyses of these meteorites' diastereomer amino acids suggest that their precursor aldehydes carried enantiomeric excesses during the aqueous phase reactions that took place in the meteorites' asteroidal parent bodies (Pizzarello et al., 2008). 
Cronin, J.R., Moore, C.B. and Pizzarello, S. (1980) Amino acids in six CM2 chondrites. Meteoritics, 55: 277.

Oró, J. (1961). Comets and the formation of biochemical compounds on the primitive Earth. Nature, 190: 389-390.

Pizzarello, S. (2006) The chemistry of life's origin: A carbonaceous chondrite perspective. Acc. Chem. Res., 39: 231-237.

Pizzarello, S., Cooper, G.W. and Flynn, G. (2006) in Meteorites and the Early Solar System II, D.S. Lauretta and H.Y. McSween Jr. eds., University of Arizona press, USA pp. 625-651. Pizzarello, S., Huang, Y. and Alexandre, M.R. (2008) Molecular asymmetry in extraterrestrial chemistry: Insights from a pristine meteorite. PNAS, 105: 7300-7304.

E-mail: pizzar@asu.edu

\subsection{Billion Year Old Stromatolite Reef of Western Australia: A Rich, Large-Scale Record of Early Biota, Strategies and Habitats}

\section{Abigail Allwood, Mark Anderson \\ California Institute of Technology, Jet Propulsion Laboratory}

The abundant, diverse and relatively well preserved stromatolites of the 3.45 billion year old Strelley Pool Formation, Pilbara Craton, Western Australia, are a potentially rich cache of information about early life and ecosystems. A recent study showed that the stromatolites (laminated sedimentary structures of probable biological origin) formed an isolated peritidal carbonate buildup with attributes resembling a shallow marine microbial reef system (Allwood et al., 2006). However, critical small scale evidence of biological activity, such as microfossils and microbial sedimentary fabrics, has remained elusive due to the destructive effects of chert and carbonate recrystallization. Such evidence is critical to further test the hypothesis that the stromatolites are biogenic; to understand the full range of primitive microbial biosignatures in order to inform the search for life on Mars; and perhaps to gain more detailed insight to the characteristics, capabilities and survival strategies of organisms on the early Earth.

To overcome the pervasive recrystallization that has overprinted sedimentary fabrics in the Strelley Pool Formation stromatolites, we develop a novel method incorporating mesoscale X-ray fluorescence element mapping. A multi scalar sedimentological approach is adopted; integrating such factors as sedimentary fabrics and microfacies, facies assemblages, and depositional architecture of the host deposit. This yields significantly detailed new insights to the way the stromatolites formed. We are able to detect co-variations in recrystallized fabric and stromatolite shape; correlative changes in lamina character and accretionary architecture; changes in stromatolite shape or distribution that coincide with changes in environment; and variations in stromatolite shape or laminar fabric across unchanging environments. Relationships between fabric, sedimentary facies and stromatolite morphologies indicate: that microbes played a role in local mediation of sediment deposition (leading to stromatolite formation); the environmental forces that the microbes were subject to; the likely responsive strategies that microbes adopted; and the resultant effect on stromatolite morphology. As targeted, precise, geochemical and organic geochemical data are obtained in the Strelley Pool Formation, their interpretation is greatly constrained by their relationship with the fabrics and facies they are found in. The approach has proven useful not only in revealing new types of evidence for the origin of the Strelley Pool Formation stromatolites, but also for generating principles that can be applied to other cases. 
Allwood A.C., Walter M.R., Kamber B.S., Marshall C.P., Burch I.W., 2006. Stromatolite reef from the Early Archaean era of Australia. Nature, 44:714-718.

E-mail: Abigail.C.Allwood@jpl.nasa.gov

\title{
Four Oxygen Reductases, Four Evolutionary Histories: Implications for the Emergence of Aerobic Respiration and Early Earth Atmosphere
}

\author{
Celine Brochier-Armanet* ${ }^{1,3}$, Emmanuel Talla ${ }^{2,3}$, Simonetta Gribaldo ${ }^{4}$
}

${ }^{1}$ Université de Provence Aix-Marseille I, France; ${ }^{2}$ Université de la Méditerranée AixMarseille II, France; ${ }^{3}$ Laboratoire de Chimie Bactérienne CNRS UPR9043, Marseille, France; ${ }^{4}$ Unité de Biologie Moléculaire chez les Extremophiles (BMGE), Institut Pasteur, Paris, France

Understanding the origin and evolution of cellular processes is fundamental to understand how biological activity has shaped the history of our planet as well as its biota. We have investigated the distribution of the four types of oxygen reductases - the key enzymes of aerobic respiratory chains, in all available complete archaeal and bacterial genomes, and analyzed their phylogeny. Our results show that each oxygen reductase type has a very different evolutionary history. However, one of them was already present prior to the divergence of Bacteria and Archaea, and was maintained throughout their subsequent diversification. Implications for the emergence of aerobic respiration and early earth atmosphere will be discussed.

\section{Titan: Exploring an Earth-Analogue}

\section{A. Coustenis}

\section{LESIA, Paris-Meudon Observatory, France}

Titan, Saturn's largest satellite was discovered in 1655 by Huygens. Much later, it was found to possess a substantial atmosphere by Kuiper in the 1940s. Titan is today still the only confirmed exobiotic environment known to us. It is also perhaps the most intriguing object in our Solar System. Its uncanny resemblance to our own planet has motivated generations of scientists into studying it from both the space and from the ground, and has initiated the Saturn-bound Cassini/Huygens ESA/NASA mission (which arrived on Titan in July 2004, while the probe descended through the atmosphere and landed on the surface in January 2005). Prior to this extraordinary mission, Titan had been observed from the ground (using large telescopes, such as those in Hawaii and Chile), but also from space (initially with Voyager 1 and 2, with the HST, and recently with ISO). Thus, we know today that the thick atmosphere layer-covering the satellite's mysterious surface-is essentially made of nitrogen, with small amounts of methane and hydrogen. The combination among these mother molecules produces an exciting organic chemistry in Titan's atmosphere, with hydrocarbons and nitriles (one of the latter, $\mathrm{HCN}$, is a prebiotic molecule). These organics are probably produced high up in the ionosphere, as recently discovered by the Cassini/ INMS. As a difference with our own planet we note the absence of significant amounts of oxygen (only traces of $\mathrm{H}_{2} \mathrm{O}, \mathrm{CH}_{4}$ and $\mathrm{CO}_{2}$ have been discovered), as well as the low temperatures prevailing ( $180 \mathrm{~K}$ in the atmosphere and $94 \mathrm{~K}$ on the surface) that delay chemical reactions. The general shape of the thermal profile is, however, quite similar to 
that of the Earth's with temperature inversions predicted at the tropopause and the mesopause.

Titan's surface remained hidden under a veil of a thick aerosol cloud to the visible cameras for a long time, but first from spectroscopy and imaging in the near-IR from the ground we saw that this surface is inhomogeneous, bright on the leading side and darker on the trailing one. Then, with the Cassini orbiter and with the Huygens probe, we uncovered some of the features related with the lower atmosphere and surface of Titan. Thus, we have definite indication today of the presence of significant seasonal and diurnal effects in Titan's atmosphere. In imaging, a large, bright equatorial regionpossibly connected with relief - is found on the leading hemisphere, while bright areas are also observed near the poles. The exact nature of the ground remains to be discovered, but spectroscopy indicates that it is probably a mixture of ices $\left(\mathrm{H}_{2} \mathrm{O}, \mathrm{CH}_{4}\right.$, $\left.\mathrm{CO}_{2} \ldots\right)$, hydrocarbon liquid and rocks.

Our understanding of Titan has been greatly enhanced by the data returned by the CassiniHuygens mission still on location. After this mission, any unanswered questions on the atmosphere, the surface, the interior and the astrobiological aspects of the satellite will forever remain unknown, unless we go back with an optimized orbital tour and advanced instrumentation. Considering the complementary nature of the geological, chemical and evolutionary history of Titan and Enceladus, we are currently studying a new mission to perform in situ exploration of these two objects (Titan/Saturn System Mission), a collaboration between ESA and NASA. Key science measurements and the instrument suites for achieving new science goals have been defined (Coustenis et al., 2008). In particular, we developed conceptual designs for delivering the science payload, including an orbiter, an aerial platform and probes for Titan. The suggested launch date is around or beyond 2018.

I will discuss the implications of recent and future observations on our understanding of Titan.

Coustenis, A., and 154 co-authors, 2008. TandEM: Titan and Enceladus mission. Astrophysical Instruments and Methods, in press.

E-mail: Athena.Coustenis@obspm.fr 


\title{
ORAL PRESENTATIONS
}

\section{Planetary Evolution}

\section{The Organic Chemistry of Nearby Galaxies Measured with a New, Very Broadband Receiver}

G. Narayanan ${ }^{1}$, R. L. Snell ${ }^{1}$, N. A. Erickson ${ }^{1}$, A. Chung ${ }^{1}$, M. Heyer ${ }^{1}$, Y. Min ${ }^{1}$, W. M. Irvine ${ }^{1,2}$

${ }^{1}$ Astronomy Department, University of Massachusetts, Amherst, MA 01003 USA; ${ }^{2}$ The Goddard Center for Astrobiology

Millimeter-wavelength spectra of a number of nearby galaxies have been obtained at the Five College Radio Astronomy Observatory (FCRAO) in Massachusetts using a new, very broadband receiver (Erickson et al., 2007). This instrument, which we call the redshift search receiver (RSR), has an instantaneous bandwidth of $36 \mathrm{GHz}$ and operates from 74 to $110.5 \mathrm{GHz}$, permitting the measurement of most of the $3 \mathrm{~mm}$ spectrum with a single receiver setting. The receiver has been built at UMass/FCRAO to be part of the initial instrumentation for the Large Millimeter Telescope (LMT), a 50-m diameter millimeterwavelength single-dish telescope being built jointly by UMass and the Instituto Nacional de Astrofísica, Óptica y Electrónica in Mexico (Perez-Grovas et al., 2006). The LMT is sited at $4,600 \mathrm{~m}$ elevation at latitude $19^{\circ}$ in the Mexican state of Puebla, permitting good access to the southern sky. It is designed for operation in the $0.85-4 \mathrm{~mm}$ wavelength band.

The new receiver is intended for determination of the redshift and hence distance of distant, dust-obscured galaxies, but it can also be used to investigate the chemistry of galaxies. Since the LMT is not yet complete (we are hoping for initial $3 \mathrm{~mm}$ commissioning this year), the receiver is being tested on the FCRAO $14 \mathrm{~m}$ by measuring the $3 \mathrm{~mm}$ spectra of a number of nearby galaxies. There are interesting differences in the chemistry of these objects, e.g., in the relative strength of emission lines from $\mathrm{HCN}, \mathrm{HNC}, \mathrm{HCO}^{+}, \mathrm{CH}_{3} \mathrm{OH}$, ${ }^{13} \mathrm{CO}, \mathrm{CS}$ and $\mathrm{N}_{2} \mathrm{H}^{+}$(a proxy for $\mathrm{N}_{2}$ ).

Erickson, N. R., Narayanan, G., Goeller, R., \& Grosslein, R. (2007). From Z-Machines to ALMA: (Sub)Millimeter Spectroscopy of Galaxies, 375, 71

Perez-Grovas, A. S., Schloerb, P. F., Hughes, D., \& Yun, M. (2006). SPIE, 6267: 1.

E-mail: irvine@astro.umass.edu

\section{Investigation of Isovaline Enantiomeric Excesses and Other C5 Amino Acids in Carbonaceous Meteorites}

\author{
Jason P. Dworkin, Daniel P. Glavin
}

Goddard Center for Astrobiology, Solar System Exploration Division, NASA Goddard Space Flight Center, Greenbelt, MD 20771, USA

The origin of biological homochirality is one of the most perplexing puzzles to understanding the emergence of life on Earth. While many models have been proposed (e.g. Hazen et al. 2001; Keszthelyi 1984), the only reported non-biologically generated compounds that show a significant enantiomeric excess are a few amino acids in the CM2 Murchison and Murray meteorites (e.g. Pizzarello and Cronin 2000; Pizzarello et al, 2008). 
Of these isovaline ( $\alpha$-ethyl-alanine) is of particular interest since it is typically abundant in CM2 meteorites, is exceedingly rare in biology, and due to its chemical structure is likely to maintain its primordial $\mathrm{D} / \mathrm{L}$ ratio.

Instead of the gas chromatography-mass spectrometry (GC-MS) technique employed by Pizzarello et al., we have used liquid chromatography-fluorescence detection/time of flightmass spectrometry (LC-FD/ToF-MS) to study the enantiomeric ratio of isovaline in the CM2 meteorites Murchison and LEW90500 and the CR2 QUE99177. We have placed particular emphasis on understanding the suite of $\mathrm{C} 5$ amino acids in these meteorites. In doing so, we have determined that $\mathrm{D}$ and $\mathrm{L} 3$-aminopentanoic acid co-elutes with Lisovaline and L-valine under common chromatographic conditions (Glavin and Dworkin 2006) for $o$-phthaldialdehyde/N-acetyl-L-cysteine (OPA/NAC).

We have devised a method to separate these compounds and we will report the actual D/ $\mathrm{L}$ ratios of isovaline in these meteorites and how they compare to the GC-MS measurements of Pizzarello and co-workers.

Glavin, D. P. and Dworkin J. P. (2006) Investigation of isovaline enantiomeric excesses in $\mathrm{CM}$ meteorites using liquid chromatography time of flight mass spectrometry. Astrobiology, 6: 105 .

Hazen, R. M., Filley, T. R. and Goodfriend, G. G. (2001) Selective adsorption of L- and Damino acids on calcite: Implications for biochemical homochirality. Proc. Natl. Acad. Sci. USA, 98: 5487-5490.

Keszthelyi, L. (1984) Review of the origin of asymmetry of biomolecules through weak interaction: Latest developments. Orig. Life Evol. Biosph. 11: 9-21.

Pizzarello S. and Cronin J. R. (2000) Non-racemic amino acids in the Murray and Murchison meteorites. Geochim. et Cosmochim. Acta. 64: 329-338.

Pizzarello S. Huang, Y. and Alexandre M. R. (2008) Molecular asymmetry in extraterrestrial chemistry: Insights from a pristine meteorite.. Proc. Natl. Acad. Sci. USA, 105: 3700-3704.

E-mail: Jason.P.Dworkin@nasa.gov

\title{
Delivery of Exogenous Materials from Comets and Asteroids to the Prebiotic Earth
}

\author{
Jennifer G. Blank
}

Carl Sagan Center for the Study of Life in the Universe, SETI Institute, 515 N. Whisman Rd. Mountain View CA 94043 USA

Comets and asteroids were significant contributors to the inventory of water and organic compounds on the surface of the early Earth and thus may play an important role in the origin of life. Successful delivery requires that some of the organic materials survive the extreme temperatures and pressures associated with impact, and, also, that water accompanies the organic materials. There has been substantial advancement in impact modeling capabilities since the initial work of Chyba and others (1990), which remains widely cited by the Origin of Life community. However, models of chemical reactions under shock are still limited by our lack of relevant empirical and theoretical knowledge in these dynamic and extreme pressure and temperature regimes. Here, I will summarize work that addresses the issue of impact delivery and focus on the phase-state of water during modeled comet-earth and asteroid-earth collisions over a range of impact angles and velocities. On the basis of 
model results (e.g., Liu et al., 2007) generated using a three-dimensional shock physics code (GEODYN), I will infer survivability of organic compounds and liquid water over a range of impact scenarios for comet-Earth and asteroid-Earth collisions. These results will be described in the context of the flux of astromaterials and water (as both liquid and vapor) to the prebiotic Earth.

Chyba, CF, PJ Thomas, L Brookshaw, and C Sagan (1990) Cometary delivery of organic molecules to the early Earth, Science 249: 366-373.

Liu, B. T., I. Lomov, J. G. Blank, and T. H. Antoun (2007) 3-D Simulation of Comet Impact and Survivability of Organic Compounds, Proceedings of the 15 Amer. Phys. Soc. Topical Conference on Shock Compression of Condensed Matter, C304-308.

E-mail: jblank@seti.org 


\section{Prebiotic Syntheses}

\section{Phosphorylation at Convergent Margins}

Nils G. Holm

\section{Department of Geology and Geochemistry, Stockholm University}

Phosphorus is a relatively rare element on Earth but is extremely important for the biological coding of information as well as the transfer of energy and information in living organisms. Phosphorus is scavenged from sea water by ridge-flank hydrothermal activity and is accumulated in oceanic crust. High-energy phosphate compounds are omnipresent in biological systems. Simple pyro- and polyphosphates are used as a form of energy storage in many microorganisms, and it has been proposed that the chemical energy stored in this type of molecules has been used by primitive forms of life on the early Earth. The potential of pyrophosphate formation upon heating of hydrogenated orthophosphates to a few hundred $\mathrm{C}$ in geological environments where the activity of water is low has probably been underestimated. Boron, on the other hand, has never been in focus in biogeochemistry and the study of the global geochemical cycles because it is not a major component of biological macromolecules. Borate is an important component of seawater $(0.4 \mathrm{mmol} / \mathrm{kg})$ and one of the components that determines the alkalinity of marine environments. Like phosphorus it is scavenged from seawater by cooling rocks of oceanic crust and upper mantle and is released again upon heating at convergent margins, at which abiotic formation of aldehydes also occurs. Boron has a strong affinity for organic material since it forms trigonal and tetrahedral complexes with oxygen groups. For instance, boric acid and borate readily form complexes with a wide variety of sugars and other compounds containing cis-hydroxyl groups, like humic substances. Accordingly, pentoses such as ribose are known to form stable borate complexes. The binding preferences of borate to pentoses has been determined to be ribose $>$ lyxose $>$ arabinose $>$ xylose $(\mathrm{Li}, 2005)$. The ribose molecule may be stabilized by borate that binds to the $2^{\prime}$ and $3^{\prime}$ positions of the furanose form of ribose. The fact that ribose is stabilized by borate may change our opinion of the formose reaction as a seemingly random and nonselective reaction into a very precise geochemical preRNA process. The formose reaction was, for a while, an outdated concept for abiotic synthesis of carbohydrates. However, because of borate complex formation it is still possible that it is responsible for prebiotic formation of ribose in natural environments and that this may occur in close vicinity to abiotic purine synthesis and phosphorylation processes in alkaline hydrothermal environments of convergent margins. Once pyrophosphate is available, phosphorylation of ribose and/or nucleosides may occur.

Li, Q., Ricardo, A., Benner, S.A., Winefordner, J.D., and Powell, D.H. (2005). Desorption/ ionization on porous silicon mass spectrometry studies on pentose-borate complexes. Analytical Chemistry 77, 4503-4508.

E-mail: nils.holm@geo.su.se 


\title{
Models of Abiotic Synthesis of Adenosine Mono-, Di- and Triphosphate
}

\author{
Taisiya A. Telegina, Michael P. Kolesnikov, Mikhail S. Kritsky
}

\section{A.N. Bach Institute of Biochemistry, Russian Academy of Sciences, Moscow, Russia}

The first step of ATP synthesis, i.e. the de novo formation of 5'-AMP molecule, is achieved in organisms via a multistage enzymatic process in which adenine heterocycle is built up on the ribose-5-phosphate pedestal from $\mathrm{C}$ and $\mathrm{N}$ atoms originating from formic acid, carbon dioxide, glutamine, glycine and aspartic acid. We showed that under abiotic conditions 5'-AMP can be formed from the same precursors, i.e. the mixture of ribose, potassium phosphate, sodium bicarbonate, ammonium formate, glutamine, glycine and aspartic acid. After $40 \mathrm{~min}$ incubation of anhydrous mixture of these compounds at $85^{\circ} \mathrm{C}$ in oxygen-free atmosphere, $5^{\prime}$-AMP was identified among reaction products by using HPLC for isolation of this nucleotide. The phosphorylation of this nucleotide product gave rise to ATP which was detected by a highly specific luciferin-luciferase luminescence test. The yield of 5'-AMP (calculated to initial ribose content) was about $3-5 \%$. The efficiency of the same set of chemical precursors for abiotic and biological synthesis of 5'-AMP is of interest in context of the development of metabolic pathway of purine nucleotides biosynthesis in early stages of evolution.

According to results of laboratory modeling, in prebiotic world there existed various options for photon energy conservation in energy rich phosphoanhydride bonds of ADP and ATP including the photophosphorylation processes, which did not need any organic sensitizers. For instance, the UVA irradiation of ADP molecules adsorbed on montmorillonite particles led to their phosphorylation by orthophosphate and formation of ATP with the yield of ca. $30-40 \%$. In the case of 5'-AMP photophosphorylation, the yield of ATP was ten times lower (Kritsky, Kolesnikov and Telegina, 2007).

In the other model, the photophosphorylation process was sensitized by abiogenic flavin pigment present in flavoproteinoid microspheres formed after a self-assembly in aqueous medium of the products of thermal condensation of a mixture of glycine, glutamic acid and lysine. The process was induced both by the UVA- and visible (blue) radiation. The yield of ATP after ADP phosphorylation was ca. $20-30 \%$ and the yield of ATP formed as a result of 5'-AMP photophosphorylation was about 10\% (Kritsky, Kolesnikov and Telegina, 2007; Kolesnikov, Telegina, Lyudnikova, and Kritsky, 2008). The photophosphorylation system was active under oxygenic conditions. In the deaerated medium it showed a full activity in case $\mathrm{H}_{2} \mathrm{O}_{2}$ or an alternative, nonoxygenic electron acceptor such as $\mathrm{Fe}^{3+}$-cytochrome $c$ were present. The phosphorylation mechanism has no analogs in organisms. It likely involves a direct interaction of semiquinone flavin molecule with ADP, the formation of ADP radical and its phosphorylation by orthophosphate.

Supported by Presidium of Russian Academy of Sciences, Program of Basic Research No 18 and by grants from Russian Foundation for Basic Research No 07-04-00460-a and No 06-04-90599 BNTS_a.

Kritsky, M.S., Kolesnikov, M.P., and Telegina, T.A., (2007) Modeling of abiogenic synthesis of ATP. Doklady Biochemistry and Biophysics, 417:313-315.

Kolesnikov, M.P., Telegina, T.A., Lyudnikova, T.A., and Kritsky, M.S., (2008) Abiogenic photophosphorylation of ADP to ATP sensitized by flavoproteinoid microspheres. Origins of Life and Evolution of Biosphere, 38(3): 243-255.

E-mail: telegina@inbi.ras.ru 


\title{
Oxaloacetate-to-Malate Conversion by Mineral Photoelectrochemistry: Implications for the Viability of the Reductive Tricarboxylic Acid Cycle in Prebiotic Chemistry
}

\author{
Marcelo I. Guzman, Scot T. Martin
}

School of Engineering and Applied Sciences and Department of Earth and Planetary Sciences, Harvard University, Cambridge, Massachusetts, U.S.A. 02138

There are five known mechanisms by which autotrophic organisms fix carbon (Thauer, 2007). Of these, however, a reductive tricarboxylic acid (rTCA) cycle, has been proposed as the most plausible metabolic pathway of $\mathrm{CO}_{2}$ fixation at the time life originated (Wachtershauser, 1990). Moreover, the carboxylic acids produced by the rTCA cycle are possibly a biosynthetic core of initial life (Smith and Morowitz, 2004). Recently, some of the endoergic reductive steps of the rTCA cycle were demonstrated as feasible through mineral photoelectrochemistry by the semiconductor mineral ZnS (Zhang and Martin, 2006). In this context, the reductive conversion kinetics of oxaloacetate (OAA) to malate (MA) by $\mathrm{ZnS}$ mineral photoelectrochemistry were studied from 5 to $50^{\circ} \mathrm{C}$ at $\mathrm{pH}=7.0$ and from 1 to $10 \mathrm{mM}$ OAA for wavelengths of $200 \mathrm{~nm}$ and longer. The quantum efficiency $\Phi_{\mathrm{MA}}$ of MA production from the photoelectrochemical reduction of OAA followed $\Phi_{\mathrm{MA}}=0.13$ [OAA] $\left(2.1 \times 10^{-3}+[\mathrm{OAA}]\right)^{-1}$ and was independent of temperature. To evaluate the importance of this forward rate under a prebiotic scenario, we also studied the temperature-dependent rate of the backward thermal decarboxylation of oxaloacetate to pyruvate (PA), which followed an Arrhenius behavior as $\log \left(k_{-2} / \mathrm{s}^{-1}\right)=11.74-4,956 / T$. These measured rates were employed in conjunction with the indirectly estimated carboxylation rate of pyruvate to oxaloacetate to assess the possible importance of mineral photoelectrochemistry in the conversion of oxaloacetate to malate under several scenarios of prebiotic conditions on early Earth. As an example, our analysis shows that there is $90 \%$ efficiency and 3-year/cycle forward velocity for the OAA $\rightarrow$ MA step of the rTCA cycle at $280 \mathrm{~K}$. Efficiency and velocity both decrease for increasing temperature. These results suggest high viability for mineral photoelectrochemistry as an enzyme-free engine to drive the rTCA cycle through the early eons of early Earth, at least for the investigated OAA $\rightarrow$ MA step.

Smith, E. and Morowitz, H. J. (2004). Universality in intermediary metabolism. PNAS, 101:13168-13173.

Thauer, R. K. (2007). A Fifth Pathway of Carbon Fixation. Science, 318, 1732-1733. Wachtershauser, G. (1990). Evolution of the first metabolic cycles. PNAS, 87, 200-204. Zhang, X. V. and Martin, S. T. (2006). Driving Parts of Krebs Cycle in Reverse through Mineral Photochemistry. J. Am. Chem. Soc., 128, 16032-16033.

E-mail: mig@deas.harvard.edu

\section{Irradiation of Nucleic Acid Bases Adsorbed in Na-Montmorillonite in the Context of Chemical Evolution}

Betzabe Zamora, Adriana Melndez, Andres Guzman, Alicia Negrn-Mendoza, Sergio Ramos-Bernal

Instituto de Ciencias Nucleares, Universidad Nacional Autnoma de Mexico, UNAM. Cd. Universitaria, A.P. 70-543, 04510 Mexico, D.F. Mexico

Nucleic acid bases are part of important compounds in biological systems, such as genetic and energy utilization processes. Most of the bases are readily formed in prebiotic conditions. Their 
synthesis and stability in environmental conditions is of paramount importance in chemical evolution (Miller and Orgel, 1974). On the other hand, Clay minerals might have played an important role on the early Earth. They are considered the most likely inorganic material to promote organic reactions at the interface of the hydrosphere and lithosphere (Bernal, 1951). The relevance of clay minerals in the emergency of the origin of life is due to their ancient origin, wide distribution and especially for their physico-chemical properties (Negron-Mendoza and Ramos-Bernal, 2004). There are several routes for the synthesis of nucleic acid bases in simulation experiments of the primitive Earth (Miller and Orgel, 1974). Studies about the stability in primordial environments are scare, specially related to the study of the stability of nucleic acid bases in an aqueous medium, at high temperatures, or in the presence of high radiation fields In this work, we study the adsorption, co-adsorption phenomena and protection role of clay in nucleic acid bases. To this end, we determine the survival of bases exposed to a high radiation field in aqueous solution and adsorbed in a clay mineral. The results showed the protection role of the clays toward ionizing radiation. Bases are able to resist radiation, while they are adsorbed in a clay mineral. This is a distinct advantage since the molecules that were formed by ultraviolet light, ionizing radiation, or electric discharges had to survive in order to interact with each other to form more complex molecules.

This work was partially supported by PAPIT grant IN223406-3.

Bernal, J.B. (1951). The Physical Basis of Life. Routledge and Kegan Paul, London. Miller, S.L. and Orgel, L. (1974). The Origins of Life on Earth. Prentice-Hall, Inc., New Jersey. Negron-Mendoza, A. and Ramos-Bernal, S (2004). The role of clays in the origin of life. In Seckbach, J., editor, Origins: Genesis, evolution and diversity of life, pages 183-194. Kluwer Academic Publisher, Netherlands.

E-mail: negron@nucleares.unam.mx

\section{In Silico Prebiotic Chemistry: Aluminosilicate Surfaces As Promoters for the Peptide Bond Formation}

Piero Ugliengo ${ }^{1}$, Albert Rimola ${ }^{2}$, Mariona Sodupe ${ }^{2}$

${ }^{1}$ Dipartimento di Chimica I.F.M, NIS Centre of Excellence and INSTM National Consortium, Università degli Studi di Torino, via P. Giuria 7-10125 Torino, Italy; ${ }^{2}$ Departament de Química, Universitat Autònoma de Barcelona, Bellaterra 08193, Spain

The route for which basic molecular building blocks such as amino acids and nucleobases were joined in a proper and controlled way in order to make the first active biopolymers during primitive Earth is an intriguing question that nowadays still remains open in the area of the prebiotic chemistry. Indeed, even for the condensation of glycine (the simplest amino acid) the reaction occurring in highly diluted water solution is thermodynamically disfavoured. An early suggestion form Bernal in 1951 (Bernal, 1951) advocated the special role of mineral clays as promoters for the condensation of monomer building blocks since they provide adsorption sites that, on one hand, may immobilize, concentrate and protect amino acids and peptides from hydration and, on the other hand, may induce a lowering of the activation barrier because of the presence at the surface of catalytic active sites. Along this line, Orgel (Orgel, 1998) stated that successive cycles of condensation occurring on mineral surfaces causes elongation of the synthesized peptide which remains almost irreversibly adsorbed, so that its destructive hydrolysis, will become more and more improbable. In the present contribution, a detailed theoretical mechanistic study addressed to the peptide bond formation catalyzed by an aluminosilicates surface is presented. A large and 
realistic cluster model cut out from the sanidine feldspar surface rich in both Lewis and Brønsted sites has been adopted as a catalytic surface. The free energy profiles for the condensation of glycine molecules on the sanidine surface yielding glycylglycine (Figure 1) and glycylglycylglycine as reaction products have been simulated using the ONIOM2 [B3LYP/6-31 + G(d,p):MNDO] level of theory. Results indicate that the catalytic interplay between Lewis and Brønsted sites is a key factor to favour the reactions (Rimola, et al. 2007). Additionally, theoretical results show that purely London forces between the biomolecules and the surface play a crucial role in the condensation processes because they greatly stabilize the peptide at the surface, as suggested by Orgel (Orgel, 1998). Finally, further discussion concerning the controversy between peptide polymerization vs peptide hydrolysis is also addressed by the explicit introduction of water molecules in the reaction process.
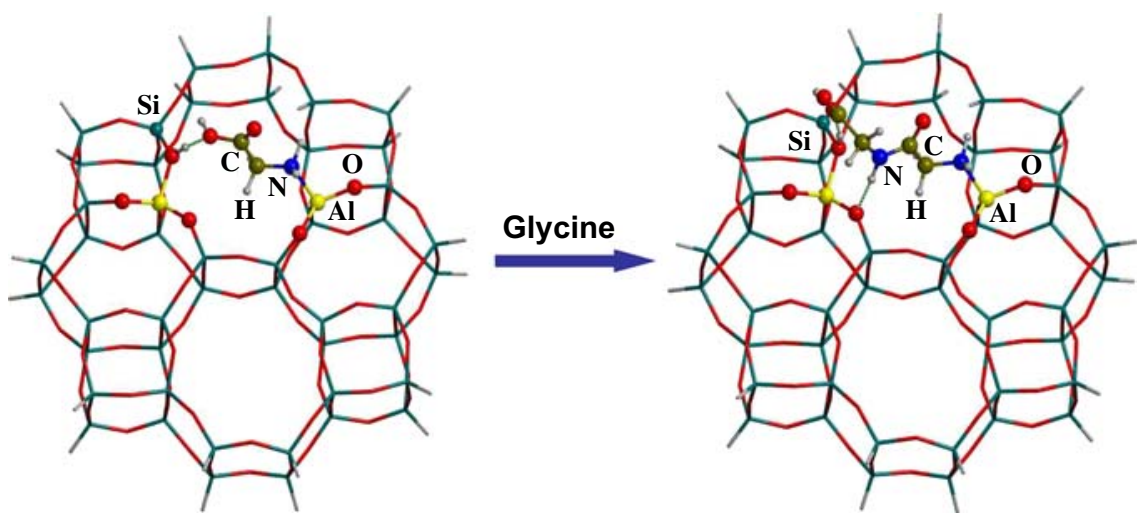

Bernal, J. D. (1951). The Physical Basis of Life. Routledge and Kegan Paul, London.

Orgel, L. E. (1998). Polymerization on the rocks: Theoretical introduction. Orig. Life Evol. Biosph., 28: 227-234.

Rimola, A., Sodupe, M., and Ugliengo, P. (2007). Aluminosilicate surfaces as promoters for peptide bond formation: An assessment of Bernal's hypothesis by ab initio methods. J. Am. Chem. Soc., 129: 8333-8344.

E-mail: piero.ugliengo@unito.it 


\section{Origins of Genetic Information}

\section{Seeking Robustness: High Neutrality and Stable Structures in Populations of RNA Sequences}

Javier M. Buldú ${ }^{1}$, Jacobo Aguirre ${ }^{2}$, Susanna C. Manrubia

${ }^{1}$ Grupo de Dinámica no Lineal y Teoría del Caos. Dept. of Physics, Universidad Rey Juan Carlos, c/ Tulipán s/n, 28933 Móstoles, Madrid, SPAIN; ${ }^{2}$ Centro de Astrobiología, INTACSIC. Ctra. de Ajalvir km. 4, 28850 Torrejón de Ardoz, Madrid, SPAIN

High replication error rates strongly limit the length of sequences that can transmit reliable information. However, this restriction is alleviated when considering that selection acts on the phenotype: the extremely large degeneracy between genotype and phenotype spaces confers robustness (in the form of increased molecular neutrality) to RNA populations. Sets of sequences folding into the same secondary structure form neutral networks in the genome space: A population of sequences can move on such networks without seeing its functionality affected, as far as the secondary structure is concerned. The adjacency matrix $A_{i j}$ states whether sequence $i$ can be accessed (through a single point mutation) from sequence $j$, thus fully describing the structure of the neutral network. In this work we study two properties of such networks that affect robustness: its areas of maximal neutrality against mutations and the minimum free energy associated to the folded state of each sequence. The topological properties of neutral networks determine (a) the time $T_{n}$ required to attain maximally neutral states and (b) the diversity of sequences in the population at that state. When information on the energy associated to each sequence is included, topology also affects (c) survivability of the populations under temperature fluctuations. The position of the maximally neutral region and the diversity of the population once that region has been attained are analytically obtained through the principal eigenvalue and the corresponding eigenvector of $A_{i j}$. The relaxation time to that state is obtained from non-principal eigenvalues of $A_{i j}$. Finally, if each sequence has a minimum free energy associated, temperature increases destabilize subsets of sequences (not necessarily connected in the neutral network) and push the population towards regions of low energy. Reaching a compromise between attaining high molecular neutrality and being stable against temperature changes could have been a crucial step in the survivability of early populations of replicating RNA molecules.

Buldú, J. M., Aguirre, J., and Manrubia, S. C. Seeking robustness: high neutrality and stable structures in populations of RNA sequences. In preparation.

Schuster, P. (2006). Prediction of RNA secondary structures: from theory to models and real molecules. Rep. Prog. Phys. 69:1419-1477.

van Nimwegen, E., Crutchfield, J. P., and Huynen, M. (1999). Neutral evolution of mutational robustness. Proc. Natl. Acad. Sci. USA 96: 9716-9720.

E-mail: cuevasms@inta.es 


\section{Water: From the Nonenzymatic Phosphorylation of Nucleosides to the Nonenzymatic Ligation of Oligonucleotides}

Giovanna Costanzo ${ }^{1}$, Fabiana Ciciriello ${ }^{2}$, Samanta Pino $^{2}$, Diego Pesce $^{2}$, Michele Graciotti ${ }^{2}$, Ernesto Di Mauro ${ }^{2}$

${ }^{1}$ IBPM, CNR, Rome, Italy; ${ }^{2}$ Dipartimento di Genetica e Biologia Molecolare, Università di Roma "Sapienza", Italy

In trying to reconstruct the origin of informational polymers we have followed the path of simplicity. All the relevant steps can occur abiotically and non-fastidiously. Nucleosides can be phosphorylated in water from simple phosphate donors. 2'AMP, 3'AMP, 5'AMP, 2',3'cAMP and 3',5'-cAMP are formed. 2',3'-cAMP and 3',5'-cAMP can form oligomers in water, at moderate temperature and without the help of catalysts or of additional activation. 2'AMP, 3'AMP and 5'AMP do not. Adenine-based oligomers undergo spontaneous terminal ligation in water, affording dimers and tetramers. The only limiting constraint is $\mathrm{pH}$. The possibility that this reaction is the starting mechanism from which replication of genetic polymers evolved will be discussed.

E-mail: ernesto.dimauro@uniroma1.it

\section{RNA Synthesis by Mineral Catalysis}

Michael F. Aldersley ${ }^{1}$, Prakash Joshi ${ }^{1}$, John Delano ${ }^{2}$, James P. Ferris ${ }^{1}$

${ }^{1}$ Rensselaer Polytechnic Institute, Troy NY 12180 USA; ${ }^{2}$ University at Albany, Albany, NY, 12222 USA

The RNA World hypothesis proposes that RNA was the most important biopolymer in the primitive life on the Earth. It served as a catalyst as well as a repository of genetic information. We discovered that 40-50 mers of RNAs are formed by the montmorillonite clay catalysis of the reaction of activated monomers. The montmorillonite not only catalyzes the formation of long oligomers but its selectivity in the synthesis controls the sequences of RNAs and also the regioselectivity of the phosphodiester bonds formed $\left(2^{\prime}, 5^{\prime}\right.$ or $\left.3^{\prime}, 5^{\prime}\right)$.

As we explore the mechanism of montmorillonite catalysis and the properties of the RNA oligomers formed, we find that not all montmorillonites are catalysts. Those having a lower layer charge allow the activated monomers to intercalate the montmorillonite platelets where catalysis occurs. Those with a higher layer charge have a greater concentration of cations in the interlayer preventing monomers from intercalating between the montmorillonite platelets. The montmorillonites that are catalysts all have similar elemental compositions.

We are currently investigating if the RNA oligomers formed by montmorillonite are catalysts. Oligomers of RNA are prepared from mixtures of 2, 3 or 4 activated RNA monomers. They are then passed through an affinity column in which an agarose gel has an attached spacer arm with the target molecule (amino acids, nucleotides etc.) attached to its end. Those RNA oligomers that bind to the target molecule will be isolated and tested for their ability to catalyze reactions of the target molecule. If catalysis is observed this finding will be consistent with the RNA world hypothesis that these RNAs are catalysts.

E-mail: ferrij@rpi.edu 


\title{
Not to Put the Cart Before the Horse
}

\author{
A. G. Cairns-Smith
}

\section{Chemistry Department, Glasgow University, UK}

Darwin fully acknowledged the difficulties in seeing how such a thing as an eye might have evolved through natural selection (Darwin 1859, Chapter 6), but he knew of many lesser examples that could clearly have arisen that way. If the detailed, well adapted, shape of a bird's beak could have arisen through natural selection without the need for a prior creator, then Nature can indeed act as if it were an engineer, producing what seem to be purposebuilt structures, and giving an impression of foresight. But, really, no mysterious view of the future is required. What is absolutely required for nature's engineer to get to work is remarkable all the same: it is a kind of memory of what succeeded in the past. So this is the question that should be the first focus of our attention: What are the simplest genetic memories that we can imagine working in a primitive geochemical milieu? The RNA world idea has been a great inspiration, but this system is already too sophisticated and too far from ordinary geochemistry to be a likely beginner in the evolution game. I have suggested that the mineral world provides us with several candidates for more primitive genetic materials (Cairns-Smith 2005, 2008 and references therein). I will argue against the usual approach to the puzzle of the origin of life, which looks for ways in which the present molecules of life might have arisen as a prelude to a Darwinian evolution. I think that this puts the cart before the horse. I will suggest rather that natural selection came first, based on inorganic materials, and that "the molecules of life" were a crucial but later part of the story.

Cairns-Smith, A. Graham (2005). Sketches for a mineral genetic material. Elements, 1: 157-161.

Cairns-Smith, A. Graham (2008). Chemistry and the missing era of evolution. Chemistry: A European Journal, 14: 3830-3839.

Darwin, C. (1859) The Origin of Species. John Murray, London (reprinted by Penguin Books).

E-mail: grahamcs@chem.gla.ac.uk

\section{The Evolving RNA Machine for Protein Biosynthesis}

\author{
Ilana Agmon, Chen Davidovich, Anat Bashan, Ada Yonath
}

Dept of Structural Biology, Weizmann Institute, Rehovot 76100, Israel

The ribosome's active site, the peptidyl transferase center (PTC), resides within a highly conserved region of the large ribosomal subunit, comprised of 180 nucleotides arranged as a pseudo symmetrical two-fold region in all known structures, confining a void that provides the space required for the motions involved in the translocation of the incoming ribosome substrates, namely the aminoacylated-tRNA molecule. Furthermore, the elaborate architecture is capable of positioning both ribosome substrates, namely the aminoacylated and the peptidyl tRNAs molecules, in stereochemistry required for peptide bond formation and for substrate-mediated catalysis, as well as for the successive reactions, hence enabling amino acid polymerization. Consistent with comprehensive mutagenesis experiments as well as with quantum mechanical calculations, the nucleotides positioned at "walls" of this 
region appear to navigate this motion and their interactions with the translocating aminoacylated tRNA seem to stabilize the transition state of peptide bond formation.

The overall fold of the RNA backbone of this region resembles motifs identified in "ancient" and "modern" RNA molecules of comparable size, regardless of their sequences. Similarly, the symmetry of this region relates the backbone fold and nucleotides orientation, but not nucleotide sequence, hence emphasizing the superiority of functional requirement over sequence conservation.

The extremely high conservation of this region throughout all known kingdoms of life, the universality of its three dimensional structure, its central location within the ribosome, and the inherent tendency of RNA segments of comparable size to dimerize, support the hypothesis that the ancient ribosome evolved by gene duplication or gene fusion.

Preliminary experimental results and conceptual issues will be presented and discussed.

E-mail: ada.yonath@weizmann.ac.il

\section{Chemical Evolution of Peptides}

Bernd M. Rode, Daniel Fitz, Thomas Jakschitz

Theoretical Chemistry Division, Institute of General, Inorganic and Theoretical Chemistry, University of Innsbruck, Austria

The Salt-Induced Peptide Formation (SIPF) reaction is discussed as the simplest and most plausible way for the formation of peptides under primordial earth conditions. Starting from amino acids in the presence of sodium chloride and copper chloride, peptides are formed in aqueous solution within a few days of evaporation/re-dissolution cycles mimicking day/ night cycles.

The SIPF reaction has numerous properties suggesting their relevance in prebiotic chemistry leading to the origin of life. It prefers the biologically relevant alpha amino acids over their beta and gamma analogues, it works with all amino acids investigated so far and under varying ambient conditions. Further, it can be conducted in the presence of clay minerals, which stabilise the peptides against subsequent hydrolysis and favour the formation of longer chains.

Instead of arbitrary amino acid sequences, the SIPF reaction preferentially produces specific sequences, whose probabilities can be measured by the yields obtained. A comparison of these preferred sequences with the sequences found in the membrane proteins of archaea and procaryonta yields a strong coincidence, further underlining the relevance of this reaction for chemical evolution.

The SIPF reaction also provides an explanation for the biohomochirality using L amino acids, which will be presented in a separate contribution.

E-mail: Bernd.M.Rode@uibk.ac.at

\section{Oligopeptide Formation Under Hydrothermal Conditions Using a Micro-flow Hydrothermal Reactor}

Kunio Kawamura, Hitoshi Takeya, Ai Akiyoshi, Masanori Shimahashi

Department of Applied chemistry, Graduate School of Engineering, Osaka Prefecture University

Phylogenic analyses of the last common ancestor (LCA) of currently existing organisms have suggested that life originated in hydrothermal environments on primitive earth while the nature 
of LCA remains still disputed (Holm, 1992; Miller and Lazcano, 1995). Successful simulation experiments conducted under hydrothermal vent conditions support this hypothesis. However, the length and yield of the oligopeptide-like molecules formed in these experiments seemed insufficient for the preservation of biochemical functions (Imai et al., 1999). Diketopiperazines (DKPs) formation from dipeptides is a stumbling block for the prebiotic formation of oligopeptides. We have established a hydrothermal micro-flow reactor system (HFR), which enables monitoring hydrothermal reactions within $0.002-180 \mathrm{~s}$ at temperatures up to $400^{\circ} \mathrm{C}$ (Kawamura, 2000). By using HFR, we have discovered possible pathways for the oligopeptide formation (Kawamura et al., 2005; Kawamura and Shimahashi, 2008). Here we show details and further investigations concerning these reactions. First, during the degradation of L-alanyl-L-alanyl-L-alanyl-L-alanine ((Ala)4) under hydrothermal conditions, (Ala) 5 was detected. This was due to the elongation of (Ala) 4 with alanine monomer, which was formed by partial degradation of (Ala)4. The elongation reaction proceeds at $250-330^{\circ} \mathrm{C}$ at $\mathrm{pH} 2-12$; the elongation was 10-100 times more efficient and much faster than the previous oligopeptide formation under the simulated hydrothermal condition (Imai et al., 1999). However, (Ala)2 and (Ala)3 were quickly converted to DKPs. Second, the formation of oligopeptide-like molecules of length up to 20-mers proceeded from L-glutamic acid (Glu) and L-aspartic acid (Asp). Yields of up to $0.17-0.57 \%$ were obtained in an acidic solution within $13-183 \mathrm{~s}$ at $250-310^{\circ} \mathrm{C}$, as evaluated by matrix-assisted laser desorption/ionization mass spectrometry analysis and high-performance liquid chromatography analyses. The oligopeptide-like molecules were assigned as pyroglutamic acid-capped Asp oligopeptides with linear and/or branched linkages. During the elongations, DKP isomers were not detected. These findings imply that higher oligopeptides could have effectively formed under hydrothermal conditions if some additives, such as mineral catalysts, accelerate the oligopeptide formation or inhibit the formation of DKP isomers.

Holm, N. G. editor (1992), Special issue. Origins Life Evol. Biosphere, 22:1-242.

Imai, E., Honda, H., Hatori, K., Brack, A., and Matsuno, K. (1999). Elongation of oligopeptides in a simulated submarine hydrothermal system. Science, 283:831-833.

Kawamura, K. (2000). Monitoring hydrothermal reactions on the millisecond time scale using a micro-tube flow reactor and kinetics of ATP hydrolysis for the RNA world hypothesis. Bull. Chem. Soc. Jpn., 73:1805-1811.

Kawamura, K. and Shimahashi, M. (on line first). One-step formation of oligopeptide-like molecules from Glu and Asp in hydrothermal environments. Naturwissenschaften.

Kawamura, K., Nishi, T., and Sakiyama, T. (2005). Consecutive elongation of alanine oligopeptides at the second time range under hydrothermal conditions using a micro flow reactor system. J. Am. Chem. Soc., 127:522-523.

Miller, S. L. and Lazcano, A. (1995). The origin of life - did it occur at high temperatures? J. Mol. Evol., 41:689-692.

E-mail: kawamura@chem.osakafu-u.ac.jp

\section{Early History of the Translation Machinery}

\section{George Fox}

Dept. Biology and Biochemistry, University of Houston, Houston, Texas

The translation machinery has been extensively refined and improved over the course of evolutionary history. Evidence for its ancient origins exists in that the majority of the most 
universal genes that were likely present in the last common ancestral populations are involved in translation. Ongoing efforts are focused on identifying which ribosomal proteins originated in the ribosome and which were recruited to it in later times. Although many ribosomal proteins are universally distributed, it is unlikely that even these are equally old. Utilizing information from ribosomal assembly maps, functional roles, ribosomal and genomic locations a proposal is made regarding the relative age of these most conserved proteins. In particular, it is argued that the oldest ribosomal proteins are likely L2, L3 and L4. Other ribosomal proteins may have been derived from these.

E-mail: fox@uh.edu 


\title{
Origins of Homochirality
}

\author{
Enantiomeric Enrichment on the Prebiotic Earth. A Working Laboratory Model, \\ Providing Spontaneous and Continuous Amplifications of Small Enantiomeric \\ Excesses
}

Stanley I. Goldberg

Department of Chemistry, University of New Orleans, New Orleans, Louisiana 70119, USA

A rectangular glass tank, containing water and sand arranged to represent a large lake or sea surrounded by gently sloping beaches, was built to model the enantiomeric enrichment process suggested earlier [S. I. Goldberg (2007), Orig. Life Evol. Biosph., 31, 55-60].

The "sea" is a dilute aqueous solution of a chiral, nonracemic compound with initially low $(10 \%)$ enantiomeric excess, which, through the action of evaporative pumping [K. J. Hsu and C. Siegenthaler (1969), Sedimentology, 12, 11-25], is brought to the surface of the beach by the energy supplied by a heat lamp (the sun) and evaporated-providing crystals enriched in the more abundant enantiomer, (Goldberg, 2007). These are washed down the sloping beach into the "sea" by an aqueous spray (rain). In this way, the enantiomeric purity of the compound in the "sea" was slowly but continually raised from $10 \%$ to $36 \%$ e.e. (so far) after 19 weeks of operation.

E-mail: sgoldber@uno.edu

\section{Amino Acids and the Asymmetric Origin of Life}

Uwe J. Meierhenrich ${ }^{1}$, Jean-Jacques Filippi ${ }^{1}$, Katharina Breme ${ }^{1}$, Rodolphe Perriot $^{1}$, Laurent Nahon ${ }^{2}$, Jan Hendrik Bredehöft ${ }^{3}$, Jun-ichi Takahashi ${ }^{4}$, Wolfram H.-P. Thiemann ${ }^{5}$, Soeren V. Hoffmann ${ }^{6}$

${ }^{1}$ University of Nice-Sophia Antipolis, CNRS UMR 6001, avenue Valrose, 06108 Nice, France; ${ }^{2}$ Synchrotron SOLEIL, l'Orme des Merisiers, St Aubin, BP48, 91192 Gif sur Yvette, France; ${ }^{3}$ Open University, PO Box 197, Milton Keynes, MK7 6BJ, United Kingdom; ${ }^{4}$ NTT Microsystem Integration Laboratories, 3-1, Morinosato Wakamiya, Atsugi 243-0198, Japan; ${ }^{5}$ University of Bremen, Dept. of Physical Chemistry, Leobener Straße, 28359 Bremen, Germany; ${ }^{6}$ University of Aarhus, Institute for Storage Ring Facilities, Ny Munkegade, 8000 Aarhus C, Denmark

Amino acids, the molecular building blocks of proteins (enzymes), certainly played a key role in both the emergence of life on Earth and the development of biomolecular asymmetry, i.e. homochirality. We experimentally simulated the abiotic formation of amino acids and diamino acids in interstellar ices by the effect of UV irradiation on $\mathrm{CO}, \mathrm{CO}_{2}, \mathrm{CH}_{3} \mathrm{OH}, \mathrm{NH}_{3}$, as well as $\mathrm{H}_{2} \mathrm{O}$ and identified 16 amino acids among the remaining products (Muñoz Caro et al. 2002; Meierhenrich, 2008). The presence of diamino acids in the Murchison meteorite verified the above simulation experiment (Meierhenrich et al. 2004). The identified amino acids were racemic, since the experiment was performed under symmetric conditions: the photoreaction was performed with unpolarized light, directed magnetic fields were not applied, an achiral crystal was used as 
support etc. However, interstellar electromagnetic radiation is asymmetric, namely circularly polarized. Here we report on enantioselective photolysis of chiral amino acids under interstellar conditions. First, circular dichroism CD spectra of amino acids including $\alpha$-methyl amino acids such as isovaline were recorded in the amorphous solid state. For the first time, CD spectra in the vacuum UV spectral region were obtained where the photon energy is higher than the dissociation energy of the amino acids allowing enantioselective photolysis reactions. Second, in order to achieve vacuum UV asymmetric photodecomposition of racemic mixtures of solid state amino acids, circularly polarized synchrotron radiation was used to irradiate the samples. After photodecomposition, the enantiomeric excess was found to be $+2.6 \%$ in the case of leucine (Meierhenrich et al. 2005), data on other amino acids will be presented. The results will be verified by the 'chirality-experiment' onboard the Rosetta Lander, which will allow the quantification of chiral organic molecules on a cometary surface (Thiemann and Meierhenrich, 2001).

Meierhenrich, U. J. (2008). Amino acids and the asymmetry of life - caught in the act of formation. Springer, Berlin, Heidelberg, New York.

Meierhenrich, U. J., Muñoz Caro, G. M., Bredehöft, J. H., Jessberger, E. K., Thiemann, W. H.-P. (2004). Identification of diamino acids in the Murchison meteorite. Proc. Natl. Acad. Science, 101:9182-9186.

Meierhenrich, U. J., Nahon, L., Alcaraz, C., Bredehöft, J. H., Hoffmann, S. V., Barbier, B., Brack, A. (2005). Asymmetric vacuum UV photolysis of the amino acid leucine in the solid state. Angew. Chem. Int. Ed., 44:5630-5634.

Muñoz Caro, G. M., Meierhenrich, U. J., Schutte, W. A., Barbier, B., Arcones Segovia, A., Rosenbauer, H., Thiemann, W. H.-P., Brack, A., Greenberg, J. M. (2002). Amino acids from ultraviolet irradiation of interstellar ice analogues. Nature, 416:403-406.

Thiemann, W. H.-P., Meierhenrich, U. J. (2001) ESA mission ROSETTA will probe for chirality of cometary amino acids. Orig. Life Evol. Biosphere 31:199-210.

E-mail: Uwe.Meierhenrich@unice.fr 


\title{
RNA World
}

\section{Evolution of RNA Cooperation on the Rocks}

\author{
Sergio Branciamore ${ }^{1,2}$, Walter de Back $^{2}$, Enzo Gallori ${ }^{1}$
}

${ }^{1}$ Department of Animal Biology and Genetics, University of Florence, Via Romana 17/19, 50125 Firenze; ${ }^{2}$ Collegium Budapest. Institute for Advanced Study. Szentháromság utca 2. H-1014 Budapest, Hungary

The appearance of cooperative interaction between self-replicating molecules constitutes the first major transition in these replicators evolution towards the earliest forms of life (Maynard-Smith and Szathmary 1995). Presumably, these replicators interacted through a common metabolic pathway, in which all performed a specific enzymatic function. This implies that, at some point in the RNA world (Gilbert, 1986; Joyce and Orgel, 1999), two or more molecular species with specific and complementary catalytic activities must have been found, in the same place and at the same time, that enabled a stable metabolic pathway. Given the enormous sequence space, plus the fact that there is no selective reason for fixation of a particular ribozyme without a pre-existent pathway, it seems almost impossible that a functional metabolism arises. Using a simulated RNA-like replicator system, we show that synergistic interaction between molecular replicators can occur spontaneously, given their adsorption on a (mineral) surface and the high degree of neutrality in RNA evolution (Schuster et al. 1994). Chemical properties of the modeled replicator such as growth/decay rates and catalytic capacity depend on RNA secondary structure (and active sites). We study the evolution of a system, initialized with a population of random sequences, towards two target structures assumed to have a specific catalytic activity. After a very long lag phase where non-functional replicators dominate the system, we observe a rapid transition towards metabolic cooperation of catalytically functional molecules. We conclude that partial compartmentalization by absorption on a surface, together with the neutrality in sequence-structure folding, suffices to enable the spontaneous and irreversible discovery of the first major transition.

Gilbert, W.: 1986, The RNA World, Nature 319, 618.

Joyce, G. F. and Orgel, L. E.: 1999, Prospects for Understanding the Origin of the RNA World, in Gesteland, R. F., Cech, T. R. and Atkins, J. F. (eds), The RNA World, pp. 49-77, Cold Spring Harbor Lab. Press, Cold Spring Harbor.

Maynard Smith, J. and Szathmáry, E.: 1995, The Major Transitions in Evolution, Freeman, Spektrum, Oxford.

Schuster P., Fontana, W., Stadler, P.F. and Hofacker, I.L.,1994, From sequences to shapes and back: a case study in RNA secondary structures. Proc. Royal Society London B, 255:, 279-284.

E-mail: sergio.branciamore@unifi.it

\section{A Kinase Ribozyme that Self-Phosphorylates at Two Different Sites}

\section{${ }^{1}$ Elisa Biondi, ${ }^{2}$ David Nickens, ${ }^{3}$ James Patterson, ${ }^{1,3}$ Dayal Saran, ${ }^{1}$ Donald Burke}

${ }^{1}$ Department of Molecular Microbiology \& Immunology and Department of Biochemistry, University of Missouri School of Medicine, 1201 E. Rollins St., Columbia, MO 65211- 
7310; ${ }^{2}$ Department of Biology, Indiana University, Bloomington, IN, 47405; ${ }^{3}$ Department of Chemistry, Indiana University, Bloomington, IN, 47405

Our long-term goal is to understand the catalytic potential of RNA, the feasibility of RNA-based evolution in an RNA World, and the possibility of using RNA to engineer artificial gene regulation and metabolism. A key constraint in the acquisition of new biochemical function is the interplay between substrate binding and catalysis. Simply put, active sites within metabolic ribozymes must accommodate diffusible substrates. We are analyzing the mechanism of action and catalytic requirements of kinase ribozymes. RNAcatalyzed phosphorylations are attractive to study for several reasons. First, phosphoryl transfer is one of the most important and ubiquitous reactions in small molecule and protein metabolism, and of fundamental biological and evolutionary significance. Second, the chemical mechanism of many natural kinases have been studied extensively, facilitating comparison of ribozyme and protein catalysis of equivalent reactions. Third, the underlying chemistry of phosphorylation reactions is relatively simple and can be easily examined.

We have selected several populations of self-phosphorylating ribozymes that utilize ATP (gammaS) or GTP(gammaS) as (thio)phosphoryl donor. Individual ribozymes are specific for one donor or the other, even for selections in which both donors were present. Mapping the sites of modification for several ribozymes identified one RNA with an especially complex active site that promotes phosphorylation of two distinct $2^{\prime}$ hydroxyls. These two sites are widely separated in primary sequence, and are presumed to be juxtaposed in the three dimensional structure of the RNA. A smaller version of this ribozyme-generated by systematic deletions of superfluous nucleotides - maintained the double-site catalytic activity and enhanced overall activity. We will present new data and further analysis of the structure and mechanism of this ribozyme.

E-mail: biondie@missouri.edu 


\section{Precellular Models and Early Biological Evolution}

\section{Chemical Synthetic Biology}

Luisi P.L. ${ }^{1}$, Stano P. ${ }^{1,2}$, De Lucrezia D. ${ }^{2,1}$, Wieczorek R. ${ }^{2,1}$, Chiarabelli C. ${ }^{1,2}$

${ }^{1}$ Department of Biology, University of Roma TRE, Rome, Italy; ${ }^{2}$ ECLT, European Center for Living Technology, Venice, Italy

In general terms, synthetic biology is concerned with the synthesis of life forms alternative to the extant ones, and in addition to DNA recombination and genome mixing, the field also enjoys the presence of a more chemical approach: the study of alternative biochemical structures at the level of macromolecules, proteins and RNAs in particular; or the chemical construction of cellular compartments alternative to the biological cells. This approach can be used for the origin of life, with emphasis to those structures that might have existed in the prebiotic chemical evolution. This form of synthetic biology is usually referred to as chemical synthetic biology, and a few examples will be presented here.

One first example concerns the "never born proteins" (NBP), proteins namely that are not with us on Earth because evolution has not produced them. The related, important question, is how and why the "few" extant proteins have been selected out. Perhaps "our" proteins have particular physical properties (folding, solubility, hydrodynamic properties,...)? A large library of NBP with 50 amino acid residues has been prepared by phage display, it has been checked that they have no similarity with the known proteins, and that, surprisingly, they have a very high frequency of folding. The comparison with "our" proteins reveals then that our proteins are not at all particular in terms of folding or thermodynamic stability or water solubility, which permits to say, tentatively, that our proteins are the product of contingency rather than a deterministic selection for their peculiar properties.

A second example of chemical synthetic biology concerns the prebiotic biogenesis of proteins. This strategy utilizes prebiotic peptides with catalytic activity for the synthesis of peptide bonds.

Finally, as third example, the project "minimal cells" will be illustrated. This is a project aimed at the laboratory construction of minimal living semi-synthetic cells, where minimal means that they have the minimal and sufficient number of components to be alive (metabolism, plus self-reproduction plus evolvability). They are realized with liposomes, into which extant genes and enzymes are incorporated. Liposomes containing the ribosomal kit and thus displaying the capability of protein expression have been realized by different laboratories. The state of art of this field will be analysed and discussed.

E-mail: luisi@mat.ethz.ch

\section{Self-Assembly and Polymerization in the Prebiotic Environment}

\section{David Deamer, Felix Olasagasti}

Department of Chemistry and Biochemistry, University of California, Santa Cruz CA95064

Although the physical environment that fostered primitive cellular life is still largely unconstrained, we can be reasonably confident that liquid water was required, together with a source of organic compounds and energy to drive polymerization reactions. There must also have been a process by which the compounds were sufficiently concentrated to 
undergo physical and chemical interactions. We are exploring the relationship between physical concentration, self-assembly processes and polymerization reactions of organic compounds in natural geothermal environments and related laboratory simulations. We have found that macromolecules such as nucleic acids and proteins are readily encapsulated in membranous boundaries during wet-dry cycles such as those that would occur at the edges of geothermal springs or tide pools. The resulting structures are referred to as protocells, in that they exhibit certain properties of living cells and are models of the kinds of encapsulated macromolecular systems that have the potential to evolve toward the first forms of cellular life. We have also determined that RNA-like polymers can be synthesized non-enzymatically from ordered arrays of mononucleotides in lipid microenvironments. Chemical activation of the mononucleotides is not required. Instead, synthesis of phosphodiester bonds is driven by the chemical potential of fluctuating anhydrous and hydrated conditions, with heat providing activation energy during dehydration. In the final hydration step, the RNA is encapsulated within lipid vesicles. We are now extending this approach to template-directed synthesis of short nucleic acid oligomers, in which lipidassisted polymerization serves as a laboratory model of replication in an RNA World.

E-mail: deamer@chemistry.ucsc.edu

\section{The Origins of Transmembrane Ion Channels}

Andrew Pohorille ${ }^{1,2}$, Michael A. Wilson ${ }^{1,2}$, Chenyu Wei ${ }^{1,2}$

${ }^{1}$ Exobiology Branch, NASA-Ames Research Center; ${ }^{2}$ Department of Pharmaceutical Chemistry University of California, San Francisco

How simple, protobiological macromolecules could have performed essential cellular functions that are currently carried out by complex proteins or protein assemblies remains one of the main, unresolved issues in the discipline of the origin of life. We will address this issue in the example of membrane proteins that mediate transport of ions across cell walls, a ubiquitous function that cannot be performed by RNA molecules. By combining results of experimental and computer simulation studies on synthetic models and natural channels, mostly of non-genomic origin, we show that the emergence of channels built of small, $\alpha$ helical peptides was protobiologically plausible, and did not require highly specific amino acid sequences. Despite their simple structure, such channels could possess properties that, at the first sight, appear to require markedly larger complexity. We will present our recent results for three types of channels that provide clues to the origin, mechanism of action and early evolution of ion channels. First, we will discuss model channels built of four, six and eight antimicrobial peptides, antiamoebin, and show how efficiency and selectivity of transport depend on the size of the pore. Next, we will illustrate in the example of M2 protein from the influenza virus how opening and closing a very simple, protontransporting channel can be regulated by changes in the conformation of just a few amino acid side chains. Finally, we will discuss regulation in a family of $\mathrm{pH}$ - and mechanosensitive channels that involves concerted movements of helices coupled with conformational changes in side chains. On the basis of our results, we propose that channels evolved towards high structural complexity because they needed to acquire mechanisms for precise regulation rather than to improve efficiency. In general, even though architectures of membrane proteins are not nearly as diverse as those of water-soluble proteins, they are sufficiently flexible to adapt readily to the functional demands arising during evolution.

E-mail: Andrew.Pohorille@nasa.gov 


\section{Evidence for a New Root of the Tree of Life}

James A. Lake ${ }^{1,2,3,4}$, Jacqueline A. Servin ${ }^{2,4}$, Craig W. Herbold ${ }^{2,4}$, Ryan G. Skophammer ${ }^{1,4}$

${ }^{1} \mathrm{MCD}$ Biology; ${ }^{2}$ Molecular Biology Institute; ${ }^{3}$ Human Genetics; ${ }^{4}$ UCLA Astrobiology Institute, University of California, Los Angeles, CA 90095, USA

A new root of the tree of life is providing evidence for a last common ancestor that is very different from the traditional one. This root provides a new perspective on the habitats of early life, including the evolution of methanogenesis, membranes, and thermophily; and the speciation of major prokaryotic taxa. Using indels, insertions and deletions, within paralogous genes our lab has obtained evidence for a new root to the tree of life in a series of recent papers. Through the analysis of indels present in 17 genes and their paralogous outgroups involved in diverse functions, including protein synthesis, DNA synthesis, heat shock responses, and nucleotide and amino acid synthesis, the root has been localized to a eubacterial branch of the tree between the clade consisting of the Actinobacteria and the double membrane (Gram negative) prokaryotes and the clade consisting of the Archaea and the Firmicutes. Since these results exclude the root from the archaeal-firmicute-clade, methanogenesis is excluded as a primitive prokaryotic metabolism. Mapping the phylogenetic distributions of genes involved in peptidoglycan- and lipidsynthesis onto this rooted tree parsimoniously implies that the ether archaeal lipids are not primitive, and that the cenancestral prokaryotic population consisted of organisms enclosed by a single, ester-linked lipid membrane, covered by a peptidoglycan layer. These results explain the similarities previously noted by others between the pathways of lipid synthesis in Bacteria and Archaea. Our results also imply the last common ancestor was not hyperthermophilic, although moderate thermophily cannot be excluded, consistent with the results of others.

Schopf, J.W. (2006) Fossil evidence of Archean life. Roy. Soc. Phil.Trans. Ser. B 361, 869885.

E-mail: Lake@mbi.ucla.edu

\section{Evolutionary Relationships of Bioenergetic Pathways}

\section{Lila Koumandou}

University of Cambridge, Department of Pathology, Tennis Court Road, Cambridge CB2 1QP, UK

Prokaryotes utilise an amazing diversity of bioenergetic pathways. These metabolic capabilities are suited to the variety of environments that prokaryotes inhabit, ensuring that organisms effectively utilise the redox potential of molecules found in their surroundings to harness energy for their survival. At the time of life's origin, the Earth probably contained a broad range of potentially habitable environments, but biological activity has also influenced the evolution of the Earth's surface environment. Molecular evolution studies, coupled to data from the geological record, indicate that the most primitive bioenergetic metabolisms were anaerobic and probably sulfur-dependent or methanogenic. The subsequent advent of oxygenic photosynthesis brought about a change in atmospheric oxygen levels, after which aerobic respiration and oxygen-requiring chemosynthetic pathways evolved. However, this 
variety of energy metabolisms evolved within a relatively short time ( 1 billion years) from the estimated origin of life on Earth and has since been mostly characterised by conservatism. Furthermore, these metabolic modes are not monophyletic, i.e. shared by a group of closely evolving relatives, but instead are mixed among different lineages within the proteobacteria and the archaea. So, since this metabolic diversity evolved early on in life, and is widespread among the bacteria and the archaea, I want to explore how these different bioenergetic pathways evolved. Did each pathway evolve independently, or did they all evolve from a simple ancestral metabolism? And if the latter is the case, what was the first energy source used by life? As in morphological evolution, the evolution of new metabolic capabilities often occurs by the modification of pre-existing pathways. I will present a framework for testing the hypothesis that parts of bioenergetic pathways were co-opted to evolve into new pathways, using structural bioinformatics to look at deep evoltuionary relationships preserved in the protein structure of the core proteins involved.

Battistuzzi FU, Feijao A, Hedges SB (2004) A genomic timescale of prokaryote evolution: insights into the origin of methanogenesis, phototrophy, and the colonization of land. BMC Evol Biol 4: 44

Knoll AH, Bauld J (1989) The evolution of ecological tolerance in prokaryotes. Trans R Soc Edinb Earth Sci 80: 209-23

Reysenbach AL, Shock E (2002) Merging genomes with geochemistry in hydrothermal ecosystems. Science 296(5570): 1077-82

Rison SC, Thornton JM (2002) Pathway evolution, structurally speaking. Curr Opin Struct Biol 12(3): 374-82

Woese CR (1987) Bacterial evolution. Microbiol Rev 51(2): 221-71

E-mail:vk219@cam.ac.uk 


\title{
Astrobiology and Search for Life
}

\section{Adaptability of Halotolerant-Bacteria to Europa's Environment}

\author{
Horacio Terrazas ${ }^{1}$, Sandra I. Ramírez ${ }^{2}$, Enrique Sánchez ${ }^{3}$
}

${ }^{1}$ Facultad de Ciencias Biológicas; ${ }^{2}$ Centro de Investigaciones Químicas; ${ }^{3}$ Centro de Investigación en Biotecnología, Universidad Autónoma del Estado de Morelos, Av. Universidad No. 1001 Col. Chamilpa 62209 Cuernavaca, Morelos MEXICO

Extremophiles are distinguished by their capacity to develop basic metabolic activities in environments with physical and chemical harsh conditions where most of the mesophiles organisms cannot survive (Rothschild and Mancinelli, 2001). Halophiles are a particular type of extremophiles capable of living in moderate to high saline concentration values, extremely resistant to microgravity conditions and UV radiation exhibition, able to stay viable for long time periods within saline crystals and with a highly specialized biochemistry (Oren, 1999). These characteristics have stimulated the study on the viability to use halophiles as models in Astrobiology studies (Dassarma, 2006), particularly for the Europan satellite environment whose main characteristic is the presence of a deep liquid water ocean rich in salts $\left(\mathrm{NaCl}, \mathrm{MgSO}_{4}\right)$ with tidal forces occurring between the ocean and its thick ice cover (Marion et al. 2003). The objective of this study is to evaluate the capability of halotolerant bacteria to growth on laboratory conditions analogue to those of the Europan ocean surface. Experiments were designed to test the growth of halotolerant bacteria collected from a liquid industrial brine with salt contents of $6-10 \%(w / v)$ measured as $\mathrm{NaCl}$. The tested parameters were the highest limit of salinity, and proton concentration $(\mathrm{pH})$, as well as the lowest temperature limit. After a purification process and a detailed observation of morphological characteristics, the presence of three distinct stocks identified here as T806-1, T806-2, and T806-3 was confirmed. Further biochemical and molecular tests based on $16 \mathrm{~S}$ rRNA unit allowed a more detailed classification. A 10\% TSB culture medium was used in all cases and bacterial growth was monitored spectroscopically by determining the optical density at $600 \mathrm{~nm}$ and also by viable count tests performed at 48-h intervals. The experimental conditions can be summarized as follows: each one of the three stocks was grown in a culture medium enriched with $\mathrm{NaCl}, \mathrm{MgSO}_{4}$ and $\mathrm{Na}_{3} \mathrm{PO}_{4}$ at $2 \%, 5 \%$ and $10 \% \mathrm{w} / v$ concentration. The acidity of the culture medium was set at $\mathrm{pH}$ values of 2.0, 5.5 and 9.0 with a phosphate buffer. The Europa's ocean surface scenario was simulated using a hermetically isolated $100-\mathrm{mL}$ flask where $50 \mathrm{~mL}$ of the $10 \%$ TSB medium was inoculated with a combination of T806-1 and T806-3 strains and enriched with $5 \% \mathrm{NaCl}$ and $10 \% \mathrm{MgSO}_{4}$ at a $\mathrm{pH}$ value of 5.5. Tests were performed introducing 50 mbar of $5 \%, 10 \%$ and $20 \% v / v$ oxygen content balanced with argon.

Three different stocks were isolated and characterized. Two of them, T806-1 and T806-3 were perfectly able to grow in the presence of up to $10 \%$ of $\mathrm{NaCl}$ and $\mathrm{MgSO}_{4}$ and at an acidity value of 5.5. These conditions have specific relevance to the Europan ocean. Their growth showed the capability of these bacteria to adapt to high contents of salts. The halotolerant bacteria have also demonstrated their capability to resist short exposures to low temperatures (below the water freezing point), after which they continue viable. The implications of all these results in the frame of a salty Europan ocean will be presented and discussed.

We thank Concepción Chino for help with sequencing and analysis of 16S rRNA. This work was supported through a CONACyT 52291 grant. 
Dassarma, Shiladitya, (2006). Extreme Halophiles are models for Astrobiology. Microbe, 1 (3).

Marion, G., Fritsen, C., Eicken, H., and Payne, M. (2003). The search for life on Europe: Limiting environmental factors, potential habitats, and Earth analogues. Astrobiology, 3 (4):785-811.

Oren, A. (1999). Bioenergetic aspects of halophilism. Microbiol. Mol. Biol. Rev. 63: 334348.

Rothschild, L. J. and Mancinelli, R. L. (2001). Life in Extreme Environments. Nature, 409: 1092-1101.

E-mail: ramirez_sandra@ciq.uaem.mx

\section{Extraterrestrial Nucleobases in the Murchison Meteorite}

Zita Martins ${ }^{1,2}$, Oliver Botta ${ }^{3,4,5}$, Marilyn L. Fogel ${ }^{6}$, Mark A. Sephton ${ }^{2}$, Daniel P. Glavin ${ }^{3}$, Jonathan S. Watson ${ }^{7}$, Jason P. Dworkin ${ }^{3}$, Alan W. Schwartz ${ }^{8}$, Pascale Ehrenfreund ${ }^{1,3}$

${ }^{1}$ Astrobiology Laboratory, Leiden Institute of Chemistry, Leiden, The Netherlands;

${ }^{2}$ Department of Earth Science and Engineering, Imperial College London, UK; ${ }^{3}$ NASA Goddard Space Flight Center, Code 699, Greenbelt, USA; ${ }^{4}$ Goddard Earth Sciences and Technology Center, Univ. Maryland, Baltimore, USA; ${ }^{5}$ International Space Science Institute, Bern, Switzerland; ${ }^{6} \mathrm{GL}$, Carnegie Institution of Washington, Washington, USA; ${ }^{7}$ Planetary and Space Sciences Research Institute, The Open University, Milton Keynes, UK; ${ }^{8}$ Radboud University Nijmegen, The Netherlands

Carbon-rich meteorites, also known as carbonaceous chondrites, contain many biologically relevant organic molecules and delivered prebiotic material to the young Earth. Nucleobases, which are important compounds in modern terrestrial biochemistry, have been detected in carbonaceous chondrites by several research groups. Because significant quantitative and qualitative differences were observed (even within the same meteorite), the extraterrestrial origin of these nucleobases was subject to confirmation. In order to address this crucial question, we have performed for the first time compound-specific carbon isotope measurements for nucleobases (one purine and one pyrimidine) present in the Murchison meteorite, using gas chromatography-combustion-isotope ratio mass spectrometry (GC-C-IRMS). Carbon isotope ratios for uracil and xanthine of $\delta^{13} \mathrm{C}=+44.5 \mathrm{o} / \mathrm{oo}$ and $+37.7 \mathrm{o} / \mathrm{oo}$, respectively, unambiguously confirm a non-terrestrial origin of these compounds. These new results demonstrate that organic compounds, which are components of the genetic code in modern biochemistry, were already present in the early Solar System and may have played a key role in life's origin.

E-mail: p.ehrenfreund@chem.leidenuniv.nl 


\title{
POSTERS
}

\section{Planetary Evolution}

\section{Detection of Cometary Amines in Samples Returned by the Stardust Spacecraft}

\author{
Daniel P. Glavin ${ }^{1}$, Jason P. Dworkin ${ }^{1}$, J. E. Elsila ${ }^{1}$, Scott A. Sandford ${ }^{2}$
}

\author{
${ }^{1}$ NASA Goddard Space Flight Center, Greenbelt MD 20771, USA; ${ }^{2}$ NASA Ames Research \\ Center, Moffett Field CA 94035, USA
}

The delivery of amino acids to the early Earth by comets and their fragments could have been a significant source of the early Earth's prebiotic organic inventory that led to the emergence of life (Chyba and Sagan, 1992). Over 20 organic molecules including methane, ethane, ammonia, cyanic acid, formaldehyde, formamide, acetaldehyde, acetonitrile, and methanol have been identified by radio spectroscopic observations of the comae of comets Hale-Bopp and Hyakutake (Crovisier et al. 2004). These simple molecules could have provided the organic reservoir to allow the formation of more complex prebiotic organic compounds such as amino acids.

After a 7-year mission, the Stardust spacecraft returned to Earth samples from comet Wild 2 on January 15, 2006 providing the opportunity to analyze the organic composition and isotopic distribution of cometary material with state-of-the-art laboratory instrumentation. The Preliminary Examination Team analyses of organics in samples returned by Stardust were largely focused on particles that impacted the collector aerogel and aluminum foil (Sandford et al. 2006). However, it is also possible that Stardust returned a "diffuse" sample of gas-phase organic molecules that struck the aerogel directly or diffused away from the grains after impact. To test this possibility, samples of Stardust flight aerogel and foil were carried through a hot water extraction and acid hydrolysis procedure to see if primary amine compounds were present in excess of those seen in controls. Here we report highly sensitive liquid chromatography time-of-flight mass spectrometry measurements of amino acids and amines in samples returned from a comet (Glavin et al. 2008).

A suite of amino acids and amines including glycine, L-alanine, methylamine (MA), and ethylamine (EA) were identified in the Stardust bulk aerogel. With the exception of MA and EA, all other primary amines detected in comet-exposed aerogels were also present in the aerogel witness tile that was not exposed to Wild 2, suggesting that most amines are terrestrial in origin. However, the enhanced abundances of MA, EA, and possibly glycine in comet-exposed aerogel compared to controls, coupled with MA to EA ratios (1 to 2) that are distinct from preflight aerogels (7 to 10), suggest that these amines were captured from Wild 2. It is possible that MA and EA were formed on energetically processed icy grains containing methane, ethane, and ammonia. The presence of cometary amines in Stardust material supports the hypothesis that comets were an important source of prebiotic organics on the early Earth. To better understand their origin, a systematic compound specific carbon isotopic analysis (C-CSIA) via gas chromatography quadrupole mass spectrometry in with parallel with combustion isotope ratio mass spectrometry (GCQMS/IRMS) is being conducted. We will discuss our latest C-CSIA measurements and what they indicate about the origin of amino acids extracted from Stardust samples. 
Chyba, C. F. and Sagan, C. (1992) Endogenous production, exogenous delivery, and impact-shock synthesis of organic molecules: an inventory for the origins of life. Nature, 355: 125-132.

Crovisier, J., Bockele-Morvan, D., Colom, P., Biver, N., Despois, D., Lis, D. C., and the Team for Target-of-Opportunity Radio observations of Comets. (2004) The composition of ices in comet C/1995 O1 (Hale-Bopp) from radio spectroscopy. Further results and upper limits on undetected species. Astron. Astrophys. 418: 1141-1157.

Glavin, D. P., Dworkin, J. P., and Sandford, S. A. (2008) Detection of cometary amines in samples returned by Stardust. Meteorit. Planet. Sci. 43: 399-414.

Sandford, S. A. et al. (2006) Organics captured from comet 81P/Wild 2 by the Stardust spacecraft. Science, 314: 1720-1724.

E-mail: daniel.p.glavin@nasa.gov

\section{Gamma-Ray Bursts and Giant Flares Effects on the Early Evolution of the Biosphere}

\section{J. E. Horvath, D. Galante}

\section{IAG-USP, Sao Paulo U}

We present in this talk a unified, quantitative synthesis of analytical and numerical calculations of the effects caused on an Earth-like planet by a Gamma-Ray Burst (GRB) and nearby giant flares from Soft-Gamma Repeaters, considering atmospheric and biological implications (Thomas \& Mellot, 2006). The main effects of a GRB/giant flare are classified in four distinct types and analyzed separately, namely the direct radiation transmission, UV flash, ozone layer depletion and cosmic rays. The "effectiveness" of each of these effects is compared and critical distances for significant biological damage are given for each one (Galante \& Horvath, 2007).

We find that the first three effects have potential to cause global environmental changes and biospheric damages, even if the source is located at great distances (from a few to hundred kpc, see Thorsett 1995), however, cosmic rays would only be a serious threat for very close GRB sources. Therefore, the question of the rate of events along the history of the galaxy has to be considered, and the importance of the search for signatures stressed (Scalo \& Wheeler, 2002). In the case of the rare, nearby sources SGR we evaluate, using the same criteria for the softer spectra and other observed features (which greatly helps for the assessment of actual damages), the probability of a giant flare within a given distance. The result is that this class of sources should be considered as a substantial biological agent giving radiation "jolts" to the biota affected by their incidence.

Galante, D.; Horvath, J.E. (2007). Biological effects of gamma-ray bursts: distances for severe damage on the biota. Int. Jour. Astrobiology 6: 19-26

Scalo, J.;Wheeler, J.C.(2002). Astrophysical and Astrobiological Implications of GammaRay Burst Properties. ApJ, 566: 723-737.

Thomas, B. C., Melott, A.L., (2006). Gamma-ray bursts and terrestrial planetary atmospheres. New Jour. Phys., 8: 120-129

Thorsett, S. (1995). Terrestrial implications of cosmological gamma-ray burst models. ApJL, 444:L53-L55.

E-mail: foton@astro.iag.usp.br 


\section{Spectroscopic Investigations of High-Power Laser Sparks in Gas Mixtures Containing Methane: A Laboratory Model of Energetic Events in Strongly Reduced Planetary Atmospheres}

Svatopluk Civiš ${ }^{1}$, Martin Civiš ${ }^{2}$, Robin Rašín ${ }^{2}$, Michal Kamas ${ }^{1}$, Kseniya Dryahina ${ }^{1}$, Patrik $\breve{S}_{\text {panĕl}}{ }^{1}$, Libor Juha ${ }^{2}$, Martin Ferus ${ }^{1,2}$

${ }^{1}$ J. Heyrovsky Institute of Physical Chemistry, Czech Academy of Sciences, Dolejškova 3, 18223 Prague 8, Czech Republic; ${ }^{2}$ Institute of Physics, Czech Academy of Sciences, Na Slovance 2, 18221 Prague 8, Czech Republic

Single short $(0.5 \mathrm{~ns})$ pulses with high energy content $(\leq 1 \mathrm{~kJ})$ provided by a high-power laser are focused into molecular gases to create large laser sparks (Civis et al., 2004). This provides a unique way to mimic the chemical effects of high-energy-density events in planetary atmospheres (cometary impact, lightning) matching the natural energy-density and plasmavolume scaling of such events in a fully-controlled laboratory environment. The many chemical reactions initiated by the laser-induced dielectric breakdown (LIDB) in both pure molecular gases and in their mixtures with the compositions related to the study of the chemical evolution of the Earth's early atmosphere are systematically studied. The processes responsible for the chemical action of laser sparks are identified and investigated (Babankova et al., 2006a and b).

FTIR spectrometer Bruker IFS $120 \mathrm{HR}$ was used for analysis of chemical changes in the irradiated gas mixtures. This method is very useful for the detection of isotopic exchange in the studied systems. The $\mathrm{C}_{2} \mathrm{D}_{2}$ and $\mathrm{C}_{2} \mathrm{DH}$ molecules were detected in specific ratios.

The complementary analytical methods GC-MS and SIFT-MS were used. Organic molecules such ethene, propane and propene, propadiene, pentadiene, propine, hydrogencyanide, methanole, n-butene, ethanole, acetone, isopropanole and cyanoacetylene have been detected in the irradiated mixture of $\mathrm{CH}_{4}-\mathrm{N}_{2}-\mathrm{D}_{2} \mathrm{O}$.

Babankova, D., S. Civis, L. Juha: Chemical consequences of laser-induced breakdown in molecular gases, Prog. Quant. Electron. 30, 75 (2006a).

Babankova, D., S. Civis, L. Juha, M. Bittner, J. Cihelka, M. Pfeifer, J. Skala, A. Bartnik, H. Fiedorowicz, J. Mikolajczyk, L. Ryc, T. Sedivcova: Optical and X-ray emission spectroscopy of high-power laser-induced dielectric breakdown in molecular gases and their mixtures, J. Phys. Chem. A110, 12113 (2006b).

Civis, S., L. Juha, D. Babankova, J. Cvacka, O. Frank, J. Jehlicka, B. Kralikova, J. Krasa, P. Kubat, A. Muck, M. Pfeifer, J. Skala, J. Ullschmied: Amino acid formation induced by a high-power laser in $\mathrm{CO}_{2} / \mathrm{CO}-\mathrm{N}_{2}-\mathrm{H}_{2} \mathrm{O}$ gas mixtures, Chem. Phys. Lett. 386, 169 (2004).

This work was financially supported by Grant Agency of the Czech Republic (grant No. 203/06/1278) and the Czech Ministry of Education (grants LC510 and LC528).

E-mail: martin.ferus@seznam.cz

\section{Hypothesis of Formation of Planets from Nebula: Why Are the Planets Different in Their Chemical Compositions?}

\section{E. Ostrovskii ${ }^{1}$, E. A. Kadyshevich ${ }^{2}$}

${ }^{1}$ Karpov Inst. Phys. Chem., Moscow, Russia; ${ }^{2}$ Obukhov Inst. Atmosph. Phys., Moscow, Russia 
Most planetologists believe that the Solar System originated from a nebula (a giant plasma cloud) (Shmidt, 1949; Hoyle, 1981), which arouse as a result of the supernova explosion about 4.6 billion years ago. More than $99 \%$ of nebular atoms were $\mathrm{H}$ and $\mathrm{He}$. Several models (e.g., Jang-Condell and Boss, 2007; Boss, 2008; Alibert, et al., 2005) were proposed for simulating the processes of planet formation. However, neither the history, nor the physics and chemistry of planet formation are known in detail. There is an opinion that the radius of a planet is the key parameter controlling most of its evolutional features (Albarède and Blichert-Toft, 2007). Meanwhile, a planet radius may be time-dependent and the character of this dependence can not be now specified reliably. The possibility for correlation of models proposed for description of planet formation with the actual transformations of remote stellar systems became available only recently. The evolution causes of the principal differences in the mineral composition and chemical and physical properties of the planets are not yet clarified.

This presentation is an attempt to explain these differences on the basis of a phenomenological model containing new elements. We subdivide the Solar System objects into the physically formed objects (PFO) formed in the cold region of the nebula (from the outside to the present objects of the Main Asteroid Belt) and chemically formed objects (CFO) formed in the hot region of it (Kadyshevich, Ostrovskii, in press). After the big bang, nebula expanded quickly and cooled steadily. In this period, $\mathrm{H}_{2}$ molecules and hydride radicals and molecules with the bond energy exceeding that in $\mathrm{H}_{2}$ (per $\mathrm{H}$ g-atom) formed. With time, nebula transformed to a flat thin disk composed of many concentric diffusely-bounded rings; the more peripheral they were, the lighter molecules they tended to contain. PFO formation started, when the nebula began to collapse after its outer $\mathrm{H}_{2}$ and $\mathrm{He}$ rings cooled to the $\mathrm{H}_{2}$ condensation temperature; $\mathrm{H}_{2}$ droplets absorbed light $\mathrm{Li}, \mathrm{Be}, \mathrm{B}, \mathrm{LiH}$, and $\mathrm{BeH}$ atoms and molecules, which formed the agglomerate cores and increased their size competing with each others for the mass and gravitational attraction. Heavy atoms and hydrides remained in that nebula section in which the temperature was too high for their physical agglomeration and in which their concentration was too low for chemical reactions to proceed to a significant degree. As the nebular-disc compression increased, chemical combination reactions accelerated in the diffusive regions of the neighboring disc rings, exponentially stimulated localizations of the substances and reaction heat, and initiated compressible vortexes, within which hot cores of the present sky objects localized. This heat was capable of melting the cores but was not capable of their evaporating. The pressure depletion in the vicinities of the giant vortexes and the gravitational attraction of the last stimulated flows of light cold vaporous and gaseous substances and their asteroid-like agglomerates from the outer space and also of asteroid-like agglomerates of not so light substances from the intermediate regions of the space to the hot cores originated by the vortexes. The flows precipitated over the hot core surfaces of the CFO and cooled these surfaces. The sandwiches obtained as a result of this precipitation became steadily the young Earth-group planets and their satellites. These mechanisms are capable of explaining the planet compositions.

Alibert, Y. et al. (2005). Models of giant planet formation with migration and disc evolution. $A \& A, 434$ : 343-353.

Albarède F. and Blichert-Toft, J. (2007). Comptes Rendus Geoscience, 339(14-15): 917-929 Boss, A.P. (2008). diffusion approximation models of giant planet formation by disk instability. The Astrophysical Journal, 677(1):607-615.

Hoyle, F. (1981). The big bang astronomy. New Scientist, 92:521-527. 
Jang-Condell, H. and Boss, A.P. (2007). Signatures of planet formation in gravitationally unstable disks. The Astrophys. J. Letters, 659:L169-L172.

Kadyshevich, E. A. and Ostrovskii V. E. (in press). Planet-system origination and methanehydrate formation and relict atmosphere transformation at the Earth. To appear in Izvestiya, Atmospheric and. Oceanic Physics.

Shmidt, O. Yu. (1949). Four lectures on the Earth-formation theory. Acad. Sci. USSR, M. (Rus.)

E-mail: vostrov@cc.nifhi.ac.ru

\title{
Life Origination Hydrate Hypothesis (LOH-Hypothesis)
}

\author{
V. E. Ostrovskii ${ }^{1}$, E. A. Kadyshevich ${ }^{2}$ \\ ${ }^{1}$ Karpov Inst. Phys. Chem., Moscow, Russia; ${ }^{2}$ Obukhov Inst. Atmosph. Phys., Moscow, \\ Russia
}

One of the first scientific hypotheses of living matter origination was proposed by Oparin (1952). It was picked up and developed by Urey, Miller and their colleagues (e.g., Miller and Urey, 1959). Later, the idea about primary development of a RNA world and its subsequent reformation into present DNA/RNA world was developed. Important contributions to these ideas were made by Orgel, Kauffman, Joyce and others (e.g., Miller and Orgel, 1974; Kauffman, 1993; Joyce, 1989). At present, these ideas and the idea of Panspermia are widely distributed.

We develop the original Life Origination Hydrate Hypothesis (LOH-hypothesis) (Ostrovskii and Kadyshevich, 2002; 2006; 2007) assuming repeated formation of livingmatter simplest elements (LMSE) within honeycomb structures of hydrocarbon-hydrates from $\mathrm{CH}_{4}$ (or other hydrocarbon), niter, and phosphate under the Earth's surface or seabed in the following sequence: niter diffusion into hydrate structure $\rightarrow$ formation of N-bases and riboses within large structural cavities $\rightarrow$ phosphate diffusion from outside into small structural cavities $\rightarrow$ formation of DNA- (RNA-) like molecules through polymerization $\rightarrow$ melting of the system and water-organic-soup formation $\rightarrow$ formation of amino-acids and simplest organelles in the soup $\rightarrow$ self-replication of nucleic acids and concentrating of the soup $\rightarrow$ formation of cells etc. The LOH-hypothesis is supplemented with the subhypothesis of formation of deposits of hydrates of $\mathrm{CH}_{4}$ and other hydrocarbons. The mechanisms for each step are proposed and discussed. The LOH-hypothesis was initiated by results of our calorimetric studies of water sorption-desorption processes in systems modelling interaction between water and biologically-active substances, by surprising coincidence between the sizes of hydrate structural cavities and N-bases, riboses, and phosphates, and by analysis of available works relating to the living-matter-origination problem. Thermodynamic calculations supporting the LOH-hypothesis, a new supposition allowing for understanding the homochirality of nucleic acids, a plan of a PC experiment examining this supposition, and the scheme for a laboratory experiment capable of testing the LOH-hypothesis are presented. The simplicity of the acts of Nature is an attribute of our hypothesis: the entire set of the necessary LMSE and of protocells formed simultaneously and in the same place. Phenomena counting in favour of our hypothesis are described (e.g., Schippers et al., 2005).

The LOH-hypothesis allows for answering the following questions. From what substances and by what mechanism had the LMSE originated? In what way had these substances met in one place, and why had no other substances reacted with them? Why are 
the DNA and RNA monomer links limited in size, and why are they so similar? How had it happened that the sequences of N-bases in DNA and RNA molecules are not random? What had hampered the subsequent chemical transformations of the rings and side groups of the N-bases and riboses? Why do atoms other than $\mathrm{C}, \mathrm{N}, \mathrm{P}, \mathrm{O}$, and $\mathrm{H}$ almost never enter the DNA and RNA compositions? Why do only five N-bases usually participate in DNA and RNA formation, and why do some other N-bases, e.g., xanthine, sometimes enter the DNA and RNA compositions?

Joyce, G. F. (1989). RNA evolution and the origins of life. Nature, 338:217-224.

Kauffman, S. (1993). The Origin of Order Self Organization and Selection in Evolution. Oxford Univ. Press, Oxford.

Miller, S. L. and Orgel, L. E. (1974). The Origin of Life on the Earth. Prentice-Hall, Englewood Cliffs, N.Y.

Miller, S. L. and Urey, H. C. (1959) Organic compound synthesis on the primitive Earth: Several questions about the origin of life have been answered, but much remains to be studied. Science, 130:245-251.

Oparin, A. I. (1952) The Origin of Life. Dover, New York.

Ostrovskii, V.E., Kadyshevich E. A. (2002). Hydrate model of the DNA- $\mathrm{H}_{2} \mathrm{O}$ system. Int. J. Nanosci., 1:101-121.

Ostrovskii, V. E. and Kadyshevich, E. A. (2006). Thermodynamics of formation of nitrogen bases and D-ribose from mineral substances in light of the problem of origination of simplest elements of living matter. Thermochim. Acta, 441:69-78.

Ostrovskii, V. E. and Kadyshevich E. A. (2007). Generalized hypothesis of the origin of the living-matter simplest elements, transformation of the Archean atmosphere, and the formation of methane-hydrate deposits. Physics-Uspekhi, 50:175-196.

Schippers, A. et al. (2005). Prokaryotic cells of the deep sub-seafloor biosphere identified as living bacteria. Nature, 433: 861-864.

E-mail: vostrov@cc.nifhi.ac.ru;victor@ostrovskiy.net

\title{
Atmospheres of Early Noachian Mars and Early Archean Earth
}

\author{
Feng Tian ${ }^{1}$, James F. Kasting ${ }^{2}$
}

${ }^{1}$ MIT (after July 6); ${ }^{2}$ Penn State University

The atmosphere of early Earth could have been the environment where prebiotic molecules were formed efficiently (Miller 1953). Alternatively, these compounds could have been delivered to early Earth by exogenous sources (Chyba and Sagan 1992, Martins et al. 2008). The first channel would have been efficient in providing these building blocks of life IF the atmosphere of early Earth was highly reduced; however, the early Earth's atmosphere is generally considered to have been neutral or weakly reduced (Walker 1977, Kasting 1993). A single-component hydrodynamic escape model (Tian et al. 2005) suggested that a hydrogenrich atmosphere could have been maintained on early Earth, although the one-species nature of the model and the lack of treatment of nonthermal escape processes weakened this conclusion. New multi-component hydrodynamic thermosphere-ionosphere models (Tian et al. 2008a, b) have been developed to account for the shortcomings of the earlier work and will be applied to revisit the problem of hydrogen escape from the early Earth.

We also present new numerical calculations for a dense, $\mathrm{CO}_{2}$-rich atmosphere on early Mars. In particular, we have found that the weak gravity field of Mars, in combination with 
the strong solar EUV radiation of a young Sun, makes the thermal escape of atomic carbon and oxygen efficient. As a result, it would have been difficult for early Mars to have maintained a dense $\mathrm{CO}_{2}$ atmosphere prior to 4.1 billion years ago (Tian et al. 2008c). Inclusion of a parameterized nonthermal escape process at the exobase level consumes more energy and leads to a dramatically different upper atmosphere structure. The overall escape rate of the dominant gases at the exobase level is conserved, regardless of whether nonthermal loss processes were efficient (Tian and Kasting 2008). We will speculate about what the Mars calculations imply about early Earth's atmosphere.

Chyba, C., C. Sagan (1992) Endogenous production, exogenous delivery and impact shock synthesis of organic molecules: an inventory for the origins of life. Nature 355, 125-132. Kasting, J.F. (1993) Earth's early atmosphere. Science 259, 920-926.

Martins, Z. et al. (2008) Extraterrestrial nucleobases in the Murchison meteorite. Earth and Planetary Science Letters 270, 130-136.

Miller, S.L. (1957) A production of amino acids under possible primitive Erath conditions. Science $117,528-529$.

Tian, F., O.B. Toon, A.A. Pavlov, H. De Sterck (2005) A hydrogen-rich early Earth atmosphere. Science, 308, 1014-1017.

Tian, F., J.F. Kasting, H. Liu, R.G. Roble (2008a) Hydrodynamic planetary thermosphere model. I: the response of the Earth's thermosphere to extreme solar EUV conditions and the significance of adiabatic cooling, JGR-planets 113, E05008 doi:10.1029/2007JE002946.

Tian, F., S.C. Solomon, L. Qian, J. Lei, R.G. Roble (2008b) Hydrodynamic planetary thermosphere model. II: coupling of an electron transport/energy deposition model. JGRplanets, in press

Tian, F., J.F. Kasting, S.C. Solomon (2008c) Fast thermal escape of carbon and oxygen from a dense, CO2-ruch early Martian atmosphere. Science, under review.

Tian, F. and J.F. Kasting (2008) Invariance of the total atmosphere escape rate from the atmosphere of early Mars. GRL, in preparation.

Walker, J.C.G. (1977) Evolution of the atmosphere. Macmillan, New York.

E-mail: feng.tian@colorado.edu 


\title{
Prebiotic Syntheses
}

\section{A Prebiotic Surface-Catalysed Formation of Alkyl Imines}

\author{
Nigel Aylward
}

School of Physical and Chemical Sciences Queensland University of Technology George St., Brisbane, Queensland 4000 AUSTRALIA

Alkynes such as ethyne form weak charge-transfer, ${ }^{11}$-alkenyl (vinyl) complexes (Collman et al., 1987) with surface catalysts such as Mg.porphin in which the alkenyl group has a net positive charge, and the conjugated porphin has a negative charge. The enthalpy change for the complex formation is small $(-0.002 \mathrm{~h})$. This neutral complex is polarised and undergoes a nucleophilic addition reaction with ammonia at the carbene carbon to form Mg.2-amino ethenyl (vinyl).porphin with a small enthalpy change $(0.016 \mathrm{~h})$. The complex has a tendency to cyclycise, and with an activation energy of, $0.058 \mathrm{~h}$ undergo a prototropic shift to yield the Mg.aziridinyl.porphin complex. The enthalpy change is favourable, $-0.004 \mathrm{~h}$. A further tropic shift with an activation energy of $0.111 \mathrm{~h}$ leads to ring opening, also with a favourable enthalpy change of $-0.015 \mathrm{~h}$. The ligand is then bound as a Mg.acetaldimine(ethanimine).porphin complex. This mechanism constitutes another mechanism for the formation of reactive, and unstable, imines that could facilitate the formation of aziridine-2ones, which have been predicated as important in amino-acid synthesis (Aylward and Bofinger, 2001). The reactions have been shown to be feasible from the overall enthalpy changes in the ZKE approximation at the HF and MP2 /6-31G* level.

Aylward, N.N and Bofinger, N, OLEB,6,2001. pp481-500

Collman J.P., Hegedus, L.S., Norton, J.R., Finke, G., Principles and Applications of Organotransition Metal Chemistry, University Science books, Mill Valley, California, 1987 pp525-608.

E-mail: n.aylward@student.qut.edu.au

\section{On the Possible Role of Metastable Excited Atoms in the Chemical Evolution of Planetary Atmospheres: A Laboratory Investigation by the Crossed Molecular Beam Technique}

Nadia Balucani, Raffaele Petrucci, Francesca Leonori, Piergiorgio Casavecchia

Dipartimento di Chimica, Università degli Studi di Perugia, Perugia, Italy

In our laboratory we have used the crossed molecular beam (CMB) technique with mass spectrometric (MS) detection to investigate elementary reactions of relevance in the chemistry of planetary atmospheres for a number of years. The main advantage of CMB experiments is that it is possible to observe the consequences of well defined molecular collisions and avoid the effects of secondary or wall collisions (Balucani, et al. 2006). The quantities observable by this experimental technique allow us to achieve the most detailed characterization of a gasphase reaction and to derive important features, such as the product branching ratios. In this respect, the coupling of the $\mathrm{CMB}$ technique with MS detection is crucial, because every product species can be ionized at the electron energy used in the ionizer which precedes the 
mass filter and so detected. By using the $\mathrm{CMB} / \mathrm{MS}$ technique we have been able to fully characterize some reactions of relevance in astrochemistry involving atomic species - such as O, C and N (Balucani, et a1. 2006; Costes, et al. 2006; Balucani and Casavecchia, 2006) - or simple radicals - such as $\mathrm{CN}$ and $\mathrm{OH}$ (Casavecchia, et al., 2001) - or unstable closed-shell species - such as $\mathrm{C}_{2}$ (Leonori, et al. 2008).

In this contribution, the attention will be focused on several reactions involving electronically excited, metastable states of atomic species-namely $\mathrm{C}\left({ }^{1} D\right), \mathrm{N}\left({ }^{2} D\right), \mathrm{O}\left({ }^{1} D\right)$ and $\mathrm{S}\left({ }^{1} D\right)$. In all cases, the radiative lifetime - spanning the range from $30 \mathrm{~s}$ for $\mathrm{S}\left({ }^{1} D\right)$ to $48 \mathrm{~h}$ for $\left.\mathrm{N}^{2} D\right)$ - is long enough to allow for bimolecular reactions to occur, provided that the gas density is not too low. All the above mentioned excited states can be formed in upper planetary atmospheres from simple parent molecules (either by photodissociation induced by solar photons or electron impact dissociation and dissociative recombination) and so contribute to the chemical evolution of planetary atmospheres. This is especially true as the excited atoms are much more reactive than those in the ground states, particularly when the reacting partner is a saturated molecules such as methane (in this case, for instance, the reactions involving the excited states have rate constants larger by 3-4 orders of magnitude). The role of $\mathrm{O}\left({ }^{1} D\right)$ and $\mathrm{N}\left({ }^{2} D\right)$ in the terrestrial atmosphere is indeed well assessed. In particular, we have investigated the reactions of $\mathrm{N}\left({ }^{2} D\right), \mathrm{C}\left({ }^{1} D\right)$ and $\mathrm{S}\left({ }^{1} D\right)$ with simple hydrocarbons relatively abundant in the gaseous environments of our solar system, i.e. methane, acetylene and ethylene. We have observed in all cases the formation of molecules containing a novel $\mathrm{C}-\mathrm{X}$ bond (where $\mathrm{X}=\mathrm{C}, \mathrm{N}, \mathrm{S}$ ). Some reactions will be illustrated including the reactions $\mathrm{C}\left({ }^{1} D\right)+\mathrm{CH}_{4}$, which contributes in converting methane to acetylene, and $\mathrm{S}\left({ }^{1} D\right)+\mathrm{C}_{2} \mathrm{H}_{2}$ and $\mathrm{S}\left({ }^{1} D\right)+\mathrm{C}_{2} \mathrm{H}_{4}$, two viable routes for formation of $\mathrm{C}-\mathrm{S}$ containing molecules. Implications for the formation of prebiotic molecules in several environments will be discussed.

Balucani, N., et al. (2006). Crossed molecular beam reactive scattering: from simple triatomic to multichannel polyatomic reactions. Int. Rev. Phys. Chem., 25: 109-163.

Balucani, N. and Casavecchia, P. (2006). Gas-phase reactions in extraterrestrial environments: laboratory investigations by crossed molecular beams. Orig. Life Evol. Biosph., 36:443-450.

Casavecchia, P. et al. (2001). Crossed beam studies of elementary reactions of $\mathrm{N}$ and $\mathrm{C}$ atoms and CN radicals of importance in combustion. Faraday Discuss., 119: 27-49.

Costes, M., et al. (2006). Crossed-beam studies on the dynamics of the $\mathrm{C}+\mathrm{C}_{2} \mathrm{H}_{2}$ interstellar reaction leading to linear and cyclic $\mathrm{C}_{3} \mathrm{H}+\mathrm{H}$ and $\mathrm{C}_{3}+\mathrm{H}_{2}$. Faraday Discuss., 133: $157-176$.

Leonori, F., et al. (2008). Crossed molecular beam study of gas phase reactions relevant to the chemistry of planetary atmospheres: The case of $\mathrm{C}_{2}+\mathrm{C}_{2} \mathrm{H}_{2}$. Planet. Space Sci., in press, doi:10.1016/j.pss.2008.04.011.

E-mail: nadia.balucani@unipg.it

\section{Prebiotic Synthesis Under Hydrothermal Conditions}

Marie-Paule Bassez

Universite de Strasbourg, IUT Robert Schuman, 72 route du Rhin, 67400 ILLKIRCH France

The fluid compositions of the MAR hydrothermal sites: Rainbow, $36^{\circ} 14^{\prime} \mathrm{N}, 2300 \mathrm{~m}$, Logatchev, $14^{\circ} 45^{\prime} \mathrm{N}, 2,970 \mathrm{~m}$ and Ashadze, $12^{\circ} 58^{\prime} \mathrm{N}, 4,080 \mathrm{~m}$ have been analyzed since 
1997 (Charlou, et al. 2002, Schmidt, et al. 2007, Charlou, et al. 2007, Konn, et al. 2007). They show a great amount of $\mathrm{H}_{2}, \mathrm{CO}_{2}, \mathrm{CH}_{4}$ and $\mathrm{N}_{2}$, and organic molecules of abiotic origin. They are all located on ultramafic geological environments where serpentinization process occurs.

Considering the physico-chemical characteristics of these hydrothermal sites, considering that water in the hydrothermal fluids is in the supercritical state (Bassez, 1999, 2003) and considering also the geological environment and the conditions of heat, radioactivity and volcanism $3.8 \mathrm{Ga}$ ago, experiments of prebiotic synthesis under hydrothermal conditions are proposed.

When peridotite, the rock of the mantle, is dissolved in seawater at $200^{\circ} \mathrm{C}$ and 500 bar, $25 \mathrm{mmol}$ of $\mathrm{H}_{2}$ are measured after 2,000 h (Seyfried, 2007) amount which corresponds to the $\mathrm{H}_{2}$ content of the Rainbow hydrothermal fluids: $16 \mathrm{mmol} \mathrm{of} \mathrm{H}_{2} / \mathrm{kgw}$ and of Logatchev: $12 \mathrm{mmol} / \mathrm{kgw}$. Released $\mathrm{H}_{2}$ can react with $\mathrm{CO}_{2}$ embedded inside the rock to produce $\mathrm{CH}_{4}$. Consequently, if $\mathrm{N}_{2}$ is added to a mixture of peridotite in seawater, i.e. a mixture of $\mathrm{H}_{2}, \mathrm{CO}_{2}, \mathrm{CH}_{4}$, elevated at high-pressure and high temperature or at HPHT of the supercritical state of water, biological molecules observed in Miller's experiments should be synthesized. An excitation process could come from gamma rays simulating the terrestrial radioactivity or from the products of water radiolysis by gamma rays, such as hydrated electron, $\mathrm{H}^{+}, \mathrm{H}_{2} \mathrm{O}_{2}$ or $\mathrm{O}_{2}$. Instead of peridotite, olivine and pyroxene could be the starting reactants. In-situ Raman spectroscopy could allow analyses of the synthesized products. Homochiral molecules could be obtained since olivine, pyroxene and serpentine have octahedral sites between tetrahedral ones, where small elements $\mathrm{H}, \mathrm{C}, \mathrm{N}, \mathrm{O}$ could insert with a specific spatial orientation. These experiments of hydrothermal synthesis have been described in the proceedings of CNRIUT'08 and in Comptes Rendus Chimie (Bassez, 2008).

Bassez, M.-P. (1999). La structure de l'eau supercritique et l'origine de la vie. In l'Harmattan editions, Science et Technologie, Regards Croises, Paris, France, 583-591.

Bassez, M.-P. (2003). Is high-pressure water the craddle of life? J. Phys.: Condens. Matter, 15:L353-L361.

Bassez, M.-P. (2008). Synthese prebiotique hydrothermale. In CNRIUT'08, Proceedings, 29 may, Lyon, France, 1-8.

Bassez, M.-P. (2008). Prebiotic synthesis under hydrothermal conditions. C. R.. Chimie, Acad. Sciences, Paris, France, submitted on june/5.

Charlou, J. L., Donval, J. P., Fouquet, Y., Jean-Baptiste, P., Holm, N. (2002). Geochemistry of high $\mathrm{H}_{2}$ and $\mathrm{CH}_{4}$ vent fluids issuing from ultramafic rocks at the Rainbow hydrothermal field. Chemical Geology, 191:345-359.

Charlou, J., Donval, J., Konn, C., Birot, D., Sudarikov, S., Jean-Baptiste, P., Fouquet, Y. (2007). High hydrogen and abiotic hydrocarbons from new ultramafic hydrothermal sites between $12^{\circ} \mathrm{N}$ and $15^{\circ} \mathrm{N}$ on the Mid-Atlantic

Ridge. Results of the Serpentine cruise (march 2007). Proceedings.

Konn, C., Charlou, J. L., Donval, J. P., Holm, N. G., Dehairs, F., Bouillon, S. (2007). Organics in hydrothermal fluids from 4 ultramafic-hosted vents of the MAR. Results from the Serpentine cruise. Geophysical Research Abstr 2008, 10-EGU2008-A-01497.

Schmidt, K., Koschinsky, A., Garbe-Schönberg, D., M. de Carvalho, L., Seifert, R. (2007). Geochemistry of hydrothermal fluids from the ultramafic-hosted Logatchev hydrothermal field, $15^{\circ} \mathrm{N}$ on the Mid-Atlantic Ridge. Chemical Geology, 242 (1-2): $1-21$. 
Seyfried, W.E. Jr., Foutoukos D.I., Fu Qi (2007). Redox evolution and mass transfer during serpentinization: an experimental and theoretical study at $200^{\circ} \mathrm{C}, 500$ bar with implications for ultramafic-hosted hydrothermal systems at Mid-Ocean Ridges. Geochemica et Cosmochimica Acta, 3872-3886.

E-mail: marie-paule.bassez@urs.u-strasbg.fr

URL: http://www-iut-schuman.u-strasbg.fr/chemphys/mpb

\section{Detection of AIB in Antarctic Ice Samples: Implications for Exogenous Delivery of Prebiotic Organic Compounds}

Oliver Botta ${ }^{1}$, Daniel P. Glavin ${ }^{2}$, Jason P. Dworkin ${ }^{2}$, Graciela Matrajt ${ }^{3}$, Ralph P. Harvey ${ }^{4}$

${ }^{1}$ International Space Science Institute, Hallerstrasse 6, 3012 Bern, Switzerland; ${ }^{2}$ NASA Goddard Space Flight Center, Greenbelt, MD 20771, USA; ${ }^{3}$ Department of Astronomy, University of Washington, Seattle, WA 98195, USA; ${ }^{4}$ Department of Geology, Case Western Reserve University, Cleveland, OH 44106, USA.

Antarctica is the major source of meteorites today. Meteorites are collected at Stranding Surfaces where they accumulate over long periods of time (up to 10,000 years, Harvey, 2003). Due to the long residence time in the ice, exchange of organic matter between the two sources can potentially lead to either a) leaching of organic compounds from the meteorite, and/or b) introduction of terrestrial contamination into the meteorites. This becomes particularly critical when the organic content of the meteorites is low, such as in Martian meteorites, which in turn could compromise the search for traces of molecular biosignatures in these samples. In a previous study we compared the distribution and abundance of amino acids and Polycyclic Aromatic Hydrocarbons (PAHs) in meteorites and their associated ice samples collected at LaPaz icefield, Antarctica in 2003/2004 (Botta et al., in press). Very low concentrations of PAHs in the ice were found, but some of the samples, including an ice sample that did not have a meteorite near it, contained, among other amino acids, a-aminoisobutyric acid (AIB), an abundant non-protein amino acid of extraterrestrial origin. This finding has led to the hypothesis that amino acids could have been leached out of microscopic meteorite samples during the extraction procedure or during the residence time of these particles in the ice. A new set of ice samples, collected in 2006/2007 from North Grave, Antarctica, was analyzed following a modified sample preparation to remove microscopic particular matter, including Antarctic micrometeorites (AMMs), prior to ice meltwater evaporation and focusing on the analysis of the amino acid composition in the residue using Liquid Chromatography with UV Fluorescence and Timeof-Flight Mass Spectrometry (LC-FD/ToF-MS). Two meteorites, a CR2 and a CV3, were collected on top of the ice samples. The ice sample collected with the CR2 meteorite contained AIB above the analytical limit of detection (LoD). No AIB was detected in any other sample above LoD. We were unable to identify any AMMs on the $0.2 \mathrm{~mm}$ filters by visual optical microscope inspection. The corresponding meteorite samples contained only b-alanine and g-amino- $n$-butyric acid above LoD; no AIB was detected in the meteorites. The combined results of both campaigns suggest that contamination of Antarctic meteorites from surrounding ice with either amino acids or PAHs is negligible. The source of AIB in some of the ice samples from LaPaz and North Graves is likely AMMs. Together with preliminary results from the analysis of a set of eight Antarctic meteorites (CM2, CM1, CM1/2 and CR), which display a wide variability of amino acids in 
concentrations up to ten times higher than those found in the Murchison meteorite (Martins et al., 2007), these findings strongly support the notion that exogenous delivery of organic matter to the early Earth contributed significantly to the inventory of organic compounds on the early Earth and probably crucial for the origin of life.

Botta, O. et al., (2008). Polycyclic aromatic hydrocarbons and amino acids in meteorites and ice samples from LaPaz icefield, Antarctica. Meteoritics and Planetary Science, in press. Harvey, R. P. (2003). The origin and significance of Antarctic meteorites. Chemie der Erde, 63: 93-147.

Martins, Z. et al. (2007). Indigenous amino acids in primitive CR meteorites. Meteoritics and Planetary Science, 42: 2125-2136.

Matrajt, G. et al. (2004). Concentration and variability of the AIB amino acid in polar micrometeorites: Implications for the exogenous delivery of amino acids to the primitive Earth. Meteoritics and Planetary Science, 39: 1849-1858.

E-mail: botta@issibern.ch

\title{
Monte Carlo Simulation of Water and Methanol on Grain Surfaces
}

\author{
Sonali Chakrabarti ${ }^{1,2}$, Sandip K. Chakrabarti ${ }^{3,1}$, A. Das ${ }^{2}$, K. Acharyya ${ }^{3}$
}

${ }^{1}$ Maharaja Manindra Chandra College, Kolkata; ${ }^{2}$ Indian Centre for Space Physics, Kolkata; ${ }^{3}$ S. N. Bose National Centre for Basic Sciences, Salt Lake, Kolkata

We use a Monte Carlo simulation to follow the chemical processes occurring on the grain surface. We carry out the simulations on the Olivine grains of different sizes, temperatures, gas phase abundances and different reaction mechanisms. We consider $\mathrm{H}, \mathrm{O}$ and $\mathrm{CO}$ as the accreting species from the gas phase and allow ten chemical reactions among them on the grains. We find that the formation rate of various molecules is strongly dependent on the binding energies. When the binding energies are high, it is very difficult to produce significant amount of the molecular species. Instead, the grain is found to be full of atomic species. The production rates are found to depend on the number density in the gas phase. When the density is high, the production of various molecules on the grains is small as grain sites are quickly filled up by atomic species. If both the Eley-Rideal and Langmuir-Hinselwood mechanisms are considered, then the production rates are maximum and the grains are filled up relatively faster. Thus, if allowed, the Eley-Rideal mechanism can also play a major role and more so when the grain is full of immobile species. We show that the concept of the effective grain surface area which we introduced in our earlier work, plays a significant role in grain chemistry.

E-mail: sonali@csp.res.in

\section{Evolution of Pre-biotic Molecules during Collapse of Interstellar Clouds}

Sandip K. Chakrabarti, S. N. Bose

National Centre for Basic Sciences, JD Block, Salt Lake, Kolkata 700098 and Indian Centre for Space Physics, Kolkata

Discovery of amino acids in meteorites suggest that many of the complex pre-biotic molecules could indeed be formed during the collapse of the interstellar clouds before the 
actual star formation took place. We carry out such studies using complete grain and gas chemistry. We use rate equation method, master equation method as well as the MonteCarlo method to show evolution of lighter molecules in the grain phase and subsequently desorb them to the gas phase and evolve them to produce more complex molecules. Our results generally match with observations for lighter molecules. However, for complex molecules the result is not so conclusive. We believe that this is due to our poor knowledge of the reaction pathways and the reaction cross-section for complex molecules.

E-mail: chakraba@bose.res.in

\section{Optical Emission Spectroscopy of High-Power Laser-Induced Dielectric Breakdown in Molecular Gases and Their Mixtures: Investigating Early Stages of Plasma Chemical Action in Planetary Atmospheres}

Jaroslav Cihelka ${ }^{1,2}$, Irena Matulková ${ }^{1}$, Kristéna Sovová ${ }^{1}$, Michal Kamas ${ }^{1}$, Petr Kubelík ${ }^{1,2}$, Martin Ferus ${ }^{1,2}$, Libor Juha ${ }^{2}$, Svatopluk Civiš ${ }^{1}$

${ }^{1}$ J. Heyrovsky Institute of Physical Chemistry, Academy of Sciences of the Czech Republic, v.v.i., Dolejškova 3, 18223 Prague 8, Czech Republic; ${ }^{2}$ Institute of Physics, Academy of Sciences of the Czech Republic, v.v.i., Na Slovance 2, 18223 Prague 8, Czech Republic

The main goal of this work was simulation of potential high energy processes in early Earth's atmosphere (as meteorite impact, lightning), which could lead to more complex compounds generated from simple molecular gases (Babánková, Cihelka et al. 2006). Large-scale plasma was created in molecular gases $\left(\mathrm{CH}_{4}, \mathrm{~N}_{2}, \mathrm{D}_{2} \mathrm{O}\right)$ and their mixtures by high-power laserinduced dielectric breakdown (LIDB). Compositions of the mixtures used are those suggested for the early Earth's atmosphere (Babánková et al. 2006). Time-integrated as well as timeresolved optical emission spectra emitted from the laser spark have been measured and analyzed. The spectra of the plasma generated in the $\mathrm{CH}_{4}, \mathrm{~N}_{2}$ and $\mathrm{D}_{2} \mathrm{O}$ containing mixtures are dominated by emission of $\mathrm{C}_{2}$ and $\mathrm{CN}$ radicals. These species are precursors of stable products as acetylene and hydrogen cyanide. Occurrence of both species was confirmed in irradiated gaseous mixture by FTIR spectroscopy and gas chromatography (Civiš et al. in press). Gas chromatographic signals also indicate a presence of other complex species in a low concentration. The GC peaks were assigned to ethene, propene, propine and allene.

\section{Acknowledgements}

This work financially supported by Grant Agency of the Czech Republic (grant No. 203/06/ 1278) and the Czech Ministry of Education (grants LC510, LC528, and LA08024).

Babánková D., Civiš S., Juha L., Bittner M., Cihelka J., Pfeifer M., Skála J., Bartnik A., Fiedorowicz H, Mikolajczyk J., Šedivcová T. (2006). Optical and x-ray emission spectroscopy of high-power laser-induced dielectric breakdown in molecular gases and their mixtures. Journal of Physical Chemistry A, 110:12113-12120.

Babánková D., Civiš S., Juha L. (2006). Chemical consequencies of laser-induced breakdown in molecular gases. Progress in Quantum Electronics, 30:75-88.

Civiš S., Babánková D., Cihelka J., Sazama P., Juha L. (in press). Spectroscopic investigation of high-power laser-induced dielectric breakdown in gas mixtures containing carbon monooxide. To appear in the Journal of Physical Chemistry A

E-mail: jaroslav.cihelka@jh-inst.cas.cz 


\title{
Surfaces as Concentration Agents in Chemical Evolution
}

\author{
María Colín-García, Alicia Negrón-Mendoza, Sergio Ramos-Bernal
}

On Primitive Earth, concentration of many organic molecules on the oceans may be low, between 0.003 and $0.03 \mathrm{M}$ (Miller \& Orgel 1974), some reactions could have taken place under these conditions, but many others may not. So, the existence of concentration mechanisms should be crucial. Different solid surfaces have been proposed, mainly minerals, for supporting compounds. The most important ones are silicates, carbonates, sulfates and clays. Clays are important because of their wide spatial and temporal distribution and their strong affinity for organic compounds (Ponnamperuma et al. 1982). Clays could have played the role as concentration, catalyst and protective agents for prebiotic molecules against destructive energy sources (Bernal 1951). Furthermore, silicates are key component of Earth, interstellar dust, asteroids, and comets. In this work, different surfaces were chosen in order to explore their capacity to retain hydrogen cyanide $(\mathrm{HCN})$. $\mathrm{HCN}$ is widely recognized as a key molecule in prebiotic studies, because it is present in the ISM (Irvine 1998, Boonman et al. 2001), comets (Ip et al. 1990, Magee-Sauer et al. 1999, Gerakines et al. 2004), and in the atmosphere of different satellites. It is precursor of molecules such as: carboxylic acids, amino acids and purine and pyrimidine bases (Oró \& Lazcano-Araujo 1981). However, HCN is very volatile and its polymerization capacity is low at diluted conditions; so, concentration mechanism should have been fundamental for it.

Aliquots of a HCN solution were mixed up with different surfaces such as: silica gel, sodium montmorillonite, calcium montmorillonite, kaolinite, attapulgite and hectorite, to explore the capacity of all these to retain HCN. Results show that clays are better adsorbents that amorphous silicates. In silica gel just a fraction of $\mathrm{HCN}$ is adsorbed. $\mathrm{HCN}$ is partially adsorbed in sodium montmorillonite at natural $\mathrm{pH}$ conditions $(\mathrm{pH}$ 6). At acidic conditions all the hydrogen cyanide is adsorbed; on the other hand, when $\mathrm{pH}$ is basic no adsorption is observed. This suggest that adsorption of $\mathrm{HCN}$ in sodium montmorillonite is mainly by cationic interchange. When the same clay, but with a different cation in the interlamellar channel (calcium), is tested the same behavior is observed. A small amount of $\mathrm{HCN}$ is taken by kaolinite, and when $\mathrm{pH}$ is acidified a smaller fraction is retained due to clay starts to decompose. The adsorption of HCN in hectorite and attapulgite is differential. In the first case, just a very small amount is adsorbed, in the other, all is taken. Among clay minerals those with a high cationic interchange capacity or high superficial area are better adsorbents for HCN. Thus, we can propose clays as very good substrates to retain and concentrate this type of molecule.

Bernal, J. D. (1951). The Physical Basis of Life. Rutledge and Keegan Paul, London.

Boonman, A. M. S., Stark, R., van der Tak, F. F. S., van Dishoek, E. F., van der Wal, P. B., Shäfer, F., de Lange, G., and Laauwen, W. M. (2001). Highly Abundant HCN in the Inner Hot Envelope of GL 2591: Probing the Birth of a Hot Core? Astrophysics Journal, 553: L63-L67.

Gerakines, P. A., Moore, M. H., and Hudson, R. L. (2004). Ultraviolet Photolysis and Proton Irradiation of Astrophysical Ice Analogs Containing Hydrogen Cyanide. Icarus, 170: 202-213.

Irvine, W. M. (1998). Extraterrestrial Organic Matter: A Review. Origins of Life and Evolution of the Biosphere, 28: 365-383.

Ip, W. H., Balsiger, H., Geiss, J., Goldstein, B. E., Kettmann, G., Lazarus, A. J., Meier, A., Rosenbauer, H., and Schelley, E. (1990). Giotto ISM Measurements of the Production Rate of Hydrogen Cyanide in the Coma of Comet Halley. Annales Geophysicae, 8: 319-325. 
Magee-Sauer K., Mumma, M. J., DiSanti, M. A., Russo, N. D., and Rettig, T. W. (1999). Infrared Spectroscopy of the $v 3$ Band of Hydrogen Cyanide in Comet C/1995 O1 HaleBopp. Icarus, 142: 498-598.

Miller, S. and Orgel, L. (1974). The Origins of Life on the Earth. Prentice Hall, Inc., New Jersey. Oró, J. and Lazcano-Araujo, A. (1981). The Role of HCN and its Derivatives in Prebiotic Evolution. In Vennesland, B., Conn, E. E., Knowles, C. J., Westley, J. and Wissing, F., editors, Cyanide in Biology, pages 517-541. Academic Press, London.

Ponnamperuma, C., Shimoyama, A., and Friebele, E. (1982). Clay and the Origin of Life. Origins of Life, 12: 9-40.

E-mail: mcolin@nucleares.unam.mx

\title{
Analysis of Sugar Derivatives in Carbonaceous Meteorites
}

\author{
George Cooper, Minakshi Sant, Alanna O’leary, Cynthia Asiyo
}

\section{NASA-Ames Research Center, Space Science Division Moffett Field, CA 94035}

Carbonaceous meteorites contain a diverse suite of soluble organic compounds. These compounds were delivered to the early Earth in asteroids (and possibly comets) and therefore may have played an important role in the origin and/or evolution of life. Among the classes of organic compounds found in carbonaceous meteorites are amino acids, amides, and sugar derivatives. Our current research involves the study of the enantiomeric ( $\mathrm{d} / \mathrm{l}$ mirror image) and isotopic properties of meteoritic sugar acids (Cooper et al., 2001). In life as we know it, only one of two possible enantiomers are used in proteins ( 1 amino acids) and nucleic acids (d sugars), these polymers are homochiral. In a natural (non-biological) process, such as that expected to have operated on the parentbody of the meteorites, equal amounts of $\mathrm{d}$ and 1 enantiomers should be synthesized because (as far as we know) enantiomers have equal energies of formation. Equal d/ 1 abundances are the norm for the vast majority of chiral meteoritic compounds, however, some meteorite amino acids contain enantiomeric excesses (Pizzarello et al., 2006). Due to their structural relationships to organic compounds used in biochemistry, the analysis of enantiomer ratios of meteoritic compounds may have implications for understanding the origins of homochirality on Earth.

In the case of enantiomeric analysis of meteorite sugar acids we have successfully separated several enantiomer pairs and analyses of the Murchison and Murray meteorites show that in the majority of individual acids there are equal abundances of enantiomers, however there appear to be exceptions. There are indications of enantiomeric excesses in four and fivecarbon sugar acids that are not easily explained by microbial action. In addition, in each series of four through six-carbon sugar acids, rare as well as common compounds are present: an indicator of an abiotic synthesis process. The smallest of the meteorite sugar acids, glyceric, is also the most widely distributed on Earth in biological systems and would appear to be the most likely to contaminate meteorite samples. However meteoritic glyceric is consistently racemic and a ${ }^{13} \mathrm{C}$ analysis shows it to be of extraterrestrial origin. Results of further enantiomeric and isotopic analyses as well as studies on microorganisms will be presented.

Cooper, G., Kimmich, N., Belisle, W., Sarinana, J., Brabham, K., and Garrel, L. (2001). Carbonaceous meteorites as a source of sugar-related organic compounds for the Early Earth. Nature, 414: 879-883. 
Pizzarello, S., Cooper, G. W., and Flynn, G. J. (2006). The Nature and Distribution of the Organic Material in Carbonaceous Chondrites and Interplanetary Dust Particles in Meteorites and the Early Solar System II, pp. 625-651. D. S. Lauretta and H. Y. McSween Jr. (eds.), University of Arizona Press, Tucson.

E-mail: gcooper@mail.arc.nasa.gov

\title{
Dramatic Alteration of the Thermal Behavior of Glycine by Ca-Montmorillonite
}

\author{
Punam Dalai, Henry Strasdeit
}

Department of Bioinorganic Chemistry, Institute of Chemistry, University of Hohenheim, 70599 Stuttgart, Germany

An important but less studied aspect of chemical evolution is the interaction of organic matter with its inorganic environment. As an example, clays are among the prebiotically most important minerals, particularly because of (a) the ability to bind various organic molecules in their interlayer spaces, (b) large surface-to-volume ratios, and (c) their suitability for absorption and catalytic processes (e. g. Anderson and Banin, 1975). Clays might have played a central role in molecular evolution on the early Earth (Brack, 2006; Bujdák and Rode, 1995; Cairns-Smith and Hartman, 1988; Ponnamperuma et al., 1982). The polymerization of glycine up to the tetrapeptide was achieved on bentonite in a fluctuating environment at $80^{\circ} \mathrm{C}$ (Lahav et al., 1978). In our experiments, we found that at temperatures around $200^{\circ} \mathrm{C}$ glycine loaded Ca-montmorillonite showed two contrasting behaviors: it catalyzed peptide bond formation but also protected the amino acid against irreversible condensation. In a prebiotic environment, such high temperatures may have occurred in active volcanic regions.

A typical experiment was as follows. The Ca-montmorillonite SAz-1 obtained from the Clay Minerals Society was used. A sample, which had a particle size of $\leq 2 \mu \mathrm{m}$, was suspended in $0.5 \mathrm{~mol} / \mathrm{L}$ glycine solution. The glycine loaded clay was isolated and dried. Then it was kept at $200^{\circ} \mathrm{C}$ in a nitrogen atmosphere for $48 \mathrm{~h}$. Afterwards, most of the residue was again suspended in water. The water was removed by evaporation, the clay was dried, and the heating repeated. Four wetting-drying-heating cycles were performed. After each thermolysis, samples of the residue were extracted with $\mathrm{H}_{2} \mathrm{O}, \mathrm{D}_{2} \mathrm{O}$ and dilute trifluoroacetic acid, respectively, and subsequently analysed by HPLC, NMR and MALDITOF-MS. Besides large amounts of unreacted amino acid, the cyclic diglycine (diketopiperazine) and linear peptides up to the hexapeptide were detected. No chain elongation was observed in the course of the wetting-drying-heating cycles.

When glycine is kept at $200^{\circ} \mathrm{C}$ in a nitrogen atmosphere in the absence of montmorillonite, small amounts of the cyclic dipeptide and a deep black residue (termed as "thermo-melanoid") are obtained. The thermo-melanoid is water-insoluble. Its chemical nature is unknown but our data indicate that its formation may be due to unconventional condensation reactions between peptide intermediates. Clearly, Ca-montmorillonite protects glycine from being irreversibly transformed into the thermo-melanoid and thus alters the thermal behavior of glycine fundamentally.

\section{Acknowledgements}

Financial support from the Deutsche Forschungsgemeinschaft is gratefully acknowledged. 
Anderson, D. M. and Banin, A. (1975). Soil and water and its relation to the origin of life. Orig. Life, 6:23-36.

Brack, A. (2006). Clay minerals and the origin of life. In Bergaya, F., Theng, B. K. G. and Lagaly, G., editors, Handbook of Clay Science, pages 379-391. Elsevier, Amsterdam.

Bujdák, J. and Rode, B. M. (1995). Clay and their possible role in prebiotic peptide bond synthesis. Geol. Carpathica, Ser. Clays, 4:37-48.

Cairns-Smith, A.G. and Hartman, H. (1988). Clay Minerals and the Origin of Life. Cambridge University Press, UK.

Lahav, N., White, D., and Chang, S. (1978). Peptide formation in the prebiotic era: thermal condensation of glycine in fluctuating clay environments. Science, 201: 67-69.

Ponnamperuma, C., Shimoyama, A., and Friebele, E. (1982). Clay and the origin of life. Orig. Life Evol. Biosph., 12: 9-40.

E-mail: pdalai@uni-hohenheim.de

\section{V.U.V. Irradiation of Interstellar Ice Analogs: A Potential Source for Prebiotic Molecules in Planetary Systems}

\section{G. Danger ${ }^{1}$, P. de Marcellus ${ }^{1}$, Z. Djouadi ${ }^{1}$, J.B. Bossa ${ }^{2}$, T. Chiavassa ${ }^{2}$, L.d'Hendecourt $^{1 *}$}

${ }^{1}$ Astrochimie et Origines, Institut d'Astrophysique Spatiale, Orsay, France. ${ }^{2}$ Spectromatries et Dynamique Moléculaire, Physique des Interactions Ioniques et Moléculaires, Université de Provence, Marseille, France

The study of astrophysical chemistry is an important task to understand matter evolutions in the Universe and notably evolution pathways from abiotic chemistry in the interstellar medium (MIS) to prebiotic chemistry in planetary systems. In the dense interstellar medium, the major part of light elements $(\mathrm{O}, \mathrm{C}, \mathrm{N})$ is adsorbed on interstellar grains. From a schematic point of view, these grains are formed with different layers which included ices of volatile compounds which surround residue of refractory carbon and a nucleus consisting of silicate compounds. The physical and chemical evolution of these grains is followed by parent body's aggregation, the first step towards planetary formation.

From experimental simulations of astrophysical environments in the laboratory, and particularly conditions of molecular ices formation, our aim is to understand the chemical evolution of these ices in order to retrace the chemical evolution toward complex molecule formation in the ISM (e.g. pathways for amino acid synthesis or their precursors). The first results obtained after ice analogs (e.g. including $\mathrm{H}_{2} \mathrm{O}, \mathrm{CO}, \mathrm{NH}_{3}, \mathrm{CH}_{3} \mathrm{OH}$...) irradiation have shown the formation of radicals and more complex molecules by infrared in situ analysis. The first step is thus to compare these data with astronomical observations in order to identify the importance of photochemical processes in astrophysical environments. After sample heating, radicals and molecules can rearrange to form a residue which includes complex organic molecules such as amino acids, detected after hydrolysis treatment of the samples (Bernstein et al., 2002; Muñoz-Caro et al., 2002; Nuevo et al., 2008). Without this treatment only very few amounts of amino acids are detected (Nuevo et al., 2008). This observation leads to the hypothesis that amino acids could be included in a complex structure which, after degradation, releases them during hydrolysis. Another possibility is that amino acids could come from precursors such as nitriles (Elesila et al, 2007). The last hypothesis is corroborated by the recent aminoacetonitrile detection in the ISM gas phase (Belloche et al., 2008). We present here our investigation of aminoacetonitrile pathway formation, a potential precursor of glycine in the ISM. 
Beside the ice irradiation alone, we also investigate the possible catalytic effect of a silicate surface during irradiation and estimate the influence on the molecule abundance and variety (Brucato et al., 2006; Hill et al., 2003). We present our first results on the photolysis of ices on realistic (e.g. interstellar) silicate surfaces and discuss the validity of our experimental approach for the production and study of organic residues in astrophysical environments.

Belloche, A., Menten, K. M., Comito, C., Müller, H. S. P., Schilke, P., Ott, J., Thorwirth, S., Hieret, C., (2008) Detection of amino acetonitrile in Sgr B2(N), Astron. Astrophys., 482:179-196.

Bernstein, M. P., Dworkin, J. P., Sandford, S. A., Cooper, G. W., Allamandola, L. J., (2002) Racemic amino acids from the ultraviolet photolysis of interstellar ice analogues, Nature, 416:401-403.

Brucato, J. R., Strazzulla, G., Baratta, G. A., Rotundi, A., Colangeli, L., (2006) Cryogenic synthesis of molecules of astrobiological interest: catalytic role of cosmic dust analoques, Origins Life Evol. Biosphere, 36:451-457.

Elsila, J. E., Dworkin, J. P., Bernstein, M. P., Martin, M. P., Sandford, S. A., (2007) Mechanisms of amino acid formation in interstellar ice analogs, Astrophys. J., 660:911918.

Hill, H. G. M., Nuth, J. A., (2003) The Catalytic Potential of Cosmic Dust: Implications for Prebiotic Chemistry in the Solar Nebula and Other Protoplanetary Systems, Astrobiology, 3:291-304.

Muñoz-Caro, G. M., Meierhenrich, U. J., Schutte, W. A., Barbier, B., Arcones Segovia, A., Rosenbauer, H., Thiemann, W. H.-P., Brack, A., Greenberg, J. M., (2002) Amino acids from ultraviolet irradiation of interstellar ice analogues, Nature, 416:403-406.

Nuevo, M., Auger, G., Blanot, D., D’Hendecourt, L., (2008) A detailed analysis of the amino acids produced after the vacuum UV irradiation of ice analogs, Origins Life Evol. Biosphere, 38:37-56.

E-mail: dangergregoire@yahoo.fr

\section{The Role of Ionizing Radiation on Simple Prebiotic Mixtures, a Comparison with UV Irradiation}

Daniele Dondi ${ }^{1}$, Daniele Merli ${ }^{1}$, Luca Pretali $^{2}$, Antonio Faucitano ${ }^{1}$, Armando Buttafava ${ }^{1}$

${ }^{1}$ Department of General Chemistry, University of Pavia, via Taramelli 12, 27100 Pavia, Italy; ${ }^{2}$ Department of Organic Chemistry, University of Pavia, via Taramelli 10, 27100 Pavia, Italy

Prebiotic chemical evolution encompass the sequence of events on the primitive Earth that led to the formation of complex organic compounds from simple organic and inorganic molecules (Oparin-Haldane hypothesis). According to this hypothesis, the synthesis of organic compounds on the prebiotic Earth, their transformation in more complex molecules and the generation of replicating systems were important steps which led to the appearance of life. Starting from the very first prebiotic-chemistry experiment, different energy sources were used to sustain the synthesis of organic molecules of biological interest starting from simple compounds. It is generally admitted that ionizing radiation was one of energy sources in the prebiotic environment, particularly for the abundance of radionuclides in the Earth's crust. However, little attention has been paid to it (see, for example, Ramos-Bernal 
and Negron-Mendoza, 1998; Draganic et al., 1977; Albarran et al., 1988; Kolomnikov et al., 1982).

We decide to explore the chemistry of model simple prebiotic mixtures with the help of modern analytical techniques. Binary and ternary water mixtures of simple organic compounds (alcohols, ketones, ammonia and amines) were irradiated by a Co-60 gamma source (500-800 KGy total dose) and products were analyzed by GC-MS technique.

Relative concentration were chosen to maintain constant the $\mathrm{C}: \mathrm{H}: \mathrm{N}: \mathrm{O}$ ratio.

As products we also found hexamethylenetetramine, pyrroles, pyrazines and pyrimidines.

In the course of the presentation will be discussed possible reaction mechanisms leading to the formation of products observed and a comparison between gamma irradiation and UV irradiation (Dondi et al., 2007) of the tested mixtures.

Albarran, G., Negron-Mendoza, A. Trevino, C. and Torres, J. L. (1988) Role of ionizing radiation in chemical evolution studies. Radiat. Phys. Chem., 31:821-823.

Dondi, D., Merli, D., Pretali, L., Fagnoni, M., Albini, A., and Serpone, N. (2007) Prebiotic chemistry: chemical evolution of organics on the primitive Earth under simulated prebiotic conditions. Photochem Photobiol Sci. 6:1210-1217.

Draganic, Z., Draganic, I., Shimoyama, A. and Ponnamperuma, C. (1977) Evidence for amino acids in hydrolyzates of compounds formed by ionizing radiations. I. Aqueous solutions of hydrogen cyanide, ammonium cyanide, and sodium cyanide. Origins of Life 8:371-376.

Kolomnikov, I. S., Lysyak, T. V., Konash, E. P., Kalyazin, E. P., Rudnev, A. V. and Kharitonov, Y. Y. (1982) Formation of organic products from metal carbonates and water in the presence of ionizing radiation. Doklady Akademii Nauk SSSR 265:912-913.

Ramos-Bernal, S. and Negron-Mendoza, A. (1998). Surface chemical reactions during the irradiation of solids. Prebiotic relevance. Viva Origino, 26:169-175.

E-mail: dondi@unipv.it

\section{Exogenous Delivery and Molecular Evolution: Peptides Based on C-methylated $\alpha$-Amino Acids as Asymmetric Catalysts in the Syntheses of Simple Sugars}

Fernando Formaggio ${ }^{1}$, Alessandro Moretto ${ }^{1}$, Claudio Toniolo ${ }^{1}$, Quirinus B. Broxterman ${ }^{2}$, Arthur L. Weber ${ }^{3}$, Sandra Pizzarello ${ }^{4}$

${ }^{1}$ Department of Chemistry, University of Padova, 35131 Padova, Italy; ${ }^{2}$ DSM Pharmaceutical Products, 6160 MD Geleen, The Netherlands; ${ }^{3}$ SETI Institute, Ames Research Center, Moffet Field, CA 94035-1000, USA; ${ }^{4}$ Department of Chemistry and Biochemistry, Arizona State University, Tempe, AZ 85018-1604, USA.

It has been shown that chiral amino acids, as well as their dipeptides, may catalyze the asymmetric condensation of glycolaldehyde in water (Pizzarello and Weber, 2004; Weber and Pizzarello, 2006). On the basis of the particularly large erythrose enantiomeric excesses (ee) obtained when utilizing the chiral L-Val-L-Val catalyst and given the possibility of an abundant delivery of other types of amino acids to the early Earth, we have studied the catalytic effect on this synthesis of the peptides based on $\mathrm{C}^{\alpha}$-methylated $\alpha$-amino acids, such as isovaline $\left(\mathrm{C}^{\alpha}\right.$-methyl, $\mathrm{C}^{\alpha}$-aminobutyric acid) and $\mathrm{C}^{\alpha}$-methylvaline that are abundant in meteorites.

By exploiting the chemo-enzymatic synthesis developed by DSM Pharmaceutical Products (Sonke et al., 1999), we prepared enantiomerically pure isovaline and $\mathrm{C}^{\alpha}$ methylvaline in large amounts. The corresponding racemic $\alpha$-amino amides, synthe- 
sized by partial Strecker synthesis, were enzymatically resolved with appropriate $\alpha$ amino amidases. Then, homo-peptides (di- and tetra-) from the sterically hindered isovaline and $\mathrm{C}^{\alpha}$-methylvaline were synthesized step-by-step in solution. The highly effective EDC/HOAt or acyl fluoride C-activation procedures were employed in peptide bond formation.

Results of the catalysis experiments showed the all $\mathrm{C}^{\alpha}$-methylated peptides exhibit significant chiral influence on the synthesis of tetroses and mimic the effect of the L-Val-LVal catalyst in having a larger erythrose ee than threose ee, as well as in their configuration relationship with the sugars (the product erythrose acquires ee of configuration opposite to that of the catalyst in case of peptides, while it is the same for amino acids). Interestingly, the largest ee ( $45 \%$ for erythrose) was obtained with the homo-tetrapeptide of isovaline under mild conditions (sodium acetate buffer, $\mathrm{pH} 5.4,25^{\circ} \mathrm{C}, 18 \mathrm{~h}$ ). The homo-dipeptides of both isovaline and $\mathrm{C}^{\alpha}$-methylvaline also produced a significant ee ( $41 \%$ for erythrose) that appears to increase with time.

Because $C^{\alpha}$-methylated amino acids are non-racemic in meteorites, do not racemize in aqueous environments, and are known to be $\left(3_{10}\right)$-helix (Toniolo and Benedetti, 1991) formers in peptides with as few as four residues (Toniolo et al., 2001), these results suggest that meteoritic, $\mathrm{C}^{\alpha}$-methylated, $\alpha$-amino acids may have contributed to molecular evolution upon delivery to the early Earth by catalytically transferring their asymmetry to other prebiotic molecules.

Pizzarello, S., Weber, A. (2004). Meteoritic amino acids as asymmetric catalysts. Science, 303:1151.

Sonke, T., Kaptein, B., Boesten, W. H. J., Broxterman, Q. B., Schoemaker, H. E., Kamphuis, J., Formaggio, F., Toniolo, C., Rutjes, F. P. J. T. (1999). In Patel, R. N., editor, Stereoselective Biocatalysis, pages 23-58. Dekker, New York, NY.

Toniolo, C., Benedetti, E. (1991) The polypeptide 3 10-helix. Trends Biochem. Sci., 16:350353.

Toniolo, C., Crisma, M., Formaggio, F., Peggion, C. (2001). Control of peptide conformation by the Thorpe-Ingold effect ( $\mathrm{C}^{\alpha}$-tetrasubstitution). Biopolymers (Pept. Sci.), 60:396-419.

Weber, A., Pizzarello, S. (2006). The peptide catalyzed stereospecific synthesis of tetroses: a possible model for prebiotic molecular evolution. Proc. Natl. Acad. Sci. USA, 103:12713-12717.

E-mail: fernando.formaggio@unipd.it

\title{
Chemical Evolution: From Amino Acids to Oligopyrroles
}

\author{
Stefan Fox, Henry Strasdeit
}

Department of Bioinorganic Chemistry, Institute of Chemistry, University of Hohenheim,70599 Stuttgart, Germany

It is widely accepted that on the early Earth amino acids from endogenous (e. g. MillerUrey chemistry) and/or exogenous sources (e. g. meteorites) were available (Miller, 1998; Pizzarello, 2004). Amino acids that were dissolved in the primordial ocean remained embedded in a salt crust, when the seawater evaporated at hot volcanic coasts. We have shown that the amino acids coordinate to metal cations in artificial sea salt crusts. Because of this coordination, the amino acids cannot sublime and therefore are forced to undergo chemical reactions at higher temperatures. The thermal transformation of amino acids into 
new compounds could have been an important step in chemical evolution. In previous thermolysis experiments we have simulated this scenario (Fox et al., 2007). Artificial seawater $\left(705 \mathrm{mmol}\right.$ of $\mathrm{NaCl}, 15 \mathrm{mmol}$ of $\mathrm{KCl}, 15 \mathrm{mmol}$ of $\mathrm{CaCl}_{2}$, and $80 \mathrm{mmol}$ of $\mathrm{MgCl}_{2}$ ) that contained amino acids (e. g. rac-alanine) was evaporated at room temperature, and the solid residue was then thermolysed at $350^{\circ} \mathrm{C}$. The volatile products were analyzed by $\mathrm{GC}-$ MS. It was possible to identify several C-alkylated pyrroles, e. g. kryptopyrrole (3-ethyl2,4-dimethylpyrrole). Also large amounts of $\mathrm{HCl}$, resulting from the decomposition of $\mathrm{MgCl}_{2} \cdot 6 \mathrm{H}_{2} \mathrm{O}$ were observed.

It is known that pyrrole, in aqueous $\mathrm{HCl}$ solutions, reacts with formaldehyde to form oligopyrroles (Sobral et al., 2003). We therefore studied the reaction of kryptopyrrole (3 mmol) in a solution of artificial seawater (salt concentration $\sim 4 \%$ ), formaldehyde ( $3 \mathrm{mmol})$ and $\mathrm{HCl}(0.3 \mathrm{mmol})$. Kryptopyrrole, which has only one unsubstituted $\mathrm{C}$ atom, was chosen to keep the number of products low. Formaldehyde is regarded as a prebiotic molecule (e. g. Blair et al., 2008). After $1 \mathrm{~h}$ of reflux, a water insoluble dark green residue was isolated and analyzed by GC-MS. Comparison with an authentic sample proved that the dipyrromethene $\mathbf{1}$ has been formed. Future experiments will focus on (a) prebiotically relevant oxidation reagents such as nitrite and nitrate (Cleaves et al., 2008), (b) the formation of higher oligopyrroles under the conditions of the hot-volcanic-coast scenario, and (c) metal complexes of oligopyrroles.

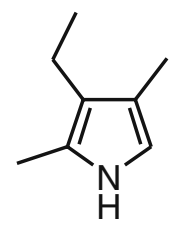

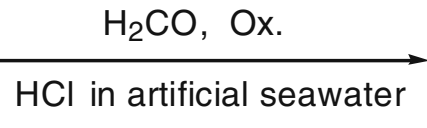

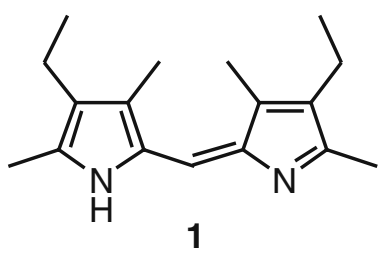

1

The reaction of kryptopyrrole to the corresponding dipyrromethene $\mathbf{1}$ under conditions pertinent to the hot-volcanic-coast scenario.

Blair, S. K., Magnani, L., Brand, J., and Wouterloot, J. G. (2008). Formaldehyde in the far outer galaxy: constraining the outer boundary of the galactic habitable zone. Astrobiology, 8:59-73.

Cleaves, H. J., Chalmers, J. H., Lazcano, A., Miller, S. L., and Bada, J. L. (2008). A reassessment of prebiotic organic synthesis in neutral planetary atmospheres. Orig. Life Evol. Biosph., 38:105-115.

Fox, S., Filippi, J.-J., and Strasdeit, H. (2007). Chemical evolution: pyrroles and pyridines from the amino acid alanine. Int. J. Astrobiol., 6:79; presented at the 7th European Workshop on Astrobiology, Turku, Finland 2007.

Miller, S. L. (1998). The endogenous synthesis of organic compounds. In Brack, A., editor, The Molecular Origins of Life, pages 59-85. Cambridge University Press, Cambridge, UK. Pizzarello, S. (2004). Chemical evolution and meteorites: an update. Orig. Life Evol. Biosph., 34:25-34.

Sobral, A. J. F. N., Rebanda, N. G. C. L., da Silva, M., Lampreia, S. H., Ramos Silva, M., Matos Beja, A., Paixão, J. A., and d'A. Rocha Gonsalves, A. M. (2003). One-step synthesis of dipyrromethanes in water. Tetrahedron Lett., 44:3971-3973.

E-mail: h-strasd@uni-hohenheim.de 


\title{
Synthesis of Organic Molecules During Impacts at Accretion of the Earth and Planets
}

\author{
M. V. Gerasimov ${ }^{1}$, E. N. Safonova ${ }^{1}$, Yu. P. Dikov ${ }^{1,2}$
}

${ }^{1}$ Space Research Institute, RAS, Profsoyuznaya, 84/32, Moscow, 117997, Russia; ${ }^{2}$ Institute of Ore Deposits, Petrography, Mineralogy and Geochemistry, RAS, Staromonetny per.,35, Moscow, 109017, Russia

The earliest stages of the Earth group planets formation was characterized by massive impacts of planetesimals. Impacts of planetesimals provided the output of enormous energy that resulted in the early planetary differentiation and the release of impact-generated atmosphere and water to ocean. Experimental study of impact plume chemistry (Mukhin et al.,1989) showed that the released gas mixture was characterized by the presence of both reduced and oxidized volatile elements components what provided an input of highly nonequilibrium species into ecosystem. Thermal decomposition of petrogenic oxides provides the release of sufficient quantities of molecular oxygen into primordial atmosphere though its availability could be temporal due to rather high sink (Gerasimov, 2002).

An impact of a meteorite into the Earth is generally considered as destructive process for organics because of the action of two main factors: (1) extremely high temperatures and (2) activity of free oxygen in the forming plume. On the other hand impacts can be favorable for organic synthesis providing high-temperature reactions coupled with rapid cooling of agents. The present paper considers the possibility of synthesis of complex organic species from initially inorganic volatile components under conditions of impact-induced plume and discus results of impact-simulation experiments.

Our simulation experiments were performed using standard laser pulse (LP) technique (Gerasimov et al., 1998). Experiments showed rather efficient synthesis of complex organic molecules even at oxidizing conditions. Organic species consisted of alkanes, alkenes, cyclic and polycyclic hydrocarbons, acids, esters, heteroatomic species etc. Most of carbon is bound in soot like structure and highly polymerized hydrocarbons with low solubility in solvents. The amount of formed light organic species is orders of magnitude higher than inferred by gas phase thermodynamic equilibrium (Gerasimov, 2002). We claim for heterogeneous catalysis on the surface of nano-particles of silicates which are condensing everywhere in the spreading cloud.

Impacts of planetesimals provided important processing of the early Earth by producing early impact-generated atmosphere and hydrosphere coupled with the input of nonequilibrium environmental components and synthesis of organic species of various complexities from initially inorganic/organic source elements.

\section{Acknowledgements}

This research was supported by the RAS Program of Basic Research (P-18) and RFBR grant No 07-05-01054.

Gerasimov M.V., et al. (1998) Physics and Chemistry of Impacts. Earth, Moon, and Planets, 80(1-3):209-259.

Gerasimov M.V. (2002) Toxins produced by meteorite impacts and their possible role in a biotic mass extinction. In: Koeberl, C., and MacLeod, K.G., editors, Catastrophic Events and Mass Extinctions: Impacts and Beyond, Boulder, Colorado, Geological Society of America Special Paper 356:705-716. 
Mukhin, L. M. et al. (1989) Origin of precursors of organic molecules during evaporation of meteorites and mafic terrestrial rocks, Nature, 340:46-48.

E-mail: mgerasim@mx.iki.rssi.ru

\title{
Prebiotic Synthesis in Cosmic Environment: In-flight Survival and Formation During Short- and Long-Term Low-Earth Orbiting
}

\author{
Natalia Gontareva, Evgenia Kuzicheva
}

\section{Laboratory of Exobiology, Institute of Cytology}

Abiogenesis - the emergence of life from nonliving physicochemical systems - forms the core of the evolutionary paradigm. Multiple flights at the low earth orbit, the latest results obtained by space missions and laboratory experiments have yielded a new data about structure and composition of cosmic bodies and extraterrestrial environment. All these latest achievements contributed to the belief in possibility of organic compounds synthesis in the outer space environment. Yet the hypothesis of the life origin under strictly natural conditions, especially through interstellar or interplanetary transport, needs more convincing facts as well as the precise analyzing of the data obtained. Experiments conducted on five different Earth-orbiting Russian space missions revealed that cosmic radiation in space both enhanced biochemical synthesis and decayed the biological molecules (nucleosides and peptides) placed on the spacecraft. With long flight durations the degradation reactions always exceeded the synthesis reactions (Kuzicehva and Gontareva 2001). Meanwhile, short-term space flights such as Bion and Foton missions revealed completely opposite situation, when synthesis prevails over decay (Kuzicheva and Simakov 1999). Diverse database from the last decade will be summarized in respect with chemical evolution processes and future space missions planning. Information gained from the spacecrafts during the scientifically planned experiments concerns not only biochemical data. The study of planets, their satellites and other celestial bodies widens our knowledge about the small solar system bodies and their possible role in the exchange of biological material. Having molecules within the mineral matrix, small cosmic bodies are transported to various regions, where ultraviolet irradiation may become important and alter the grain composition. UVC radiation may contribute to the formation of additional derivatives. This presumption coincides with our previous investigations concerning UV impact on prebiotic formation of the main biological molecules (Kuzicheva and Gontareva, 2003). Diverse irradiation types in different stages of space flight with possible occasion heating in close proximity to the Sun, could compensate the effects of low reagents concentration and temperature.

The importance of lab investigations should not be underestimated. They provide unique opportunity to study extraterrestrial organic chemistry by means of simulation experiments. Irradiation of solid samples, free or admixed with certain minerals, was tested in simulation experiments within the framework of the next space mission planning. The task of last investigations has been to work out strategy for the full-scale orbital experiment in order to avoid any mistakes and data loss. "Bion-M" flight is scheduled for the year 2010 and covers different aspects of prebiotic formation of organic molecules on mineral matrix triggered by space radiation in water-free conditions. In the framework of key FSP-2015 projects on fundamental space research (FSR) task is set to create new generation space crafts for sample-return missions ensuring the delivery of extraterrestrial matter to the Earth and bringing equipment into space for rigorous study of Solar system bodies. 
Kuzicheva, E. and Simakov, M. (1999). Abiogenic synthesis of nucleotides in conditions of space flight of biosputnik "Bion-11". Adv. Space Res., 23:387-391.

Kuzicheva, E. and Gontareva, N. (2001). Study of the prebiotic synthesis in context of exobiologycal investigations on Earth orbit. Adv. Space Res., 24:393-396.

Kuzicheva, E. and Gontareva, N. (2003). Astrobiological investigations on Russian space crafts. Astrobiology., V. 3: 253-262.

E-mail: ngontar@mail.cytspb.rssi.ru

\section{A Pre-biotic Nature of Complimentarity}

Andrey A. Ivanov

Vernadsky Institute of Geochemistry and Analytical Chemistry

Whatever the origin of life scenario was, there was no way for the Earth natural history to start without its specific pre-biotic, pre-biological, step. Which may and might this step be at the very Moment of the Beginning? And, after all, which was a key biological principle that had dramatically changed a lifeless image of the prehistoric Earth predestinating the most mart of its current natural history ? Without answering to this question, it is hardly possible to get the answer to the next one.

The critically essential mark of the matter's organization biological level is a conformational principle of interactions between biological systems as long as all biochemical processes, no exception found, are enzymatic and hence conformationdependent ones. Same way, the genome expression depends on the DNA conformational status. DNA consists of two polynucleotide chains, complimentary bound to each other by hydrogen bonds throughout all the length, which makes a known system of double helix (Ivanov and Galimov, 2007 and Ivanov, 2007). Genetic information is to be transferred due to replication of complimentary bases formed chains and, as far as it is known, this major principle is taken as universal for all pre-existing and currently functioning life forms. Therefore, an autonomy of the genetic information transfer might be guaranteed only in case of the complementary nucleotide pair existence which is, in turn, is one of the basic conditions needed for answering to the first question.

What is a nature of origin of the first informational molecules complimentary structures ? Certainly, once overlooking both random and regularity-formed routes of the possible processes of these macromolecules origin in a row, then just by definition, a preference should be given to the latter one. That choice might be made, particularly, due to the regularity format of the event expected.

A high level of the molecular structures conformational fitting could be provided by regression of polyheterocyclic compounds. To visualize that, let's imagine the woody pencil rupture sharp margins having an easily reached high-rank conformational inter-coupling fitting. Most probably, this clear and simple way was in fact chosen by nature known due its smart solutions for complex problems. Getting through analysis of an alternative way, i.e. the way of the complementary pair members separate origin along with a point of unbelievable degree of the conformational match randomness, then this way is definitely far more timeconsuming and far less trust-deserving. Even in a close look, this gives a reason to exclude the variant of single and consequent synthesis of abiogenic nucleotides. At 
least, the wide range chromato-mass-spectrometric studies on compounds obtained in a course of the pre-biotic abiogenic synthesis simulation allowed to find no one case of the complimentary nucleotide pair formation. On other hand, it has been proven that the heterocyclic compounds abiogenc synthesis is a rather reachable task (Lupatov, et al. 2006). From this standpoint, the single nucleotide synthesis focused simulation of abiogenic processes look not reasonable. However, the data obtained shows a direction for further studies devoted to the very first steps of the matter's biological evolution. One of these steps is a regression of polyheterocyclic compounds leading to formation of the mutually complimentary molecular structures.

Ivanov, A. and Galimov, E. (2007). Molecular isotopy of conformational interactions. Symposium on isotopic geochemistry named by A. Vinogradov, pages 44-45. Moscow, Russia.

Ivanov, A. (2007). Does the conformation of DNA depend on the difference in the isotope composition of it's threads. Russian Journal of Physical Chemistry, 6(1).

Lupatov, V. Strizhov, V. et al. (2006). Modeling of fusion reactions of the organic compounds in conditions of a primary atmosphere of the Earth. International symposium on molecular photonics. St. Petersburg, Russia.

E-mail: aiva@geokhi.ru

\title{
Racemization in Photodimerization of Solid Alanine Induced by Vacuum Ultraviolet Irradiation: Chiral Problem in Chemical Evolution
}

\author{
Yudai Izumi, Akiko Imazu, Aki Mimoto, Kazumichi Nakagawa
}

Graduate School of Human Development and Environment, Kobe University, Japan

L-rich amino acid was detected from Murchison meteorite (e.g. Cronin and Pizzarello, 1997). In chemical evolution from monomer to peptides induced by vacuum ultraviolet (VUV) light and/or X-ray, photoracemization of l-type amino acids is a serious problem. In this work, we examined photodimerization and photoracemization of solid l-alanine (Ala) in an attempt to examine whether the chirality of 1-Ala was preserved in chemical evolution.

We irradiated VUV light (wavelength $=172 \mathrm{~nm}$ ) onto l-Ala thin films at about $290 \mathrm{~K}$ in vacuum. After irradiation, all samples were dissolved with distilled water and analyzed by a high performance liquid chromatography (HPLC).

Fig. 1 shows chromatograms of irradiated 1-Ala film (curve (a)) and aqueous solution of marker molecules (curve (b)). The peak of d-Ala (around $17 \mathrm{~min}$ ), d-alanyl-l-alanine (D-L, around $25 \mathrm{~min}$ ), 1-alanyl-l-alanine (L-L, around $38 \mathrm{~min}$ ) and l-alanyl-d-alanine (L-D, around $41 \mathrm{~min}$ ) were found in curve (a). Thus we can write the equation as "l-Ala $+h \nu \rightarrow \mathrm{L}-\mathrm{L}+\mathrm{L}-\mathrm{D}+$ D-L + d-Ala." Amount of Gly and d-alanyl-d-alanine (D-D) was smaller than detection limit.

Production of L-D, D-L and d-Ala suggests that the chirality of 1-Ala was not preserved. In contrast, d-type amino acids were not found in the case of photolysis of 1-Asp (wavelength= $146 \mathrm{~nm}$ ) (Izumi et al., in print). Racemization is a critical problem in production of biomacromolecules (protein, DNA, RNA etc.). Therefore it is necessary to carry out the 
similar experiments using other amino acids and/or other energy sources in order to examine the "chiral stability" of amino acids and so on.

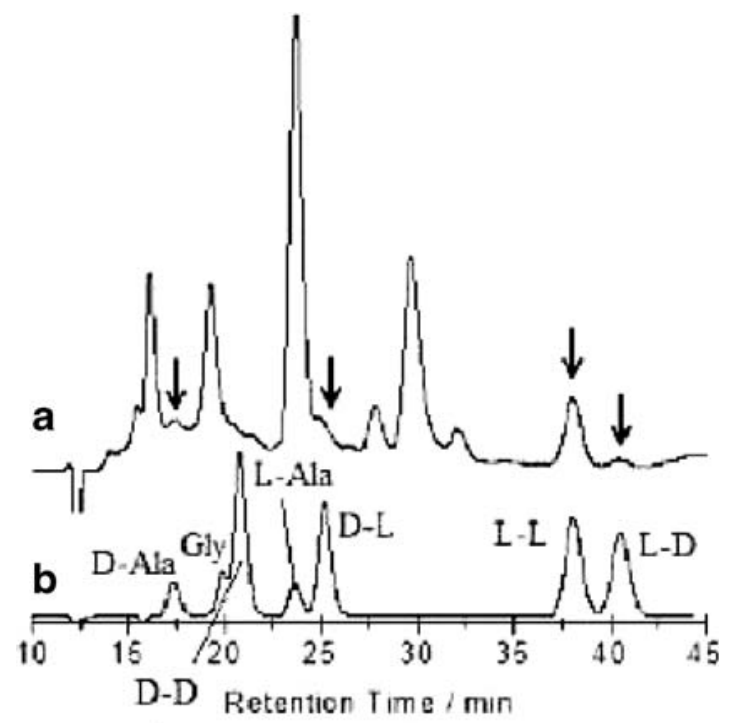

Fig.1 HPLC chromatograms.

Cronin, J. R. and Pizzarello, S. (1997). Enantiomeric excesses in meteoritic amino acids. Science 275: 951-955

Izumi, Y., Matsui, T., Koketsu, T. and Nakagawa, K. (in print). Preservation of homochirality of aspartic acid films irradiated with $8.5 \mathrm{eV}$ vacuum ultraviolet light. Radiation Physics and Chemistry.

E-mail: izumi@radix.h.kobe-u.ac.jp

The Diversity of the Original Prebiotic Soup: Re-analyzing the Original Miller-Urey Spark Discharge Experiments

\section{A. Johnson ${ }^{1}$, H.J. Cleaves ${ }^{2}$, J.L. Bada ${ }^{3}$, A. Lazcano ${ }^{4}$}

${ }^{1}$ Interdisciplinary Biochemistry Program, Indiana University, Bloomington, IN 47405; ${ }^{2}$ Geophysical Laboratory, Carnegie Institution of Washington, Washington D.C. 20015; ${ }^{3}$ Scripps Institution of Oceanography, University of California, San Diego, La Jolla, CA 92093-0212; ${ }^{4}$ Facultad de Ciencias, UNAM 04510 Mexico D.F., Mexico

On May 15th, 1953, a short paper by a graduate student named Stanley Miller appeared in the journal Science. It described the spark discharge formation of glycine, alanine and several other amino acids (Miller, 1953) from inorganic constituents thought to comprise the hypothesized reducing atmosphere of early Earth. Miller's work quite literally "sparked" the legitimization of the field of prebiotic chemistry; the basic molecules of life could, with relative ease, be synthesized from inorganic compounds thought to be abundant in the Earth's atmosphere 4.5 billion years ago. Darwin's "warm little pond" was no longer a hypothetical concept as much as a feasible scenario. 
Recently discovered samples from the original spark discharge experiments have been re-analyzed using HPLC-FD and LC-FD/ToF-MS in order to identify lesser constituents that would have been undetectable by analytical techniques 50 years ago. Using his original laboratory notebooks (Mandeville Special Collections, UCSD), we have reconstructed and identified the original fractions from his three thesis experiments The overall goal of this research was to identify lesser constituents of the original extracts that would have been undetectable by the ninhydrin-spray technique of the 1950s.

Results show the presence of several isomeric forms of aminobutyric acid, as well as serine, homoserine, isoserine, isovaline, valine, phenylalanine, ornithine, amino adipic acid, ethanolamine and other methylated and hydroxylated amino acids. These analyses identified the previously unknown compounds E, F and B1 (Miller, 1954; Miller, 1955) as a yet undetermined $\mathrm{C}_{4}$ amino acid, ethanolamine and $\beta$-amnoisobutyric acid, respectively. Both the diversity and yield increased in experiments utilizing a wateraspirating device designed to increase water vapor-gas flow rates delivered to the spark. Application of this experiment to early Earth would best mimic the intense lightning discharges that accompany volcanic eruptions. In this scenario, reduced and neutral gas species would be subjected to lightning, and thus exposed to localized discharge events prior to being rained out into tidal areas where products could undergo concentration events. The distribution of compounds formed in these experiments is significantly greater than previously published (Miller, 1954; Miller, 1955) and mimic the assortment of compounds detected in both Murchison (Botta and Bada, 2002) and CM meteorites (Glavin, et al. 2006). The addition of these several new amino acid and amine species to the previously reported spark discharge products will serve as a fitting final tribute to the founding father of prebiotic chemistry.

Botta, O. and Bada, J. L., (2002). Extraterrestrial organic compounds in meteorites. Surveys in Geophysics. 23: 411-467.

Glavin, D. P., Dworkin, J. P., Aubrey, A., Botta, O., Doty III, J. H., Martins, Z., and Bada, J. L. (2006). Amino acid analyses of Antarctic CM2 meteorites using liquid chromatography-time of flight-mass spectrometry. Meteorit. Planet. Sci. 41:889-902

Miller, SL. (1953). A production of amino acids under possible primitive earth conditions. Science, 117: 528-529

Miller, SL. (1954). A production of organic compounds under possible primitive earth conditions. PhD thesis. Department of Chemistry, University of Chicago.

Miller, SL. (1955). Production of some organic compounds under possible primitive earth conditions. Journal of American Chemical Society, 77: 2351-2361.

Miller, SL. Notebooks. Special Thanks to the Mandeville Special Collections Library, University of California, San Diego for their help in obtaining these original notebooks

E-mail: adpjohns@indiana.edu

\section{Amino Acids Interaction with Hydroxyapatite and UV-Vis Light: Primitive Earth Modeling}

\section{Seisuke Kano}

Natl Inst Adv Ind Sci \& Technol (AIST), Namiki1-2-1, Tsukuba, Ibaraki, Japan

Low molecular weight organic compounds, such as amino acids, which were generated by inorganic processes (Schlesinger and Miller, 1983) and/or around the primitive earth 
conditions (Kobayashi and Ponnamperuma, 1985), might be existed on the primitive earth effecting from the high temperature, high energy UV light, or radio wave irradiation. These low molecular weight compounds might be became large molecular compounds during such primitive earth environment. These compounds including amino acids might be increased their molecular weights and variations through several chemical processing, which were proposed a lots of researchers (Miyakawa, 2004) but few reports the effects of the UV-Vis light irradiation to the amino acids. In this study the affects were investigated of the UV-Vis lamp light irradiation to the amino acids solution with or without hydroxyapatite, HAp, which is one of the hydrothermal deposit mineral.

The test solutions were prepared by the amino acids standard solution (H-type, WAKO chem; $2.5 \mu \mathrm{mol} / \mathrm{ml}$ ) with citric acid sodium buffer solution ( $\mathrm{pH} 2.2$, WAKO chem.) measured up to $100 \mathrm{ml}$. Part of the test solution was added the HAp powder (672 $\mathrm{mg}$ ) and the other solution added the HAp powder without amino acids standard solution. These solutions put into Pyrex beakers and stirred during UV-Vis lamp light irradiation. The lamp located at $600 \mathrm{~mm}$ from the beakers and adjusted $400 \mathrm{~W}$ in the total power. The test solutions were inspected at just before light irradiation, second, fourth, seventh, ninth, and 11th days. The sampling solutions were analyzed by the amino acids analyzer (Shimadz Co. Ltd.). The precipitated samples including powders were separated to an upper solutions and powder compounds which were dried by vacuum dryer at room temperature and resolved with a hydrochloric acid solution. The resolved powder samples were filtering again and analyzed. The upper solution of the amino acids standard with HAp powder showed their amino acids concentrations were increased, excepting CYS, from 0.025 to $0.035 \mu \mathrm{mol} / \mathrm{ml}$ on average. From the precipitation of the amino acids standard with HAp powder the several amino acids were also detected but their concentrations were quite low, such as $0.005 \mu \mathrm{mol} / \mathrm{ml}$ on average. On the case of without HAp powder amino acids solution, the included amino acids concentrations were increased, excepting CYS, MET, and TYR. On the other hand, the HAp powder only mixed in the citric acid buffer solution, there were few organic compounds detected. These results might be indicated that the amino acids compounds were generated by UVVis light energy and also HAp powder effects, but HAp powder itself had few ability to generate amino acids compounds.

Kobayashi, K., and Ponnamperuma, C. (1985). Trace elements in chemical evolution. Origins of Life, 16:57-67.

Miyakawa, S. (2004). Origins of life and temperatures of the early earth, Viva Originor, 32:68-80.

Schlesinger, G. and Miller S. L. (1983). Prebiotic synthesis in atmospheres containing $\mathrm{CH}_{4}$, $\mathrm{CO}$, and $\mathrm{CO}_{2}$. Journal of Molecular Evolution, 19:383-390.

E-mail: s.kano@aist.go.jp

\section{Prebiotic Molecules Derived from Tholins}

Bishun N. Khare ${ }^{1,2,3}$, Christopher P. McKay ${ }^{1}$, Dale P. Cruikshank ${ }^{1}$, Yasuhito Sekine ${ }^{4}$, Patrick Wilhite $^{5}$, Tomoko Ishihara ${ }^{1,2}$, Lauren Tracy ${ }^{2,5}$, Katherine Lanier ${ }^{2,5}$, Delphine Nna-Mvondo ${ }^{6}$

${ }^{1}$ Carl Sagan Center, SETI Institute; ${ }^{2}$ Space Science Division, NASA Ames Research Center; ${ }^{3}$ Physics Department, San Jose State University; ${ }^{4}$ Department of Complexity 
Science and Engineering, University of Tokyo, 5-1-5 Kashiwanoha, Kashiwa, Chiba 2778561, Japan; ${ }^{5}$ Santa Clara University, Santa Clara, California; ${ }^{6}$ Centro de Astrobiologia (CAB)/CSIC-INTA, Ctra. de Ajalvir, km 4, Torrejon de Ardoz, Madrid, Spain

For over three decades tholins have been synthesized previously in the Laboratory for Planetary Studies at Cornell University and in recent years at NASA Ames Research Center from mixtures of the cosmically abundant gases $\mathrm{CH}_{4}, \mathrm{C}_{2} \mathrm{H}_{6}, \mathrm{NH}_{3}, \mathrm{H}_{2} \mathrm{O}, \mathrm{HCHO}, \mathrm{N}_{2}$, and $\mathrm{H}_{2} \mathrm{~S}$. The tholin synthesized by UV light or spark discharge on sequential and nonsequential pyrolysis GC-MS revealed hundreds of compounds and on hydrolysis produced a large number of amino acids including racemic protein amino acids. Optical constants have been measured of many tholins such as tholins produced from a condensed ice mixture of water and ethane at $77 \mathrm{~K}$, poly $\mathrm{HCN}$, tholin synthesized by sparking an equimolar mixture of $\mathrm{CH}_{4}$ and $\mathrm{NH}_{3}$ with $2.5 \%$ water vapor crudely simulating the lower clouds of Jupiter, and Titan tholin produced on electrical discharge through a mixture of $90 \% \mathrm{~N}_{2}$ and $10 \% \mathrm{CH}_{4}$ simulating the upper atmosphere of Titan intercepted by magnetospheric charged particles of Saturn. Optical constants of Titan tholin for the first time are measured from soft x-rays to microwave frequencies (Khare et al., 1984) that on hydrolysis produced 16 protein amino acids, urea, and non-protein amino acids. The amino acids were racemic (Khare et al., 1986). The dark brown tholin in the lower clouds of Jupiter on treating with $\mathrm{NH}_{4} \mathrm{OH}$ of $\mathrm{pH}=13$ at room temperature over a period of $30 \mathrm{~h}$ revealed protein, non-protein, and polyamino acids. Several to over a dozen amino acids in the polyamino acid peaks were identified. Jupiter tholin as well as Titan tholin revealed the presence of polycyclic aromatic hydrocarbons (PAHs) that are considered to be the most abundant gaseous species in the interstellar medium (Sagan et al., 1993). PAHs in ices on photolysis produce biologically relevant molecules such as alcohols, quinones, and ethers (Bernstein et al., 1999). Here we report the absorption of gases on tholin produced in Titan's atmosphere in the temperature range 135 to $178 \mathrm{~K}$ by magnetospheric charged particles, and passing through lower temperature $(70 \mathrm{~K})$ and finally to the ground at $95 \mathrm{~K}$. While descending to the ground, tholin particles get coated with other species (ions, radicals etc) and processed along the way by other sources of energy such as long UV and cosmic rays. It is therefore expected that the stable products of $\mathrm{CH}_{4}$ photolysis react with Titan tholin to recycle the $\mathrm{CH}_{4}$ supply in Titan's atmosphere. Further more, the reactions of gaseous $\mathrm{C}_{2} \mathrm{H}_{6}$ with the reactive materials on the surface of the tholin could incorporate atmospheric $\mathrm{C}_{2} \mathrm{H}_{6}$ into the tholin and therefore might reduce the deposition rate of $\mathrm{C}_{2} \mathrm{H}_{6}$ onto the ground of Titan.

Bernstein, M.P., Sanford, S.A., Allamandola, L.J., Gillette, J.S., Clemett, S.J., Zare, R.N. (1999). UV irradiation of polycyclic aromatic hydrocarbons in ices: Production of alcohols, quinines, and ethers. Science 283, 1135-1138

Khare, B.N., Sagan, C., Arakawa, E.T., Suits, F., Callott, T.A., Williams, M.W. (1984). Optical constants of organic tholins produced in a simulated Titanian atmosphere: From soft $\mathrm{X}$-ray to microwave frequencies. Icarus, 60: 127-137.

Khare, B.N., Sagan, C., Ogino, H., Nagy, B., Er, C., Schram, K.H., Arakawa, E.T. (1986). Amino acids derived from Titan Tholins. Icarus, 68: 176-184

Sagan, C., Khare, B.N., Thompson, W.R., McDonald, G.D., Wing, M.R., Bada, J.L., VoDinh, T., Arakawa, E.T. (1993). Polycylic aromatic hydrocarbons in the atmosphere of Titan and Jupiter. ApJ, 414: 399-405.

E-mail: Bishun.N.Khare@nasa.gov 


\section{Interstellar Origins of Complex Amino Acid Precursors with Large Molecular Weights}

Kensei Kobayashi ${ }^{1}$, Toshinori Taniuchi ${ }^{1}$, Takeo Kaneko ${ }^{1}$, Satoshi Yoshida ${ }^{2}$, Yoshinori Takano $^{3}$, Jun-ichi Takahashi ${ }^{4}$

${ }^{1}$ Yokohama National University; ${ }^{2}$ National Institute for Radiological Studies; ${ }^{3}$ Japan Agency for Marine-Earth Science and Technology; ${ }^{4}$ NTT Microsystem Integration Laboratories

Complex organic compounds with large molecular weights have been detected in carbonaceous chondrites and comets. Recent works suggested that these complex organics were formed in low temperature environments (Nakamura-Messenger et al. We irradiated mixtures of simple molecules found in interstellar environments such as carbon monoxide, methanol, ammonia and water with high energy particles, and characterized the products.

A mixture of methanol, ammonia and water was irradiated with high-energy heavy ions (e.g., $290 \mathrm{MeV} / \mathrm{u} \mathrm{C}^{6+}$ ) from HIMAC accelerator (NIRS, Japan) at $77 \mathrm{~K}$ or ambient temperature. A mixture of carbon monoxide, ammonia and water was irradiated with $3 \mathrm{MeV}$ protons from a van de Graaff accelerator at 10-20 K (Kasamatsu et al, 1997) or ambient temperature. The products were acid-hydrolyzed, and amino acids were analyzed by HPLC and/or GC/MS. Unhydrolyzed products were analyzed by GFC, pyrolysis-GC/ MS, TEM, etc.

Racemic mixtures of amino acids were detected in all the irradiation products. There were little difference in energy yields of amino acids (after hydrolysis) between ambient irradiation and low-temperature irradiation. Molecular weights of unhydrolyzed products are a few thousands, and gave a wide variety of molecules including heterocyclic compounds by pyrolysis-GC/MS. It was suggested that complex amino acid precursors with large molecular weights could be formed in ice mantles of interstellar dusts in dense clouds by action of cosmic rays.

The complex amino acid precursors were much more stable than free amino acids against radiation, heating and high-velocity impacts. They showed amorphous particulate cottony images of high-molecular-weight complex organics by TEM and AFM. When they were irradiated with circularly polarized UV light (CPL) from a synchrotron and then acid-hydrolyzed, enantiomeric excesses were observed, and amino acid yields before and after CPL was almost the same (Takano et al., 2007). These results implied that the not only amino acids but also seeds of their homochirality were formed in interstellar cold environments, and they were delivered by extraterrestrial bodies to Earth.

Kasamatsu, T., Kaneko, T., Saito and Kobayashi, K. (1997). Formation of organic compounds in interstellar media with high energy particles. Bull. Chem. Soc. Jpn., 70: 1021-1026.

Nakamura-Messenger, K., Messenger, S., Keller, L. P., Clemett, S. J. and Zolensky, M. E. (2006). Organic globules in the Tagish Lake Meteorite: Remnants of the protosolar disk. Science, 314:1439-1442.

Takano, Y., Takahashi, J., Kaneko, T., Marumo, K. and Kobayashi, K. (2007). Asymmetric synthesis of amino acid precursors in interstellar complex organics by circularly polarized light. Earth Planet. Sci. Lett., 254: 106-114.

E-mail: kkensei@ynu.ac.jp 


\section{Investigation of Laser Plasma Chemistry in $\mathrm{CO}_{2}-\mathrm{N}_{2}-\mathrm{H}_{2} \mathrm{O}$ Using ${ }^{18} \mathrm{O}$ Labeled Water}

Martin Ferus ${ }^{1,2}$, Petr Kubelík ${ }^{1,2}$, Libor Juha ${ }^{2}$, Svatopluk Civišs ${ }^{1}$

${ }^{1}$ J. Heyrovsky Institute of Physical Chemistry, Academy of Sciences of the Czech Republic, v.v.i., Dolejškova 3, 18223 Prague 8, Czech Republic; ${ }^{2}$ Institute of Physics, Academy of Sciences of the Czech Republic, v.v.i., Na Slovance 2, 18223 Prague 8, Czech Republic

This work is focused on chemical reactions in organic gas mixtures in high-power laser induced plasma which may lead to formation of small organic compounds. Large-scale plasma was created in gas mixtures containing carbon monoxide by high-power laser-induced dielectric breakdown (LIDB). The composition $\left(\mathrm{CO}-\mathrm{N}_{2}-\mathrm{H}_{2} \mathrm{O}\right)$ of used mixtures corresponded to a cometary and/or meteoritic impact into the Earth's early atmosphere (Babánková D. et al. 2006). A multiple-centimeter-sized fireball was created by focusing a single $85 \mathrm{~J}, 450$ ps nearinfrared laser pulse into the centre of a 15-L gas cell. The LIDB plasma chemical evolution was investigated by optical emission spectroscopy (OES) with temporal resolution (Babánková D. et al. 2006). The chemical consequences of laser-produced plasma generation in a $\mathrm{CO}-\mathrm{N}_{2}-\mathrm{H}_{2} \mathrm{O}$ mixture were investigated using high resolution Fourier transform infrared absorption spectroscopy (FTIR) and gas chromatography (GC) (Civiš S. et al. 2008). The reaction mechanism of $\mathrm{CO}_{2}$ formation was investigated using water isotopomer $\mathrm{H}_{2}{ }^{18} \mathrm{O}$.

\section{Acknowledgements}

This work was financially supported by Grant Agency of the Czech Republic (grant No. 203/06/1278) and the Czech Ministry of Education (grants LC510, LC528 and LA08024).

Babánková D., Civiš S., Juha L., Bittner M., Cihelka J., Pfeifer M., Skála J., Bartnik A., Fiedorowicz H, Mikolajczyk J., Šedivcová T. (2006). Optical and x-ray emission spectroscopy of high-power laser-induced dielectric breakdown in molecular gases and their mixtures. Journal of Physical Chemistry A, 110:12113-12120.

Babánková D., Civiš S., Juha L. (2006). Chemical consequencies of laser-induced breakdown in molecular gases. Progress in Quantum Electronics, 30:75-88.

Civiš S., Babánková D., Cihelka J., Sazama P., Juha L. Spectroscopic investigation of highpower laser-induced dielectric breakdown in gas mixtures containing carbon monooxide. To appear in the Journal of Physical Chemistry A.

E-mail: petr.kubelik@centrum.cz

\section{Dipeptide Formation from Leucine, Methionine and Arginine Under Primordial Earth Conditions}

Feng $\mathrm{Li}^{1,2}$, Daniel Fitz ${ }^{1}$, Bernd M. Rode ${ }^{1}$

${ }^{1}$ Faculty of Chemistry and Pharmacy, Institute of General, Inorganic and Theoretical Chemistry, University of Innsbruck, Innrain 52a, A-6020 Innsbruck, Austria; ${ }^{2}$ Department of Earth Sciences, University of Oxford, Parks Road, Oxford OX1 3PR, United Kingdom

The Salt-Induced Peptide Formation (SIPF) reaction, discovered in the late 1980s (Schwendinger and Rode, 1989) and implemented through drying-and-wetting cycles with the help of divalent copper ions and sodium chloride in aqueous solution, has repeatedly 
shown to be a universal and feasible pathway for simple peptide formation under primordial earth conditions (Rode, 1999) and also casts light on the puzzle of the origin of biohomochirality especially in case of amino acids with aliphatic side chains (Fitz, et al. 2007).

In the present work, three functionally interesting amino acids, namely, hydrophobic leucine, sulphur-containing methionine ( $\mathrm{Li}$, et al. 2008) and guanidine-capped arginine, were investigated with regard to their dipeptide yields and the catalytic effects of glycine, L- and D-histidines respectively. Results indicate that methionine has the best reactivity for dimerisation in the SIPF reaction, while for leucine, a remarkable preference for the Lenantiomer could be detected just as for its other aliphatic congeners, despite of de facto poor yields. Concerning the catalytic amino acids, dileucine yields show a positive feedback on all three catalysts, whereas both histidine enantiomers are much more effective for diarginine formation than glycine, and none of them contributes to boost the methionine reaction except at low concentrations.

The differences above can be attributed to several interacting factors such as the complex-formation coefficient (Shoukry, et al. 1997), the polarity, the size, the hydrophobicity, and the nucleophilicity and electrophilicity etc.

Fitz, D., Reiner, H., Plankensteiner, K., and Rode, B. M. (2007). Possible origins of biohomochirality. Current Chemical Biology, 1(1): 41-52.

Li, F., Fitz, D., Fraser, D. G., and Rode, B. M. (2008). Methionine peptide formation under primordial earth conditions. Journal of Inorganic Biochemistry, 102(5-6): 1212-1217.

Rode, B. M. (1999). Peptides and the origin of life. Peptides, 20(6): 773-786.

Schwendinger, M. G. and Rode, B. M. (1989). Possible role of copper and sodium chloride in prebiotic formation of peptides. Analytical Sciences, 5(4): 411-414.

Shoukry, M. M., Khairy, E. M., and Khalil, R. G. (1997). Binary and ternary complexes involving copper(II), glycyl-DL-leucine and amino acids or amino acids esters: hydrolysis and equilibrium studies. Transition Metal Chemistry, 22(5): 465-470.

E-mail: feng.li@worc.ox.ac.uk

\section{Polymerisation of Amino Acids on Oxide Surfaces}

\section{Lopes}

Laboratoire de Réactivité de Surface-UMR-7609, Université Pierre et Marie Curie, Paris, France.

Amino acids are essential components of living matter and the description of their initial polymerisation to form peptides remains a major problem in the establishment of reasonable origins of life scenarii (Lambert, 2008). It has been proposed (Bernal, 1950) that the prebiotic polymerisation of amino acids occurred in the adsorbed state on mineral oxide surfaces because this reaction is thermodynamically unfavourable in aqueous phase. This could have occurred at the surface of the primitive earth and/or on interstellar material.

Here we present a comparative study of adsorption and thermal activation of different amino acids on different common oxides such as silica and titanium oxide. Several amino acids carrying different side chains, and therefore having a different acid-base speciation, were considered. The adsorption isotherms were established by HPLC, and the adsorbed molecules were characterized by IR spectrometry (Meng et al., 2004) and ${ }^{13} \mathrm{C}$ and ${ }^{15} \mathrm{~N}$ 
solid-state NMR (Stievano et al., 2007). These techniques were also employed, together with thermogravimetry and mass spectrometry to follow the thermal activation of the adsorbed amino acids in the adsorbed state. Experimental data were systematically compared with the results of molecular modelling by DFT and molecular dynamics (Lomenech et al., 2005; Costa et al., 2007 and 2008).

On the basis of the various spectroscopic signatures of the complex formed between the amino acids and their adsorbing oxide surface, we propose different mechanisms and sites of adsorption. In particular, the study of the glycine/silica system in anhydrous conditions and in aqueous solutions at different $\mathrm{pHs}$ clearly indicates that water simultaneously influences the speciation of adsorbed glycine and the mechanism of adsorption. Depending on the conditions of adsorption, glycine can be present in four different forms: bulk $\alpha$ and $\beta$-glycine and glycine zwitterions molecularly adsorbed as specifically hydrogen-bonded adducts on clusters of silanol groups in aqueous conditions, and molecularly adsorbed neutral glycine at low water activity. These forms have different thermal reactivities regarding the condensation of the peptide bond, which can be followed in situ with solidstate NMR. On silica, the adsorbed molecules form peptide bonds at temperatures considerably lower than for the crystalline amino acid, producing mainly cyclic dimers (diketopiperazines), which strongly interact with the surface of silica but can be opened to linear peptides when high water activities are restored. On titania, amino acids are adsorbed as coordinative complexes which are too stabilised to show a tendency toward thermal polymerisation.

The thermal activation of different amino acids (glycine, glutamic acid and leucine) leading to the formation of peptide bonds was studied on silica and on titania surfaces. Selectivities in adsorption were demonstrated in the (lysine + glycine) system (Stievano et al., 2007), and in the (leucine + glutamic acid) system (Lambert et al., 2008); they depend on the nature of the surface, the $\mathrm{pH}$ of the solution and the amount of adsorbed amino acid. Peptide formation selectivities seem to be present as well in the second system.

We discuss the relevance of these results for the formation of peptides in prebiotic scenarios.

Bernal, D. (1951). The Physical basis of life. Proc. Phys. Soc., 61(10):597-618.

Costa, D., Lomenech, C., Meng, M., Stievano, L., and Lambert, J. F. (2007).Microsolvation of Glycine by Silanol Ligands and Water: A DFT Study. Theochem, 806 (1-3), 253-259.

Costa, D., Tougerti, A., Tielens, F., Gervais, C., Stievano, L., and Lambert, J. F. (2008). Exploring the Adsorption of Microsolvated Glycine on the Surface of Amorphous Silica with Periodic DFT. Submitted.

Lambert, J. F. (2008). Adsorption and polymerization of amino acids on mineral surfaces. Orig. Life Evol. Biosph., in the press

Lambert, J. F., Stievano, L., Lopes, I., Gharsallah, M., and Piao, L. Y. (2008). The fate of amino acids adsorbed on mineral matter, submitted to Planet. Space Sci.

Lomenech, C., Bery, G., Costa, D., Stievano, L., and Lambert, J. F. (2005). Theoretical and experimental study of the adsorption of neutral glycine on silica from the gas phase. ChemPhysChem, 6, 1061-1070.

Meng, M., Stievano, L., and Lambert, J. F. (2004). Adsorption and thermal condensation mechanisms of amino acids on oxide supports. 1.Glycine on Silica. Langmuir, 20:914-923. Stievano, L., Piao, L. Y., Lopes, I., Meng, M., Costa, D., and Lambert J. F. (2007). Glycine and lysine adsorption and reactivity on the surface of amorphous silica. European Journal of Mineralogy, 19:321-331

E-mail: irene.lopes@upmc.fr 


\title{
Interaction of Amino Acids in Mineral Surfaces and Their Relevance in Chemical Evolution
}

\author{
L. López-Esquivel Kranksith ${ }^{1}$, A. Negrón-Mendoza ${ }^{1}$, G. Cocho-Gil ${ }^{2}$, S. Ramos-Bernal ${ }^{1}$ \\ ${ }^{1}$ Instituto de Ciencias Nucleares; ${ }^{2}$ Instituto de Física Universidad Nacional Autónoma de \\ Mexico (UNAM) Mexico D.F.
}

Laboratory studies have been carried out simulating the chemical evolution stage of the possible conditions on the primitive Earth. Experiments with various solids (silica, clays, and aluminum-silicates) have shown that they could act not only as surfaces of support, but also as catalysts (Ferris and Ertem, 1992).

On the other hand, studies of interstellar matter reveal the presence of complex organic molecules such as polycyclic aromatic hydrocarbons (PAH), fullerenes and carbon nanotubes (CNTs) (Georgakilas, et al. 2000), acetamide (a precursor of amino acids), simple amino acids and sugars. The questions then arise: How these molecules can survival? Which are the mechanisms involved? In an attempt to answer these questions a series of experiments were undertaking with selected compounds and we study the survival of molecules, such as amino acids, in a hostile high radiation field while they are adsorbed environment (Kawasaki, et al., 2006).

To this end, we analyzed the adsorption of amino acids in clay mineral, charcoal (PAC) and carbon nanotubes (CNTs) as possible phases that may ocurred in the primitive Earth or in extraterrestrial environments. We also studied further the behavior of amino acids adsorbed in these solid surfaces, in different conditions of $\mathrm{pH}$, concentration and levels of irradiation, simulating a high radiation field in the early Earth conditions.

The analisis of the samples were performed by UV-vis spectroscopy, X-rays and infrared spectroscopy.

Trials adsorption with, Aspartic (Asp) and Glutamic (Glu) acids in sodium montmorillonite were conducted for different times of contac. The adsorption for Asp was of $98 \%$ and for Glu was of $60 \%$. In the case of Glu, an interest phenomenom took place and interaction with clay generates a visible coloration lemon-yellow in the clay. This may be related to the interactions between cationic links with clay and the molecular structure a this amino acid. It is also important to emphasize that this clay could promote the catalysis of other compounds, using as a precursor Glu. The complex clay-Glu, may form in this condition pyroglutamic acid (2-oxotetrahidropirrol 5-carboxylic acid), a chemical form of internal protection of glutamic acid, which can be obtained relatively easily, from a catalytic dehydration reaction (Yun, et al., 1998).

Trials adsorption in PAC with Asp and Glu have shown that these compounds rapidly adsorbed above the $60 \%$. This is because the properties of this surface-volume solid. Finally, the adsorption in other surfaces like in CNT was tested. For the study single wall (SWNT), double wall (DWNT) and multiple walls (MWNT) were tested with Asp, having a relatively rapid at different $\mathrm{pH} \AA \frac{1}{2} \mathrm{~s}$.

To study the possible survival of molecules in a high radiation field, in particular amino acids adsorbed in a solid surface, the irradiation of sistem solid surface-amino acid was undertaken. Preliminary results $\gamma$-irradiation of system Asp-clay will be discussed.

The relevance of this work is to explain the possible contribution of solids (clay, PAC and CNTs) as shields for the adsorbed organic compounds against external sources of energy. 
Ferris, J. P. and Ertem, G. (1992). Oligomerization Reactions of Ribonucleotides on Montmorillonite: Reaction of the 5' Phosphorimidazolide of Adenosine. Science, 257: 1387-1389.

Georgakilas, V., Tagmatarchis, N. D., Pantarotto, A., Bianco, J. P., Briand, M., and Prado, M. (2002). Amino Acid Functionalisation of Water Soluble Carbon Nanotubes. Chemical Communications, 3050-3051.

Kawasaki, T., Hatase, K., Fujii, Y., Jo, K., Soai, K., and Pizzarello, S. (2006). The Distribution of Chiral Asymmetry in Meteorites: An Investigation using Asymetric Autocatalytic Chiral Sensors. Geochimica et Cosmochimica Acta, 70: 5395-5402.

Yun, B. S., Ryo, I. J., Lee, I. K., and Yoo, I. D. (1998). Tetahedron, 54: 1515.

E-mail: laura,kranksith@nucleares.unam.mx

\section{SIFT-MS Analysis of Molecular Gas Mixtures Exposed to High-Power Laser Plasmas: Laboratory Simulation of High-Energy-Density Events in Early Earth's Atmospheres}

Kristana Sovová ${ }^{1}$, Irena Matulková ${ }^{1}$, Michal Kamas ${ }^{1}$, Kseniya Dryahina ${ }^{1}$, Patrik Španěl ${ }^{1}$, Libor Juha $^{2}$, Svatopluk Civiš ${ }^{1}$

${ }^{1}$ J. Heyrovsky Institute of Physical Chemistry, Academy of Sciences of the Czech Republic, v.v.i., Dolejškova 3, 18223 Prague 8, Czech Republic; ${ }^{2}$ Institute of Physics, Academy of Sciences of the Czech Republic, v.v.i., Na Slovance 2, 18221 Prague 8, Czech Republic

The main goal of this study was to synthesize simple organic molecules which can simulate the prebiotic synthesis of bioorganic compounds (Takahashi, et al. 2005; Civiš, et al. 2004). Large-scale plasma (Jungwirth, et al. 2001) (pulse energy about $100 \mathrm{~J}$, wavelength 1,315 nm, pulse duration $0.5 \mathrm{~ns}$ ) was formed by high-power laser-induced dielectric breakdown (LIDB) in molecular $\mathrm{CH}_{4}-\mathrm{N}_{2}-\mathrm{D}_{2} \mathrm{O}\left(1: 1: 10 \mathrm{ml}\right.$ - similar to atmosphere of Titan) and $\mathrm{CO}-\mathrm{N}_{2}-\mathrm{D}_{2} \mathrm{O}$ and $\mathrm{CO}-\mathrm{N}_{2}-\mathrm{H}_{2} \mathrm{O}(1: 1: 1 \mathrm{ml}$ - simulation of the prebiotic terrestrial atmosphere) gaseous mixtures for simulation of chemical consequences of high-energy density events such as lightning or impacts of extra-terrestrial bodies in the Earth's atmospheres.

The chemical consequences of the laser-produced plasma generation gaseous mixtures was analysed using Selected ion flow tube mass spectrometry (SIFT-MS) (Smith and Španěl, 2005) as a complementary method to the optical emission spectroscopy, gas chromatography and high resolution FTIR spectroscopy. Multiple mass spectra have been acquired for each sample alternating the three precursors $\left(\mathrm{H}_{3} \mathrm{O}^{+}, \mathrm{NO}^{+}\right.$and $\left.\mathrm{O}_{2}{ }^{+}\right)$. The difference between the mass spectra before and after irradiation is dramatic clearly testifying that multiple new compounds have been generated by the chemistry induced by the radiation.

The SIFT-MS analysis proved formation of hydrogen cyanide, acetylene, acetone, methanol, ethanol, methane, ethane, propene, propane, butane, butadiene, pentadiene, cyanoacethylene and pentacyanopolyene in the $\mathrm{CH}_{4}-\mathrm{N}_{2}-\mathrm{D}_{2} \mathrm{O}$ mixture (Kamas, 2007). The $\mathrm{CO}-\mathrm{N}_{2}-\mathrm{D}_{2} \mathrm{O}$ and $\mathrm{CO}-\mathrm{N}_{2}-\mathrm{H}_{2} \mathrm{O}$ mixtures provide under the same experimental conditions significantly lower concentrations of formed molecules including (hydrogen cyanide, nitrogen oxides, fulminic acid, etc.).

\section{Acknowledgements}

This work was financially supported by Grant Agency of the Czech Republic (grant No. 203/06/1278) and the Czech Ministry of Education (grants LC510, LC528 and LA08024). 
Civiš S., Juha L., Babánková D., Cvačka J., Frank O., Jehlička J., Králíková B., Krása J. Kubát P., Muck A., Pfeifer M., Skála J. and Ullschmied J. (2004). Amino acid formation induced by high-power laser in $\mathrm{CO}_{2} / \mathrm{CO}-\mathrm{N}_{2}-\mathrm{H}_{2} \mathrm{O}$ gas mixtures. Chem. Phys. Lett., 386:169-173.

Jungwirth K., Cejnarova A., Juha L., Kralikova B., Krasa J., Krousky E., Krupickova P., Laska L., Masek K., Mocek T., Pfeifer M., Prag A., Renner O., Rohlena K., Rus B., Skala J., Straka P., Ullschmied J. (2001). The Prague Asterix Laser System. Physics of Plasma, 8:2495-2501.

Kamas M. (2007). BSc thesis, Department of Physical and Macromolecular Chemistry, Charles University in Prague.

Smith D. and Španěl P. (2005). Selected ion flow tube mass spectrometry (SIFT-MS) for on-line trace gas analysis. Mass. Spectrom. Rev., 24:661-700.

Takahashi, J., Masuda, H., Kaneko, T., Kobayashi, K., Saito, T. and Hosokawa, T. (2005). Photochemical abiotic synthesis of amino-acid precursors from simulated planetary atmospheres by vacuum ultraviolet light. J. Appl. Phys., 98:024907-024913.

E-mail: irena.matulkova@jh-inst.cas.cz

\section{Efficient Synthesis of Pyrimidines and Triazines from Urea and Methane in Ice Matrix}

\section{Cesar Menor-Salván, Marta Ruiz-Bermejo, Susana Osuna-Esteban, Sabino Veintemillas-Verdaguer}

Centro de Astrobiología (CSIC-INTA). Torrejón de Ardoz (Madrid), 28850, Spain

The prebiotic synthesis of nucleic acid bases is a central issue in the proposal of self-assembly of nucleic acids and still is in debate. Cytosine and uracil are synthesized from cyanoacetylene, or its hydrolysis product cyanoacetaldehyde, and cyanate or urea (Ferris et al. 1968; Ferris et al. 1974, Robertson and Miller, 1995). On the other hand, the generation of cyanoacetylene by spark discharges in methane/nitrogen atmosphere has been demonstrated (Sanchez et al. 1966) and it is present in the atmosphere of Titan, comets and interstellar medium (Clarke and Ferris, 1997). Recently, the prebiotic relevance of pyrimidine synthesis from urea and cyanoacetylene was discussed (Shapiro, 1999, 2002). The main concerns are the reactivity and unstability of reactants, the problem of dilution and the possibility of cross reactions with amino acids (glycine is one of the main products obtained in experiments spark discharges). To overcome these problems, it has been hypothesized that water freezing could generate adequate conditions for the reaction, thanks to the exclusion of solutes to concentrated interstitial brines in the ice matrix (Orgel, 2004). Following this hypothesis, cytosine and uracil were synthesized from cyanoacetaldehyde and urea in freezing solution (Cleaves et al. 2006).

Here we report an efficient synthesis of cytosine and uracil from urea $0.1 \mathrm{M}$ in water and subjected to freeze-melt cycles during one week, under methane/nitrogen/hydrogen atmosphere, using spark discharges as energy source during the first $72 \mathrm{~h}$ of experiment. The analysis by GC/MS of the product shows, from major to minor concentrations, the synthesis of cyanuric acid, ammeline, the pyrimidines uracil, cytosine and 2,4diaminopyrimidine, ammelide, melamine and adenine. Amino acids, carboxylic acids and polycyclic aromatic hydrocarbons were also detected. Interestingly, we did not find insoluble organics. In conclusion, the prebiotic synthesis of pyrimidines is possible under methane atmospheres in freezing urea solutions. The high efficient synthesis of triazines plus the possible role of triazines as purine/pyrimidine mimics (Hysell et al. 2005) opens an interesting way for study.

Clarke, D.W. and Ferris, J. (1997). Titan haze: structure and properties of cyanoacetylene and cyanoacetylene-acetylene photopolymers. Icarus, 127:158-172. 
Cleaves, H.J., Nelson, K.E. and Miller S. (2006). The prebiotic synthesis of pyrimidines in frozen solution. Naturwissenschaften, 93(5):228-231.

Ferris, J., Sanchez, R. and Orgel, L. (1968). Studies in prebiotic synthesis. III. Synthesis of pyrimidines from cyanoacetylene and cyanate. J. Mol. Biol. 33:693-704.

Ferris, J., Zamek, O.S., Altbuch, A.M. and Freiman M. (1974). Chemical evolution. 18. Synthesis of pyrimidines from guanidine and cyanoacetaldehyde. J. Mol. Evol. 3:301-309. Hysell, M., Siegel, J.S., and Tor Y. (2005). Synthesis and stability of exocyclic triazine nucleosides. Org. Biomol.Chem., 3:2946-2952.

Orgel, L. (2004). Prebiotic adenine revisited: eutectics and photochemistry. Orig. Life Evol. Biosph., 34:361-369.

Robertson, M. and Miller, S. (1995). An efficient prebiotic synthesis of cytosine and uracil. Nature, 375:772-774.

Sanchez, R., Ferris, J. and Orgel, L. (1966). Conditions for purine synthesis: did prebiotic synthesis occur at low temperatures? Science 153:72-73.

Shapiro, R. (1999). Prebiotic cytosine synthesis. A critical analysis and implications for the origin of life. Proc. Natl. Acad. Sci. USA., 96:4396-4401.

Shapiro, R. (2002). Comments on 'concentration by evaporation and the prebiotic synthesis of cytosine'. Orig. Life Evol. Biosph. 32:275-278.

E-mail: menorsc@inta.es

\section{Photochemical Evolution of Simple Molecules on the Primitive Earth Under Simulated Prebiotic Conditions}

Daniele Merli ${ }^{1}$, Daniele Dondi ${ }^{1}$, Luca Pretali, ${ }^{2}$ Maurizio Fagnoni ${ }^{2}$, Angelo Albini ${ }^{2}$, Antonella Profumo ${ }^{1}$, Nick Serpone

${ }^{1}$ Dipartimento di Chimica Generale, Universita' di Pavia, via Taramelli 12, 27100 Pavia, Italy; ${ }^{2}$ Dipartimento di Chimica Organica, Universita' di Pavia, via Taramelli 10, 27100 Pavia, Italy; 'Professor Emeritus, Concordia University, Montreal, and Visiting Professor, Universita' di Pavia.

A series of prebiotic mixtures of simple molecules, sources of $\mathrm{C}, \mathrm{H}, \mathrm{N}$, and $\mathrm{O}$, were examined under conditions that may have prevailed during the Hadean (4.6-3.8 billion years), namely an oxygen-free atmosphere and a significant UV radiation flux over a large wavelength range due to the absence of an ozone layer (Lazcano and Miller, 1996; Chyba, 2005; Tian et al.; 2005). Mixtures contained a C source (methanol, acetone or other ketones), a $\mathrm{N}$ source (ammonia or methylamine), and an $\mathrm{O}$ source (water) at various molar ratios of C:H:N:O (Ehrenfreund and Charnely; 2007; Dondi et al., 2007). When subjected to UV light or heated for periods of 7 to 45 days under an argon atmosphere, they yielded a narrow product distribution of a few principal compounds. Different initial conditions produced different distributions. The nature of the products was ascertained by gas chromatographic-mass spectral analysis (GC-MS). UVC irradiation of an aqueous methanol-ammonia-water prebiotic mixture for 14 days under low UV dose $\left(6 \times 10^{-2}\right.$ Einstein) produced methylisourea, hexamethylenetetramine (HMT), methyl-HMT and hydroxy-HMT, whereas under high UV dose (45 days; $1.9 \times 10^{-1}$ Einstein) yielded only HMT (Hagen et al., 1979). By contrast, the prebiotic mixture composed of acetoneammonia-water produced five principal species with acetamide as the major component; thermally the same mixture produced a different product distribution of four principal species. UVC irradiation of the $\mathrm{CH}_{3} \mathrm{CN}-\mathrm{NH}_{3}-\mathrm{H}_{2} \mathrm{O}$ prebiotic mixture for 7 days gave 
mostly trimethyl-s-triazine, whereas in the presence of two metal oxides $\left(\mathrm{TiO}_{2}\right.$ or $\left.\mathrm{Fe}_{2} \mathrm{O}_{3}\right)$ also produced some HMT; the thermal process yielded only acetamide.

Chyba, C. F. (2005). Atmospheric Science:Rethinking Earth's Early Atmosphere. Science, 308:962-963

Ehrenfreund, P., and Charnley, S.B., (2000). Organic Molecules in the Interstellar Medium, Comets, and Meteorites: A voyage from dark clouds to the early Earth. Annu. Rev. Astron. Astrophys., 38:427-483

Hagen, W., Allamandola, L. J., and Greenberg, J. M. (1979). Interstellar molecule formation in grain mantles: the laboratory analog experiments, results and implications. Astrophys. Space Sci., 65:215-240

Lazcano, A. S., and Miller, S. I. (1996). The origin and early evolution review of life: Prebiotic chemistry, the pre-RNA world and time, Cell, 85:793-798

Tian, F., Toon, O. B., Pavlov, A. A., and Sterck, H. D. (2005). A hydrogen-rich early Earth atmosphere, Science, 308:1014-1017

E-mail: daniele.merli@libero.it

\title{
Surface-Enhanced Raman Investigation on the Peptide Formation by Adsorption of Glycine and Diglycine on Silica
}

\author{
Maurizio Muniz-Miranda, Natale Neto
}

Department of Chemistry, University of Florence, Via della Lastruccia 3, Sesto Fiorentino, I-50019 ITALY

The evolution from simple molecules to complex systems and the origin of life had a determinant step in the peptide formation (Fitz, 2007; Plankensteiner 2005; Bujdak, 2003; Plankensteiner, 2002; Rode, 1999). This occurred in the prebiotic scenario by adsorption of aminoacids on silica, alumina and aluminosilicates, present in prominent amount on the Earth. Clay-catalyzed peptide formation probably involved the condensation reaction of Si$\mathrm{OH}$ groups with the aminoacid carboxyl groups and was favored by hot temperature as well as $\mathrm{NaCl}$ at high concentration (Son, 1998, Bujdak, 1997). Many efforts have been spent to simulate the primitive earth condition that enabled peptide formation, for example, oligopeptides have been obtained from glycine by silica- or alumina-catalyzed dehydration reactions (Rode, 1999; Bujadak, 1999).In the present study the efficiency of silica catalyst is checked by observing the SERS (surface-enhanced Raman scattering) signal of amino acids adsorbed on silver-doped colloidal silica. The SERS technique allows detecting very small amounts of analyte when the reagent is immobilized near metal surfaces constituted by silver, gold or copper nanoparticles. Photoreduction of silver ions has been obtained on silica by visible light, resulting in efficient SERS-active colloidal substrates, with performances comparable to those of the usual silver hydrosols (Muniz-Miranda, 2002). Here, after adsorption of glycine or diglycine on colloidal silica, the irradiation with the 514.5-nm laser line allows the formation of silver clusters and, consequently, the Raman evidence of the adsorbate. Thus, it is possible to detect the peptide formation by observing the SERS spectra of the products deriving from the adsorption of glycine on silica particles. Glycine can be considered one of the most abundant amino acid in the primordial era before the occurring of biosystems, due to its simple structure. It exhibits the strongest reactivity, leading to diglycine and diketopiperazine, the cyclic anhydride of diglicine. 
Bujdak, J. and Rode, B. M. (1997). Silica, alumina, and clay-catalyzed alanine peptide bond formation. J. Mol. Evol. 45:457-466.

Bujdak, J. and Rode, B. M. (1999). Silica, alumina and clay catalyzed peptide bond formation: enhanced efficiency of alumina catalyst. Origins of Life and Evolution of the Biosphere 29:451-461.

Bujdak, J. and Rode, B. M. (2003). Peptide Bond Formation on the Surface of Activated Alumina: Peptide Chain Elongation. Catalysis Letters, 91:149-154.

Fitz, D., Reiner, H. and Rode, B. M. (2007). Chemical evolution toward the origin of life. Pure Applied Chemistry,79:2101-2117.

Muniz-Miranda, M. (2002). SERS effect from silver photoreduced on to silica colloidal nanoparticles. J. Raman Spectroscopy, 33:295-297.

Plankensteiner, K., Reiner, H. and Rode, B. M. (2005). Prebiotic chemistry: The amino acid and peptide world. Current Organic Chemistry, 9:1107-1114.

Plankensteiner, K., Righi, A. and Rode, B. M. (2002). Glycine and Diglycine as Possible Catalytic Factors in the Prebiotic Evolution of Peptides. Origins of Life and Evolution of the Biosphere, 32:225-236.

Rode, B. M. (1999). Peptides and the origin of life. Peptides, 20:773-786.

Rode, B. M., Son, H. L., Suwannachot, Y. and Bujdak, J. (1999). The combination of salt induced peptide formation reaction and clay catalysis: a way to higher peptides under primitive earth conditions, Origins of Life and Evolution of the Biosphere,29:273-286.

Son, H. L., Suwannachot, Y., Bujdak, J. and Rode, B. M. (1998). Salt-induced peptide formation from amino acids in the presence of clays and related catalysts. Inorganica Chimica Acta, 272:89-94.

E-mail: muniz@unifi.it

\section{Chemical Evolution of Biomolecules Induced by Radiation}

\section{Kazumichi Nakagawa}

Graduate school of Human Development and Environment, Kobe University, 3-11 Tsurukabuto, Nada-ku, Kobe 657-8501, Japan

Radiation is believed to make an important role in chemical evolution in space as an energy source from simple inorganic molecules to biomolecules such as amino acids. Since amino acids were detected from some meteorites (Cronin 1997), it is of interest to study the next stage of chemical evolution from amino acid monomers to oligopeptides or peptides. Moreover, through the evolution process, establishment of homochirality is also challenging subject. Here we summarize the achievement of our group on radiationinduced chemical reaction and discuss future problems in study of chemical evolution.

We measured absolute values of absorption cross section of amino acids (glycine, alanine, phenylalanine and methionine) (Kamohara in press) and DNA bases (thymine, guanine) for the photon energy $E$ within $3<E<250 \mathrm{eV}$ using the synchrotron radiation in an attempt to obtain the basic data for radiation effect. Accuracy of absolute values was examined with the Thomas-Reiche-Kuhn sum rule, in which value of integration of the optical oscillator strength distribution $\mathrm{d} f / \mathrm{d} E$ should be equal with the number $N_{\mathrm{e}}$ of total electrons responsible to optical transition within the interest range of photon energy $E$. Value of integrated oscillator strength and the number of electron $N_{\mathrm{e}}$ was 27.3 and 30 for glycine, 31.0 and 36 for alanine, 63.2 and 64 for phenylalanine, and 60.1 and 62 for methionine. Similar results 
were obtained for thymine, value of 47.0 and 48 were obtained. These results show that TRK sum rule is very useful to examine the nature of optical response of biomolecules.

Quantum yield $\phi$ of chemical evolution from amino acid monomers to oligopeptides was determined for soft X-ray (Kaneko 2005, Tanaka 2005) and vacuum ultraviolet. Production of $\mathrm{Gly}_{2}$ and $\mathrm{Gly}_{3}$ was studied for evaporated films of amino acids induced by vacuum ultraviolet (VUV) or X-ray radiation of which photon energy was $E$. Values of $\phi$ were tentatively determined to be: $\phi_{\mathrm{Gly} 1 \rightarrow \mathrm{Gly} 2} \sim 10^{-5}$ and $\phi_{\mathrm{Gly} 2 \rightarrow \mathrm{Gly} 3} \sim 10^{-1}$ for $E \sim 7.2 \mathrm{eV}$. This result shows that the second step $\left(\mathrm{Gly}_{2}\right.$ to $\left.\mathrm{Gly}_{3}\right)$ of this evolution process is easier than the first step (Gly to $\mathrm{Gly}_{2}$ ). Various values of $\phi$ were determined so far for various systems. Magnitude of $\phi$ was around $10^{-3 \pm 2}$, which is not too small.

Since the enantiomeric asymmetry can be introduced through difference in optical absorption coefficient, namely circular dichroism CD, CD spectral data in VUV and X-ray region is inevitable. After achievement of the first CD data at soft X-ray (Tanaka 2005), we developed $\mathrm{CD}$ measurement technique at $3<E<10 \mathrm{eV}$, together with the group of the Advanced Institute of Science and Technology AIST, Tsukuba, Japan (Yamada 2005). Asymmetric decomposition at soft $X$-ray region is now at the focus of attention. Racemization effect of vacuum ultraviolet radiation (Izumi in press) is also discussed.

Cronin, J. R. and Pizzarello, S. (1997). Enantiomeric excesses in meteoritic amino acids. Science 275, 951-955.

Izumi, Y. et al. (in press). Preservation of homochirality of aspartic acid films irradiated with $8.5 \mathrm{eV}$ vacuum ultraviolet light. Radiation Physics and Chemistry.

Kamohara, M. et al. (in press). Optical Oscillator Strength Distribution of Amino Acids from 3 to $250 \mathrm{eV}$ and Examination of the Thomas-Reiche-Kuhn Sum Rule. Radiation Physics and Chemistry.

Kaneko, F. et al. (2005). Chemical evolution of amino acid induced by soft X-ray with Synchrotron Radiation. J. Electron Spectrosc. Relat. Phenom., 144-147, 291-294.

Tanaka, M. et al. (2005). First observation of natural circular dichroism for biomolecules in soft X-ray region studied with a polarizing undulator. Physica Scripta, T115, 873-876.

Tanaka, M. et al. (in press). Fragmentation and dimerization induced by vacuum ultraviolet irradiation. Radiation Physics and Chemistry.

Yamada, T. et al. (2005). VUV circular dichroism spectroscopy using an AC modulated polarizing undulator. Review of Scientific Instruments, 76, 093103.

E-mail: nakagawa@kobe-u.ac.jp

\section{Laboratory Study of Titan's Surface Chemistry Induced by Meteoritic Impact Processing: Laser-Simulated Hypervelocity Impact on ices}

Delphine Nna-Mvondo ${ }^{1}$, Bishun N. Khare ${ }^{2,3}$, Christopher P. McKay ${ }^{2}$

${ }^{1}$ Centro de Astrobiologia (CAB)/CSIC-INTA, Ctra. de Ajalvir, km 4, Torrejon de Ardoz, Madrid, Spain; ${ }^{2}$ NASA Ames Research Center, Moffett Field, CA 94035-1000, USA; ${ }^{3}$ SETI Institute, NASA Ames Research Center, Moffett Field, CA 94035-1000, USA

Titan, Saturn's largest moon, is a planet-size organic reactor where building blocks of life are being generated as they might have been created 4 billion years ago on Earth. Titan's dense 1.5-bar atmosphere, mostly composed of nitrogen and some methane, allows easy 
formation of long chains of organic molecules and high-molecular-weight organic solids, known as tholins. Tholins are aerosols that form a haze in the upper stratosphere of Titan. Over geologic time, both tholins and condensates of the organic gases accumulate in substantial amounts on the surface as liquid and solid. Titan's surface is then a repository of interesting organic molecules generated in the almost complete absence of water but sitting on top of ice. Until recently, researchers have been very careful in their speculations about what might be happening after these molecules get to the surface of Titan. What kind of organic chemistry occurs on the surface? Titan's thick atmosphere protects the surface and organics from harmful cosmic rays and ultraviolet radiation. It has been suggested that these organics could have been subjected to impact processing on Titan's surface (Thompson and Sagan, 1991; Artemevia and Lunine, 2003) and participate in the formation of products relevant to life (Artemevia and Lunine, 2003) such as amino acids, carboxylic acids (Thompson et al., 1992), purines and pyrimidines (Thompson and Sagan, 1991). Subsequent impacts would probably have recycled some of the organic material back into the atmosphere (McKay et al., 1988). Furthermore the presence of condensable agents $\left(\mathrm{C}_{2} \mathrm{~N}_{2}, \mathrm{HCN}\right.$, etc. $)$ along with a natural concentrating mechanism makes polymerization of amino acids or others species likely (Thompson and Sagan, 1991).

Laboratory simulations of meteoritic impact shocks onto Titan's icy surface have not yet been carried out, but preliminary experiments have been performed for planetary icy satellites (Nna-Mvondo et al., 2008). In these previous experiments, the possible chemical production induced by micrometeorite impact shocks on ices has been studied using a highenergy pulsed Nd-YAG laser to reproduce the shock phenomena during hypervelocity micrometeorite impacts into the icy material. The results show the production of various organics and inorganics.

Here we have decided to extend our experiments of laser ablation on ice to a simulated Titan's environment in order to study the effect of meteoritic impacts on the organic chemistry occurring on Titan's surface and to investigate the fate of tholins once condensated into the icy surface and bombarded by meteoritic impacts.

Artemevia, N., and Lunive, J. (2003). Cratering on Titan: impact melt, ejecta, and the fate of surface organics. Icarus, 164: 471-480.

McKay, C.P., Scattergood, T.W., Pollack, J.B., Borucki, W.J., and Van Ghyseghem, H.T. (1988). High-temperature shock formation of $\mathrm{N}_{2}$ and organics on primordial Titan. Nature, 332: 20-522.

Nna-Mvondo, D., Khare, B., Ishihara, T., McKay, C.P. (2008). Experimental impact shock chemistry on planetary icy satellites. Icarus, 194:822-835.

Thompson, W.R., and Sagan, C. (1991). Organic chemistry on Titan-Surface interactions. Proceedings of Symposium on Titan, Toulouse, France, September, 9-12, 1991, ESA SP338, pages $167-176$.

Thompson, W.R., Sagan, C., Stephenson, D., and Wing, M. (1992). Impact-mediated chemical evolution on Titan. Abstract 12.12-P, American Astronomical Society, 24th DPS Meeting, Bulletin of the American Astronomical Society, 24, P.956.

E-mail: nnamvondod@inta.es

\section{ATR-IR Spectroscopic Study of L-Lysine Adsorption on Amorphous Silica Surface}

Norio Kitadai, Tadashi Yokoyama, Satoru Nakashima

Department of Earth and Space Science, Graduate School of Science, Osaka University 
Amino acid adsorption on mineral surfaces has attracted much interest because mineral surfaces may have played an important role in prebiotic peptide bond formation (e.g. Ferris et al., 1996). However, mechanisms of amino acid polymerization reactions on mineral surfaces are poorly understood.

Basiuk and Rode (2001) suggested that acidity or basicity of mineral surfaces can induce changes of the protonation states of amino acid functional groups $\left(\mathrm{NH}_{2}, \mathrm{NH}_{3}{ }^{+}, \mathrm{COOH}\right.$ and $\mathrm{COO}^{-}$), which can enhance the amino acid reactivity. The peptide formation has been found to be greatly affected by the different dissociation states of amino acids with different hydrothermal solution $\mathrm{pH}$ (Zamaraev et al., 1997). Therefore, it is important to quantitatively evaluate the dissociation states of amino acids on mineral surfaces. In this study, attenuated total reflection infrared (ATR-IR) spectroscopy was applied to quantitatively determine the dissociation states of adsorbed L-Lysine on amorphous silica surface.

First, pH-induced ATR-IR spectral changes of dissolved L-Lysine were measured and correlated with thermodynamically calculated dissociation states of Lysine (Di-Cationic, Cationic, and Anionic states). This procedure yielded three calibration lines with good linearity, which can be used for quantitative analysis of adsorbed Lysine on amorphous silica surface.

Two milliliters of $0.2 \mathrm{~mol} / \mathrm{L}$ Lysine solution was first mixed with $500 \mathrm{mg}$ of an amorphous silica gel powder (Wakosil 25SIL). After reaching adsorption equilibrium (about $24 \mathrm{~h}$ ), the suspended solution was placed on an ATR crystal (ZnSe) set in an FT-IR. By subtracting spectra of silica and water, the ATR-IR spectra of adsorbed Lysine on silica surface could be obtained at different $\mathrm{pH}$ from 7.1 to 9.8 .

The obtained ATR-IR spectra of adsorbed Lysine on silica were converted to percentages of four different dissociation states based on the above calibration lines. The results revealed that adsorbed Lysine on amorphous silica surface is present in different dissociation states ( $80 \%$ cationic state and $20 \%$ zwitterionic state) from those in bulk solution. This percentage remain mostly unchanged over the whole tested $\mathrm{pH}=7.19 .8$, while the dissociation states of dissolved Lysine are changing.

ATR-IR spectroscopy is expected to be applied to various amino acids-minerals interactions under different conditions.

Bujdak, J. and Rode, B. M. (2001). Activated alumina as an energy source for peptide bond formation: Consequences for mineral-mediated prebiotic processes. Amino Acids, 21:281291.

Ferris, J. P., Hill Jr, A. R., Liu, R., and Orgel, L. E. (1996). Synthesis of long prebiotic oligomers on mineral surfaces. Nature, 381:59-61.

Zamaraev, K. I., Romannikov, V. N., Salganik, R. I., Wlassoff, W. A., and Khramtsov, V. V. (1997). Modeling of the prebiotic synthesis of oligopeptides: silicate catalysts help to overcome the critical stage. Origins of Life and Evolution of the Biosphere, 27:325-337.

E-mail: nkitadai@ess.sci.osaka-u.ac.jp

\section{Formation and Photo-Stability of Pyrimidine Derivatives from the UV Irradiation of Pyrimidine in Ices}

Michel Nuevo $^{1}$, Stefanie Milam ${ }^{1,2}$, Scott Sandford ${ }^{1}$, Jamie Elsila ${ }^{3}$

${ }^{1}$ NASA Ames Research Center, Moffett Field, CA 94035, USA; ${ }^{2,3}$ NASA Goddard Space Flight Center, Greenbelt, MD 20771, USA 
The detection of amino acids in organic residues formed by the UV photolysis of ices mimicking interstellar and cometary environments $\left(\mathrm{H}_{2} \mathrm{O}, \mathrm{CO}, \mathrm{CO}_{2}, \mathrm{CH}_{3} \mathrm{OH}, \mathrm{NH}_{3}\right.$, etc.) showed that molecules of prebiotic interest can form easily in space (Bernstein et al. 2002; Muñoz Caro et al. 2002). This result agrees with the detection of amino acids in meteorites (Engel and Macko 1997; Cronin and Pizzarello 1997) although their distribution appears to be different (Nuevo et al. 2008), and the (still debated) detection of glycine in molecular clouds (Kuan et al. 2003; Snyder et al. 2005), supporting a scenario where the organic molecules required for life are of extraterrestrial (interstellar or proto-planetary) origin, before being delivered by asteroids, comets, micrometeorites and interstellar dust particles on Earth.

Nucleobases, the building blocks of DNA, constitute another family of prebiotic compounds likely to be formed in space. Larger than amino acids, they are expected to be formed with smaller abundances, and their detection in organic residues requires a specific chemical analytical protocol. Small functionalized polycyclic aromatic hydrocarbons (PAHs), whose structures are close to some of the nucleobases, as well as nucleobases themselves have been detected in meteorites (Stoks and Schwartz 1979; Martins et al. 2004). The formation of nucleobase-like compounds from the UV irradiation of PAHs mixed in ices has been studied in the laboratory (Bernstein et al. 1999, 2001).

In this work, we present a study of the formation of organic compounds from the UV irradiation of pyrimidine at low temperature in ices $\left(\mathrm{H}_{2} \mathrm{O}, \mathrm{NH}_{3}\right)$. Pyrimidine $\left(\mathrm{C}_{4} \mathrm{H}_{4} \mathrm{~N}_{2}\right)$ is the base molecule for three of the five biological nucleobases (cytosine, thymine and uracil), as well as many other derivative compounds. This work aims at studying how pyrimidine is affected by UV photons when it is mixed with precometary ice analogs. In particular, we show how pyrimidine leads to the production of oxidized and amino compounds including nucleobases using high-performance liquid chromatography (HPLC), and study the photo-stability of pyrimidine and its photo-products when subjected to UV photons.

Bernstein, M. P., Sandford, S. A., Allamandola, L. J., Gillette, J. S., Clemett, S. J., and Zare, R. N. (1999). Science, 283:1135-1138.

Bernstein, M. P., Dworkin, J. P., Sandford, S. A., and Allamandola, L. J. (2001). Ultraviolet irradiation of naphthalene in $\mathrm{H}_{2} \mathrm{O}$ ice: Implications for meteorites and biogenesis. Meteor. Plan. Sci., 36:351-358.

Bernstein, M. P., Dworkin, J. P., Sandford, S. A., Cooper, G. W., and Allamandola, L. J. (2002). Racemic amino acids from the ultraviolet photolysis of interstellar ice analogues. Nature, 416:401-403.

Cronin, J. R., and Pizzarello, S. (1997). Enantiomeric excesses in meteoritic amino acids. Science, 275:951-955.

Engel, M. H., and Macko, S. A. (1997). Isotopic evidence for extraterrestrial non-racemic amino acids in the Murchison meteorite. Nature, 389:265-268.

Kuan, Y.-J., Charnley, S. B., Huang, H.-C., Tseng, W.-L., and Kisiel, Z. (2003). Interstellar glycine. Astrophys. J., 593:848-867.

Martins, Z., Botta, O., Sephton, M. A., and Ehrenfreund, P. (2004). Purines and pyrimidines in carbonaceous chondrites: A re-analysis. Meteor. Plan. Sci., 39, Suppl. Proceedings of the 67th Annual Meeting of the Meteoritical Society, August 2-6, 2004, Rio de Janeiro, Brazil, Abstract No. 5145.

Muñoz Caro, G. M., Meierhenrich, U. J., Schutte, W. A., Barbier, B., Arcones Segovia, A., Rosenbauer, H., Thiemann, W. H.-P., Brack, A., and Greenberg, J. M. (2002). Amino acids from ultraviolet irradiation of interstellar ice analogues. Nature, 416:403-406. 
Nuevo, M., Auger, G., Blanot, D., and d'Hendecourt, L. (2008). A detailed study of the amino acids produced from the vacuum UV irradiation of interstellar ice analogs. Orig. Life Evol. Biosph., 38:37-56.

Snyder, L. E., Lovas, F. J., Hollis, J. M., Friedel, D. N., Jewell, P. R., Remijan, A., Ilyushin, V. V., Alekseev, E. A., and Dyubko, S. F. (2005). A rigorous attempt to verify interstellar glycine. Astrophys. J., 619:914-930.

Stoks, P. G., and Schwartz, A. W. (1979). Uracil in Carbonaceous Meteorites. Nature, 282:709-710.

E-mail: mnuevo@arc.nasa.gov

\section{Hypothesis of Formation of Planets from Nebula: Why Are the Planets Different in Their Chemical Compositions?}

Ostrovskii V.E. ${ }^{1}$, Kadyshevich E.A. ${ }^{2}$

${ }^{1}$ Karpov Inst. Phys. Chem., Moscow, Russia; ${ }^{2}$ Obukhov Inst. Atmosph. Phys., Moscow, Russia

Most of the planetists believe that the Solar System originated from a nebula (a giant plasma cloud) (Shmidt, 1949; Hoyle, 1981), which arouse as a result of the supernova explosion about 4.6 billion years ago. More than $99 \%$ of nebular atoms were $\mathrm{H}$ and He. Several models (e.g., Jang-Condell and Boss, 2007; Boss, 2008; Alibert, et al., 2005) were proposed for simulating the processes of planet formation. However, neither the history, nor the physics and chemistry of planet formation are known in detail. There is an opinion that the radius of a planet is the key parameter controlling most of its evolutional features (Albarède and Blichert-Toft, 2007). Meanwhile, a planet radius may be time-dependent and the character of this dependence can not be now specified reliably. The possibility for correlation of models proposed for description of planet formation with the actual transformations of remote stellar systems became available only recently. The evolution causes of the principal differences in the mineral composition and chemical and physical properties of the planets are not yet clarified.

This presentation is an attempt to explain these differences on the basis of a phenomenological model containing new elements. We subdivide the Solar System objects into the physically formed objects (PFO) formed in the cold region of the nebula (from the outside to the present objects of the Main Asteroid Belt) and chemically formed objects (CFO) formed in the hot region of it (Kadyshevich, Ostrovskii, in press). After the big bang, nebula expanded quickly and cooled steadily. In this period, $\mathrm{H}_{2}$ molecules and hydride radicals and molecules with the bond energy exceeding that in $\mathrm{H}_{2}$ (per $\mathrm{H}$ g-atom) formed. With time, nebula transformed to a flat thin disk composed of many concentric diffusely-bounded rings; the more peripheral they were, the lighter molecules they tended to contain. PFO formation started, when the nebula began to collapse after its outer $\mathrm{H}_{2}$ and $\mathrm{He}$ rings cooled to the $\mathrm{H}_{2}$ condensation temperature; $\mathrm{H}_{2}$ droplets absorbed light $\mathrm{Li}, \mathrm{Be}, \mathrm{B}, \mathrm{LiH}$, and $\mathrm{BeH}$ atoms and molecules, which formed the agglomerate cores and increased their size competing with each others for the mass and gravitational attraction. Heavy atoms and hydrides remained in that nebula section in which the temperature was too high for their physical agglomeration and in which their concentration was too low for chemical reactions to proceed to a significant degree. As the nebular-disc compression increased, chemical combination reactions accelerated in the diffusive regions of the neighboring disc rings, exponentially 
stimulated localizations of the substances and reaction heat, and initiated compressible vortexes, within which hot cores of the present sky objects localized. This heat was capable of melting the cores but was not capable of their evaporating. The pressure depletion in the vicinities of the giant vortexes and the gravitational attraction of the last stimulated flows of light cold vaporous and gaseous substances and their asteroid-like agglomerates from the outer space and also of asteroid-like agglomerates of not so light substances from the intermediate regions of the space to the hot cores originated by the vortexes. The flows precipitated over the hot core surfaces of the CFO and cooled these surfaces. The sandwiches obtained as a result of this precipitation became steadily the young Earth-group planets and their satellites. These mechanisms are capable of explaining the planet compositions.

Albarède, F. and Blichert-Toft, J. (2007).Comptes Rendus Geosciences,339(14-15):917-927. Alibert, Y. et al. (2005). Models of giant planet formation with migration and disc evolution. $A \& A, 434: 343-353$.

Boss, A.P. (2008).Flux-limited diffusion approximation models of giant planet formation by disk instability. The Astrophysical Journal,677(1):607-615.

Hoyle, F. (1981). The big bang astronomy. New Scientist, 92:521-527.

Jang-Condell, H. and Boss, A.P. (2007). Signatures of planet formation in gravitationally unstable disks.The Astrophys. J. Letters, 659:L169-L172.

Kadyshevich, E. A. and Ostrovskii V. E. (in press). Planet-system origination and methanehydrate formation and relict atmosphere transformation at the Earth. To appear in Izvestiya, Atmospheric and. Oceanic Physics.

Shmidt, O. Yu. (1949). Four lectures on the Earth-formation theory. Acad. Sci. USSR, M. (Rus.)

E-mail: vostrov@cc.nifhi.ac.ru

\section{Formation of RNA-Oligonucleotides on the Mineral Surface Preliminary Irradiated by UV Light}

Otroshchenko V.A. ${ }^{1}$, Vasilyeva N.V. ${ }^{1}$, Styopina I.E. ${ }^{2}$

${ }^{1}$ A.N. Bach Institute of Biochemistry, Leninsky Prospect 33, Moscow 119072, Russia;

${ }^{2}$ M.V. Lomonosov Moscow State Academy of Subtle Chemical Technology, Moscow, Russia

Probable source of organic molecules is perhaps the surface of mineral particles where the formation of an organic matter occurs which then gets on a surface of planets. The volcanic activity on the ancient Earth, characteristic for many planets, was much more intensive, than now, so it is possible to assume, that in the top layers of an atmosphere owing to volcanic eruptions a plenty of volcanic dust (ashes), clay and gases has been concentrated. The opportunity of biologically significant biopolymers synthesis on a surface of particles of volcanic ashes, clay and $\mathrm{SiO}_{2}$, preliminary irradiated by UV light was studied (the solar spectrum was modeled). The results coincide with earlier obtained upon synthesis of oligonucleotide molecules on a surface of particles of clays or $\mathrm{SiO}_{2}$ : on irradiated by UV mineral surface the biologically important biopolymers (in our caseoligonucleotides) are formed. Now we have shown, that on the surface of particles of the volcanic ashes preliminary irradiated by UV light, there took place the formation of similar polymers from the adsorbed monomers molecules while in the absence of UV irradiation it did not occur. It has been revealed, that upon nucleosides monophosphates adsorption (which generation from water and gas under any energy exposure is possible in relevant conditions) on preliminary irradiated with UV light mineral surface, in some 
cases the formation of linear oligonucleotides occurs. The results, testifying that the amino acids adsorbed on preliminary irradiated mineral surface, also are capable to form polymers (peptides) are received. The assumption of the nature of the molecular mechanism, formed in these conditions biopolymers is put forward. Experimental check of this assumption is spent. Formed linear molecules (in our case-RNA and peptides) could play a corresponding role for evolution and formation of the Earth and prebiological structures.

E-mail: vladotr@inbi.ras.ru

\title{
Energy Sources for Prebiotic Chemistry and Early Life: Constraints and Availability
}

\author{
Robert Pascal, Frédéric Poitevin, Laurent Boiteau
}

Institut des Biomolécules Max Mousseron, UMR5247, CNRS-Université Montpellier 1 \& 2

Following the heterotrophic hypothesis for the origin of life (Oparin, 1924; Haldane, 1929), early living organisms utilized abiotically synthesized organic molecules to build their own components. In addition to this synthetic feature, the energy content carried by these molecules would have been used to maintain their self-organization. It is likely that some of these molecules have constituted the starting material yielding some of the highenergy intermediates (thioesters, acyl phosphates, acyl adenylates, phosphoenol pyruvate, aminoacyl adenylates) that are nowadays involved in the main biochemical pathways. These intermediates are characterized by an energy content corresponding to a range of ca. 30 to more than $60 \mathrm{~kJ} \mathrm{~mol}^{-1}$ per chemical event (hydrolysis for the above mentioned examples). Even in its early stages, the development of the translation machinery required the availability of a source of energy capable of releasing the energy content needed for aminoacid adenylate formation, which is higher than that of ATP by as much as ca. $37 \mathrm{~kJ}$ $\mathrm{mol}^{-1}$ (Wells et al., 1986). Throughout the development of the corresponding processes, carriers capable of releasing energy contents in a similar or upper range have been needed. An assessment of abiotic organic reagents based on the chemistry expected to have taken place on the primitive Earth has been carried out. It includes low-molecular weight activated molecules formed by activation in simulated primitive atmosphere. The results of these investigations will be presented highlighting the possibilities of hydrolytic processes of various precursors including amino acid derivatives such as a-aminonitriles (Lazcano and Miller, 1996) or $\mathrm{N}$-carboxyanhydrides (Pascal et al., 2005). Pathways leading to the utilization of energy are likely to involve downhill chain reactions or protometabolic cycles reminiscent of those found in modern biochemistry. Such stepwise pathways require the presence of chemical energy sources (energy carriers) and the occurrence of coupled reactions for this energy to be distributed to different reaction systems. The requirements for such systems will be analyzed and discussed as well as their consequences for the emergence of protometabolisms trough which life originated and developed (Eschenmoser, 1994; 2007; Pross, 2005, Shapiro, 2006, Commeyras et al., 2004).

Commeyras, A., Taillades, J., Collet, H., Boiteau, L., Vandenabeele-Trambouze, O., Pascal, R., Rousset, A., Garrel, L., Rossi, J.-C., Biron, J.-P., Lagrille, O., Plasson, R., Souaid, E., Danger, G., Selsis, F., Dobrijevic, M., Martin, H. 2004. Dynamic co-evolution of peptides and chemical energetics, a gateway to the emergence of homochirality and the catalytic activity of peptides. Origins Life Evol. Biosphere 34, 35-55.

Eschenmoser, A. 1994. Chemistry of potentially prebiological natural products. Origins Life Evol. Biosphere 24, 389-423. 
Eschenmoser, A. (2007). On a Hypothetical Generational Relationship between HCN and Constituents of the Reductive Citric Acid Cycle. Chem. Biodiversity 4:554573.

Haldane, J. B. S. 1929. The Origin of Life. The Rationalist Annual. Reproduced in: Bernal, J.D., The Origin of Life The Weidenfeld and Nicolson Natural History, R. Carrington, editor, London: Readers Union, 1967, pp. 242-249.

Lazcano, A., Miller, S. L. (1996). The Origin and Early Evolution of Life: Prebiotic Chemistry, the Pre-RNA World, and Time. Cell 85:793-798.

Oparin, A. I. (1924). The Origin of Life. Proiskhodenie Zhini. English translation in: Bernal, J.D., The Origin of Life The Weidenfeld and Nicolson Natural History, R. Carrington, editor, London: Readers Union, 1967, pp. 199-234.

Pascal, R., Boiteau, L., Commeyras, A. (2005). From the Prebiotic Synthesis of $a$-Amino Acids Towards a Primitive Translation Apparatus for the Synthesis of Peptides. Topics in Current Chemistry, 259:69-122.

Pross, A. (2005). Stability in chemistry and biology: Life as a kinetic state of matter. Pure Appl. Chem. 77, 1905-1921.

Shapiro, R. (2006). Small molecule interactions were central to the origin of life. Q. Rev. Biol. 81, 106-125.

Wells, T. N. C., Ho, C. K., Fersht, A. R. (1986). Free Energy of Hydrolysis of Tyrosyl Adenylate and Its Binding to Wild-Type and Engineered Mutant Tyrosyl-tRNA Synthetases. Biochemistry 25, 6603-6608.

E-mail: robert.pascal@univ-montp2.fr

\title{
Molecular Evolution of Clouds Having Varying Initial Composition
}

\author{
Eduardo Monfardini Penteado, Hlio Jaques Rocha-Pinto
}

Observatrio do Valongo/UFRJ

Many molecules important for life are produced and destroyed in interstellar clouds. The collapse of such clouds may originate stars hosting planetary systems. During formation of such systems, molecules of the molecular cloud, aggregated in grains, will be incorporated to the protoplanets, influencing the chemical evolution of the enviroment, maybe favoring the evolution of life at rocky planets located at the stellar habitable zones. Moreover, small bodies, like comets, that hits the formation planet, can carry molecules originated from the molecular cloud. Using astrochemistry equations (Herbst and Klemperer, 1973), we try to describe the evolution of the abundance of that molecules that are important for life from several initial interstellar compositions. These varying initial chemical compositions consider the change of the elemental abundances expected by the Chemical Evolution of the Galaxy (Tinsley, 1980). A system of first order differential equations that describes the varable abundances of each molecule at the gas fase is solved numerically, making possible the knoledge of how the abundance of such molecules change with time and initial chemical composition. We describe preliminary results for how the abundance of many molecules change with time, such as $\mathrm{H}_{2} \mathrm{O}, \mathrm{HCO}, \mathrm{HCN}, \mathrm{NH}_{4}, \mathrm{OH}$ and $\mathrm{CN}$.

Herbst, E. And Klemperer, W., 1973. The Formation and Depletion of Molecules in Dense Interstellar Clouds. ApJ, 1982, 505

Tinsley, B. M., 1980. Evolution of the Stars and Gas in Galaxies. Fund. Cosm. Phys., 5, 287

E-mail: monfpent@ov.ufrj.br 


\title{
Probable Pathways to Prebiotic Carbohydrates and Their Derivates
}

\author{
Oxana Pestunova ${ }^{1,2}$, Alexander Simonov ${ }^{1,2}$, Valentin Parmon ${ }^{1,2}$ \\ ${ }^{1}$ Boreskov Institute of Catalysis; ${ }^{2}$ Novosibirsk State University
}

In this article we summarize and discuss the most significant experimental results on the plausible prebiotic synthesis of carbohydrates and other vitally important organic substances from carbohydrates as initial substrates for such synthesis. Carbohydrates and their derivates play an inestimable role in organic life since they constitute the building blocks of various biomolecules indispensable for the living organisms (DNA, RNA, ATF, cellulose, chitin, starch, etc.). Among carbohydrates the main emphasis is placed on ribose, since the "RNA-world" (Gesteland, 2003) is the most reasoned hypothesis on the prebiotic chemical evolution and origin of life. There are at least two points of view on the origin of first carbohydrates on Earth: (a) carbohydrates were synthesized in the interstellar space at low temperature under action of UV-irradiation or cosmic radiation and were delivered on Earth with comets and meteorites (Finley, 2004); (b) the prebiotic carbohydrates synthesis embodies the catalytic processes in the aqueous solutions of simple substances such as formaldehyde or glycolaldehyde (Pestunova, 2003; Weber, 1995). We support last hypothesis. The synthesis of monosaccharides from formaldehyde and lower carbohydrates (glycolaldehyde, glyceraldehyde, dihydroxyacetone) is catalyzed by different compounds such as natural minerals, phosphate and borate ions (Cairns-Smith, 1972; Pisch, 1995; Simonov, 2007). Ribose can be selectively synthesized from glycolaldehyde and glyceraldehyde in the presence of boratecontaining minerals or Zn-proline complexes (Ricardo, 2004; Ingar, 2003). We demonstrated that lower carbohydrates necessary for the synthesis of monosaccharides can be formed in formaldehyde aqueous solutions under the action of UV-irradiation (Pestunova, 2005). We have shown (Simonov, 2007) that higher monosaccharides can be formed directly from formaldehyde in the course of the combined photochemical and catalytic reactions in plausible prebiotic conditions. Aminoacids and heterocycles can be obtained from carbohydrates and $\mathrm{NH}_{3}$ in the presence of thiols (Weber, 1995).

This research was supported by program of Presidium of RAS Origin and evolution of biosphere, grant RNP.2.1.1.1969 and Integration project of SB RAS 114.

Cairns-Smith, A. G., Ingram, P. and Walker, G. L. (1972) Formose production by minerals: possible relevance to the origin of life. J. Theor. Biol. 35: 601-604.

Finley, D. (2004) Cold Sugar in Space Provides Clue to the Molecular Origin of Life. http:// www.nrao.edu/pr/2004/coldsugar/.

Gesteland, R. F. and Atkins, J. F. editors (1993) The RNA World. Cold Spring Harbor Laboratory, Cold Spring Harbor, New York.

Ingar, A.A., Luke, R.W.A., Hayter, B.R. and Sutherland (2003) Synthesis of cytidine ribonucleotides by stepwise assembly of the heterocycle on a sugar phosphate. Chembiochem: a European journal of chemical biology. 4:504-507.

Pestunova, O., Simonov, A., Snytnikov, V., Stoyanovsky, V. and Parmon, V. (2005) Putative mechanism of the sugar formation on prebiotic Earth initiated by UV-radiation. Adv. Space Res. 36:214-219.

Pisch, S., Eschenmoser, A., Gedulin, B., Hui, S. and Arrhenius, G. (1995) Mineral induced formation of sugar phosphates. Origins of life and evolutions of biosphere. 25: 297.

Ricardo, A., Carrigan, M. A., Olcott, A. N. and Benner, S. A. (2004) Borate minerals stabilize ribose. Science. 303:196. 
Simonov, A. N., Pestunova, O. P., Matvienko, L. G., Snytnikov, V. N., Snytnikova, O. A., Tsentalovich, Yu. P. and Parmon, V. N. (2007) Possible prebiotic synthesis of monosaccharides from formaldehyde in presence of phosphates. Adv. Space Res. 40:1634-1640.

Weber, A. L. (1998) Prebiotic Amino Acid Thioester Synthesis: Thiol-dependent Amino Acid Synthesis form Formose Substrates (Formaldehyde and Glycolaldehyde) and Ammonia. Origins of Life and Evolution of the Biosphere. 28:259-270. and refs therein.

E-mail: oxanap@catalysis.ru

\title{
Emergence of Protometabolisms and the Self-Organization of Non-equilibrium Reaction Networks.
}

\author{
Raphaël Plasson ${ }^{1 *}$, Hugues Bersini ${ }^{2}$, Axel Brandenburg $^{1}$ \\ ${ }^{1}$ Nordita, Stockholm, SWEDEN; ${ }^{2}$ IRIDIA, Brussels, BELGIUM
}

The debate between "Metabolism first" and "Replication first" theories is shaping the discussion about how life originated (Pross, 2004), emphasizing either the necessity of a structured reaction network to maintain information, or the necessity of information to shape the reaction network. In order to solve this apparent paradox, a general approach comes down to understanding how protometabolisms can lead to the emergence of the first template replicators (Shapiro, 2006; de Duve, 2007), from which open-ended evolutive systems can develop (Ruiz-Mirazo et al., 2008).

On the one hand, replication systems must maintain their informational integrity, characterized by a specific topology of the reaction network, implying the necessity of a continuous consumption and use of energy. On the other hand, the presence of a source of free energy should have lead to the self-organization of reaction networks (Plasson and Bersini, submitted), that is to the emergence and maintenance of protometabolisms. Such reservoirs of energy (originating from several external energy sources, like sun light, reduced material from Earth crust, meteorites entering the atmosphere, etc.) generate both linear fluxes of reaction and reaction loops, as attractors of the network (Plasson et al. submitted). This implies the spontaneous generation of network catalysis and autocatalysis, which introduces positive and negative feedbacks inside the system. In such dynamical reaction networks, bifurcation mechanisms leads to the extinction of some pathways, favoring the persistence of other. The topology of the reaction network is subjected to a spontaneous evolution, driven by free energy transfers. Rather than the increase of complexity, this process can be better described as a change in the nature of the complexity, from horizontal complexity (i.e. a large number of simple molecules reacting non-selectively with each other) to vertical complexity (i.e. a large number of complex molecules, built on a limited number of building blocks, engaged in autocatalytic cycles). Such self-organization phenomenon can be linked to an evolution of the "logical depth" as described by Bennett (1986). A model of dynamic polymerization of amino acids will be described as a simple example of such self-organization of reaction network by bifurcation mechanisms (Plasson et al. 2007).

In this scope, the gap separating prebiotic systems from the first reproductive systems can be described as evolutive protometabolisms. The bifurcations, driven by the fighting mechanisms between the network sub-elements, are sources of topological changes inside the reaction networks, from randomness to structures organized around some central compounds. This may have constituted the first replicators, not as template replicators of similar molecules, bu as network replicators of similar reaction cycles, competing with each others. 
Bennett, C. H. (1986). On the nature and origin of complexity in discrete, homogeneous, locally interacting systems. Foundations of Physics, 16:585-592.

de Duve, C. (2007). Chemistry and selection. Chemistry \& Biodiversity, 4:574-583.

Plasson, R. and Bersini, H. (submitted). Energetic and entropic analysis of mirror symmetry breaking process in recycled microreversible chemical system. Submitted to the Journal of Physical Chemistry B. http://arxiv.org/abs/0804.4834.

Plasson, R., Bersini, H. and Brandenburg, A. (submitted). Decomposition of Complex Reaction Networks into Reactons. Submitted to Biophysical Journal. http://arxiv.org/abs/ 0803.1385.

Plasson, R., Kondepudi, D. K., Bersini, H., Commeyras, A. and Asakura, K. (2007). Emergence of homochirality in far-from-equilibrium systems: mechanisms and role in prebiotic chemistry. Chirality, 19:589-600.

Pross, A. (2004). Causation and the origin of life. Metabolism or replication first? Origins of Life and Evolution of the Biosphere, 34:307-421.

Ruiz-Mirazo, K., Umerez, J. and Moreno, A. Enabling conditions for "open-ended evolution" (2008). Biology and Philosophy, 23:67-85.

Shapiro, R. (2006). Small molecule interactions were central to the origin of life. The Quarterly Review of Biology, 81(2):105-125.

E-mail: rplasson@nordita.org

\section{Phosphorylation of Ribose in the Presence of Borate Salts}

\section{Benoît E. PRIEUR}

\section{Ecole Normale Supérieure, Paris}

The discovery of stabilizing properties of borate salts on ribose (Prieur B., 2001, Ricardo et al., 2004) in drastic conditions (high temperature, high $\mathrm{pH}$ ) suggests a pathway of phosphorylation with trimetaphosphate or amidotrimetaphosphate. Furthermore borate salts induce the formation of the furanose cycle (Verchère J.F. and Sauvage J.P., 1988), so it is important to know if borates salts can inhibit phosphorylation of ribofuranose. Halmann and Orgel (1969) phosphorylated D-ribofuranose in the presence of cyanogen or cyanide. High yields of nucleoside phosphates were obtained by Lohrmann and Orgel (1968 and 1971) in solid state reactions with inorganic phosphate. Handschuh and Orgel (1973) showed that the sedimentary mineral struvite, $\left(\mathrm{NH}_{4}\right) \mathrm{MgPO}_{4} \cdot 6 \mathrm{H}_{2} \mathrm{O}$ when heated with urea in the presence of nucleosides, forms nucleoside pyrophosphates in good yield. Furthermore trimetaphosphate has been used in the polyphosphorylation of nucleoside (Schwartz, 1969; Saffhill, 1970; Etaix, E. and Orgel, L. E., 1978; Cheng et al., 2002; Yamagata et al., 1995) nucleotide (Ozawa K. et al., 2004; Yamagata, 1999), glycol (Etaix, E. \& Orgel, L.E., 1978), glycolate (Kolb, V. et al., 1997), glyceric acid (Kolb, V. \& Orgel, L.E., 1996) and amidophosphate in the phosphorylation of glycolaldehyde with high yields (Krishnamurthy, R. \& al., 1999). These observations, when combined together, may suggest a possibility of prebiotic phosphorylation in hydrothermal environments. We will present synthesis of ribose-5-phosphate with the aid of trimetaphosphate and borate salts in a simulated hydrothermal environment.

Cheng, C., Fan, C., Wan, R., Miao, A., Chen, J., and Zhao, Y. (2002) Phosphorylation of Adenosine with Trimetaphosphate under Simulated Prebiotic Conditions, Origins of Life and Evolution of the Biosphere 32, 219-224. 
Etaix, E. and Orgel, L. E. (1978) Phosphorylation of nucleosides in aqueous solution using trimetaphosphate: formation of nucleoside triphosphates, J. Carbohydrates-NucleosidesNucleotides 5, 91-110.

Halmann, M., Sanchez, R. A. and Orgel, L. E. (1969) Phosphorylation of D-ribose in aqueous solution, J. Org. Chem. 34, 3702-3703.

Kolb V., Zhang, S., Xu, Y. and Arrhenius G. (1997) Mineral induced phosphorylation of glycolate ion-a metaphore in chemical evolution, Origins of Life and Evolution of the Biosphere 27, 485-503.

Kolb, V. and Orgel, L. E. (1996) Phosphorylation of Glyceric Acid in Aqueous Solution using Trimetaphosphate, Origins of Life and Evolution of the Biosphere 26, 7-13.

Krishnamurty R., Arrhenius G. and Eschenmoser A. Formation of glycolaldehyde phosphate from glycolaldehyde in aqueous solution. Origins of Life and Evolution of the Biosphere 29: 333-354, 1999.

Lohrmann, R. and Orgel, L. E. (1968) Prebiotic Synthesis: Phosphorylation in Aqueous Solution, Science 161, 64-66.

Lohrmann, R. and Orgel, L. E. (1971) Urea-inorganic phosphate mixtures as prebiotic phosphorylating agents., Science 171, 490-494.

Ozawa K. et al., (2004) Phosphorylation of nucleotide molecules in hydrothermal environments, Origins of Life and Evolution of the Biosphere 34, 465-471.

Prieur B. (2001) Etude de l'activité prébiotique potentielle de l'acide borique, C. R. Acad. Sci., Chemistry, 4, 667-670.

Ricardo A., Carrigan M.A., Olcott A. N. and Benner S.A. (2004) Borate Minerals stabilize Ribose, Science, 303, 196.

Saffhill, R. (1970) Selective phosphorylation of the cis-2',3'-diol of unprotected ribonucleosides with trimetaphosphate in aqueous solution., J. Org. Chem. 35, 2881.

Schwartz, A. W. (1969), Specific phosphorylation of the 2'- and 3'-Position in Ribonucleosides, Chem. Commun., 1393.

Verchère J.F., Sauvage J.P. (1988) A ${ }^{11} \mathrm{~B}$ and ${ }^{13} \mathrm{C}$ NMR determination of the structures of borate complexes of pentoses and related sugars. Tetrahedron. 44 (14), 4469-4482.

Yamagata, Y., Inoue, H. and Inomata, K. (1995) Specific Effect of Magnesium Ion on 2', 3'-Cyclic AMP Synthesis from Adenosine and Trimeta Phosphate in Aqueous Solution, Origins of Life and Evolution of the Biosphere 25, 47-52.

Yamagata, Y. (1999) Prebiotic Formation of ADP and ATP from AMP, Calcium Phosphate and Cyanate in Aqueous Solution, Orig. Life Evol. Biosphere 29, 511-520.

E-mail: prieur7@gmail.com

\section{HCN Black Polymers: A Spectrometric/Spectroscopic Revision}

Ruiz-Bermejo M. ${ }^{1}$, Menor-Salván C. ${ }^{1}$, Rogero C. ${ }^{1}$, Osuna-Esteban S. ${ }^{1}$, Martín-Gago J. A. ${ }^{1,2}$, Veintemillas-Verdaguer S. ${ }^{1,2}$

${ }^{1}$ Centro de Astrobiología (CSIC-INTA); ${ }^{2}$ Instituto de Ciencias de Materiales de Madrid (CSIC)

$\mathrm{HCN}$ is a ubiquitous molecule in the whole Universe and it is a main product in prebiotic simulation experiments (see e.g. Matthews and Minard, 2006, Chen and Chen, 2005, Saladino et al. 2004 and internal references). It has been proposed that the HCN polymers are important substances in the first stages of the chemical evolution to the emergence of life. In a general way, the hydrolysis of these polymers yields purines, pyrimidines, and 
amino acids, as well as of others compounds such as oxalic acid and guanidine (see e.g. Ferris et al. 1973, 1978, Voet and Schwartz 1983). However, in spite of the many efforts made to elucidate their structure and of the proposed models (Umemoto et al. 1987, Ferris et al. 1981, Matthews and Moser 1967 and Völker 1960) some questions are still opened. Since these studies, experimental analytical techniques have advanced enormously. The development of new analytical techniques and the improvement of the resolution of the old ones allow us, nowadays, to solve problems, like the one we are discussing here. The aim of our work is, therefore, to go deeper into the resolution of the unanswered questions and for doing that we have combined many different techniques: FT-IR, CP-MAS ${ }^{13} \mathrm{C}$ NMR, XPS, ESI-TOF, TOF-SIMS and elemental analysis.

It is interesting to point out the use of XPS (X-ray photoelectron spectroscopy) since this technique allows us to identify the elements on the samples as well as the chemical states of these elements. Thus, XPS is very usefull for the unambiguously assignment of nitrogen bonds in the HCN polymers. We found three types of nitrogen chemical environment: $\mathrm{C} \equiv \mathrm{N}, \mathrm{C}=\mathrm{N}$ and $\mathrm{O}=\mathrm{C}-\mathrm{NH}-$. The ESI-TOF mass spectrometry showed fragmentations that indicate an oligomeric structure for the solid. This information, completed with the new results extracted from the other techniques, finally provide new information about the HCN black polymers.

Chen, Q. W. and Chen, C. L. (2005). The role of inorganic compounds in the prebiotic synthesis of organic molecules. Current. Org. Chem. 9, 989-998.

Ferris, J. P., Donner, D. B., Lobo, A. P. (1973). Possible role of hydrogen cyanide in chemical evolution. Investigation on the proposed direct synthesis of peptides from hydrogen cyanide. J. Mol. Evol. 74, 499-508.

Ferris, J. P., Joshi, P. C., Edelson, E. H., Lawless, J. G. (1978). HCN: A plausible source of purines, pyrimidines, and amino acids on the primitive Earth. J. Mol. Evol. 11, 293-311. Ferris, J. P., Edelson, E. H., Auyeung, J. M., Joshi, P. C. (1981). Structural studies on HCN oligomers. J. Mol. Evol. 17, 69-77.

Matthews, C. N., Moser, R. E. (1967). Peptide synthesis from hydrogen cyanide and water. Nature 215, 1230-1234.

Matthews, C. N. and Minard, R. D. (2006). Hydrogen cyanide polymers, comets and the origin of life. Faraday Discuss, 133, 393-401.

Saladino, R., Crestini, C., Costanzo, G., DiMauro, E. (2004) Advances in the prebiotic synthesis of nucleic acids bases: Implications for the origin of life. Current Org. Chem. 8, $1425-1443$.

Umemoto, K., Takahasi, M., Yokota, K. (1987). Studies on the structure of HCN oligomers. Origins of Life 17, 283-293.

Voet, A. B., Schwartz, A. W. (1983). Prebiotic adenine synthesis from HCN-Evidence for a newly discovered major pathway. Biorg. Chem. 12, 8-17.

Völker, T. (1960). Polymere Blausäure. Angew. Chem. 72, 379-384.

E-mail: ruizbm@inta.es

\section{Divalent Metal Ion as a Prebiotic Catalyst for Nucleotidyl Transfer to Form Coenzymes and Ribonucleoitdes Containing Pyrophosphate Bond}

Hiroaki. Sawai

Department of Applied Chemistry and Chemical Biology, Gunma University, Kryuu, Gunma 376-8515 Japan 
We previously reported model reactions of prebiotic synthesis of RNA from nucleoside-5'phjosphorimidazolides $(\mathrm{ImpN})$ by divalent metal ion catalyst such as $\mathrm{UO}_{2} 2+, \mathrm{Pb} 2+$, and $\mathrm{Zn} 2+$ ion. OligoRNAs from 2 mer to 18 mer were formed by the reaction in neutral aqueous solution. The reaction takes places by transfer of ribonucleotidyl group of ImpN to the 2'or 3'-OH group of adjacent molecule of ImpN formiong the phosphodiester bond. Apart from RNA, another group of biologically important compounds consisting of ribonucleotides containing pyrophosphate are prepared by ribonulceotidyl transfer reactions and play essential roles in life. For example, coenzymes such as NAD, FAD and Coenzyme A are involved in the enzymatic oxidation-reduction and acyl transfer reactions, respectively. Sugar-nucleotides such as UDP-glucose are precursors of polysaccharide biosynthesis, and CDP-choline is a precursor of lipid biosy.nthesis. In the present biochemical systems, these nucleotides containing pyrophosphate bond are prepared from NTP and phosphoryl compounds by nucleotidyl transferases that require divalent metal ions such as $\mathrm{Mg} 2+$ ion. Thus, we examined catalytic activity of various divalent metal ions for the nucleotidyl transfer reaction from ImpN and phosphoryl compounds in neutral aqueous solution as a model process of prebiotic synthesis of coenzymes and other biologically important nucleotides containing pyrophsoaphate bond. Among the divalent metal ions examined in our study, $\mathrm{Mn} 2+, \mathrm{Mg} 2+$ and $\mathrm{Cd} 2+$ are most effective catalyst for the nucleotidyl transfer reactions from ImpN and phosphoryl compounds. A number of nucleotide containing pyrophosphate bond, NAD, UDP-glucose, CDP-choline cap portion of mRNA, were prepared by these reactions.

E-mail: sawai@chem.gunma-u.ac.jp

\title{
A Possible New Method for an Abiogenic Synthesis of Pyrimidine Nucleosides and their Acyclic Analogues
}

\author{
Michael B. Simakov
}

Group of Exobiology, Institute of Cytology RAS, Tikhoretsky Av., 4, St.Petersburg, 194064, Russia

There are many unresolved problems in abiogenic synthesis of nucleosides: (1) the absence of a feasible prebiotic pathway to the ribose; (2) the instability of this sugar; (3) the lack of efficient procedures for the synthesis of glycosidic bonds. Therefore alternative genetic macromolecules such as peptide nucleic acids (PNA) and some others have been proposed instead primordial RNA.

We would like to propose a feasible pathway for an abiogenic synthesis of pyrimidine PNA monomers and other nucleoside analogues along with the usual nucleosides. Such acetic acid derivatives as uracil-N'-acetic acid, thymidine $\mathrm{N}^{\prime}$-acetic acid, and cytosine $\mathrm{N}^{\prime}$ acetic acid are readily synthesized in the photochemical reaction of nucleic acid bases (U, T, and C) with the simplest amino acid glycine at the action of UV-light $(\lambda=254 \mathrm{~nm})$ in a water solution with good yields.

The reaction of nucleic acid bases with such amino acid as $\beta$-alanine and $\beta$-or $\gamma$ aminobutyric acids, which are very common in meteorites, also yields a row of the base-N'alkyl acid derivatives. Besides, $\alpha, \gamma$-diaminobutyric acid forms an aspartate-derived nucleoside analogue which could serve as a base monomer for the first genetic material which has similarity with peptides (peptide bond between carboxylic group of one molecule and $\alpha$-amino group of the other) and nucleic acids (heterocyclic bases at $\gamma$-amino groups). 
This type of reaction could also be used for synthesis of such acyclic nucleoside analogues as:

(1) glycerol-derived acyclonucleoside [Base $\left.+\mathrm{H}_{2} \mathrm{~N}-\mathrm{CH}_{2}-\mathrm{CH}_{2}(\mathrm{OH})-\mathrm{CH}_{2}(\mathrm{OH})\right]$, this compound phosphorylated at one or both hydroxyl positions could make a backbone with phosphate bonds;

(2) acrolein-derived nucleoside analogues [Base $+\mathrm{HOCH}_{2} \mathrm{CH}\left(\mathrm{CH}_{2} \mathrm{NH}_{2}\right) \mathrm{CH}_{2} \mathrm{OH}$;

(3) common nucleosides [Base + ribosylamine] (it is an one step process of glicoside bond forming with good yields and great similarity with the processes of the de-novo pyrimidine nucleosides biosynthesis).

So proposed reaction of the photochemical synthesis of alternative genetic monomers in aqueous environments from nucleic acid bases and compounds having amino-group is a plausible pathway for the origin of the pre-RNA world on our planet.

E-mail: exobio@mail.cytspb.rssi.ru

\section{Putative Prebiotic Photocatalytic Synthesis of Monosaccharides in Aqueous Solution of Formaldehyde}

Alexander Simonov ${ }^{1,2}$, Delidovich Irina $^{1,2}$, Oxana Pestunova ${ }^{1,2}$, Valery Snytnikov ${ }^{1,2}$, Valentin Parmon ${ }^{1,2}$

\section{${ }^{1}$ Boreskov Institute of Catalysis; ${ }^{2}$ Novosibirsk State University}

An inestimable role in the organic life is played by carbohydrates. Monosaccharides and their derivates constitute the building blocks of various biomolecules like DNA and RNA, ATF, cellulose, chitin and starch which are indispensable for the living organisms. Among all prebiotic carbohydrates the main emphasis is placed on ribose. Indeed, the RNA-world (Gesteland and Atkins, 1993) is one of the most reasoned hypotheses on the prebiotic chemical evolution and the origin of life. In this work we investigated the possibility of formation of different monosaccharides from the simplest substrate-formaldehyde (hereinafter, FA), in the aqueous solution in possible prebiotic conditions.

We demonstrated that glycolaldehyde (hereinafter, GA) could be formed in aqueous FA solution under the UV-irradiation (Pestunova et al., 2005). From the other hand higher monosaccharides were shown to be synthesized via condensation of formaldehyde and lower carbohydrates catalyzed by phosphates in neutral aqueous solution at mild temperatures. (Simonov et al., 2007). In order to combine these processes an experimental photo-catalytic flow installation was designed.

The starting solution for all experiments contained FA with different concentrations and a catalyst-homogeneous phosphates $\left(\mathrm{Na}_{2} \mathrm{HPO}_{4}+\mathrm{KH}_{2} \mathrm{PO}_{4}\right)$, at $\mathrm{pH}=8$. That is, the sole substrate for the synthesis of monosaccharides was FA known to be an abundant compound of the prebiotic environment. The consecutive photosynthesis of GA and catalytic condensation of FA with lower monosaccharides resulted in the formation of significant amounts of higher monosaccharides. The HPLC analysis of the reaction mixture revealed that erythrulose (tetra-ketose) and 3-pentulose (penta-3-ketose) with maximum yields of $10 \%$ and $5 \%$, respectively, were the major products of the process. At the same time the isomerization of 3-pentulose results in the formation of reasonable amounts of ribulose $(4 \%$ yield). Finally, under the catalytic action of phosphates ribulose is isomerized into ribose and arabinose. The detected concentration of ribose in the reaction mixture was not very 
high. Nevertheless, it is the first evidence of the possibility of the synthesis of these vitally important monosaccharides from FA in putative prebiotic conditions.

In addition to monosaccharides pyruvaldehyde was identified in the reaction mixture. Pyruvic acid was identified in trace amounts. The importance of these compounds for the prebiotic chemistry is discussed in detail in the series of works by Weber (1998).

In conclusion we would like to note that the investigated reactions do not require any complex substrates, extreme conditions and proceed readily in neutral aqueous media. Thus, the combination of the photochemical and catalytic process can be considered as a putative route to the monosaccharides and their derivates on prebiotic Earth.

This research was supported by program of Presidium of RAS Origin and evolution of biosphere, grant RNP.2.1.1.1969 and Integration project of SB RAS 114.

Gesteland R. F. and Atkins J. F. editors (1993) The RNA World. Cold Spring Harbor Laboratory, Cold Spring Harbor, New York.

Pestunova, O., Simonov, A., Snytnikov, V., Stoyanovsky, V. and Parmon, V. (2005) Putative mechanism of the sugar formation on prebiotic Earth initiated by UV-radiation. Adv. Space Res. 36(2):214-219.

Simonov, A. N., Pestunova, O. P., Matvienko, L. G., Snytnikov, V. N., Snytnikova, O. A., Tsentalovich, Yu. P. and Parmon, V. N. (2007) Possible prebiotic synthesis of monosaccharides from formaldehyde in presence of phosphates. Adv. Space Res. 40:1634-1640.

Weber, A. L. (1998) Prebiotic Amino Acid Thioester Synthesis: Thiol-dependent Amino Acid Synthesis form Formose Substrates (Formaldehyde and Glycolaldehyde) and Ammonia. Origins of Life and Evolution of the Biosphere. 28:259-270. and refs therein.

E-mail: san@catalysis.ru

\section{Is the Peptide Bond Formation Activated by $\mathrm{Cu}^{2+}$ Interactions? Insights from Density Functional Calculations}

M. Sodupe ${ }^{1}$, L. Rodríguez-Santiago $^{1}$, A. Rimola $^{2}$, P. Ugliengo $^{2}$

${ }^{1}$ Departament de Química, Universitat Autònoma de Barcelona, Bellaterra 08193, Spain; ${ }^{2}$ Dipartimento di Chimica I.F.M, NIS Centre of Excellence and INSTM National Consortium, Università degli Studi di Torino, via P. Giuria 7-10125 Torino, Italy

Metal cation binding to amino acids and peptides is a very active area of research due to their importance in many fields. With the advent of electrospray ion sources, metal cation complexes of amino acids and peptides can readily be generated in gas phase and studied by mass spectrometry techniques, from which structural and intrinsic reactivity information can be obtained. In particular, low energy collisionally activated dissociation experiments of $\mathrm{Cu}^{2+}$ (Glycine $)_{2}$ show that the $\left[\mathrm{Cu}^{2+}(\text { Glycine })_{2}-\mathrm{H}_{2} \mathrm{O}\right]$ complex, corresponding to the loss of a water molecule, is easily formed, which suggests the occurrence of an intracomplex condensation reaction leading to the formation of a peptide bond between two glycines (Seto and Stone, 1999). This reaction is similar to the Salt Induced Peptide Formation reaction proposed to take place in aqueous solution under prebiotic conditions (Rode, 1999). With the aim of getting a detailed understanding, at the molecular level, of the possible role that $\mathrm{Cu}^{2+}$ may have played in the formation of early peptides in the primitive earth, we have performed a theoretical mechanistic study, both in gas phase and in solution, on the condensation reaction between two glycine molecules in the presence of $\mathrm{Cu}^{2+}$ (Rimola et. al. 2007). Results show that the intracomplex condensation reaction in gas phase is associated to a very high free energy barrier due to the loss of metal coordination during the reaction. However, in 
aqueous solution, the important metal coordination changes observed in gas phase are largely attenuated. Moreover, the synergy between the interaction of glycines with $\mathrm{Cu}^{2+}$ and the presence of water molecules acting as proton-transfer helpers significantly lower the activation, largely favoring the formation of the peptide bond.
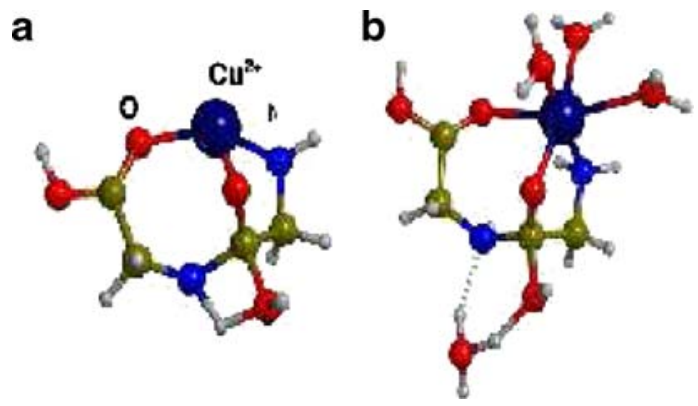

TS structure for the peptide bond formation in a) gas phase and b) aqueous solution.

Rimola Rimola, A., Rodriguez-Santiago, L., Ugliengo, P., Sodupe, M. (2007) Is the Peptide Bond Formation Activated by $\mathrm{Cu}^{2+}$ Interactions? Insights from Density Functional Calculations. J. Phys. Chem. B 111(20): 5740-5747.

Rode, B. M. and Suwannachot, Y. (1999) The possible role of Cu (II) for the origin of life. Coord. Chem. Rev. 190-192:1085-1099.

Seto, C. and Stone, J. (1999) A. Int. J. Mass. Spectrom., 192:289-302

E-mail: mariona.sodupe@uab.es

\section{Experimental Approaches to Fragment Condensation}

Pasquale Stano ${ }^{1}$, Macha Gorlero ${ }^{1}$, Rafal Wieczorek ${ }^{1,2}$, Salvatore Chessari ${ }^{3}$, Pier Luigi Luisi $^{1,3}$

${ }^{1}$ Biology Dept.-University of RomaTre, Rome, Italy; ${ }^{2}$ European Centre for Living Technology (ECLT), Venice, Italy; ${ }^{3}$ Material Dept.- ETH Zurich, Switzerland

It has been proposed that long peptides (or polynucleotides) may form by condensation of shorter sequences, i.e., the so-called fragment-condensation approach [Luisi, 2006]. This mechanism of growth-and-selection may allow the formation of long and possible catalytic biopolymers even in the absence of direct (and/or directed) polymerization reactions.

First, we have experimentally tested this model by combining random peptides (10mers) into an array of 20-mers, and then combining 20-mers into 40-mers. After every elongation step, which was carried out chemically by solid-phase synthesis, only soluble products were used for the next step. In this way, it has been possible to obtain one watersoluble peptide (40-mer) by iterative coupling-selection steps. The final sequence was provided of a short polar segment (four amino acids) at its N-terminus, in order to allow further analysis. Spectroscopic studies indicate the occurrence of stable secondary structure, although the peptide shows no omology with known protein sequences [Chessari et al., 2006].

Secondly, we have investigated the formation of peptide bonds by means of Ser-His, a peptide with esterase and protease activity [ $\mathrm{Li}$ et al., 2000]. By using model compounds, we have demonstrated for the first time that Ser-His succesfully performs reverse-proteolysis 
by combining two peptide fragments, to give new longer peptides [Gorlero et al., submitted]. This proof of principle suggests that short peptides with peptide ligase activity may act as catalysts to originate families of long peptides according to the fragment condensation scenario.

Results are discussed in terms of relevance for the origin of macromolecules.

Chessari, S., Thomas, R. M., Polticelli, F., and Luisi, P. L. (2006) The Production of de novo Folded Proteins by a Stepwise Chain Elongation: A Model for Prebiotic Chemical Evolution of Macromolecular Sequences. Chemistry \& Biodiversity 3, 1202.

Gorlero, M., Wieczorek, R., Stano, P., and Luisi PL (2008) Ser-His catalyzes the formation of peptide bonds. Submitted.

Li, Y., Zhao, Y., Hatfield, S., Wan, R., Zhu, Q., Li, X., McMills, M., Ma, Y., Li, J., Brown, K. L., He, C., Liu, F., and Chen, X. (2000) Dipeptide Ser-His and related oligopeptides cleave DNA, proteins and a carboxyl ester. Bioorg. Med. Chem. 8, 2675.

Luisi, P. L. (2006) The Emergence of Life. From Chemical Origins to Synthetic Biology. Cambridge University Press.

E-mail: stano@uniroma3.it

\section{Active Volcanic Islands as Primordial Molecule Factories}

\section{Henry Strasdeit, Stefan Fox}

Department of Bioinorganic Chemistry, Institute of Chemistry, University of Hohenheim, 70599 Stuttgart, Germany

The first oceans on the young Earth formed in the Hadean eon (4.5-3.8 Ga BP) when the geothermal heat production was considerably higher than today. A plausible assumption is that volcanoes which protruded from the ocean and formed islands were abundant at that time. We hypothesize that active volcanic islands, combined with their local atmospheric and oceanic environment, were exceptional places of chemical evolution. The ideas we present are supported by results from simulation experiments and observations on modern volcanoes.

Volcanic eruptions are frequently accompanied by lightning. This is a well-known phenomenon whose possible prebiotic relevance has been recognized (Navarro-González and Segura, 2004). Volcanic lightning has been observed, for instance, during the birth of the island of Surtsey off the coast of Iceland (Anderson et al., 1965). In present volcanic gases, $\mathrm{H}_{2}$-to- $\mathrm{CO}_{2}$ molar ratios of $0.1-0.5: 1$ are common (Oppenheimer, 2004). Mildly reducing $\mathrm{H}_{2} / \mathrm{CO}_{2} / \mathrm{N}_{2}$ gas mixtures have been shown to produce amino acids when exposed to electrical discharges in the laboratory (Miller, 1998). Moreover, it has recently been demonstrated that amino acid production is also possible by electrical discharges in redoxneutral atmospheres (Plankensteiner et al., 2004; Cleaves et al., 2008). Thus, early volcanic islands may have been locations of abiotic amino acid synthesis.

The evaporation of seawater at hot volcanic coasts, which can still be observed today, produces sea salt crusts that subsequently can experience temperatures up to several hundred degrees Celsius (Edmonds and Gerlach, 2006). We have studied the thermal behavior of amino acids embedded in artificial sea salt and found that between 350 and $550^{\circ} \mathrm{C}$ alkylpyrroles were formed. The alkylpyrroles are sufficiently volatile to escape from places of still higher temperature, where they would otherwise be destroyed.

Solid sea salt releases large amounts of hydrochloric acid when heated (Edmonds and Gerlach, 2006). Laboratory experiments have shown that hydrochloric acid catalyzes the reaction between pyrroles and formaldehyde in aqueous solution. Among the final products 
are dipyrrins (also called dipyrromethanes), which can be thought of as "half-porphyrins". They strongly absorb in the visible region and, in their anionic forms, are versatile redoxactive metal ion chelators (Wood and Thompson, 2007).

In summary, the energy (heat, lightning) and inorganic raw material (atmospheric and volcanic gases, sea salt, water) necessary for the formation of potential photoreceptor and electron-transfer molecules may have been available at a single type of primordial location.

Anderson, R., Björnsson, S., Blanchard, D. C., Gathman, S., Hughes, J., Jónasson, S., Moore, C. B., Survilas, H. J., and Vonnegut, B. (1965). Electricity in volcanic clouds. Science, 148:1179-1189.

Cleaves, H. J., Chalmers, J. H., Lazcano, A., Miller, S. L., and Bada, J. L. (2008). A reassessment of prebiotic organic synthesis in neutral planetary atmospheres. Orig. Life Evol. Biosph., 38:105-115.

Edmonds, M. and Gerlach, T. M. (2006). The airborne lava-seawater interaction plume at Klauea Volcano, Hawai'i. Earth Planet. Sci. Lett., 244:83-96.

Miller, S. L. (1998). The endogenous synthesis of organic compounds. In Brack, A., editor, The Molecular Origins of Life, pages 59-85. Cambridge University Press, Cambridge, UK. Navarro-González, R. and Segura, A. (2004). The possible role of volcanic lightning in chemical evolution. In Seckbach, J., editor, Origins: Genesis, Evolution and Diversity of Life, pages 139-152. Kluwer, Dordrecht.

Oppenheimer, C. (2004). Volcanic degassing. In Rudnick, R. L., editor, Treatise on Geochemistry, Volume 3, pages 123-166. Elsevier-Pergamon, Oxford.

Plankensteiner, K., Reiner, H., Schranz, B., and Rode, B. M. (2004). Prebiotic formation of amino acids in a neutral atmosphere by electrical discharge. Angew. Chem. Int. Ed., 43:1886-1888.

Wood, T. E. and Thompson, A. (2007). Advances in the chemistry of dipyrrins and their complexes. Chem. Rev., 107:1831-1861.

E-mail: h-strasd@uni-hohenheim.de

\section{Nonlinear Increase of Glycyl-Glycyl-Glycine in Solid Glycine Induced by Vacuum Ultraviolet Radiation}

\section{Tanaka, A. Imazu, K. Nakagawa}

Graduate School of Human Development and Environment, Kobe University

Since amino acids were detected from some meteorites (Cronin and Pizzarello, 1997), it is of interest to study the next step of chemical evolution from amino acid monomers to oligopeptides (Kaneko, et al. 2005). In this work we studied process of chemical evolution from glycine (Gly) to glycyl-glycine $\left(\mathrm{Gly}_{2}\right)$ and glycyl-glycyl-glycine $\left(\mathrm{Gly}_{3}\right)$ in solid phase irradiated with vacuum ultraviolet (VUV) light.

We prepared solid-phase film of Gly by the vacuum sublimation technique on the Pyrex glass plate which simulated the surface of space dust or meteorite. No thermal decomposition during sublimation was confirmed by high performance liquid chromatography HPLC measurement. We determined the film thickness to be about $150 \mathrm{~nm}$ to absorb almost all photons for $172 \mathrm{~nm}$ VUV irradiation with $\mathrm{Kr}_{2}$ excimer lamp of which light intensity was estimated to be $4.8 \times 10^{15}$ photons $/ \mathrm{cm}^{2} \mathrm{~s}$ ). We irradiated Gly films in vacuum at room temperature with the irradiation time of $30,60,120,180$, and $240 \mathrm{~s}$. After irradiation, samples were dissolved in distilled water and analyzed with HPLC technique to detect and determine the absolute numbers of $\mathrm{Gly}_{2}$ and $\mathrm{Gly}_{3}$. 
At first the number of produced $\mathrm{Gly}_{2}$ was seen to increase and later began to be saturated and $\mathrm{Gly}_{3}$ was nonlinearly increased. Thus we assumed the two-step reaction model, in which $\mathrm{Gly}_{2}$ was used to produce $\mathrm{Gly}_{3}$.

First, $\mathrm{Gly}_{2}$ is produced by the chemical bond formation between two Gly molecules. The number of produced $\mathrm{Gly}_{2}$ molecules is shown as

$$
N_{\mathrm{Gly} 2}={ }_{1 \rightarrow 2} S I_{0}\left(1-e^{-\mu L}\right) t \ldots
$$

where, $\phi_{1 \rightarrow 2}$ is the quantum efficiency of $\mathrm{Gly}_{2}, S$ the cross section of irradiation sample, $I_{0}$ the light intensity, $\mu$ the absorbing coefficient of Gly at $172 \mathrm{~nm}, L$ the thickness of sample, and $t$ is irradiation time.

Second, Gly ${ }_{3}$ is produced from $\mathrm{Gly}_{2}$ and Gly. The number of produced $\mathrm{Gly}_{3}$ molecules is shown as

$$
N_{\mathrm{Gly} 3}=1 / 4 \phi_{1 \rightarrow 2} \phi_{2 \rightarrow 3} \sigma_{\mathrm{Gly} 2} S I_{0}^{2}\left(1-e^{-2 \mu L}\right) t^{2} \ldots
$$

where, $\phi_{2 \rightarrow 3}$ is the quantum efficiency of $\mathrm{Gly}_{3}$, and $\sigma_{G l y 2}$ is absorption cross section of Gly 2 at $172 \mathrm{~nm}$.

Equation (2) was found to reproduce the experimental results. So we concluded that chemical reaction from Gly to $\mathrm{Gly}_{3}$ is two-step reaction. First $\mathrm{Gly}_{2}$ is produced from two Gly molecules, second $\mathrm{Gly}_{3}$ is produced from Gly $\mathrm{y}_{2}$ and Gly molecules. In the case of $172 \mathrm{~nm}$ VUV irradiation, the value of $1 \rightarrow 22 \rightarrow 3$ was tentatively determined to be $2.49 \times 10^{-5}$ (molecules/photon).

Cronin, J. R. and Pizzarello, S. (1997). Enantiomeric excesses in meteoritic amino acids. Science 275: 951-955

Kaneko, F. et al. (2005). Chemical evolution of amino acid induced by soft X-ray with Synchrotron Radiation. J. Electron Spectrosc. Rel. Phenom, 144-147, 291-294

E-mail: tanaka@radix.h.kobe-u.ac.jp

\title{
Without a Solvent: Self-Assembly of Aromatic Molecules via Solid/Solid Wetting
}

\author{
Frank Trixler ${ }^{1,2}$, Wolfgang M. Heck1 $1^{1,2}$
}

${ }^{1}$ Dept. for Earth and Environmental Sciences, Ludwig-Maximilians-Universität München (LMU) and Center for NanoScience (CeNS), Theresienstrasse 41, 80333 München, Germany; ${ }^{2}$ Deutsches Museum, Museumsinsel 1, 80538 München, Germany

An important topic in the bottom-up approach to the study of the origin of life is the question of which environments and conditions are capable of inducing self-assembly of primordial molecules. Several theories on prebiotic steps towards the origin of life include mineral surfaces in liquid environments. Such solid/liquid interfaces are discussed e.g. in the context of selection, concentration, protection and assembly of organic molecules as well as of catalytic reactions (Hazen, 2005). However, many organic molecules, especially polycyclic aromatic hydrocarbons (PAHs), are virtually insoluble in water. As PAHs and their derivatives are widely discussed in origin of life research as probable primordial compounds (e.g., Ashbourn, et al. 2007), primitive pigments (Mahajan, et al. 2003) and being considered in regard to several functionalities in the PAH world hypothesis (Ehrenfreund, et al. 2006), the question arises of whether mineral surfaces are accessible for self-assembly processes under ambinent conditions for this class of molecules. 
Here we show that PAHs adsorb and self-assemble on mineral surfaces by a process which we term "organic solid/solid wetting" (Trixler, et al. 2007). In this process, PAH nanoparticles - pure or suspended within a matrix - are the direct source of the adsorbate molecules. The behaviour of these solid nanoparticles at the mineral surface can be discussed analogue to a liquid droplet wetting a surface. We exemplify our approach with Anthracene and Pentacene derivatives by presenting results from Scanning Tunneling Microscopy, Molecular Modelling and DFT calculations. Our results demonstrate that a solution of organic molecules is not a general prerequisite for the growth of supramolecular structures on mineral surfaces under ambient conditions.

Ashbourn, S. F. M., Elsila, J. E., Dworkin, J. P., Bernstein, M. P., Sandford, S. A. and Allamandola, L. J. (2007). Ultraviolet photolysis of anthracene in $\mathrm{H}_{2} \mathrm{O}$ interstellar ice analogs: Potential connection to meteoritic organics. Meteoritics \& Planetary Science 42: 2035-2041. Ehrenfreund, P., Rasmussen, S., Cleaves, J. and Chen, L. (2006). Experimentally Tracing the Key Steps in the Origin of Life: The Aromatic World. Astrobiology, 6: 490-520.

Hazen, R. M. (2005). Genesis: Rocks, Minerals, and the Geochemical Origin of Life. Elements 1:135-137.

Mahajan, T. B., Elsila, J. E., Deamer, D. W. and Zare, R. N. (2003). Formation of CarbonCarbon Bonds in the Photochemical Alkylation of Polycyclic Aromatic Hydrocarbons. Origins of Life and Evolution of the Biosphere 33: 17-35.

Trixler, F., Markert, T., Lackinger, M., Jamitzky, F. and Heckl, W.M. (2007). Supramolecular self-assembly initiated by solid-solid wetting. Chemistry-A European Journal, 13: 7785-7790.

E-mail: trixler@1mu.de

\section{Cysteine, Thiourea and Thiocyanate Interaction with Clays: FT-IR and Mössbauer Spectroscopy and X-ray Diffractometry Investigations}

Henrique de Santana ${ }^{1}$, Flávio F. Ivashita ${ }^{2}$, Andrea Paesano Jr. ${ }^{2}$, Ivan G. de Souza $\mathrm{Jr}^{3}$, Antonio C. S. da Costa $^{3}$, Luís O. B. Benetoli ${ }^{1}$, Cristine E. A. Carneiro ${ }^{1}$, Dimas A. M. Zaia ${ }^{1}$

${ }^{1}$ Laboratório de Química Prebiótica, Departamento de Química-CCE, Universidade Estadual de Londrina, 86051-990, Londrina-PR, Brazil; ${ }^{2}$ Departamento de Física-CCE, Universidade Estadual de Maringá, 87020-900, Maringá-PR, Brazil; ${ }^{3}$ Departamento de Agronomia-CCA, Universidade Estadual de Maringá, 87020-900, Maringá-PR, Brazil

The adsorption of cysteine, thiourea and thiocyanate on clays (bentonite, montmorillonite) was studied at different $\mathrm{pH}(3.00,8.00)$. Cysteine, thiourea and thiocyanate were dissolved in seawater, which contains the major elements. More details of the methodology could be found in Benetoli et al. 2007. FT-IR spectra of thiocyanate adsorbed on clays showed small shifts of some bands. The spectra of cysteine and thiourea adsorbed on clays showed that interaction cysteine and thiourea/clays occurs through sulfhydryl and amine groups. In addition, it was shown by Mössbauer spectroscopy that at $\mathrm{pH} 3.00$ cysteine and thiourea did not change signficatively the relative amount of ferric and ferrous ions in the clays. However at $\mathrm{pH} 8.00$ the fraction of ferrous ions in bentonite increased from $8.9 \%$ up to $17.6 \%$ and $21.3 \%$ for thiourea and cysteine, respectively. For montmorillonite this changes from $8.6 \%$ up to $22.3 \%$ for cysteine and up to $16.2 \%$ for thiourea. For thiocyanate, in any of the cases, about $12 \%$ of the iron ions were ferrous, revealing that the reaction did not depend on $\mathrm{pH}$ or the 
clay used. The results are explained considering that the interlayer of clays is very acidic and the HSCN is formed. It is suggested that the HSCN in the interlayer of clays is not reducing ferric ions to ferrous ions ( $\mathrm{Ng}$ and Henry, 1975). Increasing $\mathrm{pH}$ and $\mathrm{Fe}^{2+} / \mathrm{Fe}^{3+}$ ratio in the internal structure of the clay minerals enhance total negative layer charge and thiocompounds affinity to compensate it. The X-ray diffratograms showed that thiocyanate had similar and high preference for the interlayer charge of both clay minerals independent of $\mathrm{pH}$, while thiourea had greater preference for adsorption only at $\mathrm{pH} 8.00$. Cysteine had an ambiguous behavior; it only presents increasing adsorption to the internal interlayer of montmorillonite at $\mathrm{pH} 8.00$.

Benetoli L. O. B., de Souza C. M. D., da Silva K. L., de Souza Jr. I. G., de Santana H., Paesano Jr. A., da Costa A. C. S., Zaia C.T. B. V., Zaia D. A. M. (2007). Amino acid interaction with and adsorption on clays: FT-IR and Mössbauer spectroscopy and X-ray diffractometry investigations. Orig. Life Evol. Biosph. 37: 479-493.

Ng F. T. T. and Henry P. M. (1975). Kinetics and mechanism of the oxidation of thiocyanate by tris(1, 10-phenathroline) iron (III) and its derivates. Canadian J. Chem. 53: 3319-3326.

E-mail: damzaia@uel.br

\section{Adsorption of Adenine on Bentonite and Montmorillonite with and without Preadsorbed Sulfide}

Henrique de Santana ${ }^{1}$, Cláudio M. D. Souza ${ }^{1}$, Diogo R. Janiaski ${ }^{1}$, Cássia Thaïs B. V. Zaia ${ }^{2}$, Dimas A. M. Zaia ${ }^{1}$

${ }^{1}$ Laboratório de Química Prebiótica, Departamento de Química-CCE, Universidade Estadual de Londrina, 86051-990, Londrina-PR, Brazil; ${ }^{2}$ Departamento de Ciéncias Fisiológicas-CCB, Universidade Estadual de Londrina, 86051-990, Londrina-PR, Brazil

Bernal 1951 was the first that suggested that minerals could be played an important role in the adsorption of key monomers from dilute solution and their subsequent condensation to form biopolymers. Besides some doubts about the role of minerals in the processes of selection of monomers (Zaia et al. 2002, Zaia 2004, Benetoli et al. 2007, Benetoli et al. 2008), adsorption of biomolecules on minerals is an important issue in prebiotic chemistry (Lambert, 2008). In the present work, the adsorption of adenine on bentonite and montmorillonite with and without preadsorbed sulfide was studied at different $\mathrm{pH}(2.00,7.00)$. The adenine was dissolved in seawater at concentrations of $600,1,200,2,400$ and $3,600 \mu \mathrm{g} 5 \mathrm{~mL}^{-1}$. All clays were processed as follow: to five different sets of four tubes $(15 \mathrm{~mL})$ containing $500 \mathrm{mg}$ of clay (with or without sulfide preadsorbed) were added: (a) $5.00 \mathrm{~mL}$ of seawater, (b) $5.00 \mathrm{~mL}$ of seawater with $120 \mu \mathrm{g} \mathrm{mL}^{-1}$, (c) $5.00 \mathrm{~mL}$ of seawater with $240 \mu \mathrm{g} \mathrm{mL}{ }^{-1}$, (d) $5.00 \mathrm{~mL}$ of seawater with $480 \mu \mathrm{g} \mathrm{mL}^{-1}$ and (e) $5.00 \mathrm{~mL}$ of seawater with $720 \mu \mathrm{g} \mathrm{mL}^{-1}$. The $\mathrm{pH}$ was adjusted to 2.00 or 7.00 with $\mathrm{HCl}$ or $\mathrm{NaOH}$. The tubes were mixed for $4 \mathrm{~h}$, after they were spun for $15 \mathrm{~min}$ at 2,000 rpm; the aqueous phase was used for the adenine analysis (UV $260 \mathrm{~nm})$. All results are presented as mean \pm standard error of mean, and the number of experiments was always five with four sets each. For montmorillonite the following results of adenine adsorbed were obtained: $\mathrm{pH} 2.00$ [without sulfide $291.0 \pm 10.6,821.0 \pm 4.0,1382.6 \pm$ $10.1,1600.5 \pm 16.6$; with sulfide $379.5 \pm 11.4,929.5 \pm 19.9,1625.0 \pm 31.5,1890.2 \pm 31.1]$ and $\mathrm{pH} 7.00$ [without sulfide $269.9 \pm 12.9,583.6 \pm 14.5,911.3 \pm 9.0,1048.5 \pm 18.3$; with sulfide $143.5 \pm 15.6,224.6 \pm 29.8,434.2 \pm 14.9,612.5 \pm 20.4]$. For bentonite the following results of 
adenine adsorbed were obtained: $\mathrm{pH} 2.00$ [without sulfide $411.2 \pm 14.7,773.8 \pm 24.1,1,108.8 \pm$ $6.5,1,387.9 \pm 17.4$; with sulfide $405.7 \pm 17.4,808.5 \pm 19.5,1,149.4 \pm 19.3,1,402.8 \pm 25.2]$ and $\mathrm{pH} 7.00$ [without sulfide $174.6 \pm 7.2,296.2 \pm 7.3,459.7 \pm 10.7,548.9 \pm 16.9$; with sulfide $62.7 \pm$ $10.7,103.6 \pm 10.1,120.6 \pm 20.0,247.2 \pm 8.3]$. For all samples adenine was more adsorbed at $\mathrm{pH} 2.00$ than $\mathrm{pH} 7.00$. At $\mathrm{pH} 2.00$ bentonite and montmorillonite are negatively charged and adenine is positively charged and at pH 7.00 adenine is neutral (Benetoli et al. 2008). Thus the difference of charges clays/adenine could explain why adenine is more adsorbed at $\mathrm{pH} 2.00$ than at $\mathrm{pH}$ 7.00. Sulfide increased the adsorption of adenine at $\mathrm{pH} 2.00$ when compared to the samples without it, by the other hand decreased the adsorption at $\mathrm{pH} 7.00$. These results are now under analysis by FT-IR and Mössbauer spectroscopy.

Benetoli L. O. B., de Santana H., Zaia C.T. B. V., Zaia D. A. M. (2008). Adsorption of nucleic acid bases on clays: an investigation using Langmuir and Freundlich isotherms and FT-IR spectroscopy. Monatshefte für Chemie DOI 10.1007/s00706-0080862-z.

Benetoli L. O. B., de Souza C. M. D., da Silva K. L., de Souza Jr. I. G., de Santana H., Paesano Jr. A., da Costa A. C. S., Zaia C.T. B. V., Zaia D. A. M. (2007). Amino acid interaction with and adsorption on clays: FT-IR and Mössbauer spectroscopy and X-ray diffractometry investigations. Orig. Life Evol. Biosph. 37: 479-493.

Bernal J. D. (1951). The physical basis of life. Routledge and Kegan Paul, London.

Lambert J. F. (2008). Adsorption and Polymerization of Amino Acids on Mineral Surfaces: A Review. Orig. Life Evol. Biosph. DOI 10.1007/s11084-008-9128-3

Zaia D. A. M. (2004). A review of adsorption of amino acids on minerals: was it important for origin of life? Amino Acids 27: 113-118.

Zaia D. A. M., Vieira H. J., Zaia C. T. B. V. (2002). Adsorption of L-amino acids on sea sand. J. Braz. Chem. Soc. 13: 679-681.

E-mail: damzaia@uel.br 


\title{
Origins of Genetic Information
}

\section{A Primitive RNA Transition Scenario Without Cytosine and with Peptides Interacting with RNA: Implications for the Origin of the Genetic Code}

\author{
${ }^{1}$ Delaye L., ${ }^{1}$ Becerra A., ${ }^{2}$ Martinez-Mekler G., ${ }^{3}$ Cocho G. \\ ${ }^{1}$ Laboratorio de Microbiología, Facultad de Ciencias, UNAM, Mexico D.F. 04510, Mexico; \\ ${ }^{2}$ Centro de Física, UNAM, Cuernavaca, 62251, Mexico; ${ }^{3}$ Instituto de Física, UNAM, \\ Mexico D.F. 01000, Mexico
}

We propose a primitive RNA transition scenario without cytosine and with peptides interacting with RNA. We consider riboproteins as representative of these primitive peptides and compute these amino acid frequencies. The more frequent amino acids found are: Lys, Ala, Val, Arg, Leu, Gly, Ile and Glu. In addition to glycine, amino acids with helix propensities dominate. These more frequent amino acids can be coded by uracyl, adenine and guanine, without cytocine, and by NNR codons. The analysis suggest a primitive genetic code with RRR for polar amino acids (gly, glu, lys and arg) and YYR, YRR and RYR for non polar ones and stop codons. Later, with cytosine arrival serine, proline, threonine and glutamine would be coded by NNR codons containing cytosine, and perhaps much later, NNY codons would be occupied by additional low frequency amino acids. Previous, old, amino acids would also occupy the new NNY codons.

E-mail: cocho@fisica.unam.mx

\section{Amino Acid Homochirality Based on the Origin of Phosphate-Based Life}

\section{Daxiong $\operatorname{Han}^{1}$, Haiyan Wang ${ }^{2}$, Yufen Zhao ${ }^{1,3}$}

${ }^{1}$ Department of Pharmacy, Medical College of Xiamen University, Xiamen, China; ${ }^{2}$ Third Institute of Oceanography, State Oceanic Administration of China, Xiamen, China; ${ }^{3}$ The Key Laboratory for Chemical Biology of Fujian Province, College of Chemistry and Chemical Engineering, Xiamen University, Xiamen, China

The emergence of phosphorylation has to have been one of the key events in prebiotic evolution on earth. In this paper, the emergence of phosphoryl amino-acid 5'nucleosides having a $\mathrm{P}-\mathrm{N}$ bond is described as a model of the origin of amino-acid homochirality and genetic code (Figure 1). It is proposed that the intramolecular interaction between the nucleotide base and the amino-acid side-chain influences the stability of particular amino-acid 5'-nucleotides, and the interaction also selects for the chirality of amino-acids. The differences between L- and D-conformation energies $\left(\Delta E^{\text {conf }}\right)$ are evaluated by DFT methods at the B3LYP/6-31G(d) level. Although, as expected, these $\Delta E^{\text {conf }}$ values are not large, they do give differences in energy that can distinguish the chirality of amino-acids. Based on our calculations, the chiral selection of the earliest amino-acids for L-enantiomers seems to be determined by a clear stereochemical /physicochemical relationship. As later amino-acids developed from the earliest amino-acids, we deduce that the chirality of these late amino-acids was inherited from that of the early amino-acids. This idea reaches far back into evolution, and we hope that it will guide further experiments in this area. 


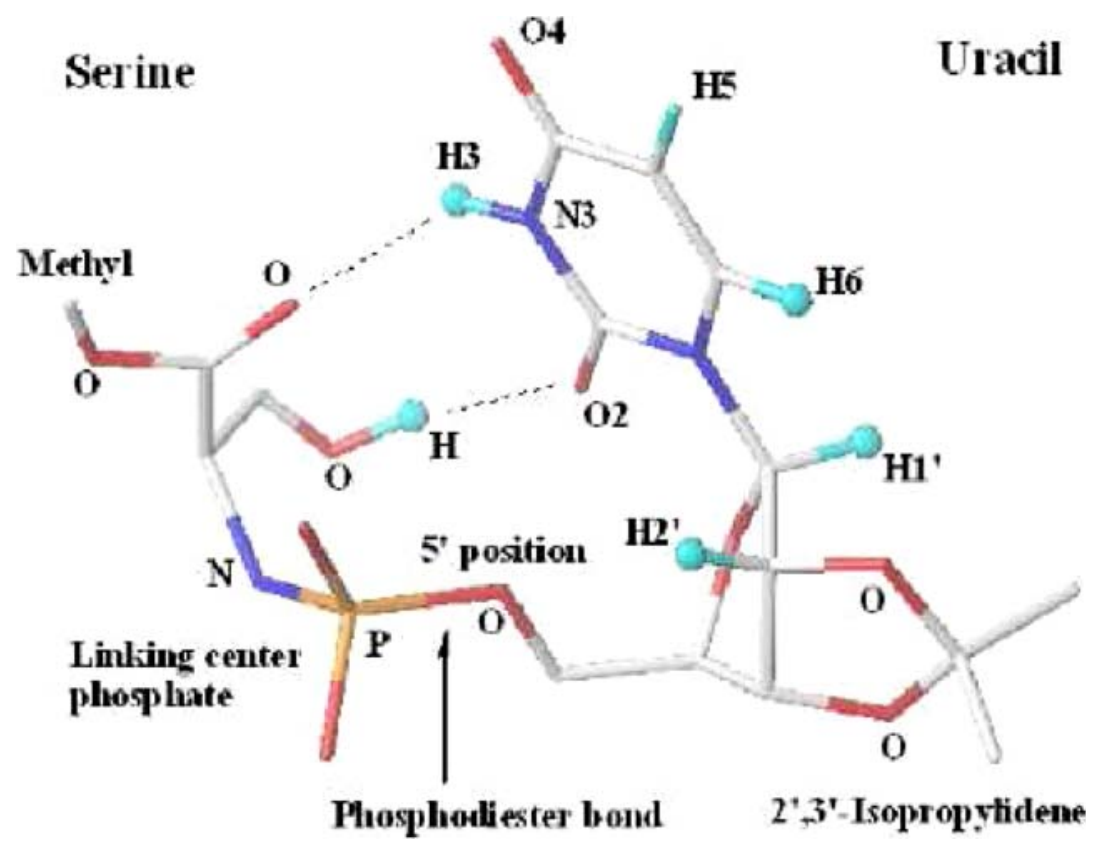

Figure 1. The structure model of the (N)amino acid-5'-nucleoside (dashed line stands for H-bond)

Arrhenius, G., Sales, B., Mojzsis, S., and Lee, T. (1997). Entropy and charge in molecular evolution: the role of phosphate. The Journal of Theoretical Biology 187: 503-522.

Bonner, W.A. (2000). Parity violation and the evolution of biomolecular homochirality. Chirality, 12: 114-126. Jorissen, A., and Cerf, C. (2002). Photoreactions as the Origin of Biomolecular Homochirality: A critical review. Origins of Life and Evolution of the Biosphere, 32: 129-142.

Cheng, C.M., Fan, C., Wan, R., Tong, C.Y., Miao, Z.W., Chen, J., and Zhao, Y.F. (2002). Phosphorylation of adenosine with trimetaphosphate under simulated prebiotic condition. Origins of Life and Evolution of the Biosphere, 32:219-224.

Di Giulio, M. (2004). The coevolution theory of the origin of the genetic code. Physics of Life Reviews, 2: 128-137.

Yang, P., and Han, D.X. (2000). Molecular modeling of the binding mode of chiral metal complex $\Delta$-and $\Lambda$-[Co(phen)2dppz]3 + with DNA. Science in China B, 43: 516-523.

E-mail: daxiong@xmu.edu.cn

\section{N-phosphoryl Amino Acids Reacted with Mixture of Four Nucleosides} (A, G, C and U) in Aqueous Solution: A Clue for Genetic Code Origin

Hongxia Liu ${ }^{1}$, Xiang $\mathrm{Gao}^{2}$, Yibao $\mathrm{Jin}^{1}$, Hui $\mathrm{Li}^{1}$, Yuyang Jiang ${ }^{1 *}$, Yufen $\mathrm{Zhao}^{2 *}$

${ }^{1}$ The Key Laboratory of Chemical Biology, Guangdong Province, Graduate School of Shenzhen, Tsinghua University, Shenzhen, 518057, P. R. China; ${ }^{2}$ Department of Chemistry and The Key Laboratory for Chemical Biology of Fujian Province, College of Chemistry and Chemical Engineering, Xiamen University, Xiamen, 361005, P. R. China 
N-phosphoryl amino acids are unique chemical species with many novel properties, for instance, the ability to self-assemble into oligopeptides in aqueous solution. In our previous work, N- (O, O-diisopropyl) phosphoryl threonine could react with uridine to form peptides and nucleotides in anhydrous pyridine. So Zhao et al. proposed a hypothesis that interaction of N-phosphoryl amino acids with nucleosides could be considered as a model for coevolution of proteins and nucleic acids (Zhou, et al. 1996; Zhao and Cao, 1994; Zhao and Cao, 1999; Zhao, et al. 2000). Therefore, the reaction of N-phosphoryl amino acids with nucleosides could be regarded as a model to study many processes of life origin, such as genetic code origin and chiral origin. In this work, the reactions of N-phosphoryl amino acids (Contained old amino acids) and mixture of four nucleosides (A, G, C, U) in aqueous solution were investigated by UPLC-HRMS and ${ }^{31} \mathrm{P}$ NMR. It was found that the amounts and kinds of dinucleotides formed by the reaction depended on specific N-phosphoryl amino acids and nucleosides. For example, N- (O, O-diisopropyl) phosphoryl alanine prefered to form $\mathrm{CpG}$ (or $\mathrm{GpC}$ ). However, UpA was very difficult to be formed for most of the N-phosphoryl amino acids. The results provide some possible clue to the origin and chemical evolution of genetic code in the prebiotic process.

Zhou W. H., Ju Y., Zhao Y. F. (1996). Origins Life Evol. Biosphere, 26:547.

Zhao Y. F., Cao P. S. (1994). J. Biol. Phys., 20:283.

Zhao Y. F., Cao P. S. (1999). Pure Appl. Chem., 71:1163.

Zhao Y. F., Hu J. J., Ju Y. (2000). Chin. Chem. Lett., 11 (5):407.

E-mail: liuhx@sz.tsinghua.edu.cn

\section{A Conformational Effect of the DNA Double Helix Isotopy: Key to the Molecular-Biological Evolution of Nature}

Andrey A. Ivanov ${ }^{1}$, Vyacheslav S. Sevastianov ${ }^{1}$, Vyacheslav V. Perfilov ${ }^{2}$, Aleksander G. Letuchev $^{2}$

\footnotetext{
${ }^{1}$ Vernadsky Institute of Geochemistry and Analytical chemistry; ${ }^{2}$ Moscow physicalengineering University
}

As it has been reported (Ivanov and Galimov, 2007, Ivanov and Sevastyanov, 2006, Ivanov, 2007, Ivanov, 2007 and Ivanov, 2003), the DNA isotope does make an impact on its own double helical conformational system status according to the appropriate molecular biology tests. An essential meaning of the regularity revealed derives from a known interdependence between the DNA conformational status and the expression of genes (Zhizhina, et al. 2001). In the light of the latter, the DNA double-helix system is nothing but a multidimensional and biologically universal multifunctional interface possessing a capability to record, transmit, store and transform both chemical and physical signals originated by the surrounding atomic/ molecular environment. Apparently, this is a kind of linker between the living objects and inorganic matter; an understanding of that would make clear a mechanism of control over the genome expression during the adaptation towards a renovated environmental conditions. These adaptation moves are to be fixed up in conformation with a subsequent transmission and transformation due to the DNA isotopy specificity.

A meaning of the effect revealed is all about the following. A non-proportional distribution of the isotropically different nucleotide forms within a pair of the double-helix chains caused by an inequality of their physical/chemical properties leads to the isotopyrelated dependence of a whole system, i.e. an isotopy-conformation dependence. This 
dependence is found to be a true regularity being proven in experiments. The above mentioned references include a description of examples of the isotopy-different nucleotide non-proportional distribution.

The Evolution Biology, as long as its taken with a respect to the very nature of molecular mechanisms discussed does not involve yet any applied studies that would deal directly with a regularity we found. That's why the reason for our present work is to attract the attention of academic community to these and related studies. For deeper understanding of both mechanisms and evolutionary significance of the unique phenomenon discovered, a further extensive research required.

We do appreciate R.A.S. Academicians E. M. Galimov and A. L. Buchachenko as well as Prof. Dr. D. A. Kuznetsov for their permanent attention to this work, moral support and advisory assistance.

Ivanov, A. and Galimov, E. (2007). Molecular isotopy of conformational interactions. Symposium on isotopic geochemistry named by A. Vinogradov, pages 44-45. Moscow, Russia. Ivanov, A. and Sevastyanov, V. (2006). Study of the free nucleotides pool $\delta^{13} \mathrm{C}$ changes in polymerase chain reactions. International Congress on Analytical Science ICAS-2006, page 489. Moscow, Russia.

Ivanov, A. (2006). A forced conformational polymorphism of the blastomer DNA. International Congress on Analytical Science ICAS-2006, page 116. Moscow, Russia.

Ivanov, A. (2007). Does the conformation of DNA depend on the difference in the isotope composition of it's threads. Russian Journal of Physical Chemistry, 6(1).

Ivanov, A. (2003). isotope-ragulator of metabolism. GoldshmidtConference, page A180.

Zhizhina, G, Skalatskaya, S. et al. (2001). V.46. [2116]2, 341 pages.

E-mail: aiva@geokhi.ru

\title{
Evolution of Bacterial Regulatory Networks:"The Role of DNA-Binding Specificity"
}

\author{
Irma Lozada-Chvez ${ }^{1}$, Bruno Contreras-Moreira ${ }^{1,2}$, Julio Collado-Vides ${ }^{1}$
}

${ }^{1}$ Centro de Ciencias Genmicas, Universidad Nacional Autnoma de Mexico, Apdo. Postal 565-A, Av. Universidad, Cuernavaca, Morelos, 62100. Mexico; ${ }^{2}$ Estacin Experimental de Aula Dei, Consejo Superior de Investigaciones Cientficas, Av. Montaana 1.005. 50059. Zaragoza, Spain

Over millions of years, both bacterial genome and their gene regulation have changed extensively, structured and adapted to occupy virtually every environmental niche on Earth. In particular, transcriptional regulation (TR) has provided one of the three major evolutionary steps, whereby gene expression and natural variation occurs in biological species. Transcriptional regulation in prokaryotes depends generally upon the recognition of specific DNA operator sites (bsDNA) by transcription factors (TFs). These proteinDNA interactions conforming transcriptional regulatory networks (TRNs) affect the synthesis of messenger RNA molecules of target genes (TG), which can be activated or repressed. It has been demonstrated that TFs have a differential connectivity, in which a small set of TFs regulates a much larger set of TGs (global regulators); whilst a large proportion of TFs in a network affect the expression of only one or few genes (local regulators) (Consentino et al., 2007). The evolutionary reasons that maintain this structure have remained unknown given that TRNs are poorly conserved across bacterial species and global regulators do not necessarily share similar evolutionary histories nor 
necessarily regulate similar metabolic responses in different organisms (Lozada-Chvez et al., 2006).

Here, we analyze this issue through different genomic and bioinformatics approaches using experimental and compiled data of TFs and their bsDNAs from Escherichia coli and Bacillus subtilis, the two best known prokaryotic TRNs with remarkably different niches and evolutionary histories (Lozada-Chvez et al., 2006). We found that paralogy relationships are insufficient to explain the global or local role observed for TFs within regulatory networks, as previously reported (Consentino et al., 2007). Our results provide a picture in which DNA-binding specificity, a molecular property defined here as the ability of DNAbinding proteins (TFs) to discriminate a small subset of DNA sequences from the vast repertoire of sequences found in a genome, is a predictor of the role of TFs. In particular, we observed that global regulators consistently display low levels of binding specificity, while displaying comparatively higher expression values in microarray experiments. In addition, we found a strong negative correlation between binding specificity and the number of co-regulators that help to coordinate genetic expression on a genomic scale. A close look at several orthologous TFs, including FNR, a regulator found to be global in $E$. coli and local in B. subtilis, confirms the diagnostic value of specificity in order to understand their regulatory function, and highlights the importance of evaluating the metabolic and ecological relevance of effectors as another variable in the evolutionary equation of regulatory networks. Finally, a general model that integrates some evolutionary forces and molecular properties is presented, aiming to explain how regulatory modules (regulons) grow and shrink, as bacteria have tuned their regulation to increase adaptation from their Early Evolution to the current Life.

Cosentino Lagomarsino, M., Jona, P., Bassetti, B. and Isambert, H. (2007). Hierarchy and feedback in the evolution of the Escherichia coli transcription network. Proceedings of the National Academy of Sciences USA, 104: 5516-5520.

Lozada-Chavez, I., Janga, S. C. and Collado-Vides, J. (2006). Bacterial regulatory networks are extremely flexible in evolution. Nucleic Acids Research, 34: 3434-3445.

E-mail: ilozada@ccg.unam.mx

\title{
Theoretical Study of the Adsorption of RNA Bases on a Surface of $\mathrm{Na}^{+}$-Montmorillonite
}

\author{
Pierre Mignon $^{1}$, Piero Ugliengo ${ }^{2}$, Mariona Sodupe ${ }^{1}$
}

${ }^{1}$ Universitat Autònoma de Barcelona, Dep. Quimica, 08193 Bellaterra, Spain; ${ }^{2}$ University of Torino, Dip. Chimica IFM, Via P. Giuria, 7. I-10125 Torino-Italy

In the genetic-first viewpoint of the origin of Life, the RNA molecule is proposed to play the role of protein and DNA, i.e. catalyze reactions and store the genetic information essential for life to begin the long path to cellular evolution. The RNA prebiotic synthesis remained, however, a problem that has been tackled for several years by Ferris et al. (Ferris, 2005). His idea is based on clay mineral catalysis of RNA, because clays and in particular Montmorillonite deposits, largely found in volcanic ash, can adsorb organic molecules and prevent them from decomposition. Moreover clay minerals are known for their ability to catalyze organic reactions through the action of bound metal cations. In that aim Ferris investigated the oligomerization of RNA on Montmorillonite for different conditions by using activating groups and mono-metal exchanged $\mathrm{Na}^{+}$-Montmorillonite that gave up to 
50-mers in one day. Following these studies our goal is to shed light on the mechanism of the formation of the phosphodiester bond catalyzed by $\mathrm{Na}^{+}$-montmorillonite by using Quantum Chemical methods. As a starting point we investigated the adsorption of RNA bases on a surface of $\mathrm{Na}^{+}$-montmorillonite. Periodic plane wave DFT calculations were performed with VASP (Kresse, 1993) on an Ottay type montmorillonite model. The cell consists of 2 unit cells of pyrophilite where one $\mathrm{Al}^{3+}$ is substituted by $\mathrm{Mg}^{2+}$. The negative charge is compensated by $\mathrm{Na}^{+}$adsorbed on the surface of the clay. The optimized structure is then used to investigate the adsorption modes of nucleobases. Adenine, Cytosine, Guanine, Uridine and Thymine were optimized in different configurations, considering the interaction via the nitrogen and/or oxygen hetero atoms, the $\mathrm{Na}^{+} /$pi interaction, the direct interaction with the surface, and no interaction with the surface. For each optimized structure we discuss the role of the cation and the role of the surface on the energies, and geometric parameters. The Grimme correction describing the dispersion contribution (Grimme, 2006) to the energy is included to the final energy of adsorption, which allows us to discuss the effect of the Van Der Waals forces. This study follows previous works on the role of mineral material in prebiotic chemistry, in particular the formation and catalysis of the peptide bond by aluminosilicate surface (Rimola, 2007).
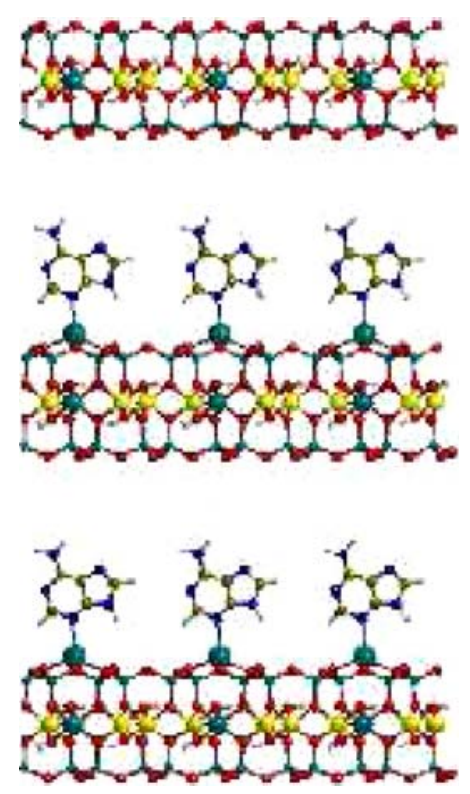

Ferris, J. P. (2005). Mineral catalysis and prebiotic synthesis: Montmorillonite-catalyzed formation of RNA. Elements, 1:145-149.

Grimme, S. (2006). Semiempirical GGA-type density functional constructed with a longrange dispersion correction. J. Comput. Chem., 27:1787-1799.

Kresse, G., and Hafner, J. (1993). Ab initio molecular dynamics for open-shell transition metals. Phys. Rev. B: Condens. Matter Mater. Phys., 48:13115-13118.

Rimola, A., Sodupe, M., and Ugliengo, P. (2007). Aluminosilicate surfaces as promoters for peptide bond formation: An assessment of Bernal's hypothesis by ab initio methods. J. Am. Chem. Soc., 129:8333-8344.

E-mail: pierre@klingon.uab.es 


\section{The Genetic Code: A Primordial "Lingua Cosmica"}

\section{Vladimir Cherbak}

Department of Mathematics, al-Farabi Kazakh National University71 al-Farabi Avenue, Almaty 050038, The Republic of Kazakhstan

A "Lingua Cosmica" (LINCOS) was proposed by Freudenthal (1960) for interstellar communications. He proceeded on the reasonable assumption that arithmetic and its numerical language are the same the universe over. The history of terrestrial mathematics confirms his assumption quite well. Therefore, a preamble of any message should be arithmetical to be easily understood by an intellectual addressee. Needless to say, the natural series as well as examples of arithmetical operations should be presented first of all. Freudenthal used for that the so-called "ostensive numerals", i.e. certain sets of identical radio pulses or "beeps". He accompanied these numerals with their dyadic notations.

Dutil and Dumas (2003) improved Freudenthal's pattern for a real broadcast. They supplemented those dyadic notations with the decimal ones. The decimals, among other things, show the artificial origin of the broadcast itself. Indeed, the place-valued decimal system with zero conception is an indisputable artifact of the mind. Some signs of our knowledge have been broadcast, too. These are the "Egyptian triangle", the zero sign at the beginning of the natural series, and a structure of DNA. The radio telescope broadcast toward five stars took place in Evpatoria, Ukraine and Roswell, New Mexico, U.S.A. on July 6th 2003.

Admittedly, the genetic code - a kingpin of the life information system-holds the key to a mystery of the origin of life. The first thing for a new molecular biology is its strict scrutiny. Therefore, the genetic code itself should be the best place for the preamble, if there were a genetic channel for an intellectual message. Though the following words stagger belief, it seems that such channel exists. The simple and uniform grammar discloses a primordial message incorporated into the genetic code (shCherbak, 2008). Both Freudenthal's LINCOS pattern and Dutil's and Dumas' improvement bear a striking likeness to the contents of this message.

First, the genetic code stores internally the fundamental symbols of arithmetic. They are: the zero, the decimal place-value number system, and numerous summations of nucleonsa kind of "ostensive numerals"-in amino acids. The decimalism shows itself through criterion of divisibility by the prime number 37 . There is a set of nucleon sums 000,111 , $222,333,444,555,666,777,888,999$ in the message. The decimal syntax of these sums is reinforced with their exact equilibrations. Another numerical symbol is the "Egyptian triangle". Such arithmetic asserts the artificial nature of the message and shows a possible mathematical order of genomes.

Second, the natural series and zero on its flank align the triplet bases. Such grammar discloses the so-called cooperative symmetry that is the message proper. A reader can recognize the symbols of DNA molecule, TATA-box, intron as well as genomic spacer as a possible final address of the message principal part. The spacer symbol is a palindrome written by the code symbols Start and Stop within the code itself. It is as if the genetic code had "known" before its own origin how to code for these syntactic signs (as well as all other coding) in order to do inside itself the palindrome. It could only be possible if the genetic code was projected preliminarily. By the way, the palindrome solves a problem of the privileged direction of reading. It simple does both these directions semantically identical. Third, stated above artificiality of the message may affect the origin of life. 
Cherbak V., (2008). The Arithmetical Origin of the Genetic Code. Barbieri M. (ed.), The Codes of Life: The Rules of Macroevolution. Springer (http://www.springerlink.com/ content/t85w0h771510j187/).

Dutil Y., Dumas S. (2003). Active SETI Page—http://www.active-seti.org/evpatoria_2003.jpg. Freudenthal, H. (1960). LINCOS: Design of a Language for Cosmic Intercourse. Amsterdam: North-Holland Publishing Company.

E-mail: genecodelab@hotmail.com 


\section{Origins of Homochirality}

\section{Chiroptical Properties of Amino and Diamino Acids: A Density Functional Theory Study}

Martine Adrian-Scotto, Uwe Meierhenrich

L.C.M.B.A (UMR 6001), Universit de Nice-Sophia Antipolis, Parc Valrose, 06108 NICE Cedex 2, France

Amino acids and diamino acids are involved in many scenarios elucidating possible origins of life on Earth. Amino acids were parts of early proteins (enzymes) and even their order of recruitment has been estimated (Jordan et al, 2005). Diamino acids might have served as molecular building blocks of an early genetic material such as peptide nucleic acid (PNA) (Nelson et al., 2000, Meierhenrich et al, 2004). One of the well-known challenges when discussing about biopolymers such as enzymes and oligonucleotides in living organisms is the phenomenon that these polymers implement monomers of exclusively one handedness, a phenomenon called homochirality. Fascinatingly, biopolymers are not composed of racemic monomers. Many attempts have been made in order to understand the process of racemic symmetry breaking (Borchers et al., 2004). Assuming an extraterrestrial origin of the molecular building blocks amino acids and diamino acids, their susceptibility to asymmetric photolysis in interstellar space was proposed, in connection with the absorption of circularly polarized electromagnetic radiation (Meierhenrich and Thiemann, 2004). To investigate electronic and chiroptical properties of amino and diamino acids more precisely, we called upon a quantum molecular modelling approach based on Density Functional Theory. We have studied here various molecules with the help of B3LYP computations using the basis functions $6-31 \mathrm{G}(\mathrm{d}, \mathrm{p})$. In particular, the circular dichroic behaviour of amino and diamino acids is discussed versus their computed corresponding spectra. The obtained results are compared with existing experimental data (Bredehft et al., 2007).

Borchers A.T., Davis P.A. and Gershwin E. (2004). The Asymmetry of Existence: Do We Owe Our Existence to Cold Dark Matter and the Weak Force?, Experimental Biology and Medecine, 229(1): 21-32.

Bredehft J. H., Breme K., Meierhenrich U. J., Hoffmann S.V. and Thiemann W. H.-P. (2007). Chiroptical Properties of Diamino Carboxylic Acids. Chirality, 19:570-573.

Jordan I.K., Kondrashov F.A., Adzhubei I.A., Wolf Y.I., Koonin E.V., Kondrashov A.S. and Sunyaev S. (2005). A universal trend of amino acid gain and loss in protein evolution, Nature, 433:633-638.

Meierhenrich U. J., Muoz Caro G.M., Bredehft J.H., Jessberger E.K. and Thiemann W. H.P. (2004). Identification of diamino acids in the Murchison meteorite, Proceedings of the National Academy of Sciences of the United States of America, 101(25):9182-9186.

Meierhenrich U. J. and Thiemann W. H.-P. (2004). Photochemical concepts on the origin of biomolecular asymmetry, Origins of Life and Evolution of the Biosphere, 34:111-121.

Nelson K. E., Levy M. and Miller S. L. (2000). Peptide nucleic acids rather than RNA may have been the first genetic molecule, Proceedings of the National Academy of Sciences of the United States of America, 97(8): 3868-3871.

E-mail: scotto@unice.fr 


\section{A Model for Asymmetric Amino Acid Photolysis}

Jan Hendrik Bredehöft ${ }^{1}$, Uwe J. Meierhenrich ${ }^{2}$, Katharina Breme ${ }^{2}$, Jun-ichi Takahashi ${ }^{3}$, Wolfram H.-P. Thiemann ${ }^{4}$, Søren V. Hoffmann ${ }^{5}$

${ }^{1}$ The Open University, Physics \& Astronomy, Walton Hall, Milton Keynes, MK7 6AA, United Kingdom; ${ }^{2}$ University of Nice-Sophia Antipolis, CNRS UMR 6001, avenue Valrose, 06108 Nice, France; ${ }^{3}$ NTT Microsystem Integration Laboratories, 3-1, Morinosato Wakamiya, Atsugi 243-0198, Japan; ${ }^{4}$ University of Bremen, Dept. of Physical Chemistry, Leobener Straße, 28359 Bremen, Germany; ${ }^{5}$ University of Aarhus, Institute for Storage Ring Facilities, Ny Munkegade, 8000 Aarhus C, Denmark

All biopolymers rely on a specific handedness of their building blocks, so the question of symmetry breaking occurs naturally when one tries to understand the origin and formation history of these biopolymers. It does so especially in proteins and their monomer building blocks, amino acids, since a very large number (90) of the latter are known to be found in extraterrestrial sources such as meteorites (Bredehöft and Meierhenrich in press). Some of these amino acids, clearly of non-biological origin, show an excess of one enantiomer over the other (Pizzarello and Cronin 2000). One of the mechanisms discussed for triggering this break of symmetry is asymmetric photochemistry in interstellar/ circumstellar matter by means of circularly polarized light (Bailey et al. 1998, Lucas et al. 2005, Buschermöhle et al. 2005, Meierhenrich et al. 2005). A very powerful tool for the study of the molecules that undergo such photochemical reactions is Circular Dichroism Spectroscopy. CD Spectroscopy can yield information on the susceptibility to asymmetric photochemistry of a substance, the wavelengths at which asymmetric photoreactions can occur and the direction of the chiral bias of such a reaction.

We recorded the CD spectra of several amino acids, among them proteinogenic as well as non-proteinogenic $\alpha-\mathrm{H}$ and $\alpha$-methyl amino acids and diamino acids in different liquid solvents (Bredehöft et al. 2007) and in the solid phase. Based on these spectra and quantum mechanical calculations, a model will be presented that illustrates the nature of the electronic excitation that is involved in the asymmetric photolysis of amino acids. This shows that indeed a single kind of photochemical reaction is sufficient to account for the asymmetric photolysis of most amino acids. Furthermore, the differences between spectra recorded under various conditions and the impact on asymmetric photochemistry that these conditions have will be discussed.

Bailey, J., Chrysostomou, A., Hough, J. H., Gledhill, T. M., McCall, A., Clark, S., Ménard, F., and Tamura, M., (1998). Circular Polarization in Star-Formation Regions: Implications for Biomolecular Homochirality, Science 281:672-674.

Bredehöft, J. H., Breme, K., Meierhenrich, U. J., Hoffmann, S. V., Thiemann, W. H.-P. (2007). Chiroptical Properties of Diamino Carboxylic Acids, Chirality, 19:570-573.

Bredehöft, J. H. and Meierhenrich, U. J. (in press), Amino acid structures from UV irradiation of simulated interstellar ices, in Takenaka, N. (Ed), Recent Developments in (Photo-)Chemistry in Ice, Research Signpost, Kerala, India.

Buschermöhle, M., Whittet, D. C. B., Chrysostomou, A., Hough, J. H., Lucas, P.W., Adamson, A. J., Whitney, B. A. and Wolff, M. J. (2005). An Extended Search for Circularly Polarized Infrared Radiation from the OMC-1 Region of Orion, Astrophys J, 624:821-826. 
Lucas, P.W., Hough, J. H., Bailey, J., Chrysostomou, A., Gledhill, T. M., McCall, A. (2005). UV Circular Polarisation in Star Formation Regions: The Origin of Homochirality?, Orig. Life Evol. Biosph. 35:29-60.

Meierhenrich, U. J., Nahon, L., Alcaraz, C., Bredehöft, J. H., Hoffmann, S. V., Barbier, B., Brack, A. (2005). Asymmetric vacuum UV photolysis of the amino acid leucine in the solid state, Angew. Chem. Int. Ed., 44:5630-5634.

Pizzarello, S., Cronin, J. R., (2000). Non-racemic amino acids in the Murray and Murchison meteorites, Geochem. Cosmochem. Acta, 64(2):329-338

E-mail: j.h.bredehoft@open.ac.uk

\section{A Possible Astrophysical Pathway to the Origin of Enantiomeric Excess in Primitive Meteorites: Laboratory Simulations}

P. de Marcellus ${ }^{1}$, G. Danger ${ }^{1}$, L. Nahon ${ }^{2}$, U. Meierhenrich ${ }^{3}$, L.d'Hendecourt ${ }^{1}$

${ }^{1}$ Astrochimie et Origines, Institut d'Astrophysique Spatiale, Orsay, France; ${ }^{2}$ Synchrotron SOLEIL, L'Orme des Merisiers-BP 48, 91190 Saint-Aubin, France; ${ }^{3}$ Laboratoire A.S.I. et Laboratoire de Chimie Bioorganique, UMR 6001 CNRS, Universite de Nice-Sophia Antipolis, Faculte des Sciences, Parc Valrose, F-06108 Nice Cedex 2, France

Evolution of organic matter in astrophysical environments, and in particular the formation of prebiotic molecules in the interstellar medium (ISM), is the aim of many researches. In this context, experimental simulations in laboratory have shown that a large quantity of amino acids can be formed by simple vacuum ultraviolet (VUV) irradiation of interstellar ice analogs. These abiotic syntheses of amino acids only lead, without asymmetric induction, to the formation of racemic mixtures (Bernstein et al. 2002; Muñoz-Caro et al. 2002). In meteorites such as Murchison or Murray, amino acids have been detected (Cronin et al. 1980). The origin of these meteoritic amino acids could be related to the photochemistry of ice analogs. Interestingly, some of these meteoritic amino acids do present enantiomeric excesses (e.e.) in their 1 form, which is the same configuration as amino acids included in biologic proteins (homochirality 1) (Cronin et al. 1999; Pizzarello et al. 2000; Pizzarello et al. 2003). Thereby, some authors have proposed a link between these meteoritics e.e. and the apparition of homochirality on Earth, through amplification processes (Reisse et al. 2003). One of the astrophysical hypotheses which could explain this meteoritic asymmetry is the irradiation of interstellar ices with UV circularly polarized light (UV-CPL) (Bailey, 2001). Using UV-CPL irradiation, experiments have shown that small e.e.s are formed from racemic substances by enantioselective photodegradation (Meierhenrich et al. 2005). To test this hypothesis in a more realistic scenario, our group investigates the possibility to obtain amino acids with e.e. by irradiating interstellar ice analogs with UV-CPL (Nuevo et al. 2007; Nuevo et al. 2006). The first results obtained with the SU5 beamline at LURE (Orsay, France) did not produce a clear evidence for this mechanism but obtained amount of materials were not sufficient for robust e.e. quantification. We will reproduce these experiments in September 2008 with the new UV beamline DESIRS of SOLEIL synchrotron which will allow for the formation of more organic matter and should improve the e.e.s sensitivity detection.

Bailey, J., (2001) Origins Life Evol. Biosphere, Astronomical sources of circularly polarized light and the origin of homochirality, 31:167-183. 
Bernstein, M. P., Dworkin, J. P., Sandford, S. A., Cooper, G. W., Allamandola, L. J., (2002) Racemic amino acids from the ultraviolet photolysis of interstellar ice analogues, Nature, 416:401-403.

Cronin, J. R., Candy, W. E., Pizzarello, S., Amino Acids of the Murchison Meteorite, 1980. Cronin, J. R., Pizzarello, S., Adv. Space Res. (1999) Amino acid enantiomeric excesses in meteorites: Origin and significance, 23:293-299.

Meierhenrich, U. J., Nahon, L., Alcaraz, C., Bredehft, J. H., Hoffmann, S. V., Barbier, B., Brack, A., (2005) Asymmetric Vacuum UV photolysis of the Amino Acid Leucine in the solid state, Angew. Chem., Int. Chem., 44:5630-5634.

Muñoz-Caro, G. M., Meierhenrich, U. J., Schutte, W. A., Barbier, B., Arcones Segovia, A., Rosenbauer, H., Thiemann, W. H.-P., Brack, A., Greenberg, J. M., (2002), Amino acids from ultraviolet irradiation of interstellar ice analogues, Nature, 416: 403-406.

Nuevo, M., Meierhenrich, U. J., Muoz-Caro, G. M., Dartois, E., d'Hendecourt, L., Deboffle, D., Auger, G., Blanot, D., Bredehoft, J. H., Nahon, L., (2006) The effects of circularly polarized light on amino acid enantiomers produced by the UV irradiation of interstellar ice analogs, Astron. Astrophys., 457:741-751.

Nuevo, M., Meierhenrich, U. J., d'Hendecourt, L., Muoz-Caro, G. M., Dartois, E., Deboffle, D., Thiemann, W. H.-P., Bredehoft, J.-H., Nahon, L., (2007) Enantiomeric separation of complex organic molecules produced from irradiation of interstellar/ circumstellar ice analogs, Adv. Space Res., 39, 400-404.

Pizzarello, S., Cronin, J. R., (2000) Geochem. Cosmo. Acta, Non-racemic amino acids in the Murray and Murchison meteorites, 64:329-338.

Pizzarello, S., Zolensky, M., Turk, K. A., (2003) Geochem. Cosmo. Acta, Nonracemic isovaline in the Murchison meteorite: Chiral distribution and mineral association, 67:1589-1595.

Reisse, J., Cronin, J., in: Bordeaux, P. U. d. (Ed.), Les traces du vivant, Presses Universitaires de Bordeaux, Bordeaux 2003, pp. 82-113.

E-mail: Louis.DHendecourt@ias.u-psud.fr

\title{
The Salt-Induced Peptide Formation Reaction as Possible Origin of Biohomochirality
}

\author{
Daniel Fitz, Bernd M. Rode
}

Division of Theoretical Chemistry; Institute of General, Inorganic and Theoretical Chemistry; University of Innsbruck

The Salt-Induced Peptide Formation (SIPF) Reaction has been shown to yield considerable amounts of di- and oligopeptides from amino acids in aqueous solution under assumed prebiotic conditions just with the help of sodium chloride and $\mathrm{Cu}(\mathrm{II})$ ions. Strikingly, a few amino acids, especially alanine (Plankensteiner, et al. 2004) and valine (Plankensteiner, et al. 2005), show better reactivity when present in their L-form compared to their Denantiomers, suggesting that this reaction might have played a keyrole in the origin of biohomochirality.

This behaviour may be explained by the geometry of the active, peptide-forming species. Under the reaction conditions a central copper ion forms a complex containing two amino acids and one choride ligand in a distorted square 'plane'. This distortion gives rise to central chirality at the copper ion, which, because of its relatively high atomic number, can now provide considerably high parity-violating energy differences (PVEDs, caused by parity violation in weak interactions) between a complex containing L-amino acids and its 
D-analogue. Ab initio geometry calculations of such active complexes show that the out-ofplane distortion of the ligands is more pronounced for amino acids showing an enantiomeric preference for the L-form than for those which do not (Fitz, et al. 2007). This means that central chirality at the copper center caused by out-of-plane distortion of the main ligands may give rise to higher PVEDs and, therefore, to markedly different chemical properties of L- and D-amino acid complexes.

Fitz, D., Reiner, H., Plankensteiner, K., and Rode, B. M. (2007). Possible origins of biohomochirality. Current Chemical Biology, 1:41-52.

Plankensteiner, K., Reiner, H., and Rode, B. M. (2005). Stereoselective differentiation in the Salt-induced Peptide Formation reaction and its relevance for the origin of life. Peptides, 26:535-541.

Plankensteiner, K., Righi, A., Rode, B. M., Gargallo, R., Jaumot, J., and Tauler, R. (2004). Indications towards a stereoselectivity of the salt-induced peptide formation reaction. Inorganice Chimica Acta, 357:649-656.

E-mail: Daniel.Fitz@uibk.ac.at

\section{Chiral Crystals of Achiral Biological Compounds as an Origin of Homochirality of Biomolecules in Conjunction with Asymmetric Autocatalysis}

Tsuneomi Kawasaki ${ }^{1}$, Kenta Suzuki ${ }^{1}$, Yuko Hakoda ${ }^{1}$, Kunihiko Hatase ${ }^{1}$, Yuuki Harada ${ }^{1}$, Nicola Florini ${ }^{2}$, Gyula Pályi ${ }^{2}$, Kenso Soai ${ }^{1 *}$

${ }^{1}$ Department of Applied Chemistry, Tokyo University of Science, Kagurazaka, Shinjuku-ku, Tokyo 162-8601, Japan; ${ }^{2}$ Department of Chemistry, University of Modena and Reggio Emilia, via G Campi, 183-41100 Modena, Italy

The homochirality of biomolecules such as L-amino acids and D-sugars is one of the essential features of life and has been a puzzle for the chemical origin of life. It is known that some achiral organic compounds crystallize in chiral forms and which has been an important candidate for the origin of chirality.

Considering the significant enantioenrichments in biological system, chirality of these crystals should be transferred to other organic compounds with amplification of the quantity and enantioenrichment in the prebiotic world. We previously reported the asymmetric reaction mediated by chiral organic crystal as chiral initiators. The chiral crystals serve as chiral initiators of asymmetric autocatalysis (Soai and Kawasaki 2006) and the quantity of chirality has been significantly amplified to achieve the large amount of highly enantioenriched compound (Kawasaki, et al. 2005).

In this presentation, we show that cytosine, a prebiotic achiral biomolecule and a nucleobase, spontaneously forms enantioenriched crystals by stirred crystallizations, and the crystal of cytosine acts as a chiral initiator for asymmetric autocatalysis, providing a near enantiopure pyrimidyl alkanol (Kawasaki, et al. 2008). The enantiomorphous onecomponent single crystals of hippuric acid ( $N$-benzoylglycine), which is an achiral naturally occurring amino acid derivative, also acts as the source of chirality in asymmetric autocatalysis (Kawasaki, et al. 2006). To expand the utility of chiral crystal formed from achiral organic compound for the origin of chirality in asymmetric autocatalysis, we subjected the chiral crystals of benzil and its derivative to the autocatalytic reaction. These results are also discussed. 


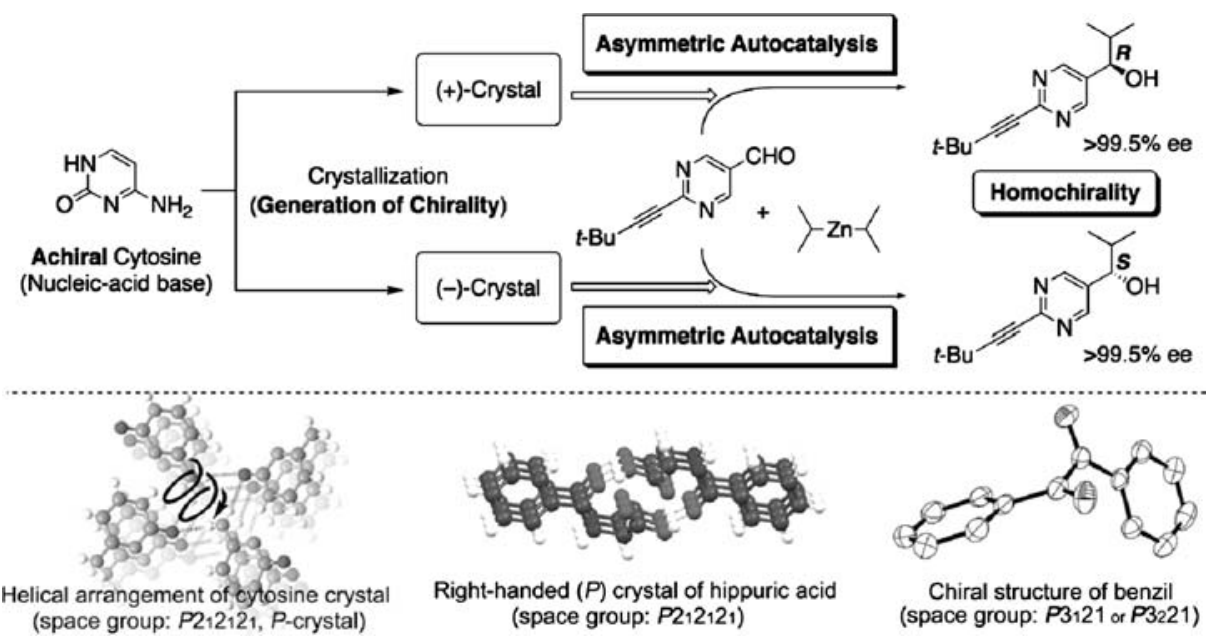

Kawasaki, T., Jo, K., Igarashi, H., Sato, I., Nagano, M., Koshima, H., and Soai, K. (2005). Asymmetric amplification using chiral co-crystal formed from achiral organic molecules by asymmetric autocatalysis. Angew. Chem. Int. Ed., 44:2774-2777.

Kawasaki, T., Suzuki, K., Hakoda, Y., and Soai, K. (2008). Achiral nucleobase cytosine acts as an origin of homochirality of biomolecules in conjunction with asymmetric autocatalysis. Angew. Chem. Int. Ed., 47:496-499.

Kawasaki, T., Suzuki, K., Hatase, K., Otsuka, M., Kashima, H., and Soai, K. (2006). Enantioselective synthesis mediated by chiral crystal of achiral hippuric acid in conjunction with asymmetric autocatalysis. Chem. Commun., 1869-1871.

Soai, K., and Kawasaki, T. (2006). Discovery of asymmetric autocatalysis with amplification of chirality and its implications in chiral homogeneity of biomolecules. Chirality, 18:469-478.

E-mail: soai@rs.kagu.tus.ac.jp

\section{Studies on Chirality: Enantioselectivity in Ion-Molecule Gas Phase Reactions}

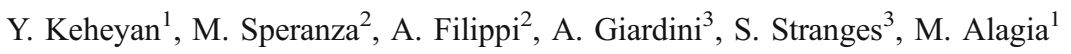

${ }^{1}$ ISMN-CNR, c/o Dept. of Chemistry, University "La Sapienza", p.le Aldo Moro 5, Rome0185, Italy; ${ }^{2}$ Dipt. Degli Studi di Chimica e tecnologia delle Sostanze Biologicamente Attive, Università "La Sapienza", 00185 Roma, Italy; ${ }^{3}$ Dipt. di Chimica, Università "La Sapienza" 00185 Roma, Italy

Virtually all biological processes involve chiral molecules of appropriate shape and size maintaining suitable functionalities in specific positions. Their specific interactions with appropriate receptors is at the basis of chiral recognition and biocatalysis. The very complex molecules that make up living organisms, such as DNA, RNA, proteins and sugars, are all chiral. One of the most remarkable facts in biology is that the biomolecular chirality, be it in virus, in a primitive bacterium, or in a human brain cell, is everywhere the same. In recent years, considerable progress has been made in the study of weakly bonded molecular complexes between chiral molecules in the gas phase using laser spectroscopy combined with supersonic beam. 
The results of these studies are particularly useful since they refer to isolated systems unperturbed by environmental effects and, therefore, directly comparable to theoretical predictions. Resonant Two Photon Ionization (R2PI) Spectroscopy, coupled with time of flight (TOF) mass spectrometry, on cooled complexes in supersonic beam is an excellent tool for investigating the structure and the specific intermolecular interactions in hydrogenbonded clusters between chiral aromatic alcohols and a variety of solvent molecules, including chiral mono- and bi-functional alcohols, amines and water. Recently this methodology to the study of $R$-1-phenyl-2,2,2-trifluoroethanol has been applied. The interaction of polarized light with chiral systems has been studied. The circularly polarized light of POLAR beamline at ELETTRA synchrotron experiments will be reported for some chiral molecules.

E-mail: yeghis.keheyan@uniroma1.it

\section{Interconversion and Degradation of Dipeptide Diastereomers Induced by $\gamma$-Rays Irradiation}

Toratane Munegumi ${ }^{1}$, Akihiko Shimada ${ }^{2}$, Kenji Asahi ${ }^{1}$, Michio Takada ${ }^{1}$, Tsuyoshi Fujimoto $^{1}$, Nozomi Nagashima ${ }^{1}$, Akira Shimoyama ${ }^{3}$

\section{${ }^{1}$ Oyama National College of Technology; ${ }^{2,3}$ Kochi Gakuen College}

Origin and development of homochirality of living organisms have been discussed for many years (Bonner, 1991; Akaboshi, et al. 2000; Plasson, et al. 2007). There seem to be three questions related to this discussion. The first is "L or D?" The second is "Homochiral or Heterochiral?" The third is "different or same?" The first two points concern with homochirality of each biopolymer like protein and nucleic acid. The last questions concerned with the combination of homochirality between different biopolymers.

This research involves answering the second question: "Homochiral or heterochiral?" We already reported a hypothesis on the question: "Homochiral or heterochiral?" related to peptides and proteins (Munegumi and Shimoyama, 2003). The report insisted the importance of the difference in hydrophobicity between homochiral and heterochiral oligopeptides in the development of homochirality. And a scenario for the development of homochirality of peptides was proposed (Munegumi and Shimoyama, 2003). The scenario includes separation of diastereomeric peptides and stereo-selective reactions (Munegumi, et al. 2005). The scenario also focused on several energy sources (Munegumi, et al. 2005) which might have induced such stereo-selective reactions.

This research includes epimerization and degradation of oligopeptides induced by $\gamma$-rays irradiation, which seems to be an important energy source to produce organic compounds (Akaboshi, 2000).

Linear or cyclic dipeptides (L-Ala-L-Ala, D-Ala-L-Ala, L-Ala-Gly, Gly-L-Ala and

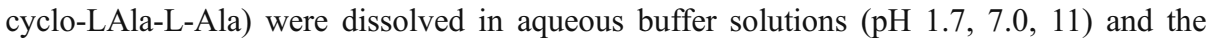
resulted $1 \mathrm{mM}$ solutions were irradiated by $\gamma$-rays $(2,4,8,16,24 \mathrm{kGy})$. The reaction solutions were analyzed by means of an amino acid analyzer and a reversed phase HPLC system. Homochiral peptide L-Ala-L-Ala yielded its diastereomers (heterochiral peptides: L-Ala-D-Ala and D-Ala-L-Ala; total yield: c.a. $6 \%$ at $\mathrm{pH} 1.7,4 \mathrm{kGy}$ ) with the $\gamma$-rays irradiation. Degradation of peptide L-Ala-L-Ala to Ala, Ala- $\mathrm{NH}_{2}$ (alaninamide) and ammonia was observed under the every reaction conditions. All the peptide substrates almost degraded (recovery <1\%) under smaller dose than 16 kGy except of $\mathrm{pH} 11$. 
Dipeptide cyclo-L-Ala-L-Ala rapidly degraded rather than linear dipeptides. However, inerconversion of cyclo-L-Ala-L-Ala to its diastereomer was observed only at $\mathrm{pH} 11$. Although Interconversion of homochiral to heterochiral peptides was faster than that of heterochiral to homochiral peptides at $\mathrm{pH}$ 1.7, interconversion of heterochiral to homochiral peptides was faster than the other at $\mathrm{pH} 11$. These results afford important information to discuss the conditions ( $\mathrm{pH}$ and irradiation) and mechanisms of development of homochirality of peptides and proteins.

Akaboshi, M., Fuji, N., and Navarro-Gonzalez, R. editors (2000). The role of radiation in the origin and evolution of life. Kyoto University Press, Kyoto.

Bonner, W. A. (1991). The Origin and amplification of biomolecular chirality. Origins of Life and Evolution of Biosphere, 21:59-111.

Munegumi, T. and Shimoyama, A (2003). Development of homochiral peptides in the chemical evolutionary process: separation of homochiral and heterochiral oligopeptides. Chirality,15: S108-S115.

Munegumi, T., Takayama, N., Ebina, T. and Sawahata, M. (2005). Stereo-specific condensation of activated amino acids or peptides. Viva Origino, 33:151-151.

Plasson, R., Kondepudi, D. K., Bersini, H., Commerras, A., and Asakura, K. (2007). Emergence of homochirality in far-from-equilibrium systems: mechanisms and role in prebiotic chemistry. Chirality, 19: 589-600.

E-mail: munegumi@oyama-ct.ac.jp

\section{Small Structural Change Producing Tryptophanase Activity on D-tryptophan}

Akihiko Shimada

Sustainable Environmental Studies, Graduate School of Life and Environmental Sciences, University of Tsukuba, Tsukuba, Japan

Tryptophanase (TPase) is an enzyme with extremely tight stereospecificity, cleaving 1 tryptophan into indole, having no activity on D-tryptophan under ordinary conditions. However, it becomes active toward d-tryptophan in highly concentrated ammonium phosphate solutions quite different from what was expected. The only salts inducing the reaction were diammonium phosphate, triammonium phosphate and ammonium sulfate, although other salts didn't have the activity at all. Free tryptophan is more readily influenced by alkaline $\mathrm{pH}$ or strong ion strengths than other biological amino acids. If ammonium phosphates affect chemical racemization on D-tryptophan, the enzymological significance of this reaction is lost. So it is important to demonstrate that ammonium phosphates do not racemize free D-tryptophan at all. We used an HPLC column appropriate for tryptophan resolution to analyze free D-tryptophan, demonstrating that the reaction is enzymatic metabolism (Shimada, 2007). Ammonium phosphates as diammonium hydrogenphosphate or triammonium phosphate probably produce structural change in tryptophanase, which makes it possible that activity on Dtryptophan will emerge. This result indicates enzyme stereospecificity is more flexible than we think. Judging from the flexibility of tryptophanase stereospecificity, this conformational change is maybe small. Circular dichroism analyses were thus applied to tryptophanase in ammonium phosphate solution. A $200 \mu \mathrm{L}$ of monoammonium hydrogenphosphate (MAP), diammonium hydrogenphosphate (DAP), and triammonium 
phosphate (TAP) of 50\% saturation and phosphate buffer (PB) solutions with $0.5 \mu \mathrm{M}$ of apoTPase and $1.1 \mathrm{mM}$ of PLP was injected in a $0.1 \mathrm{~cm}$ path length cell in a circular dichroism (CD) spectrophotometer. Spectra were recorded at wavelengths from 200 to $350 \mathrm{~nm}$ at room temperature. Five scans were repeated per a spectrum, averaged, and expressed as molar ellipticity in degrees $\mathrm{cm}^{2} \mathrm{dmol}-1$. The CD spectra were calculated by subtracting a blank prepared identically but without TPase, and also analyzed with a CDPro software package. CD spectra in the near-uv region $(250-350 \mathrm{~nm})$ did not produce any difference among PB, TAP, DAP, and MAP, indicating that TPase had normal tertiary structure in highly concentrated ammonium phosphate solutions. On the other hand, CD spectra in the far-uv region $(200-250 \mathrm{~nm})$ produced subtle but detectable differences, indicating that ammonium phosphates produced changes in the secondary structure of TPase. Theses spectra are useful for assessing the degree to which ammonium phosphates change it. Choosing $\lambda=220 \mathrm{~nm}$ as the single wavelength for monitoring specific features of the protein structure, we compared the signal at this wavelength among TAP, DAP, and MAP. When the degree of conformational change was defined as $100 \%$ unfolding in the MAP solution, it was $10 \%$ in DAP and $7 \%$ in TAP. Measurement of the CD spectra showed that a limited secondary structural change was required for TPase activity to appear on D-Trp. Judging from fluorescence and CD measurements, the degree of conformational change is very small. D-tryptophan is inactive in the absence of ammonium phosphates, so it might be concluded that it does not interact with Dtryptophan. However, kinetic studies show competitive interaction between active site of tryptophanase and D-tryptophan. We can tell that D-tryptophan binds to tryptophanase without ammonium phosphates. This fact seems to offer hint of a solution of the question that D-amino acids are unilaterally excluded. It therefore becomes important to identify a binding form of D-tryptophan at the active site of tryptophanse. It is inferred based on spectrophotometric analysis in the future researches, offering insights into how tryptophanase excludes only the D form.

Shimada, A. (2007). Role of ammonium phosphates in tryptophanase activity toward Dtryptophan. In Konno, R. et al., editors, D-amino acids: A New Frontier in Amino Acid and Protein Research-Practical Methods and Protocols, pages 591-607. Nova Science Publishers, New York.

E-mail: ashimada@kankyo.envr.tsukuba.ac.jp

\section{Asymmetric Synthesis and Decomposition of Amino Acids by Circularly Polarized Light from Free Electron Laser}

Tomoya Ogawa ${ }^{1}$, Soichiro Shima ${ }^{1}$, Takeo Kaneko ${ }^{1}$, Kensei Kobayashi ${ }^{1}$,Jun-ichi Takahashi ${ }^{2}$, Hajime Mita ${ }^{3}$, Masato Hosaka ${ }^{4}$, Masahiro Kato ${ }^{5}$

${ }^{1}$ Graduate School of Engineering, Yokohama National University, Yokohama 240-8501, Japan; ${ }^{2}$ NTT Microsystem Integration Laboratories, Atsugi 243-0198, Japan; ${ }^{3}$ Faculty of Engineering, Fukuoka Institute of Technology, Fukuoka 811-0295, Japan; ${ }^{4}$ Graduate School of Engineering, Nagoya University, Nagoya 464-8601, Japan; ${ }^{5}$ UVSOR, Institute for Molecular Science, Okazaki 444-8585, Japan

The origin of homochirality of biological molecules such as amino acids has remained one of the most important problems in the field of origins of life and astrobiology. 
Cronin and Pizzarello reported that some amino acids extracted from carbonaceous chondrites showed significant enantiomeric excesses of L-isomers (Cronin and Pizzarello, 1997). Isovaline, a non-proteinous amino acid without a-hydrogen atom, was included in such category of amino acids. One of the possible scenario for the generation of enantiomeric excesses of amino acids are asymmetric formation or decomposition of amino acids by circular polarized light in space. Bailey found circular polarized light of IR range in space (Bailey, et al. 1998). Takano et al. reported that enantiomeric excess of alanine was formed after irradiation of amino acid precursors with UV-CPL (Takano, et al. 2007). Here we examine decomposition of isovaline by irradiation with UV-CPL from UVSOR-free electron laser (FEL). We also studied possible introduction of chirality to amino acids in thin films by UV-CPL irradiation. Aqueous solution of isovaline in a quartz cell was irradiated with UV-CPL. After either R- or L-UV-CPL (wavelength: 216-230 nm) was irradiated, amino acids and amines in resulting products were analyzed by cation-exchange HPLC (Shimadzu LC-10A), and carboxylic acids were determined by capillary electrophoresis (Photal CAPI-3300). D/L ratio of amino acids was measured by reversed-phase HPLC after AQC derivatization (Tosoh DP-8020). Isovaline aqueous solution was also irradiated with high-energy heavy ions (290 MeV/u carbon ions from HIMAC, NIRS, Japan) or $\mathrm{X}$-rays $(6 \mathrm{keV}, 27 \mathrm{~B}$ line of Photon Factory, KEK, Japan). Thin film of phenylalanine was made by vacuum deposition on an $\mathrm{MgF}_{2}$ substrate. The film was irradiated with $\mathrm{D}$ or L-CPL. CD spectra were measured after irradiation. A gaseous mixture of carbon monoxide, ammonia and water was also irradiated with UV-CPL to examine possible formation of amino acid precursors. The resulting product was acid-hydrolyzed, and amino acids were determined by HPLC (Shimadzu LC-10A). When isovaline solution was irradiated with UV-CPL, isovaline was decomposed: Alanine was found as predominant amino acid products, and 2-butylamine and isovaleric acid were also detected. The release of methyl group, carboxylic group, or amino group from isovaline was specific to UV irradiation, since X-rays or heavy ions irradiation of isovaline solution did not give them as major products. Enantiomeric excesses of isovaline or alanine were not detected in the present experiments. As $\mathrm{pH}$ of the solution might be important for asymmetric decomposition, we plan to irradiate isovaline solution in acidic/basic conditions. When phenylalanine thin films were irradiated L- or R-CPL, the resulting films showed apparent CD spectra at $200 \mathrm{~nm}$ and $220 \mathrm{~nm}$. They seem to correspond to $\pi-\pi^{*}$ and $\mathrm{n}-\pi^{*}$ transitions, individually. It was proved that CPL irradiation introduced chirality to thin film of aromatic amino acids. Amino acids were formed by UV-CPL irradiation of the gas mixture: Glycine was predominant, followed by alanine. G-value of glycine was 0.0012 , which was smaller than that by proton irradiation or that with UV light from $\mathrm{D}_{2}$ lamp.

Bailey, J. A., Chrysostomou, A., Hough, J. H., Gledhill, T. M., McCall, A., Clark, S., Ménard, F., and Tamura, M. (1998). Circular polarization in star-formation regions: Implications for biomolecular homochirality. Science, 281: 672-674.

Cronin, J. R. and Pizzarello, S. (1997). Enantiomeric excesses in meteoritic amino acids. Science, 275: 951-955.

Takano, Y., Takahashi, J., Kaneko, T., Marumo, S., and Kobayashi, K. (2007). Asymmetric synthesis of amino acid precursors in interstellar complex organics by circularly polarized light. Earth and Planetary Science Letters, 254: 106-114.

E-mail: jitaka@ba2.so-net.ne.jp 


\section{Asymmetric Reactions of Amino-Acid-Related Compounds by Polarized Electrons from Beta-decay Radiation}

V. I. Burkov¹, L. A. Goncharova ${ }^{2}$, G. A. Gusev ${ }^{2}$, H. Hashimoto ${ }^{3}$, F. Kaneko ${ }^{4}$, T. Kaneko ${ }^{5}$, K. Kobayashi ${ }^{5}$, H. Mita ${ }^{6}$, E. V. Moiseenko ${ }^{7}$, T. Ogawa ${ }^{5}$, N. G. Poluhina ${ }^{2}$, T. Saito ${ }^{8}$, S. Shima ${ }^{5}$, J. Takahashi ${ }^{9}$, M. Tanaka ${ }^{4}$, Y. Tao ${ }^{10}$, V. A.Tsarev ${ }^{2}$, J. Xu$^{10}$, H. Yabuta ${ }^{11}$, K. Yagi-Watanabe $^{4}$, H. Yan ${ }^{10}$, G. Zhang ${ }^{12}$

${ }^{1}$ Moscow Institute of Physics and Technology, Institutsky per. 9, Dolgoprudnii, Moscow obl., 141700, Russia; ${ }^{2}$ P.N. Lebedev Physical Institute of the RAS, Leninsky Prospect 53, Moscow 119991, Russia; ${ }^{3}$ Department of Space and Astronautical Science, ISAS/JAXA, Sagamihara 229-8510, Japan; ${ }^{4}$ National Institute of Advanced Industrial Science and Technology, Tsukuba 305-8568, Japan; ${ }^{5}$ Graduate School of Engineering, Yokohama National University, Yokohama 240-8501, Japan; ${ }^{6}$ Faculty of Engineering, Fukuoka Institute of Technology, Fukuoka 811-0295, Japan; ${ }^{7}$ Russian Federal Nuclear Center, Snezhinsk, Chelyabinskaya obl., P.O. Box 589, Russia; ${ }^{8}$ Institute of Applied Science, Tokyo 160-0022, Japan; ${ }^{9}$ Science and Core Technology Laboratory Group, NTT, Atsugi 243-0198, Japan; ${ }^{10}$ Institute of High-Energy Physics, P.O. Box 918, Yuquanlu, District Beijing 100039, China; ${ }^{11}$ Department of Earth and Space Science, Osaka University, Toyonaka 560-0043, Japan; ${ }^{12}$ University of Science and Technology of China, NSRL, P.O. Box 6022, Hefei, Anhui 230029, China

The origin of homochirality of biological molecules such as amino acids has remained one of the most important problems in the field of origins of life and astrobiology. One of the possible scenario for the generation of enantiomeric excesses of amino acids are asymmetric formation or decomposition of amino acids by circularly polarized light from synchrotron radiation source in space (i.e. Takano, et al. 2007). However, one of the serious drawbacks of the hypothesis is that direction of circular polarization depends on relative position to the radiation source. Another possible hypothesis is based on the radiation source with absolutely determined polarization direction. It is well known that electrons from betadecay radiation advance with determined helicity derived from parity violence mechanism.

Tsarev et al. have reported the first results of the RAdiation Mechanism of Biomolecular ASymmetry (RAMBAS) experiment on investigation of the radiation mechanism of the influence on chiral molecules, as a factor leading to origination of chiral asymmetry (Burkoy, et al. 2008). It was found that irradiation of simple achiral materials by a flux of electrons from radioactive source initiated the synthesis of amino acids, and it resulted in asymmetric degradation and chiral asymmetry in a racemic mixture of amino acids. The results obtained can be important for the solution of the origin-of-life and biological homochirality problems.

We are planning further experiments on asymmetric reactions of amino-acid-related materials, such as amino-acid metal-complexes in solution or thin solid films on glass substrate surface, combined with circular dichroism (CD) measurements in vacuum ultraviolet (VUV) region using synchrotron radiation beam lines at Beijing and Tsukuba.

Burkov, V. I., Goncharova, L. A., Gusev, G. A., Kobayashi, K., Moiseenko, E. V., Poluhina, N. G., Saito, T., Tsarev, V. A., Xu Jianhua, and Zhang Guobin (2008). First Results of the RAMBAS Experiment on Investigation of the Radiation Mechanism of Chiral Influence. Origins of Life and Evolution of Biospheres 38:155-163. 
Takano, Y., Takahashi, J., Kaneko, T., Marumo, S., and Kobayashi, K. (2007). Asymmetric synthesis of amino acid precursors in interstellar complex organics by circularly polarized light. Earth and Planetary Science Letters, 254: 106-114. 


\title{
RNA World
}

\section{The Further Development of RNA Synthesis with Mineral Catalysis}

\author{
Michael F Aldersley, James P Ferris
}

Rensselaer Polytechnic Institute, Troy NY 12180 USA

Our studies have focused on the premise that minerals and metal ions catalyzed the formation of biopolymers that instituted the first Life on Earth.

Certain montmorillonites catalyze the formation of RNA oligomers that contain up to 50 monomer units determined by MALDI mass spectrometry and gel electrophoresis (Huang and Ferris, 2006; Zagorevskii et al., 2006).

In our system, montmorillonite is a catalyst that favours sequence selectivity and phosphodiester bond selectivity (Huang and Ferris, 2006; Miyakawa and Ferris, 2006). The present research takes this project is an entirely new direction using affinity chromatography. Initial studies established that our oligoribonucleotide products contain aptamers (RNA sequences that bind target molecules like amino-acid, nucleotides, co-enzymes, etc). We have demonstrated that the RNA oligomers can be separated by use of two affinity columns using different eluents (Cuatrecasas et al., 1977; Yasuda et al., 1983). A broad array of products is tested by merely changing the proportions of the initial activated monomers. Structural information on the oligomers that bind to the target will be obtained by mass spectrometry and by HPLC using a radiation detector. Representative results will be illustrated.

Cuatrecasas, P et al., Methods in Enzymology, 1977, 34, 77-102.

Huang, W,; Ferris, J.P., J. Am. Chem. Soc. 2006, 128, 8914-8919.

Miyakawa, S; Ferris, J.P., J. Am. Chem. Soc. 2006, 125, 8202-8208.

Yasuda, N.; Iwagami, H.; Nakanishi, E.; Nakamiya, T.; Sasaki, Y.; Murata, T., Journal of Antibiotics 1983, 36(3), 242-249.

Zagorevskii, D.V.; Aldersley, M.F.; Ferris J.P., J. American Society of Mass Spectrometry 2006, 17, 1265-1270.

E-mail: alderm@rpi.edu

\section{Creating de novo Random RNAs. Implication for the RNA-World}

Anella Fabrizio Maria ${ }^{1,2}$, De Lucrezia Davide ${ }^{1,2}$, Faiella Rachel $^{2}$, Chiarabelli Cristiano ${ }^{1,2}$, Luisi Pier Luigi ${ }^{1}$

${ }^{1}$ Department of Biology, University of RomaTre, 00146 Rome, Italy; ${ }^{2}$ European Centre for Living Technology, 30124Venice, Italy

The RNA World hypothesis, which assumes that an RNA World preceded our contemporary DNA/RNA/protein World, has become more and more popular in the field of the origin of life (Joyce, 2002; Orgel, 2003). Despite the recent progresses made in this field, some basic questions remain unanswered: Can RNA catalyse the reaction needed for self-replication on the early Earth? Can RNA-based life achieve the metabolic sophistication needed to give birth to the protein-nucleic acid World? To tackle to these questions a number of theoretical and experimental (Szostak et al., 2001; Muller, 2006) works have been carried out with the ultimate goal of re-creating an RNA World in the laboratory. Within this framework lies the 
"Never Born Biopolymers (NBBs)" project (Luisi et al., 2006) and in particular the "Never Born RNAs" (NBRs) project which goal is to explore the RNAs' sequence space for catalytic functions. This project moves from the observation that the extant collection of RNA molecules is only a minor part of the all theoretically possible RNA sequences (Luisi, 2003). On the basis of these observations, the question whether functionality is a common feature or a rare result of natural selection is of the utmost importance to elucidate the role of RNA in the origin of life and to fully exploit its biological potential.

In this work we report the investigation of the catalytic properties of a completely de novo library of random RNAs with the aim to determine whether and to what extent functional RNA spontaneously occur in a random library. A random DNA library of 60 residues was designed and produced to carry out in vitro transcription and the resulting RNAs was screened to evaluate their functional properties by means of in vitro evolution (Joyce, 2004). The population of RNAs was screened for the ability of recognized a Transition State Analogue (TSA) for the protease reaction (Yamauchi et al., 2002). According to the transition state theory (Eyring, 1935; Tanaka, 2002) enzymes catalyze chemical reactions by lowering the activation energy by recognizing and binding to the transient transition state structure as it is formed during the reaction. Based on this concept, TSA are designed to closely mimic the transition states and related high-energy intermediates with regards to bond orders, lengths, and angles, as well as expanded valences, charge distribution, and geometry. Accordingly, RNA sequences that bind to TSA molecules may be capable to catalyze the corresponding reaction. The results will be used to provide directions and suggestions for further studies concerning the functional properties of RNAs in the early evolution scenario.

Eyring, H. (1935): The activated complex in chemical reactions. J. Chem. Phys., 3: 107 Joyce, G.F. (2002): The antiquity of RNA-based evolution. Nature, 418: 214-221

Joyce, G.F. (2004): Directed Evolution of Nucleic Acid Enzymes. Annu Rev Biochem., 73: 791-836

Luisi, P.L. (2003): Contingency and determinism. Phil. Trans. R. Soc. Lond. A, 361: 1141-1147

Luisi, P.L., Chiarabelli, C. and Stano, P. (2006): From Never Born Proteins to Minimal Living Cells: Two Projects in Synthetic Biology. Orig. Life Evol. Biosph., 36: 605-616

Muller, U.F. (2006): Re-creating an RNA world. Cell. Mol. Life Sci., 63: 1278-1293

Orgel, L.E. (2003): Some consequences of the RNA world hypothesis. Orig. Life Evol. Biosph., 33: 211-218

Szostak, J.W., Bartel, D.P. and Luisi, P.L. (2001): Synthesizing life. Nature, 409: 387-390 Tanaka, F. (2002): Catalytic Antibodies as Designer Proteases and Esterases. Chem. Rev., 102: 4885-4906

Yamanuchi, A., Nakashima, T., Tokuriki, N., Hosokawa, M., Nogami, H., Arioka, S., Urabe, I. and Yomo, T. (2002): Evolvability of random polypeptides through functional selection within a small library. Protein Engineering, 15(7): 619-626

E-mail: fabibbo@libero.it

\section{Did DNA Come Before Proteins?}

Aaron S. Burton, Niles Lehman

Portland State University P.O. Box 751 Portland, Oregon 97207

The RNA World hypothesis describes a time when RNA molecules took on both catalytic and informational roles, prior to the advent of either DNA or proteins. There has been much 
debate as to which of those two came next. Transitioning from RNA to DNA as the hereditary molecule greatly improved genomic stability, increasing the likelihood that a given organism (or molecule) would be around long enough to reproduce. Turning over the role of primary catalyst to proteins offered significant advantages as well — a wider array of chemical reactions could be catalyzed at a much faster rate, again contributing to a heightened probability that an organism survives to reproduce. Either transition affords obvious benefits to a ribo-organism, but in fundamentally different ways, and would come about through very different evolutionary pathways. Arguments have been made for both sides. Simplicity favors DNA next (Benner et al., 1989; Dworkin et al., 2003), as fewer genes would be required to evolve DNA than protein synthesis. On the other hand, a compelling argument for proteins preceding DNA was made based on the difficulty of ribonucleotide reduction and homology of protein ribonucleotide reductases (Freeland et al., 1999). Here, based on recently discovered nucleic acid catalysts, we propose two possible routes by which RNA could have made DNA without the aid of any protein catalysts.

Benner, S.A., Ellington, A.D., and Tauer, A. (1989) Modern metabolism as a palimpsest of the RNA world. Proc. Natl. Acad. Sci. USA 86, 7054-7058.

Burton, A.S. and Lehman, N. (In review). DNA before proteins? Recent discoveries in nucleic acid catalysis strengthen the case. Astrobiology.

Dworkin, J.P., Lazcano, A. and Miller, S.L. (2003) The roads to and from the RNA world. J. Theor. Biol. 222, 127-134.

Freeland, S.J., Knight, R.D., and Landweber, L.F. (1999) Do proteins predate DNA? Science 286, 690-692.

Heine, A., DeSantis, G., Luz, J.G., Mitchell, M., Wong, C.H., and Wilson, I.A. (2001) Observation of covalent intermediates in an enzyme mechanism at atomic resolution. Science 294, 369-374.

E-mail: burtona2001@msn.com

\section{The Origin of Life as Seen Through a Regularity}

\section{Sacha Haywood ${ }^{1}$, Raphaëlle D. Haywood ${ }^{2}$}

${ }^{1} 12$ Avenue Victor Hugo, 89200 Avallon, France; ${ }^{2}$ Imperial College London, South Kensington Campus, London SW7 2AZ, UK

Charles Darwin (1859) and Alfred Russel Wallace (1870) are universally known for their demonstration of the importance of a lawlike principle or regularity - natural selection-in the origin of species. They are much less well known for their lifelong hostility towards the discovery of other genuine regularities that might be involved in the origin of species. Yet, all through the nineteenth and twentieth centuries, several lesser-known naturalists, most notably St. George Jackson Mivart (1871), have forcefully advocated the existence of other such regularities.

In a recent book, Haywood (2007) has argued that, in the process of evolutionary change, not one but two lawlike principles, or rather universal laws, can be recognized: natural selection and developmental determination. Broadly speaking, while the first law deals with the fate of inherited variation; the other, originally derived from the embryological concepts of competence, induction and determination, deals with its emergence. Universal laws assigned to the way evolution proceeds form the basis for a general lawlike understanding of the wider patterns of evolution. That of course includes 
the rise of complexity in the universe, which can indeed be associated with another regularity. It has been dubbed the law of major transitions.

By virtue of its simple logic, the law of major transitions allows the strict recognition of nineteen major evolutionary transitions to greater complexity. Eight of these coincide with what is commonly described as the origin of life. They span from the evolution of organic molecules, in particular amino acids (Major Transition or MT 7), to taxis-enabled prokaryotes (MT 14) via proteinoids (MT 8), catalytic proteinoids (MT 9), nucleic acids (MT 10), catalytic nucleic acids ( $M T$ 11), proteins (MT 12), and enzymes (MT 13). According to this evolutionary sequence drawn from a universal regularity, transcription evolved first, at the eleventh major transition, and translation second, at the twelfth major transition. Another prediction associated with the law of major transitions is that, before our enzyme world, there were a proteinoid world, followed by a DNA-RNA world and then a protein world, but no RNA world (Haywood, 2007).

Darwin, C. R. (1859). On the Origin of Species. John Murray, London.

Mivart, St. G. J. (1871). On the Genesis of Species. Macmillan \& Co., London.

Haywood, S. (2007). The Laws of Evolution and Derived Lawlike Principles. Hagenia, Oxford.

Wallace, A. R. (1870). Contributions to the Theory of Natural Selection. Macmillan \& Co., London.

E-mail: sacha.haywood@wolfson.oxon.org

\title{
Evolution of the Genetic Code in Terms of Conserved Proteins
}

\author{
Luz Caldern, Tzipe Govezensky, Marco V. Jos
}

Theoretical Biology Group, Instituto de Investigaciones Biomdicas, Universidad Nacional Autnoma de Mexico, Mexico D.F. 04510, Mexico

$\mathrm{RNY}$ repeating sequences, where $\mathrm{R}$ means purines, $\mathrm{Y}$ pyrimidine and $\mathrm{N}$ any of them, are considered to be relics of a primeval genetic code of the so-called RNA World. We proposed two plausible evolutionary paths, leading to two intermediate genetic codes, called Extended RNA Codes Type I and II, from which the primeval genetic code of RNA could have evolved to the Standard Genetic Code (SGC). Both, Extended RNA Code Type I and II are obtained by adding codons to the RNY sequences; to get the former code the codons resulting from frame reading mistranslations in the second and third reading frames are added, while to get the latter code the codons resulting from transversions in the first and third nucleotide bases of each codon are added.

We hypothesize that conserved proteins will contain sequences enriched in RNY codons or in the codons of the Extended Codes proposed.

In order to test this hypothesis and the putative existence of the intermediate genomes obeying either our Extended RNA Code Type I or II, we constructed sequences from the genomes of Streptococcus agalactie (A909, 2603 V/R) containing: a) RNY codons only, b) Codons pertaining to the Extended Code Type I, c) Codons pertaining to the Extended Code Type II. Utilizing these sequences we performed BLAST analysis to obtain fragments of the original genomes enriched in these specific codons.

We indeed obtained sequences of genes considered to be very ancient such as the corresponding tRNA's, ABC transporters, ATP synthase and some chaperones. These results support further the notion that there still remain vestiges of the RNA World in 
current genomes of bacterial organisms and there were at least two different evolutionary paths from the RNA code that led to the present SGC.

E-mail: marcojose@biomedicas.unam.mx

\section{On the Evolution of the Standard Genetic Code: From the RNA World to Current Prokaryote Genomes}

Marco V. José, Tzipe Govezensky, Juan R. Bobadilla

Theoretical Biology Group, Instituto deInvestigaciones Biomedicas, Universidad Nacional Autónoma de Mexico, Mexico D.F. 04510, Mexico

Herein two genetic codes from which the primeval RNA code could have originated the standard genetic code (SGC) are derived. One of them, called extended RNA code type I, consists of all codons of the type RNY plus codons obtained by considering the RNA code but in the second (NYR type) and third (YRN type) reading frames. The other one, called extended RNA code type II, comprises all codons of the type RNY plus codons that arise from transversions of the RNA code in the first (YNY type) and third (RNR) nucleotide bases. The former code specifies 17 amino acids, including AUG, the start codon, and the three known stop codons, whereas the latter code specifies 18 out of the 20 amino acids but no stop codons. In order to assess if both extended RNA codes, could be biologically meaningful, we used the whole genomes of four Eubacteria and two Archaeas, from which we obtained their respective genomes obeying the RNA code or the extended RNA code types I and II. We show that some symmetrical, statistical, and scaling properties of today bacterial chromosomes may be relic patterns of the primeval RNY genomes but mostly this is so for the extended RNA genomes. Remarkably, the scaling properties of the distance series of some codons from the RNA genomes and most codons from both extended RNA genomes turned out to be identical or very close to the scaling properties of the current bacterial genomes, but interestingly this is not so for Methanopyrus kandleri. To test for the robustness of these results, we show that random mutations at a rate of $10^{-10}$ per site per year during three billions of years of current genomes were not enough for destroying the observed patterns. Therefore, we conclude that current prokaryotes may still contain relics of the primeval RNA World and that both extended RNA codes may well represent two plausible evolutionary paths between the RNA code and the current SGC.

E-mail: marcojose@biomedicas.unam.mx

\section{Non-enzymatic Primer Extension Reactions: Stalling Factors for Mismatch Extensions and Misincorporations}

Sudha Rajamani ${ }^{1}$, Justin Ichida ${ }^{2}$, Doug Treco ${ }^{3}$, Tibor Antal $^{4}$, Martin Nowak ${ }^{4}$, Jack Szostak $^{3}$, Irene Chen ${ }^{1}$

${ }^{1}$ FAS Center for Systems Biology, Harvard University; ${ }^{2}$ Dept of Molecular and Cellular Biology, Harvard University; ${ }^{3}$ Dept of Genetics, Harvard Medical School; ${ }^{4}$ Program for Evolutionary Dynamics, Harvard University

The fundamental process by which living systems utilize and transfer genetic information is replication of nucleic acids and the transcription of DNA. Modern systems employ RNA and DNA enzymes to accomplish this important task. A more prebiotically relevant scenario 
would involve non-enzymatic, template-directed synthesis of complementary oligonucleotides from activated nucleoside 5 '-phosphates that are primarily catalyzed by polyribonucleotides and polydexyribonucleotides (Orgel and Lohrmann, 1974; Inoue and Orgel, 1982, 1983; Inoue et al. 1984; Acevedo and Orgel, 1987). The base sequence of the template essentially dictates the sequence to be synthesized. The presence of non Watson-Crick base pairs (mismatches) and misincorporation of the wrong nucleotide could have an important bearing on the rates of these non-enzymatic oligomerization reactions, as well as their error rates. Reaction rates would also be influenced by reverse hydrolysis reactions that could dramatically change the concentration of the starting components i.e. the template, the primer and activated monomers. Systematic studies have been undertaken to examine the accuracy of polymerization, catalyzed by an RNA polymerase ribozyme, by measuring the efficiency of matched and mismatched extension using four templates that differed only at the first coding nucleotide (Johnston et al. 2001). We sought to understand primer extension reactions that do not involve enzymes, which are prebiotically more relevant. We are currently determining mutation rates and the stalling factors for non-enzymatic extension reactions, by studying the effect of misincorporations as well as mismatches at the site of incorporation.

Acevedo, 0. L. and Orgel, L. E. (1987) Non-enzymatic transcription of an oligodeoxynucleotide 14 residues long. J. Mol. Biol., 197: 187-193.

Inoue, T. and Orgel, L. E. (1982) Oligomerization of (guanosine 5'-phosphor)-2methylimidazolide on poly(C): An RNA polymerase model. J.Mol. Biol., 162: 201-217.

Inoue, T. and Orgel, L. E. (1983) A Nonenzymatic RNA Polymerase Model. Science, 219: 859-862.

Inoue, T., Joyce, G. F., Grzeskowiak, K., Orgel, L. E., Brown, J. M. and Reese, C. B. (1984) Template-directed synthesis on the pentanucleotide CpCpGpCpC. J. Mol. Biol., 178: 669-676. Johnston, W. K., Unrau, P. J., Lawrence, M. S., Glasner, M. E., Bartel, D. P. (2001) RNACatalyzed RNA Polymerization: Accurate and General RNA-Templated Primer Extension. Science, 292:1319-1325

Orgel, L. E. and Lohrmann, R. (1974) Prebiotic chemistry and nucleic acid replication. Acc. Chem. Res., 7: 368-377.

E-mail: srajamani@cgr.harvard.edu

\section{Studies on the Activity of a Trans-acting Ribozyme in Hot Primordial Environments}

Giulia Talini, Sergio Branciamore, Enzo Gallori

Department of Evolutionary Biology, University of Florence, Italy

The hypothesis of a primeval RNA world is strongly affected by the hostile environmental conditions which were probably present on early Earth. In particular strong UV, X-ray radiations and high temperatures could have represented a major obstacle to the formation and evolution of the first genetic biomolecules.

With the aim at evaluating the possibility that a RNA world could have evolved in similar conditions, we studied the effect of one of these degrading agents, high temperatures, on the activity of a catalytic RNA molecule in three different environmental conditions: (1) Water solution ("the primordial broth"); (2) Presence of clay particles, montmorillonite, ("the mineral honeycomb"); (3) Presence of a dipeptide, Lys-Lys, to simulate a situation where both RNA-like molecules and aminoacids or short polypeptides could have been present at the same time. 
The preliminary results obtained suggest that RNA molecules maintain their ability to interact with each other and to undergo a trans-cleaving reaction either in presence of clay particles or Lys-Lys. Moreover, the presence of the dipeptide Lys-Lys seems to protect RNA molecules against high temperatures. The same protection was not found in presence of montmorillonite. The high stability of RNA/Lys-Lys could suggest that a crucial step for evolution towards a nucleosome-like structure was the interaction between first nucleic acid molecules and primordial peptides.

E-mail: giulia.talini@unifi.it

\title{
An RNA World Under Hydrothermal Environments on the Basis of Kinetic Analyses of the Prebiotic Formation of RNA
}

\author{
Kunio Kawamura, Jun Maeda, Hiroki Nagayoshi
}

Department of Applied chemistry, Graduate School of Engineering, Osaka Prefecture University

The discovery of catalytic RNA molecules has suggested that RNA or RNA-like molecules could have played a central role in the emergence of life on the primitive earth (Gilbert, 1986). This assumption has been experimentally verified by a number of successful studies on the condensation reactions of activated nucleotides to form RNA oligonucleotides in the presence of polynucleotide templates (TD reaction) (Lohrman and Orgel, 1980), metal ion catalysts (Sawai et al., 1989), or clay mineral catalysts (CL reaction) (Ferris and Ertem, 1992). However, the hypothesis that life originated under hydrothermal vent environments (the hydrothermal origin of life hypothesis) appears to be inconsistent with the RNA world hypothesis (Kawamura, 2004). According to the empirical data regarding the stability of RNA molecules, it is considered that the RNA molecules are too labile under redoxconstrained hydrothermal conditions (Anderson and Holm, 2000; Kawamura, 2003). Nevertheless, the prebiotic formation of RNA was rarely investigated at high temperatures.

Thus, we have accumulated kinetic data on the temperature dependence of prebiotic RNA polymerase model reactions, that is, the TD reaction (Kawamura and Umehara, 2001), $\mathrm{Pb}^{2+}$ ion-catalyzed oligonucleotide formation (PB reaction) (Kawamura and Maeda, 2007), and the CL reaction. These investigations suggested that its prebiotic formation could be faster than its degradation at high temperatures. In other words, it would be theoretically true that the accumulation of the RNA molecules can be kinetically controlled in an open system by both the formation and decomposition rates of RNA, even at high temperatures.

Besides, the biologically important interactions, such as hydrophobic interactions and hydrogen bonding, would not be effective at high temperatures. However, these interactions could not be experimentally verified at high temperatures. We have developed an in situ UVvisible spectrophotometer at high temperatures (Kawamura, 2002) and attempted to evaluate such interactions under hydrothermal conditions (Kawamura and Nagayoshi, 2007).

These facts imply that the RNA world hypothesis and the hydrothermal origin of life hypothesis could be compatible with each other.

Ferris, J. P. and Ertem, G. (1992). Oligomerization of ribonucleotides on montmorillonite: Reaction of the 5'-phosphorimidazolide of adenosine. Science, 257:1387-1389.

Gilbert, W. (1986). The RNA world. Nature, 319:618-618.

Kawamura, K. (2002). In situ UV-VIS detection of hydrothermal reactions using fusedsilica capillary tubing within 0.08-3.2 s at high temperatures, Anal. Sci., 18:715-716. 
Kawamura, K. (2003). Kinetics and activation parameter analyses of hydrolysis and interconversion of 2',5'- and 3',5'-linked dinucleoside monophosphate at extremely high temperatures. Biochim. Biophys. Acta, 1620:199-210.

Kawamura, K. (2004). Behavior of RNA under hydrothermal conditions and the origins of life, Int. J. Astrobiol. 3:301-309.

Kawamura, K. and Umehara, M. (2001). Kinetic analysis of the temperature dependence of the template-directed formation of oligoguanylate from the 5'-phosphorimidazolide of guanosine on a poly $(\mathrm{C})$ template with $\mathrm{Zn}^{2+}$. Bull. Chem. Soc. Jpn., 74:927-935.

Kawamura, K. and Maeda, J. (2007). Kinetic analysis of oligo(C) formation from the 5'monophosphorimidazolide of cytidine with $\mathrm{Pb}(\mathrm{II})$ ion catalyst at 10-75 C. Origins Life Evol. Biospheres, 37:153-165.

Kawamua, K. and Nagayoshi, H. (2007). Behavior of DNA under hydrothermal conditions with $\mathrm{MgCl}_{2}$ additive using an in situ UV-visible spectrophotometer. Thermochim. Acta, 466:63-68.

Lohrmann, R. and Orgel, L. E. (1980). Efficient catalysis of polycytidylic acid-directed oligoguanylate formation by $\mathrm{Pb}^{2+} . J$. Mol. Biol., 142:555-567.

E-mail: kawamura@chem.osakafu-u.ac.jp 


\title{
Early Biological Evolution
}

\section{Microbial Communities of Alkaline Hot Springs as a Model for Studying Early Stages of Biosphere Evolution}

\author{
Alla Brynskaya ${ }^{1}$, Oxana Pestunova ${ }^{2}$, Elena Lazareva ${ }^{3}$, Sergey Zhmodik $^{3}$ \\ ${ }^{1}$ Institute of Cytology and Genetics SB RAS, Novosibirsk, Russia; ${ }^{2}$ Boreskov Institute of \\ Catalysis SB RAS, Novosibirsk, Russia; ${ }^{3}$ Institute of Geology and Mineralogy SB RAS, \\ Novosibirsk, Russia
}

According to the hypothesis of the first Precambrian prokaryotic communities origin and development and their attendant environment (Zavarzin, 2004; Gerasimenko, 2004), chemical and gas composition and primary phototrophes structure of Barguzin valley hot springs in Baikal rift zone might represent analogs to relict Precambrian biocenoses. The research concerned microbial communities structure and composition, hot springs macroand microelements composition, minerals formed in microbial mat and a wide range of elements distribution between organic and mineral parts of mats.

Barguzin valley hot springs are alkaline siliceous hydrotermes with nitrogen prevailing in the gas. There were five springs studied at the right (Alla, Kuchiger, Umhei) and the left (Garga, Uro) sides of the valley; they differ a little in compound. The former ones have $\mathrm{SO}_{4}-$ $\mathrm{HCO}_{3}-\mathrm{Na}$ composition and contain $\mathrm{HS}^{-}$. The latter ones have $\mathrm{SO}_{4}-\mathrm{Na}$ composition. Do not contain $\mathrm{HS}^{-}$, but are characterized by a higher contain of radon. The communities developing in springs outlets are mostly represented by a cyanobacteria group, that is oxygenic phototrophic prokaryotes. Over all the time of their existence cyanobacteria had two quantity peaks: about two and one billion years ago. Bacterial mats mineral rests (stromatolites) form thick rock massifs. The spring communities with a higher sulfides contain possess a specific feature, i.e. a complex of dominants that could consist of diverse cyanobacteria species (Phormidium spp., Oscillatoria spp, Scytonema sp. and others), bacteria and algae. Here anoxygenic phototrophic bacteria Chloroflexus sp. or hemolitotrophic sulfur-reducing bacteria Thiothrix sp. can be labeled as active agents and edificators of the communities. In non-sulfide springs monodominant communities can be observed. These communities are represented by cyanobacteria Phormidium spp. or Mastigocladus laminosus, that formed thick gelatinous mats, in contrast to sulfide springs where mats were thin and easily destructible.

In cyanobacterial mats the precipitating of amorphous $\mathrm{SiO}_{2}$ and calcite has been determined. $\mathrm{SiO}_{2}$ depositions mainly occur as the result of the solution thermodynamic parameters changes. Calcite formation in a cyanobacterial mat looks like isometric (10$30 \mu \mathrm{m}$ ) crystals. There was found a direct relation between calcium contain in solution and calcite forming in mats. Microbial mats decisive role in large amount of elements accumulation has been determined. These elements are distributed in different ways between organic and mineral substance of the microbial mats. The distribution of $\mathrm{K}, \mathrm{Mn}, \mathrm{Ni}, \mathrm{Cu}, \mathrm{Zn}$, $\mathrm{Fe}$ is regular, $\mathrm{Ca}, \mathrm{Rb}, \mathrm{Sr}$ are almost totally related with the mats mineral part, while $\mathrm{Ga}, \mathrm{Ge}$ and $\mathrm{Br}$ are accumulated in mats organic substance. The microbial mats destruction does not entail $\mathrm{Ga}, \mathrm{Ge}$ and $\mathrm{Br}$ transformation into minerals, but results in their being carried away by water streams. Almost all the elements studied are accumulated by microbial communities. Exclusively in non-sulfide springs (Garga and Uro) Ge is accumulated by cyanobacterial mats.

Thus, basing upon studying of structure and some specific features of Baikal rift zone hydrotermes microbial communities functioning, it is possible to get a notion about the 
processes which occurred in Precambrian primary prokaryotic community and its significance for the modern biosphere formation.

This work was supported by the RFBR (06-05-64767, 06-05-64957, 08-05-00968); IP: 18-16 and 96; SS-5736.2008.5; RPN.2.1.1.702, PP SB RAS [2116]24 and Program "BOE".

Gerasimenko L.M., Orleansky V.K. (2004) Actualistic paleontology of cyanobacteria. In the same book: $80-108$.

Zavarzin G.A. (2004) Development of microbial communities in the Earth's history. Ed. by V.F Galchenko, Proceedings of Winogradsky Institute of Microbiology XII: 149-159. Moscow, Nauka.

E-mail: bal412003@mail.ru

\section{Prion: Catalyst for Biological Evolution}

\section{Jorge Enrique Bueno Prieto}

\section{Department of Biology, Universidad Nacional de Colombia, Bogotá, Colombia}

The theories of evolution of life on Earth have been based on the principle of storing and transmitting information of organisms in the genes, which according to the sequence of nucleotides could form proteins (Bowler 2003). This is so far the protocol of the evolutionary principle of life on Earth. This process since "not live" to "live" requires a neutral intermediary: the prion protein, or prion, is without cell and transferable skills these features make it to be an ideal candidate to be the first catalyst for life on our planet. More recent theories suggest that prions are proteins modified under certain circumstances such as changes in temperature, pressure or $\mathrm{pH}$ favored fall to a very stable energy level, allowing his return for three-dimensional conformation (Prusiner 1998).

The research aimed to describe the nature of prions and aggregates forming showed prion protein-organisms in their natural state, in a manner unrelated to illness (Weissmann 2004). Models in Fungi, particularly in Sacharomyces cerevisiae, have allowed observing the functions that could have prions in the life of normal cells. In these organisms, prions functions as the metabolic regulation of nitrogen. They also act as mechanisms of heredity phenotypes, in the role of evolutionary catalysts, and increasing genetic diversity by introducing new regions at the ends of the genome (i.e., Weissmann, et al. 2001).

The ability to store information conformational of prions makes them eligible to take part in cellular processes that require stability for long periods and it is possible that they are primitive cellular mechanisms.

It is likely that prions have been involved and participate in processes like the formation of the chemical long-term memory, immunological memory and evolution of the genome of many organisms (i.e., Farquhar, et al. 1983). Ultimately, prions are a means to update and transmit heritable characteristics confirmed that genes are not the only elements involved in inheritance and storage of information, so that while they do the genes in the genome, prions do so at of proteome for modifying an individual's life and transmit these characters acquired vertically and horizontally allowing the evolution of life (Shorter and Lindquist 2005).

Bowler, Peter J. (2003). Evolution:The History of an Idea. University of California Press. Farquhar C, Somerville R and Bruce M (1998). "Straining the prion hypothesis". Nature 391: 345-346. 
Prusiner SB (1998). "Prions". Proc. Natl. Acad. Sci. USA 95 (23): 13363-83

Shorter J, Lindquist S (2005). "Prions as adaptive conduits of memory and inheritance". Nat Rev Genet 6 (6): 435-50

Weissmann C, Enari M, Klöhn PC, Rossi D, Flechsig E (2002). “Transmission of prions". Proc. Natl. Acad. Sci. U.S.A. 99 Suppl 4: 16378-83.

Weissmann, C (2004). "The State of the Prion". Nature Reviews Microbiology 2: 861-871.

E-mail: jebuenop@unal.edu.co

\section{Why Does Symbiogenesis Impact the Origin of Life?}

Francisco Carrapio, Lusa Pereira, Telma Rodrigues

Universidade de Lisboa, Faculdade de Cincias, Departamento de Biologia Vegetal, Centro de Biologia Ambiental, Bloco C2, Campo Grande, 1749-016 Lisboa, Portugal

The main research lines on the origin of life have been based on classic Darwinian and neoDarwinian principles, and guided by the notion of evolution as a strictly competitive and gradual process, without any cooperative or synergistic involvement.

Usually, we consider Aleksandr Oparin and John Haldane's ideas as the main sources for the development of the modern thinking on the origins of life but, in 1909, Constantin Merezhkowsky pointed out the importance of extremophiles and extreme environments in early stages and evolution of life on Earth and introduced the symbiogenesis concept. Merezhkowsky defined it as "the origin of organisms through the combination or association of two or more beings that enter in symbiosis" (Sapp et al., 2002). According to this concept, symbiogenesis should be understood as an evolutive mechanism and symbiosis as the vehicle, through which that mechanism unfolds. This represents a different point of view from neo-Darwinism or the Modern Synthesis Theory, and the consideration of symbiosis takes studies of evolution onto a post neo-Darwinian level. These new ideas pointed out the central role of interactions, in which a new entity emerges through incorporation of one existing entity into another. It involves horizontal mergers, which can be rapid, and usually discontinuous, creating permanent and irreversible changes, the basis of evolutive novelty (Dyson, 1985; Carrapio et al., 2007).

The symbiogenic concept allows an innovative and a broader approach to the origin of life and evolution, given that symbiosis is a fundamental rule in the establishment and development of life on Earth and elsewhere (Carrapio et al., 2007). It implies a new paradigm for the comprehension of chemical and biological evolution. This change can be explained by a synergistic integrated cooperation between organisms, in which symbiosis acts, not as an exception, but rather as a rule in nature. According to these ideas, a symbiogenic approach to the pre-biotic evolution and origin of life should be seriously considered and developed as a new paradigm shift on evolution. We believe that cooperative, synergistic and communicational processes were responsible, using terrestrial and extraterrestrial materials, for the emergence of a large pre-biotic pool, closely related to geochemical and environmental conditions, and with intense interactions within.

We envision life's appearance accomplished through multiple origins, in different times and environments, displaying a variety of selective contexts, which optimized symbiogenic processes in the promotion of creative novelty. In this context, competition and cooperation can co-exist in the same scenario of evolution, and also at different 
population levels, which probably takes place in discontinuous bursts of activity, depending on internal and external conditions during the time and space of a hypercycle evolutive scenario. This Symbio-Darwinian approach enriches the models of life's appearance and development on Earth and beyond, with direct consequences in the construction of the astrobiological knowledge.

Carrapio, F., Pereira, L. and Rodrigues, T. (2007). Contribution to a Symbiogenic Approach in Astrobiology, Proc. of SPIE, 6694: 669406-1-669406-10.

Dyson, F. (1985) Origins of Life. Cambridge University Press, Cambridge.

Sapp, J., Carrapio, F. and Zolotonosov, M. (2002). Symbiogenesis: The Hidden Face of Constantin Merezhkowsky. Hist. Phil. Life Sci., 24: 413-440.

E-mail: fcarrapico@fc.ul.pt

\section{Giant Vesicles and w/o Emulsions as Biochemical Reactors}

P.Carrara, P. Stano, P. L. Luisi

Biology Dept. University of RomaTre, Rome

Giant vesicles (GVs) and w/o emulsion are micrometer-sized compartments which can be used to construct biochemical reactors. Such structures may be used as cell model to investigate foundamental properties of simple cells and protocells.

In this contribution, we will show how to use w/o emulsion to construct synthetic compartments. In particular, it will be shown a reactor that hosts a complex biochemical reaction inside (the expression of a protein) and simultaneously divides thanks to the increase of boundary surface (Fiordemondo and Stano, 2007).

Moreover, w/o emulsion can be used to construct GVs. Inspired by previously reported studies (Pautot et al., 2003; Noireaux and Libchaber, 2004) we have started a systematic investigation of GVs formation starting from the corresponding w/o droplets, at the aim of improving the reproducibility of the method and the capacity of sustain compartmentalized enzymatic reactions.

Fiordemondo D, Stano P (2007) Lecithin-based water-in-oil compartments as dividing bioreactors. ChemBioChem 8, 1965.

Noireaux V, Libchaber A (2004) A vesicle bioreactor as a step toward an artificial cell assembly. PNAS 101, 17669.

Pautot S, Frisken BJ, Weitz DA. (2003) Engineering asymmetric vesicles.

PNAS 100, 10718.

E-mail: pcarrara@uniroma3.it

\section{The World of the "Never Born Proteins"}

Chiarabelli C. ${ }^{1,2}$, De Lucrezia D. ${ }^{2,1}$, Stano P. ${ }^{1,2}$, Luisi P.L. ${ }^{1}$

${ }^{1}$ Departement of Biology, University of Roma TRE, Rome, Italy; ${ }^{2}$ ECLT, European Center for Living Technology, Venice, Italy

The rationality behind our research relies on the observation that the number of natural proteins on our Earth, although apparently large, is only a tiny fraction of all the possible 
ones. Indeed, there are thought to be roughly $10^{12-14}$ proteins of all sizes in extant organisms. This apparently huge number represents less than noise when compared to the number of all theoretically different proteins. This means that there is an astronomically large number of proteins that have never been sampled by natural evolution on Earth: the "Never Born Proteins" (NBPs). Exploration of random protein space is a relatively commonplace to search for novel pharmaceutical or to re-design natural enzymes with altered catalytic and kinetic properties. However, randomization is usually performed on a restricted region of target proteins, whereas the rest of it is left unchanged. Alternatively, a natural protein is used as a scaffold to engraft short random peptides. This approach can be defined as "directed randomization", since randomization is confined to a certain region in order to achieve a novel—yet, chosen 'a priori'—property.

The novelty in our research is basically different from "directed randomization" since it aims to explore the space of sequences of completely random proteins with no preconception as to what their properties might be: a "total randomization" approach.

With our work, using the technique of phage display, we were able to produce large libraries of random de novo polypeptides and identify sequences for further structural investigation. These NBP has totally random sequences, except for a tri-peptide (PRG) which is the site of thrombin cleavage-based on the consideration that folded proteins were protected against such a digestion. Our data show that, very surprisingly, the frequency of fold in such libraries of never born proteins is very high, about $20 \%$ of the entire set. The determination of the optimal substrate (PRG) for thrombin cleavage was of particular importance. Furthermore, and most importantly for the general philosophy of the concept, protein folding appeared to protect the PRG site against thrombin digestion, in both the phage-linked form as well in the free protein used as control.

This generalized protocol for the selection of folded proteins by proteolysis guarantees an efficient digestion of unstructured protein sequences while folded proteins are not affected. This procedure can be applied both for protein stabilization or selection of stable variants derived from a mutant library of extant proteins and for the selection of folded and stable sequences from de novo totally random phage libraries based on their fold properties.

The detailed structural study of each isolated protein is lengthy and complex and the characterization of purified samples is rate-limiting. In this preliminary phase, we present the partial characterization of few proteins, whereby the clones were chosen purely by a random procedure, which imparts a good degree of statistical validity despite their small number. In addition, the sequences have no putative conserved domains and no significant similarity with known protein sequences present in data banks. The sequences analysed in more detail appear to form globular, folded structures and, judging from the spectroscopic data (CD and fluorescence) and computer modelling they do not, at first sight, present peculiar structural features with respect to extant proteins.

More in general, all these data on NBP bear on a fundamental question: whether, and to what extent, extant proteins are the result of an obligatory evolution pathway (proteins had to be this way, or otherwise there would be no life) or, alternatively are they the product of contingency (they just happen to be this way and they permitted life by "chance")?

E-mail: c.chiarabelli@uniroma3.it 


\title{
The Origin and Evolution of Nitrogen Fixation Genes
}

\author{
Matteo Brilli ${ }^{1}$, Marco Fondi ${ }^{2}$, Pietro Liò ${ }^{3}$, Renato Fani $^{2}$ \\ ${ }^{1}$ Biometrie et Biologie Evolutive, UMR CNRS 5558, Université Lyon 1, Villeurbanne \\ Cedex, Lyon, France; ${ }^{2}$ Dept. of Evolutionary Biology, University of Florence, Italy
}

The ability to fix nitrogen relies on the activity of a set of nitrogen fixation (Nif) proteins, which have been particularly studied in the enterobacterium Klebsiella pneumoniae where 21 nif genes have been identified. It has been suggested that $\mathrm{N}_{2}$ fixation is an ancient biological process, which originated in the early stages of molecular evolution. In spite of the large body of information available for the genetic, biochemistry, and physiology of this process, little is known about the molecular mechanisms responsible for shaping nif genes and/or driving the assembly of nif metabolic pathway. To shed some light on this issue, the amino acid sequence of each of the $21 \mathrm{~K}$. pneumoniae Nif proteins was used to retrieve homologs from a set of 55 completely sequenced genomes including all diazotrophs species (30) and a representative set of other prokaryotic genomes. A non-redundant dataset of 4,200 proteins was constructed considering all hits with a Blast e-value below 0.0001; sequences were clustered using Blast2Graph (Lio' et al., 2008), a program for sequence clustering implementing the Markov clustering algorithm (Van Dongen, 2000). Data obtained can be summarized as follows:

(1) Four Nif proteins, that is NifW (NifO), NifT (FixU), and NifQ do not have paralogs. Besides, these sequences are also missing from about half of the diazotroph genomes analyzed and might represent optional genes for nitrogen fixation. (2) Eight Nif proteins (NifA, F, H, J, L, M, S, U) are related to proteins involved in other metabolic pathways (Out-paralogs). NifS is related to some proteins involved in amino acid and/or carbon metabolisms. NifJ, a multidomain Pyruvate:ferredoxin (flavodoxin) oxidoreductase, is part of a large multigene family whose representatives are involved in different metabolic processes. However, it is possible that NifJ is required for nitrogen fixation only in some diazotrophs (e.g. Erwinia carotovora), because orthologs are not easily identifiable in several species. Several proteins involved in Fe-Mo cofactor biosynthesis have paralogs in other similar processes, suggesting an ancestral interconnection between them. (3) Eight Nif proteins share a significant degree of sequence similarity with other proteins involved in nitrogen fixation or other metabolic routes (In-Out-paralogs). This group can be further split into two different clusters, the first one including NifD, K, E, N, and the second NifB, X, Y, V. As expected, proteins belonging to the first group showed a high degree of sequence similarity with Bch proteins involved in the biosynthesis of bacteriochlorophyll, supporting the idea of a common origin of these processes. NifB, X, and Y share a common domain of about 90 amino acid; moreover, NifB has an additional SAM domain, found in proteins that catalyze diverse reactions. Similarly, NifV proteins cluster with metabolic proteins, such as Isopropylmalate synthase.

Data obtained revealed that different molecular mechanisms might have shaped nitrogen fixation. In some cases it can be suggested that nif genes might have been recruited from other metabolic pathways, whereas the origin of other ones remains mysterious.

Stijn van Dongen. A cluster algorithm for graphs. Technical Report INS-R0010, National Research Institute for Mathematics and Computer Science in the Netherlands, Amsterdam, May 2000. 
Lio' P, Brilli M, Fani R (accepted BIRD 2008 conference). Topological Metrics in Blast Data Mining: Plasmid and Nitrogen-Fixing Proteins Case Studies. Lecture Notes in Computer Science, Springer

E-mail: renato.fani@unifi.it

\section{Hydrogen and Metal Catalysts in the Initiation and Early Evolution of Life}

\section{Mikhail Fedonkin}

\section{Paleontological Institute, Russian Academy of sciences, Moscow}

Most of known enzymes contain the transition metal ions as a cofactor of their active sites. These metalloenzymes loose their catalytic activity when the metal ions are being removed from the protein molecule. These facts indicate to the primary role of the metals in the origin of biocatalysis. Taxonomic distribution of the metalloenzymes gives a hint on the biogenesis as well. For example, the tungsten enzymes are discovered so far in prokaryotes only. However, obligatory dependence on tungsten is documented merely for hyperthermophylic Archea. Their basal position on the molecular tree of life points to the W-rich hydrothermal systems as a cradle of life. But the major catalysts on the earliest stages of the biogenesis were iron and nickel. The fact that nickel makes 5$20 \%$ of the iron meteorites indicates that both metals were abundant on young Earth. At present iron and nickel are actively involved in hydrogen metabolism which plays a key role in the prokaryotic and even eukaryotic organisms: virtually all hydrogenases contain $\mathrm{Fe}$ and/or Ni cofactor. This should turn our attention to the role of hydrogen in biogenesis. Hydrogen, the most abundant element in the Universe, well could be the primary fuel for early life. The availability of hydrogen on early Earth was much higher than at present. Two major sources of hydrogen were (1) the degassing of the mantle that released the neutral or slightly acidic fluids saturated with $\mathrm{H}_{2}, \mathrm{CH}_{4}$ and $\mathrm{H}_{2} \mathrm{~S}$, and (2) the serpentinization, reaction of the rocks, rich with olivine and pyroxene, with water. Two additional processes, such as photolysis of water by UV light and radiation-induced dissociation of $\mathrm{H}_{2} \mathrm{O}$ could contribute to the hydrogen budget as well. Abundance of hydrogen on early Earth gave easy access to the protons and electrons, the very motor of the life's energy machine. The subsequent evolution of life was in a great extent driven by the competition for access to hydrogen. Decline of the primary sources of hydrogen mentioned above made life to switch for the hydrogen compounds such as $\mathrm{H}_{2} \mathrm{~S}, \mathrm{CH}_{4}, \mathrm{NH}_{3}$, and at last, $\mathrm{H}_{2} \mathrm{O}$ in the oxygenic photosynthesis. The succession and degree of involvement of these simple molecules into early metabolic evolution could correlate to the energy required for breaking their chemical bonds in the conditions of early Earth. This concept helps to understand the historical causes of the atmosphere chemistry, in particular, the high content of nitrogen and oxygen as the byproducts of hydrogen metabolism. Early kinds of biochemistry, once established, have been saved throughout of the later history of life via addition of complementary metabolic modules in respose to the irreversible changes of the environment. This was the major driving factor of evolution towards the higher biological complexity.

Fedonkin, M. A. (2008), Ancient biosphere: The origin, trends and events. Russian Journal of Earth Sciences, 10, ES1006, doi:10.2205/2007ES000252. 
Hengeveld, R. and Fedonkin, M. A. (2007) Bootstrapping the energy flow in the beginning of life. Acta Biotheoretica, 55: 181-226.

E-mail: mfedon@paleo.ru

\title{
Unevolved Proteins from a Model Synthetic Proteome Are Functionally Active in vivo
}

\author{
Michael A. Fisher, Luke H. Bradley, Sara R. Viola, Michael H. Hecht
}

The polypeptides comprising the evolved proteomes of modern-day organisms are adequately functional macromolecules. The goal of our work is to assess the functional activity of unevolved protein sequence space-polypeptides that have not undergone evolutionary selection. To this end, we have used the binary code strategy for protein design to generate a large and combinatorially diverse collection of synthetic proteins. The binary code strategy for protein design enables the construction of synthetic libraries of folded proteins by specifying the locations of polar and nonpolar amino acids along a polypeptide chain in accordance with the periodicity of a desired element of secondary structure (alpha helix or beta sheet). However, because the binary code does not explicitly specify the identities of each polar or nonpolar side chain, this strategy facilitates enormous combinatorial diversity. Our target protein structure is a 102-residue four-helix bundle and the library is constructed at the gene level. We cloned our library of fully-assembled synthetic genes into an expression vector, generating a library of approximately $1.5 \times 10^{6}$ clones. To assess the functional potential of this model synthetic proteome, we tested whether de novo proteins from our library can provide biological activities essential for cell growth. We expressed the library of synthetic proteins in a series of $E$. coli single gene deletion strains that form colonies on rich media but not on M9-glucose minimal media (conditional auxotrophs). We have confirmed that deletions of several conditionally essential genes are compensated by the activity of de novo proteins from the library - the expression of certain synthetic proteins promotes colony formation in select $E$. coli conditional auxotrophs. These proteins do not bear significant sequence similarity to naturally occurring proteins, are $\alpha$-helical, as per our binary code design strategy, and are extremely thermostable. Our work demonstrates that even de novo polypeptides are genuinely poised for biological action and that unevolved proteins from a binary coded combinatorial library will readily promote life.

E-mail: mafisher@princeton.edu

\section{Origin of Plant Phenylalanine Ammonia Lyase: A Key Event for Land Colonisation?}

\section{Marco Fondi ${ }^{1}$, Giovanni Emiliani, ${ }^{2}$ Simonetta Gribaldo $^{3}$, Renato Fani ${ }^{1}$}

${ }^{1}$ Department of Evolutionary Biology, University of Florence, via Romana 19, 50125 Florence Italy; ${ }^{2}$ Department of Environmental and Forestry Technologies and Sciences, University of Florence, via S. Bonaventura 13, 50145 Florence, Italy; ${ }^{3}$ BMGE Unit, Pasteur Institute, 75724 Paris, France

Between 480 and 360 million years ago, land plants (Embryophytes) evolved, from the Charophyceae, a small group of freshwater green algae (Kenrick and Crane,1997), differentiating from simple structure (Bryophyte) to elaborate organisms showing an extraordinary array of complex organs and tissue systems (vascular plants).

However, in the first stages of prototrophs terrestrialization, beneficial associations between fungi (mycorrhizal symbioses), and soil bacteria $\left(\mathrm{N}_{2}\right.$ fixing), might have greatly 
helped early land plants to face a harsh environment characterised by important stresses including desiccation, UV radiation, and microbial attack (Selosse and Le Tacon, 1998).

A key event for plants colonisation of land and diversification was probably represented by the molecular evolution of phenylpropanoid pathway, since these compounds are involved in many stress response pathways (pathogens, grazing, ROS scavenging, UV screening, etc) as well as in other fundamental traits such as biosynthesis of lignin, the structural polymer able to guarantee stem rigidity and xylem (water conducting tissue) formation (Ferrer et al., and reference therein).

Despite its importance, the origin and evolution of the phenylpropanoid pathway, as well as the first advantageous physiological roles of its products are unclear.

Phenylalanine Ammonia Lyase (PAL) is responsible for the first committed step of plant phenylpropanoid pathway and the complete metabolism appears to be a specific and ubiquitous feature of land plants. However, PAL homologues have been identified and characterized in fungi such as Aspergillus oryzae (Seshime et al., 2005). Although phenylpropanoids are largely absent in prokaryotes, PAL homologues have been recently identified in Streptomyces maritimus and Photorhabdus luminescens where they are involved in the production of antimicrobial compounds (Xiang and Moore, 2005). A PAL homologue was also recently discovered in two cyanobacterial species of the order Nostocales (Moffit, 2007). PAL is homologous to Histidine ammonia lyase (HAL), which is involved in histidine degradation and it is present in prokaryotes and eukaryotes. It is thus commonly suggested that PAL evolved from HAL in fungi and plants (Boudet, 2007).

To shed some light on these issues, we have carried out an extensive phylogenetic analysis of PAL and HAL homologues.

The phylogenetic data lead us to propose a new evolutionary scenario involving two horizontal gene transfers: PAL originated in soil bacteria with an antimicrobial role, and was transferred (possibly from Nostocales species) very early to fungi via lichen-like symbioses and then to early land plants via ancient arbuscular mycorrhyzal symbioses, enabling the further development of the phenylpropanoid pathway and the radiation of plants on land.

Boudet (2007) Evolution and current status of research in phenolic compounds. Phytochemistry 68:2722-2735.

Ferrer, J.-L., Austin, M. B., C. Stewart Jr., C., and Noel J.P. Structure and function of enzymes involved in the biosynthesis of phenylpropanoids. Plant Physiology and Biochemistry 46:356-370.

Kenrick, P. and Peter R. Crane P. R. (1997) The origin and early evolution of plants on land. Nature 389:33-39.

Moffitt, M. C., Louie, G. V., Bowman, M. E., Pence, J., Noel, J. P. and Moore, B. S. (2007) Discovery of Two Cyanobacterial PAL: Kinetic and Structural Characterization. Biochemistry 46:1004-1012.

Selosse, M-A. and Le Tacon, F. (1998) The land flora: a phototroph-fungus partnership? Tree 13(1):15-20

Seshime, Y., Juvvadi, P. R., Fujii, I. and Kitamoto, K. (2005) Genomic evidences for the existence of a phenylpropanoid metabolic pathway in Aspergillus oryzae. Biochemical and Biophysical Research Communications 337:747-751.

Xiang, L. and Bradley S. Moore, B. S. (2005) Biochemical Characterization of a Prokaryotic Phenylalanine Ammonia Lyase. Journal Of Bacteriology 187(12): 4286-4289.

E-mail: marco.fondi@unifi.it 


\title{
Protolife in Precambrian Shadowed Fumaroles on the Moon
}

\author{
Jack Green
}

Department of Geology, California State University, Long Beach California State University, Long Beach, Long Beach, California, 90840 (562) 985-4198, Fax (562) 9858638

Lunar volcanism is presumed to have been extreme in the Hadean, as well as regional compared with a later Benioff-style of terrestrial volcanism which is suture controlled. A transient and tenuous lunar atmosphere is possible in the Hadean especially in the vicinity of fumaroles in topographic lows. Even today at Aristarchus, transient argon and radon gases have been detected at lunar sunrise.

Shadowed Precambrian lunar fumarolic fluids contain the ingredients for protolife. For example, in shadow neither formaldehyde, ammonia, nor methane will photodecompose. On earth at the submarine Lost City fumaroles, Proskurowski, et al. (2008) believe that abiogenic processes create long chain hydrocarbon precursors of protolife. There, as well as on the moon, Fischer-Tropsch reactions appear to not only convert fumarolic hydrogen, carbon monoxide and carbon dioxide into hydrocarbons but also create lipids. Lipid micelles, acting as reaction chambers, would prevent dilution and enhance concentration of pre-biotic lunar compounds. Most of these fluids in lunar shadow (40 K) would freeze and if over a centimeter thick most would persist over geologically long time periods because of their low vapor pressures. Hadean and later fumarolic fluids are believed to include ammonia, ammonium cyanide, carbon disulfide, carbon monoxide, carbon dioxide, carbonyl sulfide, chlorine, cyanogen, hydrogen sulfide, methane, nitrogen, sulfur, sulfur monochloride and water. According to O'Hara (2000) water would be required in the differentiation of the lunar highlands. Also the Apollo 17 colored volcanic spherules returned from the moon have oxygen fugacities as high as terrestrial volcanic glasses $\left(10^{-9} \mathrm{Po}_{2}\right)$ suggesting that lunar vent magmas were not as "dry" as the oft-quoted $10^{-13} \mathrm{Po}_{2}$ figures suggest.

Fumarolic compounds contain relatively high concentrations of both tungsten and soluble polyphosphates; the former acting as a critical metalloenzyme and the latter creating oligomeric amino acids leading by multiple steps to adenosine triphosphate and pre-RNA molecules. It has long been recognized that electrical energy from flow charging in volcanic vents and charge separation on freezing can both create many organic compounds including amino acids, formaldehyde and glycolaldehyde under reducing conditions. Amino acids and its products (with cyanide) can assemble into initially racemic proteins as membrane components. Ribose can also be formed and possibly stabilized by boron in fumarolic fluids. D-ribose, purines and polyphosphates may have led to pre-RNA replicating polymers to RNA to DNA possibly involving a bridging medium of methyl-RNA (Poole, et al, 2000). Montmorillonite and kaolinite as hydrothermal clays in fumaroles have been suggested as metabolic platforms for protolife including polymerization of peptides and oligonucleotides (Fishkit, 2007). Another positively charged biofilm platform is pyrite. The conversion of troilite - the most common lunar sulfide - with hydrogen can produce pyrite with a thermodynamically viable negative free energy of $-41.9 \mathrm{~kJ} / \mathrm{mol}$ (Wächterhauser, 1988). Other simple combinations of troilite, hydrogen sulfide and carbon dioxide with negative free energies can produce methylthiols as well as a colloidal pyritic biofilm to which organic molecules could attach and receive energy.

There are many stimuli for the origin of protolife in all types of Hadean and later fumaroles. From the vent outwards we see: (1) abrupt environmental changes and zonation over distances of 
meters $(\mathrm{eH}, \mathrm{pH}$, freeze/thaw, fluctuating clay environments including homoionic montmorillonite, conductivity), (2) possible proflavin (PAH) for nucleotide assembly into RNA (3) flash evaporation and (4) lower surface pressures. Lower surface pressures would greatly reduce the boiling points of fumarolic fluids and produce larger bubbles extending the vapor phase range of lunar protolife compounds enhancing reactivity. Reactivity would also be increased by convection, reflux and fluidization in fumarolic vents.

One of the more interesting stimuli for Precambrian lunar protolife is fumarolic spatter and wet/dry cycles, combined with lower lunar gravity and surface pressure (Green, 1965). Spatter of particles $0.1 \mathrm{~m}$ or less from fumaroles on earth at Kuirau Park in Rotorua in New Zealand on January 26, 2001 were thrown $100 \mathrm{~m}$. On the moon, this would produce a spatter blanket of some one million square meters. Spatter would have relatively high concentrations of nucleotides, catalysts, enzymes and divalent cations By flash evaporation hot lunar spatter landing on montmorillonite could possibly produce pyrimidines including cytosine on dryout (Nelson, et al. 2001) as well as ammonium cyanide. Drying and heating in fumaroles could possibly promote polymerization reactions of oligonucleotides and peptides. Wet/dry cycles of clay-rich vents have been shown to produce peptides of 12 to 20 amino acids chains (Penny, 2003).

Also modifying the arguments of Lathe (2004) for the origin of life in rapid terrestrial ocean tidal cycles, a version of a polymerase chain reaction favoring double strand RNA or DNA replication and amplification might relate to lunar fumaroles during wet and dry cycles. During the drying phase of fumarolic spatter cycles, characterized by high soluble cation concentrations, the opposing $\mathrm{PO}_{4}$ groups that separate each sugar nucleotide monomer in double stranded RNA or DNA would be more effectively neutralized by divalent fumarolic ions $\left(\mathrm{Mg}^{+2}, \mathrm{Ca}^{+2}, \mathrm{Ba}^{+2}\right.$ ) (versus Lathe's monovalent ion terrestrial model); interstrand hydrogen bonding would promote association of the two polymer strands favoring RNA/DNA replication. Copying by the RNA/DNA polynucleotide can only take place during the drying phase along with non-enzymatic polymerization through dehydration condensation.

Finally, potential fumarolic sites on the moon (Green, 2007) would be covered by unknown thicknesses of impact and volcanic ejecta. Fumarolic protolife, if present, would probably occur in disseminated ices, in ice lenses or in clathrates.

Blank, J. (2005). Earth's primitive environment and exogenous sources of ingredients for prebiotic chemical evolution. (Abstract), Orig. Life Evol. Biosphere, 36: 204

Fishkit, M. (2007). Steps toward the formation of a protocell; the possible role of short peptides. Orig. Life Evol. Biosphere, 37: 537-553

Green, J. (1965). Tidal and gravity effects intensifying lunar defluidization and volcanism. Annals N.Y. Acad. Scis., 123: 403-469

Green, J. (2007). Implications of a caldera origin of the lunar crater, Copernicus. EOS Trans. AGU, 88, Fall Meeting Supplement,

Abstract P41A-0227 and poster.

Lathe, R. (2004). Fast tidal cycling and the origin of life. Icarus, 168: 18-22

Monnard, P.A. and Szostak, J.W., (2008). Metal-ion catalyzed polymerization in the eutectic phase in water-ice: A possible approach to template-directed RNA polymerization. Jour. Inorg. Biochem., 102: 1104-1111

Nelson, K.E., Robertson, M.P., Levy, M. and Miller, S.L. (2001). Concentration by evaporation and the prebiotic synthesis of cytosine.

Orig. Life Evol, Biosphere, 31: 221-229

O'Hara. M.J. (2000) Flood basalts, basalt floods or topless Bushvelds?: Lunar petrogenesis revisited. Jour. Petrology, 41: 1545-1651 
Poole, A.M., Penny, D. and Sjoberg, B-M. (2000). Methyl-RNA: Evolutionary bridge between RNA and DNA. Chemistry and Biology, 7:R207-R216

Proskurowski, G., Lilley, M.D., Seewald, J.S., Früh-Green, G.L., Olson, E.J., Lupton, J.E., Sylva, S.P., and Kelley, D.S. (2008).

Abiogenic hydrocarbon production at Lost City hydrothermal field. Science 319: 604-607 Ryder, G., (2003). Bombardment of the Hadean Earth: Wholesome or deleterious? Astrobiol., 3: 3-6

Wächterhäuser, G. (1988). Before enzymes and templates; Theory of surface metabolism. Microbiological Reviews, 52: 452-484

E-mail: jgreen3@csulb.edu

\section{Horizontal Transfer of Archaeal Eocyte Ribosomal RNA Genes}

Craig Herbold ${ }^{2}$, Jacqueline Servin ${ }^{2}$, Ryan Skophammer ${ }^{1}$, James A Lake La,2, $^{1,2}$

${ }^{1}$ Department of MCD Biology, University of California, Los Angeles, CA 90095; ${ }^{2}$ Molecular Biology Institute, University of California, Los Angeles, CA 90095, USA; ${ }^{3}$ Department of Human Genetics, University of California, Los Angeles, CA 90095, USA

Small-subunit ribosomal RNA (SSU-rRNA) genes are generally assumed to be immune to horizontal transfer and therefore have been used extensively as a marker for reconstructing organismal phylogeny and in taxonomic classification. In the last decade, however, several reports have claimed to provide evidence of horizontal transfer of both large-subunit (LSU) and small-subunit (SSU) ribosomal RNA gene sequences (Yap, et al., 1999; Parker, 2001; van Berkum et al., 2003; Boucher et al., 2004; Miller et al., 2005). A common theme in these reports is that ribosomal RNA genes under the influence of HGT appear to exhibit genetic mosaicism. Small (50-300 nt) portions of an endogenous ribosomal gene appear to be displaced by corresponding segments from an exogenous source. These observations suggest that the detection of horizontal transfer of SSU-rRNA sequences may be readily accomplished by detecting recombination between SSU-rRNA sequences. We examined structure-based alignments for evidence of recombination between archaeal eocyte SSUrRNA sequences and found significant evidence of recombination. Recombination between archaeal eocyte SSU-rRNA genes can only be explained by invoking horizontal transfer because this group of taxa contains a single SSU-rRNA gene per genome. Recombination detected between archaeal eocyte SSU-rRNA genes provides convincing evidence that horizontal transfer does influence the evolution of this gene and should not be ignored. The resulting mosaicism in SSU-rRNA genes violates the phylogenetic assumption that this single gene corresponds to a single phylogenetic history. Due to this violation, prokaryotic classifications and relationships based on SSU-rRNA may need re-evaluated, especially the "deep" relationships between prokaryotic domains and phyla.

Boucher Y, Douady CJ, Sharma AK, Kamekura M, Doolittle WF. (2004) Intragenomic heterogeneity and intergenomic recombination among haloarchaeal rRNA genes.J Bacteriol., 186(12):3980-90.

Miller SR, Augustine S, Olson TL, Blankenship RE, Selker J, Wood AM. (2005). Discovery of a free-living chlorophyll d-producing cyanobacterium with a hybrid proteobacterial/cyanobacterial small-subunit rRNA gene., Proc Natl Acad Sci U S A., 102 (3):850-5. 
Parker MA. (2001). Case of localized recombination in 23S rRNA genes from divergent bradyrhizobium lineages associated with neotropical legumes. Appl Environ Microbiol., 67 (5):2076-82.

van Berkum P, Terefework Z, Paulin L, Suomalainen S, Lindström K, Eardly BD. (2003). Discordant phylogenies within the rrn loci of Rhizobia. J Bacteriol.,. 185(10):2988-98.

Yap WH, Zhang Z, Wang Y. (1999). Distinct types of rRNA operons exist in the genome of the actinomycete Thermomonospora chromogena and evidence for horizontal transfer of an entire rRNA operon. $J$ Bacteriol., 181(17):5201-9.

E-mail: cherbold@ucla.edu

\title{
The Role of Internal Gene Duplication in Protein Evolution
}

\author{
Ricardo Hernández-Morales, Arturo Becerra, Luis Delaye, Antonio Lazcano
}

Facultad de Ciencias UNAM, 04510, México, D.F.

A set of highly conserved sequences which are involved in RNA metabolism has been analyzed in order to assess the role of internal gene duplication events that may have taken place during early biological evolution. Our results show that some ancient sequences found in all three major cell lineages are the outcome of internal duplications followed by fusion events. The sequences which we have found are the outcome of internal duplication events include those related to major biological processes including transcription, translation, regulation and the biosynthesis and degradation of ribonucleotides, ribonucleotide-derivatives, and polyribonucleotides

E-mail: odracir6@gmail.com

\section{Requirements for Efficient Replication of Genetic Information in a Translation-Coupled Replication System}

Norikazu Ichihashi ${ }^{1}$, Hiroshi Kita ${ }^{2}$, Kazufumi Hosoda ${ }^{1}$, Takeshi Sunami ${ }^{2}$, Koji Tsukada ${ }^{3}$, Tomoaki Matsuura ${ }^{1}$, Tetsuya Yomo ${ }^{1,2,4}$

${ }^{1}$ Graduate School of Information Science and Technology, Osaka University; ${ }^{2}$ Complex Systems Biology Project, ERATO, JST; ${ }^{3}$ Graduate School of Applied biotechnology; ${ }^{4}$ Graduate School of Frontier Bioscience, Osaka University

The genetic information of all present-day living systems is replicated by a self-encoded replication enzyme, where two reactions, translation of replicase and replication of genetic information by the translated replicase, are required. These two reactions were separated temporally and spatially in present-day cells, whereas they are considered to be occurred at the same time in primitive living systems. In those primitive self-encoding systems, the two reactions can compete for the genetic information molecule because both reactions use the same information molecule as a template. Therefore, it is important to find the condition under which the primitive self-encoding system works efficiently for understanding of how the present-day sophisticated replication systems evolved. Recently, we reconstructed a self-encoding system for replication of genetic information (Kita et al. submitting), in which the catalytic subunit of $Q$ $\beta$ replicase, an RNA-dependent RNA polymerase originated from coliphage $Q \beta$, was translated from the sense strand RNA by a reconstituted translation system, resulting in synthesis of complementary strands of sense RNA to replicate the genetic information. The characteristic features of this system are non-linear dynamics of RNA replication and 
competition for the template RNA between translation and replication. Using this reaction system as an experimental model, we try to understand the dynamic behavior of the system quantitatively. We constructed a kinetic model which could describe the whole dynamic behavior of the self-encoding replication system. The results of this quantitative study indicated that the balance between translation and replication was critical for efficient self-encoding replication because of the inhibitory effects of translation on RNA replication. These results would deepen our understanding of how living systems evolve to be a sophisticatedly coordinated replication systems.

E-mail: ichihashi@ist.osaka-u.ac.jp

\title{
A Comparative Analyses of Different Methodologies Employed for the Reconstruction of the Gene Complement of the Last Common Ancestor
}

\author{
Sara E. Islas, Arturo Becerra, Luis Delaye, Antonio Lazcano*
}

Facultad de Ciencias UNAM, 04510, Mexico, D.F.

Although it is generally accepted that the last common ancestor (LCA, also referred to as LUCA) was a complex organism perhaps not so different from extant prokaryotes, there are different estimates of its gene complement. Here we report the outcome of a comparative analysis of the different methodologies that have been developed based on comparative genomics and phylogenetic analyses. The different estimates of the gene content of the LCA show an impressive overlap for a significant number of highly conserved sequences involved in basic biological processes. The core of highly conserved RNA-related sequences supports the hypothesis that the LCA was preceded by earlier entities

E-mail: saraernes@yahoo.com

\section{Random Sequence Polypeptides: A Model for Understanding the Origins of Natural Proteins}

A. Marcozzi ${ }^{1}$, C. Chiarabelli ${ }^{1,2}$, A. Quintarelli ${ }^{1}$, D. De Lucrezia ${ }^{2,1}$, P. L. Luisi ${ }^{1}$

${ }^{1}$ Department of Biology, University of RomaTre, Rome, Italy; ${ }^{2}$ ECLT, European Center for Living Technology, Venice, Italy

Our approach to the study of life is focused on finding a model that could explain the origin and evolution of the first proteins appeared on earth. In fact, such proteins may have been the result of simple condensation reactions of amino acids, these reactions were probably DNA independent and so their products were short random polypeptides.

Of course, similar molecules are far away from having the properties of enzymes but may have been the original population from which are then emerged the natural proteins. A characteristic certainly indispensable for the catalytic activity is the three-dimensional structure. From this evidence was born the idea that the folding could have been an important factor of discrimination between prebiotic polypeptides; chains able to have a stable fold are more soluble in water and more resistant to hydrolysis, have a greater "fitness" than other and could therefore have been naturally selected for this feature. For these reasons, our interest is focused on short random polypeptide sequences, these are in fact much more resemble natural proteins to those who may have been the first enzymes that were formed on our planet. 
To discriminate folded proteins against the unstable ones it was decided to subject the library of sequences produced by Phage Display to enzymatic digestion. The polypeptides were designed to contain in the middle of the random sequence the PRG residues, substrate recognized by the protease Thrombin. In this way it is possible to distinguish those proteins inside the library that are resistant to enzyme from those that are digested. The resistant proteins have probably a tertiary structure that makes the PRG site inaccessible to protease. The library was further tested by subjecting sequences of interest to other proteolytic non specific enzymes such as trypsin and chymotripsine. The activity of these proteases is influenced by the nature of tertiary structure of the protein substrate, therefore the analysis of the digestion products can highlight the formation of particularly stable structures. The interested polypeptides were subjected to enzymatic digestion for various time intervals and with different protease concentrations. Cyclical steps of this procedure were resulted to select, inside the library, the more resistant sequences, the ones that may to have a stable tertiary structure and thus may have potentially some kind of biological activity.

The investigation of 79 sequences, randomly selected from the initially large library, shows that over $20 \%$ of this population is thrombin-resistant, likely due to folding. Analysis of the amino acid sequences of these clones shows no significant homology to extant proteins, which indicates that they are indeed totally de novo.

The DNA sequences coding the corresponding resistant proteins were cloned into appropriate vectors, expressed in E. coli and then purified and analyzed in order to determine the tertiary structure and assess the chemical and physical characteristics.

A few selected polypeptides have been characterized by spectroscopic methods and, in particular, by circular dichroism. The data show a stable three-dimensional folding, which is temperature-resistant and can be reversibly denatured by urea. The consequences of this finding within a library of "Never Born Proteins" are discussed in terms of molecular evolution.

In addition, the polypeptide sequences resistant to proteolytic activity have undergone structure prediction by Rosetta method, the results showed the presence of secondary structures spread, mainly a-helices, and the formation of compact tertiary structures. The data will be confirmed by next structural analysis by X-ray diffraction.

The novelty of this work is to select completely new sequences that probably even nature has ever been able to face with. With this research we intend therefore to lay the groundwork for a totally new protein engineering, aiming to achieve polypeptides totally new, with no correlation with the existing proteins to investigate which new structures and activities can hide behind de novo random protein sequences.

E-mail: alessio.marcozzi@gmail.com

\title{
[FeFe] Hydrogenases: A Modern Bio-catalytic Link to Ancient Geochemistry
}

\author{
Shawn E. McGlynn, Eric Shepard, Shane Ruebush, Joan B. Broderick, John W. Peters*
}

Astrobiology Biogeocatalysis Research Center and the Department of Chemistry and Biochemistry, Montana State University, Bozeman, MT 59717

Iron sulfur minerals have been proposed to have a prominent role in the catalytic formation of molecules that eventually became integrated into biological systems (Russel, 2007). Iron sulfur enzymes, which exist as highly evolved mineral clusters, may provide clues to the potential emergence of biologically-relevant chemistry on mineral surfaces, existing as testaments to the efficacy of conducting organic chemistry at inorganic catalytic centers. Enzymes harboring 
distinct, ligand modified cofactors are especially of interest due to their resemblance to putative catalytic sites on minerals of the early earth; understanding routes to biological availability/ assembly of these clusters might provide insights as to the nature of recruitment of these mineral forms by biological systems. In this light, we are examining the structure, function, and overall assembly of the complex-iron-sulfur enzymes nitrogenases and hydrogenases. With regard to the latter we have been examining aspects of the biosynthesis of the active site, $\mathrm{H}$ Cluster, of $[\mathrm{FeFe}]$ hydrogenases, which exists as a $[4 \mathrm{Fe}-4 \mathrm{~S}]$ cluster linked via a cysteinyl thiolate to a two iron unit which is ligated by cyanide, carbon monoxide, and a unique bridging dithiolate (Peters, 2009). We have developed an in vitro activation scheme for heterologously expressed hydrogenases, and have furthered these observations in identifying a single specific scaffolding protein as being involved in this process (McGlynn et al., 2008). The results provide the basis for examining the relationship between the biochemical pathways for complex biological iron-sulfur cluster synthesis and related chemical modifications in ironsulfur minerals involved in prebiotic catalysis. With this knowledge, we are conducting hypothesis driven studies aimed at the elucidation of biochemical and evolutionary pathways in which biology developed this remarkable enzyme from geologic pre-cursors.

McGlynn, S. E., Shepard, E. M., Winslow, M. A., Naumov, A. V., Duschene, K. S., Posewitz, M. C., Broderick, W. E., Broderick, J. B., and Peters, J. W., 2008, HydF as a scaffold protein in [FeFe] hydrogenase H-cluster biosynthesis: FEBS Lett, v. 582, no. 15, p. 2183-7.

Peters, J.W., in "Metal-Carbon Bonds in Enzymes and Cofactors",

Vol. 6 of 'Metal Ions in Life Sciences'; A. Sigel, H. Sigel, R. K. O. Sigel, Eds.;

The Royal Society of Chemistry, Cambridge, UK, 2009, in press.

Russell, M. J., 2007, The alkaline solution to the emergence of life: Energy, entropy and early evolution: Acta Biotheoretica, v. 55, no. 2, p. 133-179.

E-mail: john.peters@chemistry.montana.edu

\title{
Emergence of Animals During Snowball Earths from Biological Heat Engines in the Thermal Gradient Above Submarine Hydrothermal Vents
}

\author{
Anthonie W. J. Muller
}

\section{Swammerdam Institute for Life Sciences, University of Amsterdam}

Previously a model has been given for the origin of life based on thermosynthesis, biological free energy gain from thermal cycling (Muller, 1995, 2005; Muller and SchulzeMakuch, 2006). Convection in volcanic hot springs drove a first protein (FP), the progenitor of the $\beta$ subunit of the $F_{1}$ moiety in today's ATP Synthase. This FP not only generated ATP (or NTPs) during thermal cycling, but also peptides, phospholipids and the phosphodiester bonds of RNA - which started the RNA World. The described emergence of a set of transfer RNA molecules is consistent with the phylogenetic tree obtained from extant transfer RNAs (Sun and Caetano-Anollés, 2008).

Here a thermosynthesis based model is proposed for the origin of animals as well. During global glaciations (Kirschvink, 1992) FPs were thermally cycled while attached to proteins that performed a relaxation oscillation in the thermal gradient above a submarine hydrothermal vent. The mechanisms involved denaturation of filamentous proteins or a temperature-controlled entry to a body cavity. As at low Reynolds number (Purcell, 1977) movements caused by thermal transitions are not hindered by friction, the machineries could start small and then increase in size. At the end of a glaciation, the emerged large 
machineries reversed upon symbiosis with the ATP-generating progenitors of today's mitochondrions: ATP was used to effect movement. The reversals yielded the coelom and the tentacle, key organs of the Ediacarans.

During the short glaciation at the start of the Cambrium, ATP-generating mechanisms emerged that worked on the protons released during high temperature biomineralization (McConnaughey and Whelan, 1997) and on the thermal diffusion potential (Agar, 1963); their reversal yielded the skeleton and the nerve. Using a scenario already proposed by Empedocles, the emerged single-organ organisms then formed by symbiogenesis (Margulis, 1981 ) the numerous multiple-organ animals (metazoans) of the Cambrian explosion.

Agar, J.N. (1963). Thermogalvanic cells. Advances in Electrochemistry and Electroengineering, 3:31-121.

Kirschvink, J.L. (1992). Late Proterozoic low-latitude global glaciation: the Snowball Earth. In Schopf, J.W. and Klein, C., editors, The Proterozoic biosphere: A multidisciplinary Study, pages 51-52. Cambridge University Press, Cambridge, UK.

Margulis, L. (1981). Symbiosis in cell evolution, Freeman, San Francisco, CA.

McConnaughey, T.A. and Whelan, J.F. (1997). Calcification generates protons for nutrient and bicarbonate uptake. Earth-Science Reviews, 42:95-117.

Muller, A.W.J. (1995). Were the first organisms heat engines? Progress in Biophysics and Molecular Biology, 63:193-231.

Muller, A.W.J. (2005). Thermosynthesis as energy source for the RNA world: A model for the bioenergetics of the origin of life. BioSystems, 82:93-102.

Muller, A.W.J. and Schulze-Makuch, D. (2006). Thermal energy and the origin of life. Origins of Life and Evolution of Biospheres, 36:177-189.

Purcell, E.M. (1977). Life at low Reynolds number. American Journal of Physics, 45:3- 11. Sun, F.J. and Caetano-Anollés, G. (2008). The origin and evolution of tRNA inferred from phylogenetic analysis of structure. Journal of Molecular Evolution, 66:21-35.

E-mail: a.w.j.muller@uva.nl

\section{Stromatolite of Possible Archean Age from Bundelkhand Craton, Central India}

\section{J. K. Pati*, G. Shukla, A. K. Rao, S. Yadav}

Department of Earth and Planetary Sciences, Nehru Science Center, University of Allahabad, Allahabad-211002, India

The Archean stromatolites are rare and reported from 48 locations from different parts of world with an age range between 2,500 and 3,500 Ma (Schopf et al. 2007). The present study reports the first occurrence of stromatolites in calc-silicate lithology (N 25 $18^{\prime} 14.9^{\prime \prime}$, E $78^{\circ} 05^{\prime}$ 32.2"; elevation: $312 \pm 10.9 \mathrm{~m}$ ) occurring $4.4 \mathrm{~km}$ WNW of Dhala, Shivpuri District, Madhya Pradesh State, India. The calc-silicate lithology occupies nearly $4.3 \mathrm{~km}^{2}$ area. The calc-silicate rocks form linear, low-lying, and blocky outcrops. It is intimately associated with diorite in the north, and intrusive micro-granites of its southern part. The calc-silicate rock is light greenish grey in colour with alternating moderate to dark bands of variable thickness and comprises quartz + hornblende + alkali feldspar + diopside \pm zircon \pm epidote \pm sericite \pm calcite \pm opaque. The stromatolite-bearing calc-silicate rock is older than the host granitoids $(2.5 \mathrm{Ga})$. It is interesting to note that, the stromatolite-bearing calc-silicate rock is one of the pre-impact rock types associated with a newly discovered Dhala impact structure $\left(\mathrm{N} 25^{\circ} 17^{\prime}\right.$ 59.7" and E $78^{\circ} 8^{\prime} 3.1^{\prime \prime}$ ) of Paleoproterozoic age (Pati 2005 and Pati et al., in press). 
The stromatolites mostly occur as flat-laminated mats and vary from flat-laminated to strongly convex type. The stromatolites can be classified as close laterally linked hemispheroid (LLH-C) type. Maximum and minimum thickness of laminaes is between 0.55 and $4.93 \mathrm{~mm}$, respectively. Laminaes are wavy in nature, show low synoptic relief and high inheritance. In profile section, the laminaes are gently convex. This finding has a tremendous bearing on the evolution of hitherto unknown early life forms in the Archean Bundelkhand craton vis-à-vis central Indian shield.

Pati, J. K. (2005). The Dhala Structure, Bundelkhand craton, Central India-a new large Paleoproterozoic impact structure (abstract), Meteoritics \& Planetary Science 40 (Supplement): A121.

Pati, J. K., Reimold, W.U., Koeberl, C. and Pati, P. (in press). The Dhala Structure, Bundelkhand Craton, Central India-eroded remnant of a large Paleoproterozoic impact structure. To appear in the Meteoritics \& Planetary Science.

Schopf, J. W., Kudryavtsev, A. B., Czaja, A. D., Tripathi, A. B. (2007). Evidence of Archean life: Stromatolites and microfossils. Precambrian Research, 158:141-155

E-mail: jkpati@yahoo.co.in

\title{
The Minimal Size of Cells: An Experimental Approach Based on Liposomes
}

\author{
Tereza Pereira de Souza ${ }^{1}$, Pasquale Stano ${ }^{1}$, Pier Luigi Luisi ${ }^{1}$
}

Biology Department, University of RomaTre, Viale G. Marconi 446; 00146 Rome, Italy

In the last few years the notion of the "minimal cell", as a form of minimal life, has gained considerable attention both from the theoretical and experimental point of views (Luisi, 2006; Luisi et al. 2006). This concept is important for assessing the minimal and sufficient conditions for cellular life, and also to gain an insight of the early cells, conceivably much simpler than the modern cells. There are two sides to the notion of minimal cell: one side is the question of the minimal genome, namely the minimal number of expressed genes that permit the functioning of the cell (usually seen in terms of the triad self-maintenance, reproduction, and evolvability). The other side to it concerns the minimal physical dimension of the cell the question, namely, on the dimension that still permits a cellular life. These two aspects minimal genome and minimal size are obviously connected to one another, being also related to evolutionary paths and to the environment composition. Here we propose to examine the question of the minimal physical size of cells by using liposomes with entrapped the complete ribosomal machinery for protein expression (enhanced green fluorescence protein, EGFP). Liposomes are formed by film or ethanol injection method. The synthesis outside vesicles was inhibited using the EDTA, RNAse or protease, with the inhibitor being added just after vesicles formation. The system with the addition of inhibitor inside and outside of vesicles formed our negative control. The EGFP fluorescence was followed in time, and the bright fluorescence observed in vesicles confirms that EGFP is expressed only inside vesicles. Concerning the minimal size, we observed that liposomes with radius ca. $100 \mathrm{~nm}$ were still capable of protein expression; furthermore, surprisingly enough, the efficiency was higher than in bulk water. In order to express the protein, the liposomes should contain all hundred or so molecular components. This proves to be a riddle, as classic statistical analysis would give zero or negligible probability to the simultaneous entrapment of so many different molecular components 
(Souza et al, submitted). The possible raisons of this challenging puzzle, possibly important for the origin of life scenario, are discussed.

\section{Financial Support}

T.P. Souza was supported by the CNPq Post-doctoral fellowship 210295/2006-6 (Brazil).

Luisi, P. L. (2006) The emergence of life: from chemical origins to synthetic biology. Cambridge University Press.

Luisi, P. L., Ferri, F., and Stano, P. (2006) Approaches to semi-synthetic minimal cells: a review. Naturwissenschaften, 93, 1-13.

Souza, T. P., Stano, P., and Luisi, P. L. (submitted) The minimal size of cells: an experimental approach based on liposomes.

E-mail: terezapsouza@yahoo.com.br

\section{A Genomic Approach to the Evolution of Metabolism: Convergence and Complementation in Insect Endosymbionts}

\section{J. Peretó, M.J. López-Sánchez, A. Lamelas, M.J. Gosálbez, A. Neef, R. Gil, A. Moya, A. Latorre}

Institut Cavanilles de Biodiversitat i Biologia Evolutiva, Universitat de Valencia

Comparative studies of insect-endosymbiont genomes have illuminated the metabolic adaptation to intracellular lifestyle (Moya et al. 2008). A high number of insect species have established a symbiotic relationship with bacteria. In general, such insects feed on unbalanced diets, which are supplemented by bacterial endosymbionts. Aphids and cockroaches are model systems to study the dependence of the metabolic evolution of endosymbiotic bacteria on the chemical composition of their diet.

Aphids are plant-sap feeding insects, a diet rich in carbohydrates but deficient in essential amino acids and vitamins that are supplied by the endosymbionts. In particular, Buchnera aphidicola BCc (a gamma-proteobacteria associated with the aphid Cinara cedri) possesses the smallest Buchnera genome, with only $422 \mathrm{~kb}$. Its functional analysis indicates that tryptophan and riboflavin should be supplied by another source. Thus, the secondary endosymbiont Candidatus Serratia symbiotica has been proposed to carry out this role (Pérez-Brocal et al., 2006). We have sequenced the genome of S. symbiotica using 454 technology, and the results indicate that there is a metabolic complementation between both bacterial endosymbionts.

Cockroaches are omnivorous insects that harbour Blattabacterium sp. (Flavobacteria, Bacteroidetes). Although the function of these endosymbionts is still unknown, it has been proposed that the blattabacteria might have a beneficial role for the host via an involvement in nitrogen waste recycling. Functional analyses of the genome of Blattabacterium sp. of the German cockroach Blattella germanica provide insights on the extent of the metabolic convergence with Blochmannia (a gamma-proteobacteria), primary endosymbiont of the carpenter ant that also feed on a chemically diverse diet (Gil et al. 2003).

Gil, R., Silva, F. J., Zientz, E., Delmotte, F., González-Candelas, F., Latorre, A., Rausell, C., Kamerbeek, J., Gadau, J., Holldobler, B., van Ham, R. C. H. J., Gorss, R., and Moya, A. (2003) The genome sequence of Blochmannia floridanus: comparative analysis of reduced gemomes. Proceedings of the National Academy of Sciences USA 100:9388-9393. 
Moya, A., Peretó, J., Gil, R., and Latorre, A. (2008) Learning how to live together: genomic insights into prokaryite-animal symbioses. Nature Reviews Genetics 9: 218-229.

Perez-Brocal, V., Gil, R., Ramos, S., Lamelas, A., Postigo, M., Michelena, J. M., Silva, F. J., Moya, A., and Latorre, A. (2006). A small microbial genome: the end of a long symbiotic relationship? Science, 314:312-313.

E-mail: pereto@uv.es

\title{
Never Born Proteins and Never Born Peptidases: Investigation of Peptidase Activity in a Totally Random Library
}

\author{
A. Quintarelli ${ }^{1}$, C. Chiarabelli ${ }^{1,2}$, A. Marcozzi $^{1}$, D. De Lucrezia ${ }^{2,1}$, P. L. Luisi ${ }^{1}$
}

${ }^{1}$ Department of Biology, University of RomaTre, Rome, Italy; ${ }^{2}$ ECLT, European Center for Living Technology, Venice, Italy

The "Never Born Proteins" (NBP) project is based on the concept that the fraction of proteins existing in nature is a minimal part of all theoretical amino acid sequences. An important question is how this fraction of proteins was selected during pre-biotic era. These proteins could have been selected by evolution because they have some particular thermodynamics properties (e.g., thermodynamic or kinetic stability, solubility, etc.); this idea is close to the deterministic point of view supported by de Duve (De Duve, 1995). According to this idea, it is possible to think that the protein existing in nature are the result of the selective pressure, but also the optimal solution to biological necessity. Alternatively, these proteins could be simply the products of contingency, i.e., concomitant accidental environmental conditions that have determined proteins' evolution, in accordance with the theories of other scientists like Monod (Monod, 1971).

All these considerations induced us to look for new polypeptide sequences not selected by Nature but that could have some peculiar characteristics such as catalytic activity.

Our work consisted in producing a library of random proteins, 50 aa long, by phage display. The DNA encoding the Never Born Protein was cloned into a phagemid vector as fusion to gIII, a gene encoding a coat protein, creating a physical linkage between phenotype and genotype. Then the library was selected by bio-panning performing several cycles of selection. The target was a TSA molecule (Transition State Analogue) that mimics the geometric structure of the transition state of a catalytic reaction. According to the state transition theory, during the reaction, reagents pass from a minimal energy state to a transition state with the biggest potential energy where the new bonds are formed partly, then these become stable in final products. A molecule that binds the state of transition can catalyze this reaction. Since TSA in use imitated the geometric structure of a peptide bound hydrolysis, Never Born Proteins positively selected could present peptidase activity.

The selected Never Born Protein were characterized by spectroscopic methods like circular dichroism and their polypeptide sequence was analyzed by Rosetta method to have a structure prediction. Both assays showed the presence of a tertiary stable structure that is an essential prerogative of catalytic activity.

The Never Born Proteins selected in this way are the best candidates to represent prebiotic peptidase and besides they could have an advantageous catalytic activity compared with peptidases selected by the Nature and so they could been called Never Born Peptidase.

This are preliminary results, a starting point for future investigations, more random sequences will be selected, isolated and analyzed to better understand the Never Born Proteins' structures and properties. 
De Duve C. (1995), Vital Dust: life as a cosmic imperative, Ed. Basic Book, New York Monod J. (1971), Chance and Necessity: an essay on the natural philosophy of modern biology, A.A. Knopf, New York

E-mail: aquintarelli@uniroma3.it

\section{Dynamics of Pattern Formation in Biomimetic Systems}

F. Rossi ${ }^{1 *}$, S. Ristori ${ }^{2}$ M. Rustici ${ }^{3}$, N. Marchettini ${ }^{4}$, E. Simoncini ${ }^{4}$, E. Tiezzi ${ }^{4}$

${ }^{1}$ Dipartimento di Chimica Fisica, Università di Palermo, Italy; ${ }^{2}$ Dipartimento di Chimica, Università di Firenze, Italy; ${ }^{3}$ Dipartimento di Chimica, Università di Sassari, Italy; ${ }^{4}$ Dipartimento di Scienze e Tecnologie Chimiche e dei Biosistemi, Università di Siena, Italy

Confinement into restricted spaces is an essential requirement for any process of life and it is thought to have played a mayor role in the emergence of the earliest living systems, by providing concentration of chemical and biological relevant species as well as protection from adverse external environment. In addition to confinement factors, cellular organization involves a complex interaction among structure, chemical kinetics, and transport processes.

By using model systems where these features can be controlled to a large extent independently of the others, the relative contribution of each aspect to cellular attributes can be inferred.

The Belousov-Zhabotinsky (BZ) (Belousov 1958) reaction spontaneously produces complex spatial patterns (spirals, spots,...) that may oscillate in time or remain stationary and for this property it can be considered a valid model for self structuring and self patterning phenomena. Insights gained from the study of the BZ reaction carried out in biomietic matrices may shed light on the emergence of shape in living systems. For example these systems can be used to investigate the occurrence of self-organized patterns in media confined at the nano- to micromicrometer scale, and/or to design a chemical oscillator composed of biological molecules.

In the present work the route followed to develop these ideas was to couple chemical oscillations produced by BZ reaction with confined reaction environments such as direct and reverse micelles (Federico Rossi et al. 2008; Vanag \& Epstein 2008) as model for water pools in a soft matter matrix and phospholipids bilayers (Magnani et al. 2004; Ristori et al. 2007) as model for biological membranes.

Belousov, B.P., 1958. A periodic reaction and its mechanism. In Sbornik Referatov po Radiatsonno Meditsine. Moscow: Medgiz, pagg. 145-147.

Magnani, A. et al., 2004. Chemical waves and pattern formation in the 1,2-dipalmitoyl-snglycero-3-phosphocholine/water lamellar system. Journal of the American Chemical Society, 126(37), 11406-11407.

Ristori, S. et al., 2007. Interplay between the Belousov-Zhabotinsky reaction-diffusion system and biomimetic matrices. Chemical Physics Letters, 436, 175-178.

Rossi, F. et al., 2008. Spatio-Temporal Perturbation of the Dynamics of the Ferroin Catalyzed Belousov-Zhabotinsky Reaction in a Batch Reactor Caused by Sodium Dodecyl Sulfate Micelles. Journal of Physical Chemistry B, 112, 7244-7250.

Vanag, V.K. \& Epstein, I.R., 2008. Patterns of Nanodroplets: The Belousov-ZhabotinskyAerosol OT-Microemulsion System. In Self-Organized Morphology in Nanostructured Materials. Springer Series in Materials Science. Berlin: K. Al-Shamery and J. Parisi, eds., pagg. 89-113.

E-mail: f.rossi@unipa.it 


\title{
Prebiotically Plausible Functional Compartments: A Simulation Model to Study Lipid-Peptide Protocell Dynamics
}

\author{
Kepa Ruiz-Mirazo ${ }^{1,2}$, Marco Lerario ${ }^{3}$, Fabio Mavelli ${ }^{3}$
}

${ }^{1}$ Biophysics Research Unit (UPV/EHU-CSIC), Spain; ${ }^{2}$ Dept. of Logic and Philosophy of Science, University of the Basque Country, Spain; ${ }^{3}$ Dept. of Chemistry, University of Bari, Italy

Simple amphiphilic compounds like fatty acids or isoprenoid derivatives, which have been shown to aggregate spontaneously in aqueous solution forming stable vesicles (Hargreaves \& Deamer, 1978; Pozzi et al. 1996), seem better candidates as protocell components than the more complex phospholipids making up present day biological membranes. In the last years, a number of different experiments have been carried out to gain deeper knowledge on the structural and dynamic properties of this type of prebiotic compartments, as compared to standard liposomes (Chen et al. 2004; Chen \& Szostak, 2004; Cheng \& Luisi, 2003; Rasi et al. 2004; Nomura et al. 2001). The authors recently developed a stochastic simulation platform to study theoretically these systems (Mavelli and Ruiz-Mirazo, 2007a) and have been able to reproduce some of those experimental results (Mavelli and Ruiz-Mirazo, 2007b; forthcoming). However, the challenge for compartmentalists in the field of origins of life remains there: in order to explain why the boundary of the system plays such a fundamental role in the organization of a protometabolic network one has to search not only behind the main structural advantage of semi-permeable membranes (i.e., to maintain relevant concentration thresholds in a local environment), but also behind their functional potential (as the basic scaffolding where transport and transduction mechanisms must be anchored). Actual biomembranes have implemented really sophisticated ways to control the matter and energy flow through the system, but thanks to highly specific protein devices whose appearance is difficult to understand without the long-term action of natural selection. However, given the high prebiotic plausibility of some aminoacids (Miller, 1953), it is quite reasonable to assume that short peptide chains were available from the very beginning. So it is necessary to investigate in which way simple oligopeptides (made of alanine, glycine, serine...) could be incorporated into primitive compartments and change their properties, for sure providing new operational or regulatory capacities to the system. Despite some remarkable attempts to work in this direction (Oliver and Deamer, 1994), little has been done experimentally, so far. Using our simulation model, we will show some results that support this hypothetical 'lipid-peptide' protocell scenario as a worthexploring research avenue (Ruiz-Mirazo \& Mavelli 2008).

Chen I. A. and Szostak J.W., (2004). A Kinetic Study of the Growth of Fatty Acid Vesicles. Biophys. J. 87, 988.

Chen I.A., Roberts R.W. and Szostak J.W., (2004). The Emergence of Competition Between Model Protocells. Science 305, 1474.

Cheng Z. and Luisi P.L., (2003). Coexistence and mutual competition of vesicles with different size distributions. J. Phys. Chem. B 107, 10940.

Hargreaves, WR. Deamer, DW. (1978). Monoalkylliposomes. Biochemistry 17, 3759-3768. Mavelli, F., Lerario, M. and Ruiz-Mirazo, K. (forthcoming) 'ENVIRONMENT': a stochastic simulation platform to study protocell dynamics. BIOCOMP'08 Proceedings.

Mavelli, F. and Ruiz-Mirazo, K. (2007a). Stochastic simulations of minimal selfreproducing cellular systems. Phil. Trans. R. Soc. B 362, 1789.

Mavelli, F. and Ruiz-Mirazo K., (2007b). Stochastic Simulation of fatty acid protocell models. In: Sergey M. Bezrukov, editor, Noise and Fluctuations in Biological, Biophysical, and Biomedical Systems. Bellingham, Washington: SPIE (United States), 6602, 1 B1. 
Miller, S. L. (1953). A production of amino acids under possible primitive Earth conditions. Science 117, 528-529.

Oliver A.E. \& Deamer D.W. (1994). Alpha-helical hydrophobic polypeptides form protonselective channels in lipid bilayers. Biophys J. 66(5): 1364-1379.

Pozzi G., Birault V., Werner B., Dannenmuller O., Nakatani Y., Ourisson G. and Terakawa S., (1996). Single-chain polyprenyl phosphates form "primitive" membranes. Angew. Chem. Int. Ed. Engl., 35: 177-179.

Rasi, S., Mavelli, F. and Luisi, P.L. (2004). Matrix effect in oleate micelles-vesicles transformation. Origins Life Evol. Biosph. 34 (1-2), 215-224.

Ruiz-Mirazo, K. and Mavelli, F. (2008). On the way towards 'basic autonomous agents': stochastic simulations of minimal lipid-peptide cells. BioSystems 91, 374-387.

E-mail: kepa.ruiz-mirazo@ehu.es

\section{Structural Perspective for Comparing Complete Genomes}

Claudia Sierra, Luis Delaye

Microbiology lab, faculty of sciences, UNAM

Now that more than 400 complete genomes from the three domains of life (Archaea, Bacteria and Eukarya) have been sequenced, it is possible to study genomes as phenotypic units and learn about their structure.

A lot of information in this respect has become available, such as $\mathrm{G}+\mathrm{C}, \mathrm{CpG}$ and AT content of the complete genomes. We created a multidimensional method for analyzing this features, all together, with other structural parameters, like the average of DNA internal angles: H, V, L, I (Quintana indexes, 1992), and the distribution of DNA bases according to their physical and chemical characteristics (Index IDH by Cocho and Miramontes, et al, 1995). In this way it was possible to study the structural organization of genomes, and figure out its evolutionary consequences.

We found that the structural organization of DNA in genomes, does not show any important On the other hand, we observed that convergent evolution is predominant in the structural level of genomes. This may suggest that although the range of possibilities in nucleotide organization in the genomes is wide, the multidimensional space in which structural parameters are represented is some how limited for actual forms of life.

Pozzi G., Birault V., Werner B., Dannenmuller O., Nakatani Y., Ourisson G.and Terakawa S., (1996). Single-chain polyprenyl phosphates form "primitive" membranes. Angew. Chem. Int. Ed. Engl., 35: 177-179.

E-mail: mesiclau_74

\section{Rooting the Universal Tree of Life}

Ryan G. Skophammer ${ }^{1}$, Craig W. Herbold ${ }^{2}$, Jacqueline A. Servin ${ }^{2}$, James A. Lake ${ }^{1,2,3}$

${ }^{1}$ Dept. of Molecular Cell and Developmental Biology, UCLA; ${ }^{2}$ Molecular Biology Interdepartmental Program, UCLA; ${ }^{3}$ Dept. of Human Genetics, UCLA

Determining which extant organisms are most closely related to the cenancestral population allows inferences to be made regarding the origin of life and the emergence of major biological 
metabolic innovations. To this end, we have designed an algorithm to eliminate the root of the universal of tree of life from major taxa: top-down rooting. Conserved protein sequences are aligned with paralogous outgroups and the pattern of indel presence and absence is recorded for each group. If an indel is present, the group is given the state "+"; if it is absent, the group is given the state "-"; if the protein is missing from a group, the group is given the state "m". Parsimony is applied to the character state changes to determine which trees are least parsimonious. Eliminating these trees allow us to eliminate possible rooted universal trees. Using this algorithm, we have found evidence eliminating the root from the double-membrane prokaryotes, the actinobacteria, and a clade consisting of the firmicutes and Archaea. These findings imply that the cenancestral population was likely mesophilic, gram-positive, surrounded by a peptidoglycan layer, and enclosed by ester-linked lipids.

Lake JA, Herbold CW, Rivera MC, Servin JA, Skophammer RG. (2007). Rooting the tree of life using nonubiquitous genes. Molecular Biology and Evolution, 24:130-136.

Servin JA, Herbold CW, Skophammer RG, Lake JA. (2008). Evidence excluding the root of the tree of life from the actinobacteria. Molecular Biology and Evolution, 25:1-4.

Skophammer RG, Herbold CW, Rivera MC, Servin JA, Lake JA. (2006). Evidence that the root of the tree of life is not within the Archaea. Molecular Biology and Evolution, 23:1648-1651. Skophammer RG, Servin JA, Herbold CW, Lake JA. (2007). Evidence for a gram-positive, eubacterial root of the tree of life. Molecular Biology and Evolution, 24:1761-1768.

E-mail:skop@ucla.edu

\title{
Proterozoic Stromatolites and Microfossils from the Lesser Himalaya, India: Unicellular to Multicellular Evolution of Life
}

\author{
Vinod C. Tewari
}

Wadia Institute of Himalayan Geology, Dehradun, Uttarakhand, India and A.S.International Centre for Theoretica Physics, Trieste, Italy

The Meso-Neoproterozoic and Terminal Proterozoic succession of the Lesser Himalaya in the northern India shows excellent preservation of stromatolites and microorganisms from the Jammu Limestone in the NW and Buxa Dolomite in the NE. The most dominant stromatolite assemblage include Colonnella columnaris, Kussiella kussiensis, Conophyton cylindricus, C. garganicus, Jacutophyton, Baicalia, Jurusania, Gymnosolen, Minjaria, Inzeria, Tungussia, Boxonia and Stratifera. The Krol belt in the central Lesser Himalaya is characterized by mostly stratified and small conical and columnar forms like Stratifera, Conistratifera, Conophyton, Aldania and Collumnaefacta.(Tewari, 1989, 1993, 2004, 2007). Deoban and Buxa black cherts show highly diversified permineralised microbiota. Cyanobacteria found in the Deoban and Buxa cherts include Huronispora psilata, Myxococcoides minor, Glenobotrydion aenigmatis, Siphonophycus, Oscillatoriopsis, Obruchevella, and Kildinosphaera (Tewari, 2004, Shukla et al 2006, Schopf et al. 2008). The acritarchs show morphological changes through time and therefore has been used as stratigraphic marker in the Infra KrolKrol cherts of the Lesser Himalaya. The acanthomorphic acritarchs and leiosphaerids are present in the Infra Krol cherts and disappear before the emergence of the Ediacaran biota in the Krol Formation. The acanthomorphs in the Infra Krol and Buxa cherts include Micrhystridium, Trachysphaeridium and Vandalosphaeridium. The multicellular red brown algae Vendotaenia, Krolotaenia, Tyrasotaenia, have been recorded from the Lower Krol Formation (Tewari, 1989, 2004). The Ediacaran assemblage has been recorded 
The Upper Krol Formation of the Lesser Himalaya. The assemblage include the soft bodied metazoans Cyclomedusa davidi, Charniodiscus sp., fronds and disc, Kimberella cf, quadrata, Zolotytsia biserialis and Conomedusites lobatus (Tewari, 2004, 2007).

The Terminal Proterozoic diversification of life that led to the radiation of animal and plants occurred between 0.59 and 0.53 billion years ago on earth. The prokaryotic to eukartotic evolution and diversification of life, palaeoclimatic event of Neoproterozoic snowball earth and the extinction and reemergence of highly evolved life after Blainian/ Marinoan glaciation is well preserved in the Lesser Himalaya of India.

Schopf, J.W., Tewari, V.C., and Kudravtsev, A. (in press). Discovery of a new chert permineralised microbiota in the Proterozoic Buxa Formation of the Ranjit window, Sikkim, NE India, and its Astrobiological implications. To appaear in the Astrobiology. Shukla, M., Tewari, V.C., Babu, R.and Sharma, A. (2006) Microfossils from the Neoproterozoic Buxa Dolomite West Siang district, Arunachal Lesser Himalaya, India and their significance. Jour. Palaeont. Soc. India, 51: 57-73.

Tewari, V.C. (1989) Upper Proterozoic-Lower Cambrian stromatolites and Indian stratigraphy. Him. Geol. 13: 143-180.

Tewari, V.C. (1993) Precambrian and Lower Cambrian stromatolites of the Lesser Himalaya, India. Geophytology, 23: 19-39.

Tewari, V.C. (2004) Microbial diversity in Meso-Neoproterozoic formations, with particular reference to the Himalaya. In Seckbach, J., editor, Origins, pages 515-528. Kluwer Academic Publishers, The Netherlands.

Tewari, V.C. (2007) The rise and decline of the Ediacaran biota: palaeobiological and stable isotopic evidence from the NW and NE Lesser Himalaya, India. In Vickers Rich, P and Komarower, P. editors, The Rise and Fall of the Ediacaran biota. pages 77-102. The Geological Society of London.

E-mail: vtewari@wihg.res.in

\section{Photonics of Folate Coenzymes in Relation to Evolution}

Yuliya L. Vechtomova, Taisiya A. Telegina, Mikhail S. Kritsky

\section{A.N. Bach Institute of Biochemistry, Russian Academy of Sciences, Moscow, Russia}

The important role of pteridines (pterins, folates) as coenzymes for key reactions of cell metabolism along with availability of pteridines under conditions mimicking prebiotic world (Heinz et al., 1979), suggests their plausible participation in metabolism of protobionts. Pteridines as well as benzopteridines (flavins) are photoreactive molecules, which sensitize electron and energy transfer reactions induced by UVA. We believe that excited pteridines which can oxidize electron donors with a highly positive redox potential and drive the uphill electron transfer played role in primitive metabolism as photocatalysts and participants of solar energy conservation processes (Kritsky and Telegina, 2004).

Some pteridine coenzymes, when excited, demonstrate chemical activity similar to that of pteridine coenzymes bound to specific apoenzyme. Nevertheless, photoexcitation could not totally compensate the absence of genetically ordered and functionally specific apoproteins in primitive metabolism. For instance, some monocarbon derivatives of tetrahydrofolate lack photochemical activity what means that photocatalytic processes were not sufficient to ensure H4-folate-mediated metabolism of monocarbon fragments 
such as methyl and formyl groups. At the same time, low photochemical activity and stability of 5,10-methenyltetrahydrofolic acid (MTHF) against photochemical oxidation is a prerequisite for non-radiative energy transfer from this excited molecule and may have favored a selection of this molecule for light-harvesting antenna in photoreceptor proteins DNA-photolyase and cryptochrome (Sancar, 2003). The other properties essential for selection of MTHF for antenna pigment were high photon absorptivity (the $\varepsilon_{\max }=$ $\left.26,000 \mathrm{M}^{-1}\right)$ and the long-wave shifted absorption maximum $\left(\lambda_{\max }=360 \mathrm{~nm}\right)$ as compared to other H4-folates. The combination of these properties in MTHF results from the presence in its molecule of imidazoline ring adjacent to pteridine heterocycle and the protonated state of tetrahydropteridine cycle (Telegina et al., 2005). Interestingly, MTHF was conserved as antenna pigment in light-sensitive proteins of eukaryotic organisms whose evolution proceeded in oxygen-rich atmosphere. At the same time, in some prokaryotes including archea and cyanobacteria, another compound, 7,8-didemethyl-8-hydroxy-5-deazariboflavin plays this role in DNA photolyases (Sancar, 2003). Unlike deazaflavin, found only in few microbial species, MTHF is a participant of cell metabolism in a variety of pro- and eukaryotic organisms.

Supported by Program of Basic Research No 18 of Russian Academy of Sciences and by grants NoNo 07-04-00460_a and 06-04-90599-BNTS_a from Russian Foundation for Basic Research.

Heinz, B., Ried, W., Dose, K. (1979). Thermische Erzeugung von Pteridinen und Flavinen aus Aminosaueregemischen. Angewandte Chemie, 91(6):510-511

Kritsky, M.S. and Telegina, T.A. (2004). Role of nucleotide-like coenzymes in primitive evolution. In Seckbach J., editor, Origins Genesis, Evolution and Diversity of Life, pages 215-231. Kluwer, Dordrecht.

Sancar, A. (2003). Structure and function of DNA photolyase and cryptochrome blue-light photoreceptors. Chemical Reviews. 103:2203-2237

Telegina, T. A., Lyudnikova, T. A., Zemskova, Yu. L., Sviridov, E. A., and Kritsky, M. S. (2005). Resistance of 5,10-methenyltetrahydrofolate to ultraviolet radiation. Applied Biochemistry and Microbiology. 41(3):275-282

E-mail: vechtomova@inbi.ras.ru

\section{Low Complexity in Regions in Lentiviral Proteins}

Ana Maria Velasco, Luis Delaye, Arturo Becerra, Antonio Lazcano

Facultad de Ciencias, UNAM, Apdo. Postal 70-407, Ciudad Universitaria, Mexico D. F. 04510, MEXICO

The presence of low complexity regions (LCR) has been confirmed in sequences of the three cellular linages (Bacteria, Archaea and Eucarya). Nevertheless, the role that they play is not yet fully understood. Much less is know about viral LCRs. In this work we present the results of the analysis of the products of the genes of each the major groups of lentivirus using the completely sequenced genomes of lentivirus available in public databases. We found that a significant fraction of them displays a LCR at least. The highest number of LCR was found in the polypeptidic product of the env gene, while in gag and pol there are three and two LCR respectively. It is important to note that in the accesory genes which are characteristic of this group of retrovirus, one or two zone present LCR. These results will be discussed.

E-mail: ana.velasco@servidor.unam.mx 


\section{A Synthetic Protocell Model with a Self-Encoded System}

Tetsuya Yomo ${ }^{1,2}$

${ }^{1}$ Department of Bioinformatics Engineering, Graduate School of Information Science and Technology, Osaka University, Japan; ${ }^{2}$ Exploratory Research for Advanced Technology (ERATO), Japan Science and Technology Agency (JST)

In all living systems, the genome is replicated by proteins encoded within the genome itself, which is an essential reaction for the sustentation and evolution in biological systems. To mimic such universal process, we constructed a simplified system comprised of a minimal set of biological components in which the genetic information is replicated by a selfencoded replicase. In this system, designated as the RNA-protein self-replication system, the catalytic subunit of replicase is synthesized from the template RNA that encodes itself, the replicase subsequently replicates the template RNA used for its own production.

This synthetic self-replicating system is one of the simplest systems available, consisting of just 144 gene products, which is comparable to the hypothetical minimal cell with approximately 150 gene products. It was further encapsulated within a microcompartment bounded by a lipid bilayer, so called liposome, resulting in a compartmentalized selfreplicating system. The information and the function for its replication are encoded on different molecules and are compartmentalized into the microenvironment for evolvability. Successful construction of this in liposome self-replicating system shows a significant step toward synthetic life, as well as provides a further insight to the protomodel of cellular life.

Luisi, P. L., Ferri, F. and Stano, P. (2006) Approaches to semi-synthetic minimal cells: a review. Naturwissenschaften 93, 1-13.

Shimizu, Y. et al. (2001) Cell-free translation reconstituted with purified components. Nat. Biotechnol. 19, 751-755.

Sunami, T. et al. (2006) Femtoliter compartment in liposomes for in vitro selection of proteins. Anal. Biochem. 357, 128-136.

Szostak, J. W., Bartel, D. P. and Luisi, P. L. (2001) Synthesizing life. Nature 409, 387-390.

E-mail: yomo@ist.osaka-u.ac.jp

\section{A Novel Evolution Concept of Protein Phosphorylation Mechanism}

Zeng Zhiping, Liu Linna, Zhao Yufen

Department of Chemistry, the Key Laboratory for Chemical Biology of Fujian Province, College of Chemistry and Chemical Engineering, Xiamen University, Xiamen 361005, People's Republic of China

Protein phosphorylation controls many cellular processes, but its mechanism is still a debatable subject, especially for protein kinases. According to the chemical property of $\mathrm{N}$-phosphoamino acids, we deduce a novel three-step covalent mechanism (Ni et al., 2005), which is much different from 'in-line phosphorus transfer' mechanism (Valief et al., 2003).

It is known that human contains 518 kinds of protein kinases to regulate the cell's signal. Among them, more than $80 \%$ are the serine, threonine and tyrosine kinases with the hydroxyl group as the receptors phosphotransferases with a alcohol group as acceptor (E.C 
2.7.1.X).While in the literature, there are phosphotransferases with a nitrogenous group as acceptor (E.C 2.7.3.X) and phosphotransferases with a carboxyl group as acceptor (E.C 2.7.2.X). Therefore, it might be a reasonable approach to illuminate the kinases catalyzing the phosphoryl transfer mechanism by comparison of these three types of kinases.

These three types of kinases, catalyze the $\gamma$-P of the ATP transfer to their corresponding substrates with three different phosphoryl groups of receptors, namely the HO-receptor, $\mathrm{H}_{2} \mathrm{~N}$-receptor and the HOOC-receptor (see figure 1).

By the thermodynamical data, it seems that the carboxyl mixed anhydride $\mathbf{1}$, easy to hydrolysis, contain much higher energy than the phosphoamide bond $2\left(617 \mathrm{~kJ} \mathrm{~mol}^{-1}\right)$, which in turn is higher than the phosphoester bond $3\left(597 \mathrm{~kJ} \mathrm{~mol}^{-1}\right)$ (Lange).

In this paper, by the evolution investigation, the Ser/Thr kinases phosphoryl transfer mechanism might go through the combination of the P-NH-residues and the P-OOCresidues mechanism. since the key catalytic residues of Ser/Thr kinases are Lys and Asp, it was proposed that the $\gamma$-P of the ATP is not directly transfer to the substrate, but might be proceeded by $\gamma$-P-Lys and $\gamma$-P-Asp high-energy intermediates and then finally phosphorylate the substrate.

\section{E.C 2.7.2.X (phosphotransferases with a carboxyl group as acceptor)}

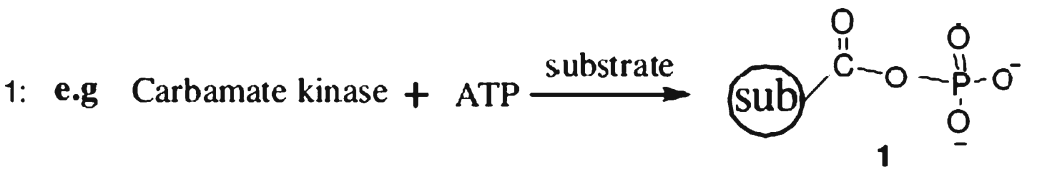

E.C 2.7.3.X (phosphotransferases with a nitrogenous group as acceptor)

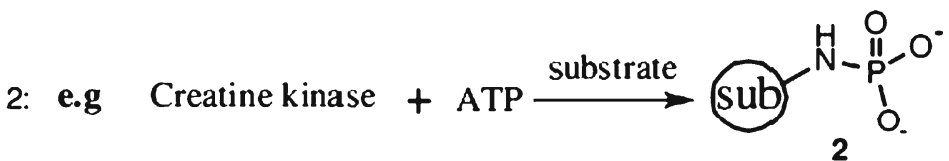

E.C 2.7.1.X (phosphotransferases with a alcohol group as acceptor).

3: e.g Serine kinase $+\operatorname{ATP} \stackrel{\text { substrate }}{\longrightarrow}$ sub

3

\section{figure 1}

Lange's Chemistry Handbook Version 15th. section 4. properties of atoms, radicals, and bonds.

Ni, F., et al., Analysis of the phosphoryl transfer mechanism of c-AMP dependent protein kinase (PKA) by penta-coodinate phosphoric transition state theory. Current Protein \& Peptide Science, 2005. 6(5): p. 437-442.

Valiev, M., et al., The Role of the Putative Catalytic Base in the Phosphoryl Transfer Reaction in a Protein Kinase: First-Principles Calculations. Journal of the American Chemical Society, 2003. 125(33): p. 9926-9927.

E-mail: zengzhiping@xmu.edu.cn 


\section{Precellular Evolution}

\section{A Trade-Off Between Neutrality and Adaptability Limits the Optimization of Viral Quasispecies}

Jacobo Aguirre

Centro de Astrobiología, INTA-CSIC. Ctra. de Ajalvir km. 4, 28850 Torrejón de Ardoz, Madrid, SPAIN

Theoretical studies of quasispecies, concept presented in (Eigen, 1971), usually focus on two properties of those populations at the mutation-selection equilibrium, namely asymptotic growth rate and population diversity. Recently, it has been postulated that quasispecies endowed with a high degree of neutrality perform better in competition experiments, since neutrality improves the capacity of a population to withstand the deleterious effect that most mutations have on fitness. In this study we analyse how the optimization of equilibrium properties is affected when a quasispecies evolves in an environment perturbed through frequent bottleneck events (Aguirre, et al. 2008). By means of a simple model we demonstrate that high neutrality may be detrimental when the population has to overcome repeated reductions in the population size, and that the property to be optimized in this situation is the time required to regenerate the quasispecies, i.e. its adaptability. In the scenario described, neutrality and adaptability cannot be simultaneously optimized. When fitness is equated with long-term survivability, high neutrality is the appropriate strategy in constant environments, while populations evolving in fluctuating environments are fitter when their neutrality is low, such that they can respond faster to perturbations. Our results might be relevant to better comprehend how a minoritary virus could displace the circulating quasispecies, a fact observed in natural infections and essential in viral evolution (de la Torre and Holland, 1990; Aguirre and Manrubia, 2007).

Aguirre, J., Manrubia, S. C., and Lázaro, E. (2008). A trade-off between neutrality and adaptability limits the optimization of viral quasispecies (preprint).

Aguirre, J. and Manrubia, S. C. (2007). Out-of-equilibrium competitive dynamics of quasispecies. Europhys. Lett. 77:38001.

Eigen, M. (1971). Selforganization of matter and the evolution of biological macromolecules. Naturwissenschaften 58:465-523.

de la Torre, J. C. and Holland, J. J. (1990). RNA virus quasispecies populations can suppress vastly superior mutant progeny. J. Virol. 64: 6278-6281.

E-mail: aguirreaj@inta.es

\section{Molecular Evolution in the Primitive Earth: Nonlinear Analysis of Archaea tRNAs Compared to Computer-Generated Random Sequences}

G. Bianciardi ${ }^{1}$, L. Borruso ${ }^{2}$

${ }^{1}$ Dipartimento di Patologia Umana e Oncologia, Università di Siena, Via delle Scotte 6, 53100 Siena, Italia/ Centro Studi di Esobiologia, Milano, Italy; ${ }^{2}$ Dipartimento di Scienze e Tecnologie Alimentari Microbiologiche (DISTAM), Università degli Studi di Milano, Italia

Nothing is known about the way(s) from which life born, and plausibile pathways of prebiotic evolution remain obscure, however, in that context, RNA may be considered the 
most oldest known informational genetic polymer (Howland, 200). Billions years ago, according to the exon theory of genes (Di Giulio, 1998), small RNAs translated into peptides of 15-20 aminoacids: minigenes of pre-tRNAs codifying RNA hairpin structures. The dimerization of two equal RNA hairpin structures may have lead to the formation of the cruciform structure of the tRNA molecule: tRNAs may reflect the primordial genes of that era.

Nucleotide sequence data of tRNAs in archaea were obtained from the GeneBank library*. Random sequence data (white noise) were obtained from the algorithm by Press and Teukolsky (1992). Nucleotide sequences were analyzed as random walks, where each base represent a different step in a two-dimensional space; vice versa, the uniform and random distributed data points over the unit interval algorithm-generated were divided in 16 intervals to which A,C,G,T (U), letters were attributed.

Nonlinear parameters (relative LZ complexity, largest Lyapunov exponent, Hurst exponent, correlation dimension, entropy, BDS statistic, Manhattan and Euclidean fractal dimensions) of nucleotide sequences and computer-generated random sequences were evaluated making use of Chaos Data Analyzer (Sprott \& Rowlands (1995) or Gates' (1986) formulation (fractal dimensions).

Our data show that the values of nonlinear parameters obtained from the archaea are lower than the values of randomly generated sequences $(p<0.01)$.

These data are in agreement with the ones by Weiss et al. (2000), showing a significant reduction of the Shannon entropy $(-1 \%)$ in protein sequences compared to random polypeptides. Our results suggest that in the primitive Earth informational polymers might be originated from slightly edited random strings and that during biologic evolution the distance from pure randomness increased. Deviation from pure randomness should be arisen from some constraints like the secondary structure of the biologic macromolecules.

Di Giulio M., Reflections of the Genetic Code: a Hypothesis. J. Theor. Biol., 191, 2, 191196, 1998.

Gates M.A., A simple way to look at DNA, J. Theor. Biol., 119, 319-328, 1986.

Howland J.L., The Surprising Archaea, Oxford University Press, 2000.

Press W.H. \& Teukolsky S.A., Portable Random Number Generators, Computers in Physics, 6, 522-524, 1992.

Sprott J.C. \& Rowlands G., Chaos data Analyzer, Physics Academic Software, 1995.

Weiis O. et al., Information Content of Protein Sequences, J. Theor. Biol., 206, 379-386, 2000.

* http://www.ncbi.nlm.nih.gov/

E-mail: gbianciardi@unisi.it

\section{Evading Quantum De-coherence in Living Matter by Feshbach Resonance}

Antonio Bianconi, Rocchina Caivano, Nicola Poccia, Alessandro Ricci, Alessandro Puri, Michela Fratini

Department of Physics, La Sapienza University of Rome, 00185 Roma, Italy

In these last years the genomes of many species have been sequenced, and the structures of many macromolecular machineries of the cell have been solved by synchrotron radiation. The new challenge of the post-genomic era is to study how molecular machineries actually work together in the space-time inside the living cells. 
The consensus is growing that the emergence of the living cell from prebiotic syntheses is related with the onset of a particular phase of matter made of a macroscopic coherent state of biochemical reactions where the interaction with the ambient results in the Darwinian evolution. The coherent state of living matter could emerge in the proximity of a critical point (biological order at the edge of caos) (Rupley et al. 1988), and there is growing experimental evidence that quantum coherence could play a relevant role (Engel et al., 2007, Hagan et al. 2002). In this scenario the key physical problem is how it is possible that the quantum coherence phase could resist to the de-coherence attacks of temperature (Barrow et al. 2004; Davies 2004). The superfluid phase has been taken as the simple physical model system for macroscopic quantum coherence (Coleman, 2007).

We show that by selecting particular nanoscale architectures and driving the system close a to a quantum critical point it is possible to realize a particular superfluid that is able to avoid temperature de-coherence effects. We show that a particular quantum critical point can be reached at a critical values of (a) density, (b) disorder, (c) chemical pressure and (d) temperature (Fratini et al 2008) where the quantum many body Feshbach resonance or shape resonance (Bianconi 2005 and 2007, Bianconi et al. 2007) for molecular association and dissociation processes is actually effective to give a macroscopic quantum coherent phase that avoids the temperature quantum de-coherence effects. We show that the proximity to a particular quantum critical point is related with the emergence of the Feshbach resonance. We discuss this scenario for the case of biochemical reactions in the thylakoid membrane.

Barrow J. D., Davies P. C. W. Davies. Harper, Jr C. L. 2004 "Science and Ultimate Reality Quantum Theory, Cosmology, and Complexity" Cambridge University Press.

Bianconi A., 2005 "Feshbach shape resonance in multiband superconductivity in heterostructures" Journal of Superconductivity 18, 25; and Bianconi Antonio 2007. "Feshbach shape resonance for high Tc superconductivity in superlattices of nanotubes" arXiv:0712.0061

Bianconi A. and Vittorini-Orgeas A. "From the Majorana Theory of Incomplete P' Triplet to Feshbach Shape Resonances" Proceeding of the Meeting Ettore Majorana's legacy and the Physics of the XXI century (University of Catania, Italy 5-6 October, 2006) Published on line in Proceedings of Science POS(EMC2006)-001

Coleman, P. 2007 "Frontier at your fingertips", Nature 446, 379.

Davies, P. C. W. 2004 “Quantum fluctuations and life”, arXiv:quant-ph/0403017.

Engel G. S., Calhoun T. R., Read E. L., Ahn T-K, Mancal T., Cheng Y-C. Blankenship R. E. \& Fleming G. R. 2007 "Evidence for wavelike energy transfer through quantum coherence in photosynthetic systems" Nature 446, 782.

Fratini M, Poccia N, and Bianconi A 2008 "The Feshbach resonance and nanoscale phase separation in a polaron liquid near the quantum critical point for a polaron Wigner crystal" Journal of Physics: Conference Series 108, 012036.

Hagan S., Hameroff S. R., and Tuszyn J. A., 2002 "Quantum computation in brain microtubules: Decoherence and biological feasibility” Phys. Rev. E. 65, 061901.

Rupley J.A., Siemankowski L., Careri G. and Bruni F. 1988 "Two-dimensional protonic percolation on lightly hydrated purple membrane" Proc Natl Acad Sci U S A. 85: 9022

E-mail: Antonio.Bianconi@roma1.infn.it 


\title{
Bottlenecked Populations of Naked RNA Genes Can Circumvent Muller's Ratchet
}

\author{
Carolina Diaz ${ }^{1}$, Niles Lehman ${ }^{2}$ \\ ${ }^{1,2}$ Portland State University, Portland, Oregon, USA
}

Preservation of the genetic information over time is relevant to the survival of populations. At the origins of life, asexual populations of short naked RNA-genes must have been more susceptible to the detrimental effect of mutation accumulation via Muller's ratchet. It has been well demonstrated experimentally that abiotic asexual bottleneck populations are in fact susceptible to become extinct in consequence of the synergistic effect of Muller's ratchet and random drift. Using an in vitro continuous evolution model asexual bottlenecked ligase ribozyme populations of 100, 300, 600, and 3,000 molecules are allowed to replicate at various mutational rates. The average time to extinction due to the accumulation of mutations was found inversely related with the effective population size (Soll et al., 2007). Higher mutational rates generate a broader array of mutations as expected, including not only deleterious mutations but also beneficial mutations. A highly recurrent beneficial mutation has been observed to completely displace the wild type in some lineages, while in others is in strong competition with it. The population jumps back and forth between two fitness peaks of the landscape. Sexual reproduction introduced in small lineages allows them to circumvent Muller's ratchet via recombination, an available solution for small populations of naked genes to achieve larger population sizes at the origins of life.

Soll, S.J., Arenas, C.D., Lehman, N. (2007). Accumulation of deleterious mutations in small abiotic populations of RNA. Genetics, 175:267-275.

E-mail: cdiaz@pdx.edu

\section{Photosynergistic Collaboration of Non-linear Processes at Mesoscopic Level in a Irradiated Sterilized Aqueous Mixture of Some Inorganic and Organic Substances and Formation of Functionally Integrated Self-Sustaining Supramolecular Assemblies, "JEEWANU"}

\section{V.K. Gupta}

Laboratory of Molecular Evolution, Department of Zoology, C.M.D. Post Graduate College, Bilaspur-495 001 (Chattisgargh), India

Irradiated sterilized aqueous mixture of some inorganic and organic substances shows the photochemical formation of open chain energy transducing protocell-like molecular associations. They multiply by budding, grow from within and show various metabolic activites in them (Bahadur, and Ranganyaki,1970) The various microscopic investigations using optical microscope, SCM, TEM and AFM have revealed that they have a definite boundary wall and intricate internal structure. They have been analysed to contain a number of biochemical-like substances in them. The ultra fast laser flash photolysis $\left(10^{-9}\right.$ to $10^{-20} \mathrm{~ns}$ ) also showed the formation of photoproducts in the mixture. In prebiotic atmosphere possibly photosynergistic collaboration of non-linear processes at mesocopic level led to the and formation of structurally organized and functionally integrated, self- 
sustaining, photoautotrophic supramolecular assemblies, similar to "Jeewanu" which might have catalysed energy transduction in the primitive atmosphere.

E-mail: guptavin1@rediffmail.com

\section{Quantum Mechanics and the Emergence of Life Giving Catalysts}

Nathan Haydon ${ }^{1,3}$, Shawn McGlynn ${ }^{1,2,3}$, Olin Robus ${ }^{1,3}$, Prasanta Bandyopadhyay ${ }^{1,3}$, Gordon Brittan ${ }^{1,3}$

${ }^{1}$ NASA Astrobiology institute; Astrobiology Biogeocatalysis Research Center; ${ }^{2}$ Department of Chemistry and Biochemistry; ${ }^{3}$ Department of History and Philosophy, Montana State University Bozeman, MT 59717

Quantum mechanics, as the most successful theory to date to describe the physical world, plays an important role in all physical processes including those associated with living matter. Recently, attempts have been made by several authors to explore the role and effects of quantum phenomenon on biological processes and structures. Here we analyze these attempts, highlighting key concepts and problems which have yet to be addressed. Continuing from this, we present several examples which we believe to be more prevalent and more accurate representations of the effects of quantum mechanics on life, and in particular, the origins of life. In the context of an iron sulfur dominated mound as espoused by Russell and others, we suggest that quantum mechanics may have played a role in the origin of efficient catalysts that eventually led to biological complexity. In particular, within iron sulfur compartments quantum decoherence allows for rapid exploration of possible catalysts and assists in giving rise to those capable of supporting reactions that lead to the proliferation of biologically favorable molecules.

E-mail: njhaydon@gmail.com

\section{Characteristics of Fluctuating Conditions in the Hydrothermal Medium Suitable for the Origin of Life}

\section{Kompanichenko ${ }^{1}$, Pol. Kralj ${ }^{2}$, Pet. Kralj ${ }^{3}$, E. Frisman ${ }^{1}$}

${ }^{1}$ Institute for Complex Analysis, Birobidzhan, Russia; ${ }^{2}$ Geological Survey of Slovenia, Ljubljana, Slovenia; ${ }^{3}$ Gejzir, EON Research Centre, Ljubljana, Slovenia

In accordance with the proposed systemic conception of the origin of life, the transition of prebiotic microsystems into simplest living units might occur only under oscillating thermodynamic and physic-chemical parameters (Kompanichenko, 2008). The significant oscillations are peculiar to hydrothermal systems including their outcrops in ocean and especially terrestrial groundwater aquifers. The scale of the oscillations depends on the tectonic-magmatic and seismic activity of a geothermal region. Exploration of thermodynamic and physico-chemical fluctuations in natural hydrothermal fields can be helpful to base laboratory experiments on prebiotic chemistry under changeable conditions that gives us a chance to approach to experimental obtaining of a really living unit.

To characterize a scale of the thermodynamic and physic-chemical fluctuations four hydrothermal fields were explored. Two of them (Mutnovsky and Pauzhetsky hot springs) represent active volcanic region-Kamchatka peninsula in eastern Russia. The rest Mura 
(Slovenia) and Kuldur (Russian Far East) geothermal fields are situated in volcanically nonactive regions. Temperature of water and water-steam mixture in wells of Mutnovsky and Pauzhetsky fields ranges from less than $100^{\circ} \mathrm{C}$ up to $240^{\circ} \mathrm{C}$, water in Mura and Kuldur thermal basins is characterized with the temperature $50-70^{\circ} \mathrm{C}$. Data of monitoring of pressure, temperature and some chemical parameters in wells of these fields were mathematically processed. Periods of long-range macrofluctuations of pressure and temperature in Mutnovsky and Kuldur fields are 2-4.5 months, maximum amplitudes of temperature on orifices of the wells are $53^{\circ} \mathrm{C}$ and $9{ }^{\circ} \mathrm{C}$ correspondingly, and maximum amplitude of pressure in Mutnovsky field is 34 bars. Periods of short-range minioscillations are 10-70 min in Mutnovsky, Pauzhetsky and Mura fields, and average amplitudes of pressure are $0.2-0.7$ bars. Amplitudes of minioscillations of temperature and $\mathrm{pH}$ in Mura basin are $1-2^{\circ} \mathrm{C}$ and 0.2 correspondingly (Kralj, 2000). There exists strict positive correlation of temperature with $\mathrm{pH}, \mathrm{K}^{+}, \mathrm{Na}^{+}, \mathrm{Ca}^{2+}, \mathrm{HCO}_{3}{ }^{-}, \mathrm{SO}_{4}{ }^{2-}, \mathrm{Cl}^{-}, \mathrm{F}^{-}$, concentrations of $\mathrm{Mg}^{2+}, \mathrm{NH}_{4}{ }^{+}, \mathrm{CO}_{2}$ change independently. The general conclusion is that minioscillations of thermodynamic and physico-chemical parameters in hydrothermal systems are usual phenomenon. From time to time the parameters significantly change because of macrofluctuations that can be initiated by various causes (including earthquakes and volcanic eruptions). Such changeable nonequilibrium medium is suitable to be considered as potential geological Cradle of life on the early Earth.

Kompanichenko, V.N., 2008. Three stages of the origin-of-life process: bifurcation, stabilization and inversion. International Journal of Astrobiology, Volume 7, Issue 01, p. 27-46.

Kralj, Pt., Kralj, Pol., 2000. Thermal and mineral waters in north-eastern Slovenia. Environmental Geology 39 (5), 488-498.

E-mail: kompanv@yandex.ru

\section{Organic Matter in Hydrothermal Systems of Kamchatka: Relevance to the Origin of Life}

Kompanichenko V.N.

Institute for Complex Analysis, Birobidzhan, Russia

Fluctuating thermodynamic and physico-chemical parameters were likely to play a role in the origin of life by concentrating organic reactants and driving covalent bond formation (Kompanichenko, 2008). In order to provide insight about the kinds of organic compounds that were likely to be available in fluctuating geothermal environments on the early Earth, I have investigated the chemical composition of hydrothermal systems in the Kamchatka peninsula and adjoining regions of eastern Russia. Samples were taken from hot springs far from potential sources of contamination by human populations, and from boreholes 16 to $1,200 \mathrm{~m}$ in depth. The temperature ranged from $175^{\circ} \mathrm{C}$ (sterile water-steam mixture) to $55^{\circ} \mathrm{C}$ (hot water with thermophile populations). The samples were analyzed by gas chromatography-mass-spectrometry (Shimatsu GCMS-QP20105). Phthalates were present in all samples, presumably as trace contaminants from plastic containers. The highesttemperature $\left(175^{\circ} \mathrm{C}\right)$ sample from a borehole contained only polycyclic aromatic hydrocarbons (naphthalene, biphenyl, phenathrene, fluorene, 1-methylnaphtaline). These organic compounds characterize the deep sterile zone near the active Mutnovsky volcano (depth 200-600 m, temperature $175-250^{\circ} \mathrm{C}$ ). Biphenyl and phenathrene were absent in samples from lower temperature boreholes $\left(95-124^{\circ} \mathrm{C}\right)$ and springs. However, numerous other 
aromatic hydrocarbons (benzenes, xylenes) and aliphatic hydrocarbons (decanes, isoalkanes) were present.

The source of these compounds is not yet established. They may represent pre-existing organic material that has been chemically degraded by pyrolysis. For instance, Simoneit et al. (2008) established that the light oil associated with the Uzon caldera in Kamchatka was formed by pyrolysis of buried algal mats. More interesting would be to determine that the aromatics and alkanes are products of a Fischer-Tropsch type synthesis. However, the original source of organics was not so important for the origin of life on the early Earth: these compounds might as to be synthesized in hydrothermal medium as to be involved into hydrothermal circulation from other sources (synthesis at pre-geological stage of the Earth formation, synthesis in the atmosphere/ocean at the expense of ultraviolet radiation, delivering by comets, etc.). It seems that organic matter of any origin had a chance to be transformed into a living unit under oscillating hydrothermal conditions through three successive stages: (1) an organic microsystem becomes unstable at the critical point of the bifurcate transition under conditions far from equilibrium; (2) relative stabilization of the microsystem due to the balanced oscillations around the critical point (appearance of the paradoxical state "stabilized instability"); (3) inversion of the energetic balance-free energy contribution begins to prevail over entropy contribution (Kompanichenko, 2008).

Kompanichenko V.N. Three stages of the origin-of-life process: bifurcation, stabilization and inversion // International Journal of Astrobiology, 2008, Volume 7, Issue 01, p. 27-46. Simoneit, B., Deamer D.W. and Kompanichenko, V. 2008. Characterization of hydrothermally generated oil from the Uzon Caldera, Kamchatka. Applied Geochemistry (In press).

E-mail: kompanv@yandex.ru

\section{Specialization of Early Replicators in the Metabolic Replicator Model System}

\section{Balázs Könnyü ${ }^{1}$, Tamás Czárán ${ }^{2}$}

${ }^{1}$ Eötvös University, Department of Plant Taxonomy and Ecology; ${ }^{2}$ Hungarian Academy of Sciences, Theoretical Biology and Theoretical Ecology Researches Group

Specialization is a serious topic of evolutionary biology, with a lot of interesting problems related not only to the ecology of niche segregation or the "division of labour" in recent forms of life, but also to the field of prebiotic replicator evolution. The coexistence of catalytic replicators (information-carrying molecules with enzymatic activities) in the Hipercycle (Eigen and Schuster, 1971; Boerlijst and Hogeweg, 1991) or in the Metabolic replicator model (Czárán and Szathmáry, 2000; Könnyü et al.) is unthinkable without previous specialization processes leading to some kind of "enzyme specificity". The common assumption of these models is that every replicator type has a well-defined, specific function with which it contributes to the maintenance of the system. Thus, if any one of the cooperating replicator types is absent, the replicator community as a whole collapses due to the missing function. Both the Hypercycle and the Metabolic Replicator models are concerned with the problem of the coexistence of specialized replicators and their resistance to the attack of parasitic replicators which do not contribute to the common good at all, or even do explicit harm to the system. These models do not explain, however, why and how specialization comes about in a system of catalytic replicators. That is what we attempt in our present work.

This model is based on the Metabolic replicator system in which each replicator type is supposed to catalyze a specific reaction of a simple network of metabolism. Metabolism 
produces the monomers for the replication of all the replicators, thus it is necessary that the reactions of metabolism be catalyzed, otherwise the system dies out. To keep the system at its simplest form, we assume that the metabolic "network" is constituted by two chemical reactions (reaction A and B), and that the replicators can catalyze both these reactions at the beginning, $i$. e., the initial replicator population is that of "generalists". We also assume a trade-off relation between the two different enzymatic activities: a good catalyst of reaction A cannot be very good at catalyzing reaction $\mathrm{B}$, and vice versa. Another trade-off is assumed between enzymatic activity and replication rate: good enzymes cannot replicate very fast, and fast replicators cannot be good catalysts. Of course, fast and non-catalyzing replicators are the parasites of this system. We let the system of different generalists evolve on a two-dimensional cellular automaton, assuming that mutations (constrained by the unified trade-off function) can occur during replications. We search for parts of the parameter space of the model that allow for specialization (extreme evolutionary shift towards a mix of the two specialist types of replicators) and parasite resistance. We find that under certain conditions (i.e., at limited mobility of the replicators on the mineral surface, and for certain shapes and parameter regimes of the trade-off function) specialization and parasite resistance both occur in the metabolic system.

Boerlijst, M. C. and Hogeweg, P. (1991). Spiral wave structure in pre-biotic evolution: hypercycles stable against parasites. Physica D 48:17-28. Dieckmann, U., Law, R., and Metz, J. A. J. eitors (2000). The geometry of ecological interactions: simplifying spatial complexity. Czárán, T and Szathmáry, E. pp. 116-134. Eigen, M. and Schuster, P. (1979) The hypercycle. Springer-Verlag, Berlin.

Könnyü, B., Czárán, T. and Szathmáry, E. Prebiotic replicase evolution in a metabolic system. (submitted)

E-mail: konnyu@caesar.elte.hu

\section{Autopoietic Vesicles in Different Dynamic Regimes: Growth, Homeostasis and Decay}

Fabio Mavelli ${ }^{1}$, Pasquale Stano ${ }^{2,3}$

${ }^{1}$ Chemistry Department, University of Bari; ${ }^{2 "}$ Enrico Fermi" Study and Research Centre, Rome, Italy; ${ }^{3}$ Biology Department, University of RomaTre

Autopoiesis, as developed by Maturana and Varela in the seventies (Varela 1974, Maturana 1980, Fleischaker 1988, Luisi 2003), represents one of the most complete theories to represent the "blue print" of life. Originally developed as representation of cellular life, it poses as a main feature the self-maintenance of the cell, as due to a process of self-generation of the components from within the cellular boundary, a boundary which is itself a product from within. Thus, cellular life is seen as an organized network of processes, which has as a product its very organization. Different chemical implementations in the test tube has been presented during years all based on surfactant self-assembling structures, as micelles (Bachman 1992), reverse micelles (Bachman 1992) and vesicles (Walde 1994) which, as recently emphasized, can be defined as autopoietic but not as living, since autopoiesis being the necessary, but not the necessary and sufficient, condition for life (Bitbol 2004). More recently, Zepik et al. (Zepik 2001) successfully reported on the first experimental attempt to model chemical autopoietic structures in three different regimes: continuous growth, homeostasis and decay, by introducing a surfactant decay reaction in the well-known growth-division approach to vesicle selfreproduction.

In this contribution a simple mechanism that reproduce the behaviors modeled by Zepik et al. will presented and discuss. This mechanism will be studied both in a deterministic a 
stochastic approach using, for the latter one, a suitable Monte Carlo program recently developed by one of us (Mavelli 2006). The final aim is to show as very simple selfassembly supra-molecular structures can exhibit behaviors that mimic real cells and as they could play a key role in the emergence of life on Earth. in our simple model, A second but non minor goal is to elucidate the roles of random fluctuations in this pathway showing as they can act as a selection rule by selecting only the more robust organisms, that is in our simple model, allowing to survive only larger structures.

Bachmann PA, Luisi PL, Lang J (1992) Autocatalytic self-replicating micelles as models for prebiotic structures. Nature 357,57-59.

Bachmann PA, Walde P, Luisi PL, Lang J (1990) Self-replicating reverse micelles and chemical autopoiesis. J. Am. Chem. Soc. 112,8200-8201.

Bitbol M, Luisi PL (2004) Autopoiesis with or without cognition: defining life at its edge. J. R. Soc. Interface 1,99-107.

Fleischaker GR (1988) Autopoiesis: The status of its system logic. Biosystems 22,37-49.

Luisi PL (2003) Autopoiesis: A review and a reappraisal. Naturwissenschaften 90, 49-59. Maturana HR, Varela FJ (1980) Autopoiesis and cognition-The realization of the living. Reidel, Dordrecht (Holland).

Mavelli F., Piotto S. (2006) Stochastic Simulations of Homogeneous Chemically Reacting Systems. Journal Of Molecular Structure-THEOCHEM. 771, 55-64.

Varela FJ, Maturana HR, Uribe R (1974) Autopoiesis: The organization of living systems, its characterization and a model. Biosystems, 5,187-196.

Walde P, Wick R, Fresta M, Mangone A, Luisi PL (1994) Autopoietic self-reproduction of fatty acid vesicles. J. Am. Chem. Soc. 116,11649-11654.

Zepik H.H., Blöchliger E., Luisi P.L. A (2001) Chemical Model of Homeostasis Angew. Chemie 1, 199-202.

E-mail: mavelli@chimica.uniba.it

\section{Selective Interactions Between RNA and Lipid Vesicles}

\section{Erica D’Aguanno, Chris Thomas, Pasquale Stano, Pier Luigi Luisi}

Biology Department -University of RomaTre, Rome, Italy

RNA and vesicles are two important molecular classes in the origin of life and early evolution, but they are not generally considered as interacting partners. Very recently, three reports (cited below) have make clear that the interaction between these two molecular systems may lead to behaviour (selection, competition) which are typical of protocell populations. In the most important case (Thomas and Luisi, 2005) it was shown by us that t-RNA may select cationic vesicles according to their size. In particular, small vesicles did not precipitate in the presence of negatively charged RNA, whereas large vesicles did. This process has been indicated as an example of primitive protocell selection. We show, together with a brief comment on our initial report, some new aspects of interactions between nucleic acids and lipid vesicles.

Chen IA, Roberts RW, Szostak JW (2004) The emergence of competition between model protocells. Science 305, 1474.

Cheng, Z.; Luisi, P. L. (2003) Coexistence and Mutual Competition of Vesicles with Different Size Distributions. J. Phys. Chem. B 107, 10940. 
Thomas CF, Luisi PL (2005) RNA selectively interacts with vesicles depending on their size. J Phys Chem B.109, 14544.

E-mail: luisi@mat.ethz.ch

\section{Theoretical Approaches to the Ribocell}

Fabio Mavelli ${ }^{1}$, Pier Luigi Luisi ${ }^{2}$

${ }^{1}$ Chemistry Department, University of Bari; ${ }^{2}$ Biology Department, University of RomaTre

The Ribocell is an hypothetical cellular model that has been proposed some years ago as a minimal cell model (Szostak et al 2001). It consists in a self-replicating minimum genoma coupled with the self-reproduction of the lipid vesicle where it is contained. This model assumes the existence of two hypothetical ribozymes one able to catalyze the conversion of molecular precursors into lipids and the second one able to translate and duplicate RNA strands. Therefore, in an environment rich of lipid and activated nucleotide precursors the ribocell can self-reproduce if the two mechanism: the genoma self-replication and the membrane reproduction (growth and splitting) are somehow synchronized. The aim of this contribution is to explore the feasibility of this models starting from the assumption that all the involved processes can be efficient as needed. In particular, the questions we asked are: under the best experimental conditions, can the ribocell reaches a stationary condition where it oscillates continuously between two states after an before the splitting? Is there a concentration threshold for the genetic material to avoid that the daughters cell remain without the minimal genetic kit to be alive? Or, in other worlds, how much is this model robust to random fluctuations? We try to answer to these questions in the perspective of the more general problem of building up a minimal cell (Luisi et al. 2006a, b) coupling an internal metabolic network that produce lipids (Mavelli \& Ruiz-Mirazo 2006) with the dynamics of the vesicle membrane (Mavelli \& Ruiz-Mirazo 2007a, b).

Luisi, P.L., Chiarbelli, C, Stano, P. (2006b). From Never Born Proteins to Minimal Living Cells: Two Projects in Synthetic Biology. Orig.Life Evol. Biosphere 36, 605-616.

Luisi, P.L., Ferri, F, Stano, P. (2006a). Approaches to semi-synthetic minimal cells: a review. Naturwissenschaften 93, 1-13.

Mavelli F., Ruiz-Mirazo, K. (2006) Stochastic simulations of minimal self-reproducing cellular systems. Phil. Trans. R. Soc. B, 362, 1789-1802.

Mavelli, F., Ruiz-Mirazo, K. (2007a). Bridging the gap between in vitro and in silico approaches to minimal cells. Orig.Life Evol. Biosphere 37, 455-458.

Mavelli, F., Ruiz-Mirazo, K. (2007b). Stochastic Simulation of fatty-acid proto cell models. In: Sergey M. Bezrukov, editor, Noise and Fluctuations in Biological, Biophysical, and Biomedical Systems. vol. 6602, pages: 1B1-1B10. SPIE Bellingham, Washington.

Szostak, J.W., Bartel, D.P., Luisi, P.L. (2001). Synthesizing life. Nature, 409, 387-390.

E-mail: mavelli@chimica.uniba.it

\section{The Origin of nTP: GTP for Information and ATP for Energy}

\section{Ken Naitoh}

Waseda University, Faculty of Science and Engineering, Tokyo, Japan

The reason why adenosine triphosphate (ATP) is naturally selected as the main energycarrier is not clarified. (Duve 2005) We examined the databases (Benson 2003, Lowe 1997, 
Nakamura 2000, DNA databank of Japan, JCM On-line catalogue) in order to clarify whether guanosine triphosphate (GTP) is mainly used as information storage in ribonucleic acids (RNAs), because adenine-uracil (A-U) pair in weaker connections would be dropped out relatively among candidates of information carriers. Actual frequencies of G-C pairs in the RNAs of hyper-thermophiles are much more than those of A-U pairs. (Naitoh 2005) The A-U pairs are less than G-C pairs also in RNAs of microorganisms such as Yeast preferring lower temperatures. Thus, we propose the simple hypothesis that the rates of adenine $(A)$ and uracil $(\mathrm{U})$ relatively lower than the rate of guanine $(\mathrm{G})$ and cytosine $(\mathrm{C})$ in RNAs bring redundant monomers of ATP and UTP outside nucleic acids, which become energy-carriers under unavoidable circumstances. (Naitoh, 2008) ATP run off from RNA is something like the joker in the card-game of old maid. The other redundant uridine triphosphate (UTP) became polysaccharide-generator. Possible answers were given also for the questions why two types of nitrogenous bases, large purine and small pyrimidine, are used for nucleic acids and also why only twenty types of amino-acids are employed for proteins. (Naitoh 2001, 2006) Physical thought experiment may bring us the possible overall scenario explaining the origin of nitrogenous bases and nucleic acids.

Benson, D.A., et al (2003) GenBank. Nucleic Acids Res. 31: 23-7.

de Duve, C. (2005) Singularity: Landmarks on the pathways of life, Cambridge University Press.

DNA Data Bank of Japan, (“http://www.ddbj.nig.ac.jp/”)

JCM On-line catalogue, Japan Collection of Microorganisms, RIKEN, "http://www.jcm. riken.go.jp/".

Lowe, T.M., \& Eddy, S.R. (1997) tRNAscan-SE: a program for improved detection of transfer RNA genes in genomic sequence. Nucleic Acids Res., 25: 955-64. (available at "http://rna.wustl.edu/tRNAdb/")

Nakamura, Y., Gojobori, T., \& Ikemura, T. (2000) Codon usage tabulated from the international DNA sequence databases. Nucl. Acids Res., 28: 292. (available at "http:// www.kazusa.or.jp/codon/")

Naitoh, K.(2001) Cyto-fluid Dynamic Theory, Japan Journal of Industrial and Applied Mathematics, 18-1: 75-105.

Naitoh, K.(2005) Self-organising mechanism of biosystems, Journal of Artificial Life and Robotics, 9: 96-98.

Naitoh, K. (2006) Gene engine and machine engine, Springer-Japan.

Naitoh, K. (2008) Inevitability of nTP, Information-energy carrier, Proceedings of 13th International Symposium on Artificial Life and Robotics.

E-mail: k-naito@waseda.jp

\section{FeS Surface Dynamics \& Molecular Evolution}

\section{Andrew J, Pratt*, Vladimir Golovko, Henry Toombs-Ruane}

\section{Department of Chemistry, University of Canterbury, New Zealand}

In accordance with Mike Russell's model for the origin of life at alkaline hydrothermal vent systems (Martin and Russell, 2003) iron-sulfur mineral systems mediate a wide variety of processes that are required for the origin of metabolism and hence life on earth: they provide a continuous input of redox energy; and catalyse a range of transformations that mimic extant FeS-dependent processes of anaerobic metabolism including carbon (Huber 
and Wächtershäuser, 1997) and nitrogen (Dörr et al., 2003) fixation reactions. Furthermore, iron mineral precipitates catalyse biomimetic phosphoryl-transfer processes, including the generation and accumulation of polyphosphates (de Zwart et al., 2004).

If mineral precipitates were important in the early establishment of biochemistry, as suggested by these results, how might fully soluble biochemical metabolism emerge? Experimental results on the dynamics of surface binding of metabolites on iron sulfide minerals will be presented. The binding of a range of ligands, including phosphates and thiols, to iron sulfide minerals have been evaluated. The binding is competitive and organic derivatives are selectively displaced from the bulk surface. The dynamic solvation processes are compatible with selective accumulation of biochemically significant species in the supernatant (Baaske et al., 2007). These processes in a microporous hydrothermal mineral environment can provide both solution autocatalytic chemistry and a backdrop of homeostasis. These results are incorporated into a model for the emergence of metabolism as a property of autocatalytic processes that dissipate a thermochemical gradient and which are localized within microporous compartments. Inheritable reproduction and variation of such discrete autocatalytic processes, with selection for more efficient catalysis and enhanced reaction dynamics, provides the basis for Darwinian selection to arise at a molecular level thus seeding the emergence of a protometabolic foundation for life.

Baaske P., Weinert F. M., Duhr S., Lemke K. H., Russell M. J., and Braunde D. (2007) Extreme accumulation of nucleotides in simulated hydrothermal pore systems. Proc. Natl. Acad. Sci USA, 104: 9346-9351.

Dörr M. KäëŸbohrer J., Grunert R., Kreisel G., Brand W. A., Werner R. A., Geilmann H., Apfel C., Christian Robl C. and Weigand W. (2003). A possible prebiotic formation of ammonia from dinitrogen on iron sulfide surfaces. Angew.Chem. Int. Edn. Engl. 42: 1540-1543.

Huber C. and Wächtershäuser G. (1997). Activated Acetic Acid by Carbon Fixation on (Fe, Ni)S Under Primordial Conditions. Science 276: 245-247.

Martin W. and Russell M. J. (2003). On the origins of cells: a hypothesis for the evolutionary transitions from abiotic geochemistry to chemoautotrophic prokaryotes, and from prokaryotes to nucleated cells. Phil. Trans. R. Soc. B 358: 59-83.

Zwart I. I., Meade S. J. and Pratt A. J. (2004). Biomimetic phosphoryl transfer catalysed by iron(II)-mineral precipitates. Geochim. Cosmochim. Acta 68: 4093-4098.

E-mail: andy.pratt@canterbury.ac.nz

\section{Molecular Evolution of the Interaction Between Prophage Genes and Their Prokaryotic Hosts: The Case of Sulfolobus spp}

Yetzi Robles, Arturo Becerra, Antonio Lazcano

Facultad de Ciencias, UNAM Apto. Postal 70-407, Ciudad Universitaria, México, D. F. 04510, México

In order to understand the evolutionary dynamics between bacteriophages and their prokaryotic hosts in terms of gene transfer and their maintenance in viral and hosts genomes, a comparative study was carried out. Two data bases were created with viral and celular genomes available in public data bases. Sequence comparisons were performed using BLAST between both data bases to identify homologs between viral and hosts proteins. Two distinctive groups of homologous proteins were thus identified: (a) a group of hypothetical proteins that are present in viral genomes that infect archaea 
and their homologs found in archaeal genomesonly; and (b) a second group of hypothetical proteins that are present in several bacteriophage genomes and viruses that infect archaea, whose homologs were found in genomes of all three domains of life. In cases the proteins functions were predicted, most of which included functions related to nucleotide synthesis and amino acid metabolism, although interesting cases were found like that of a probable protein involved in polysaccharide biosynthesis and a colagenase. It is concluded that the identified sequences may lay a role favouring the production of viral particles infecting archaea.

E-mail: yetzi1980@hotmail.com

\section{Dynamics of Pattern Formation in Biomimetic Systems}

F. Rossi ${ }^{1 *}$, S. Ristori ${ }^{2}$ M. Rustici ${ }^{3}$, N. Marchettini ${ }^{4}$, E. Tiezzi ${ }^{4}$

${ }^{1}$ Dipartimento di Chimica Fisica, Universit di Palermo, Italy; ${ }^{2}$ Dipartimento di Chimica, Universit di Firenze, Italy; ${ }^{3}$ Dipartimento di Chimica, Universit di Sassari, Italy; ${ }^{4}$ Dipartimento di Scienze e Tecnologie Chimiche e dei Biosistemi, Universit di Siena, Italy

Cellular organization involves a complex interaction among structure, chemical kinetics, and transport processes. By using model systems where these features can be controlled to a large extent independently of the others, the relative contribution of each aspect to cellular attributes can be inferred.

The Belousov-Zhabotinsky (BZ) (Belousov 1958; Zhabotinsky 1964) reaction spontaneously produces complex spatial patterns (spirals, spots,...) that may oscillate in time or remain stationary and for this property it can be considered a valid model for self structuring and self patterning phenomena. Insights gained from the study of the BZ reaction carried out in biomietic matrices may shed light on the emergence of shape in living systems. For example these systems can be used to investigate the occurrence of selforganized patterns in media confined at the nano- to micromicrometer scale, and/or to design a chemical oscillator composed of biological molecules.

The route followed to develop these ideas was to couple chemical oscillations produced by $\mathrm{BZ}$ reaction with confined reaction environments such as direct and reverse micelles (Federico Rossi et al. 2008; Vanag \& Epstein 2008) and phospholipids bilayers (Magnani et al. 2004; Ristori et al. 2007); confinement being an essential requirement for any process of Life. Special focus was placed on systems which also present organic or lipidic compartments, as more reliable biomimetic matrices.

Belousov, B.P., 1958. A periodic reaction and its mechanism. In A Periodic Reaction and its mechanism. Moscow: Medgiz, pagg. 145-147.

Magnani, A. et al., 2004. Chemical waves and pattern formation in the 1,2-dipalmitoyl-snglycero-3-phosphocholine/water lamellar system. Journal of the American Chemical Society, 126(37), 11406-11407.

Ristori, S. et al., 2007. Interplay between the Belousov-Zhabotinsky reaction-diffusion system and biomimetic matrices. Chemical Physics Letters, 436, 175-178.

Rossi, F. et al., 2008. Spatio-Temporal Perturbation of the Dynamics of the Ferroin Catalyzed Belousov-Zhabotinsky Reaction in a Batch Reactor Caused by Sodium Dodecyl Sulfate Micelles. Journal of Physical Chemistry B, 112, 7244-7250.

Vanag, V.K. \& Epstein, I.R., 2008. Patterns of Nanodroplets: The Belousov-ZhabotinskyAerosol OT-Microemulsion System. In Self-Organized Morphology in Nanostructured 
Materials. Springer Series in Materials Science. Berlin: K. Al-Shamery and J. Parisi, eds., pagg. 89-113.

E-mail: f.rossi@unipa.it

\title{
Metabolism First Theories: An Evaluation
}

\author{
Robert Shapiro
}

Department of Chemistry, New York University, New York, N.Y., USA

The most significant division between theories suggesting a mechanism for the origin of life may be the one between the "metabolism-first" and "replicator first" points of view. The latter proposal has been favored among the majority of scientists in the field for several decades. It requires, however, the spontaneous assembly by abiotic chemical processes of a macromolecule that can catalyze its own self-replication. Such an event would be extremely improbable, and the theory implies that life may be exceedingly rare in this universe (Shapiro, 2000).

The competing position, metabolism first, has lesser requirements: a mixture of smaller organic molecules such as those found in carbonaceous meteorites, a solvent suitable for the support of chemical reactions of these molecules, and an interactive energy source to drive the process of self-organization (Morowitz, 1968; Feinberg and Shapiro, 1980). This concept has often been described in terms of an autocatalytic reaction cycle, in which sufficient quantities of carbon dioxide or simple organic molecules are absorbed in each turn of the cycle to double the amount of material within it. The participating members of the cycle also serve as catalysts for the reactions of the cycle (Kauffman, 1994). Variants of the reductive citric acid cycle have often been cited as possible examples of such a cycle (Wchtershuser, 1990; Morowitz, 1999).

Several recent papers have challenged the plausibility of such schemes on a number of grounds (Pross, 2004; Orgel, 2008). They have argued that specific catalysis of cycle reactions by its members is implausible; that many competing reactions would draw off material and disrupt the cycle and that no driving force had been specified that would favor the spontaneous self-organization of a disordered system. No experimental demonstration of the operation of such a system has been made. I will argue that the first three objections can be remedied if an external energy source can be coupled specifically to a reaction of the central cycle. Thermodynamic factors would then favor the central cycle and draw organic material from competing reactions into it; no specific catalysis would be required. Environmental changes could lead to the evolution of the central cycle into a more complex self-sustaining reaction network (Shapiro, 2006).

An experimental "proof of principle" reaction will be needed, however, to validate this concept. Suggestions will be made about about the design of such a demonstration and of plausible components for the initiation of such a cycle.

Feinberg, G. and Shapiro, R. (1980). Life Beyond Earth. Morrow, New York.

Kauffman, S. (1994) At Home in the Universe. Oxford Univ. Press, New York

Morowitz, H J. (1968).. Energy Flow in Biology. Academic Press, New York.

Morowitz, H J. (1999). A theory of biochemical organization, metabolic pathways, and evolution. Complexity, 4: 39-53.

Orgel, L.E. (2008). The Implausibility of Metabolic Cycles on the Prebiotic Earth. PloS Biology, 6: 5-13. 
Pross A. (2004). Causation and the origin of life: metabolism or replication first? Origins Life Evol. Biosphere, 34: 307-321.

Shapiro, R. (2000). A replicator was not involved in the origin of life. IUBMB Life, 49: 173-176.

Shapiro, R. (2006). Small molecule interactions were central to the origin of life. Quarterly Review of Biology, 81: 105-125.

Wchtershuser, G. (1990). Evolution of the first metabolic cycles. Proc. Natl. Acad. Sci. USA, 87: 200-204.

E-mail: rs2@nyu.edu

\title{
The Role of Interpretation in the Emergence of Life
}

\author{
Christopher Southgate, Andrew Robinson
}

University of Exeter, UK

One of the most fundamental properties of living organisms is what might most generally be called 'interpretation' - organisms process their environment, make (fallible) interpretations of it in such a way as to improve their chance of flourishing and reproducing. A classic example often cited is that of the hungry bacterium that detects a glucose molecule and swims in the direction from which it came (Kauffman 2000). In other work we have sought to provide a precise definition of this property that would apply to every type of interpretation from the most primitive to that of a conscious agent (Robinson and Southgate 2008). Essential to this definition is that the property of interpretation, though fully explicable in naturalistic terms, be non-reducible to a sequence or complex of merely mechanical effects.

What we propose is that interpretation may occur in proto-biotic systems, and that detection of such a property in model systems would provide a positive indication of the plausibility of such systems as candidates for precursors of life. The problems with such systems will be well known to conference participants, and include how reagents can remain sufficiently localised to interact, and how systems acquire a replicable identity that can be subject to natural selection. Although we are well aware of the problems of RNAbased model systems (Orgel 2002), we also recognise the promising work that has been done in such systems (Ferris 2005; Johnston 2001). Our first model system for testing will therefore be a population of RNA hairpin loops, localised by adsorption on a surface, and exposed to pulses of activated nucleotides. It is intuitively obvious that a hairpin loop that opened in response to first contact with, say, a single activated nucleotide (as opposed to opening only by chance, or in response to a large number of nucleotides) would have the maximum chance of taking advantage of the pulse and being replicated. We are developing computer-modelling procedures to substantiate this intuition. Such behaviour would constitute in our terms an interpretation of the environment. Successful interpretations will lead to particular sequences tending to dominate in the population. Although the simulation of such a pulsed system contains arbitrary assumptions about pulse-length and substrate concentration, all other parameters could be set with reference to known physicochemical data (e.g. Xia et al 1999). The 'melting phase' of such abiotic replication presents problems which have not yet yielded to experimental modelling. However from the point of view of our computer modelling the melting phase may be taken to be a constant across interpreting and non-interpreting systems

We also consider in our paper how different models of the origin of life might relate to one another, by considering the 'probable next evolutionary step' by which different types of 
model systems might be expected to progress towards the complete set of properties possessed by living organisms. For example, our own 'minimal interpreting entity' would acquire substantially increased selective advantage by evolving the properties of autocatalysis and the capacity to perform a thermodynamic work-cycle. We repeat this analysis with the autocell proposal (Deacon 2006), vesicle models (Deamer 1997) and the Kauffman hexamertrimer system (Kauffman 2000; Kauffman and Clayton 2006), showing in each case how the acquisition of the property of interpretation would confer a selective advantage.

Extension of our focus on interpretation will include consideration of how vesicles might develop interpretation via differential pore formation, and further exploration of RNA hairpin loops. We are particularly interested in the possibility that amino-acyl nucleoside monophosphates could have functioned as prebiotic activated nucleotides, and that this might account for the first coupling of RNAs with peptide formation, and for the persistence of aminoacyl-AMPs as biological intermediates.

DEACON, T. W. (2006) Reciprocal Linkage between Self-organizing Processes is Sufficient for Self-reproduction and Evolvability. Biological Theory, 1, 136-149.

DEAMER, D. W. (1997) The First Living Systems: a Bioenergetic Perspective. Microbiology and Molecular Biology Reviews, June, 237-61.

FERRIS, J. P. (2005) Catalysis and Prebiotic Synthesis. Reviews in Mineralogy and Geochemistry, 59, 187-210.

JOHNSTON, W. K., UNRAU, P. J., LAWRENCE, M. S., GLASNER, M. E. \& BARTEL, D. P. (2001) RNA-Catalysed RNA Polymerization: Accurate and General RNA-Templated Primer Extension. Science, 292, 1319-25.

KAUFFMAN, S. A. (2000) Investigations, Oxford, Oxford University Press.

KAUFFMAN, S. \& CLAYTON, P. (2006) On emergence, agency, and organization. Biology and Philosophy, 21, 500-520.

ORGEL, L. E. (2002) The Origin of Biological Information. IN SCHOPF, J. W. (Ed.) Life's Origin: The Beginnings of Biological Information. Berkeley, University of California Press. ROBINSON, A.J. and SOUTHGATE, C. (2008) 'Interpretation and the Emergence of Life', submitted to Biology and Philosophy.

XIA, T., MATHEWS, D. H. \& TURNER, D. H. (1999) Thermodynamics of RNA Secondary Structure Formation. IN SÖLL, D., NISHIMURA, S. \& MOORE, P. B. (Eds.) Comprehensive Natural Products Chemistry: Vol. 6-Prebiotic Chemistry, Molecular Fossils, Nucleosides and RNA. Amsterdam, Elsevier.

E-mail: c.c.b.southgate@ex.ac.uk

\section{Interaction of Amino Acids with Clay Minerals and Their Relevance to Chemical Evolution and the Origins of Life}

Frankie Sami, Brij Bhushan Tewari

Department of Chemistry, Faculty of Natural Sciences, University of Guyana, P.O. Box: 101110, Georgetown, Guyana

A model is proposed for a prebiotic environment in which concentration, condensation and chemical evolution of biomolecules could have taken place. Clays are likely to have been among the most important minerals because of their relatively large surface-area-to-volume ratio, catalytic properties and wide spread geological occurrence. Chemical reactions on mineral surfaces (Bernal, 1949) may have provided a prebiotic route to the biopolymers 
required for the first life on the primitive earth since the larger polymers bind more strongly on the mineral surface.

Adsorption of dl-aspartic acid, dl-leucine, dl-lysine and dl-serine in aqueous solution on halosite, hectorite, illite, kaolinite, nantronite and montmorillonite is described. Interaction was studied at neutral $\mathrm{pH}(7.1+0.01)$ and room temperature $\left(30+1^{\circ} \mathrm{C}\right)$. The progress of adsorption was followed spectrophotometrically by measuring the absorbance of amino acids solution at their corresponding $\lambda_{\max }$. Leucine and aspartic acid are found to have maximum and minimum adsorption respectively on all clay minerals studied. The Laugmuir type of adsorption is followed in the concentration range $10^{-3}-10^{-4} \mathrm{M}$ of amino acids solution. Amino acids and mineral surfaces are considered to have played important role in peptide formation during the course of chemical evolution in the primeval sea.

Bernal, J. D. (1949) Proc.Roy.Soc.London, 357A: 537-558.

XIA, T., MATHEWS, D. H. \& TURNER, D. H. (1999) Thermodynamics of RNA Secondary Structure Formation. IN SÖLL, D., NISHIMURA, S. \& MOORE, P. B. (Eds.) Comprehensive Natural Products Chemistry: Vol. 6-Prebiotic Chemistry, Molecular Fossils, Nucleosides and RNA. Amsterdam, Elsevier.

E-mail: brijtew@yahoo.com

\title{
Molecular Dynamics in Nanopores and the Origin of Life
}

\author{
Richard E. Wilde
}

\section{Department of Chemistry and Biochemistry, Texas Tech University}

The vibrational dephasing dynamics of methyl iodide in silica sol gel nanopores have given an insight into the behavior of molecules trapped in mesopores. These studies have utilized the Fourier transform of Raman band shapes to produce vibrational correlation and memory functions. These functions have been analyzed by time series analysis using Zwanzig-Mori formalism to establish homogeneous and inhomogeneous contributions to the spectral second moments. The conclusions of this research will be described, and the significance of these contributions to molecular dynamics and chemical reactions in nanopores will be discussed. The connection will then be made to the influence of nanopores in the chemistry at ancient hydrothermal vents and how this chemistry can account for the appearance of the first life-forming chemicals. Experiments that have been designed to discover this early chemistry will be discussed.

E-mail: richard.wilde@ttu.edu

\section{A Robust Pathway for Protocell Growth and Division Under Plausible Prebiotic Conditions}

Ting F. Zhu ${ }^{1,2}$, Jack W. Szostak ${ }^{1}$

${ }^{1}$ Howard Hughes Medical Institute, and Department of Molecular Biology, Massachusetts General Hospital, Boston, Massachusetts 02114, USA; ${ }^{2}$ Harvard-MIT Division of Health Sciences and Technology, Massachusetts Institute of Technology

A primitive cell must comprise two fundamental components: a self-replicating genome, and a membrane compartment (vesicle) that can grow and divide. In this study, we show 
that one of these two fundamental components, a membrane compartment that can grow and divide, may emerge under model prebiotic conditions. We show that fatty acid vesicles, by simple feeding with fatty acid micelles, can grow into thread-like shapes through a series of dramatic shape transformations. These thread-like vesicles, under the influence of mild fluid perturbations, can divide into multiple daughter vesicles, each inheriting the encapsulated genetic molecules of their parent vesicle. In modern life, cell division is a process which requires highly sophisticated protein machinery to accomplish. Our results demonstrate how, without complex proteins, an artificial membrane compartment can grow and divide under simple prebiotic conditions.

Chen, I. A., Roberts, R. W. \& Szostak, J. W. The emergence of competition between model protocells. Science 305, 1474-6 (2004).

Hanczyc, M. M., Fujikawa, S. M. \& Szostak, J. W. Experimental models of primitive cellular compartments: encapsulation, growth, and division. Science 302, 618-22 (2003). Hanczyc, M. M. \& Szostak, J. W. Replicating vesicles as models of primitive cell growth and division. Curr Opin Chem Biol 8, 660-4 (2004).

Szostak, J. W., Bartel, D. P. \& Luisi, P. L. Synthesizing life. Nature 409, 387-90 (2001).

E-mail: tzhu@mit.edu 


\section{Astrobiology and Search for Life}

\section{Geochemical Testbed Research for Life Detection on Mars}

Andrew D. Aubrey ${ }^{1}$, Frank J. Grunthaner ${ }^{1}$, Max L. Coleman ${ }^{1}$, Mark A. Sephton ${ }^{2}$, John H. Chalmers $^{3}$, Jeffrey L. Bada ${ }^{3}$

${ }^{1}$ Jet Propulsion Laboratory and California Institute of Technology, Pasadena, CA; ${ }^{2}$ Imperial College London, UK; ${ }^{3}$ Scripps Institution of Oceanography, La Jolla, CA

The Urey Instrument is an advanced in situ instrument suite designed to assess the presence of organics and oxidants on the Martian near surface (Aubrey et al., 2008). Ultra-high sensitivity to primary amines is achieved after a two-stage extraction using sub-critical water (SCWE) and sublimation (MOD) followed by quantification of fluorescent derivatives after separation of target compounds via $\mu$-capillary electrophoresis. Using these methods, parts-per-trillion (pptr) sensitivity is achieved $\left(10^{3}\right.$ cells/g) and can be correlated to the presence of oxidants within the Martian regolith using the Mars Oxidant Instrument (MOI). The biomolecules targeted by Urey include amino acids, nucleobases, and amine degradation products that may be present due to extinct or extant biological activity. Measurements of amino acid chirality provide a method to discriminate between abiotic and biological molecules, as L-enantiomer dominated amino acid compositions are recognized as definitive biosignatures (Kvenvolden, 1973).

Instruments such as $\boldsymbol{U} \boldsymbol{r e y}$ for in situ Mars exploration must be thoroughly tested using relevant terrestrial samples representative of Mars environments with respect to geochemistry, mineralogy, and concentrations of target bioorganic compounds. The Astrobiology Sample Analysis Program (ASAP) showed the scientific ramifications of instruments working in parallel to well characterize a subset of Mars analog samples by various flight instruments (Glavin et al., 2008). ASAP represents the conception of an inclusive sample library that can be used as a testbed for in situ instrumentation for future Mars exploration. Martian analog samples can be selected based on a wide range of physical and chemical criteria (Marlow et al., 2008), so it is important that a set of analog samples be designated specifically for life detection missions. This library must contain terrestrial environmental samples analogous not only to the soil and rock chemistries detected in situ by the Mars Exploration Rovers (MER), but also to the mineralogical classes remotely sensed by orbiting spacecraft instrumentation (OMEGA, CRISM), such as sulfates and phyllosilicates. Most importantly, this group of samples must include organic matter representing various diagenetic states that range from extant microbial communities to heavily degraded organic compounds. The viability of Mars life detection instrumentation must be evaluated based on the ability to characterize biomarkers that provide unequivocal evidence of life within these Mars analog samples with respect to sensitivity, mineralogy, and diagenetic states of organic compounds.

As mission landing sites are often selected only months before launch, it is important that flight instruments demonstrate their function on a wide range of Mars analog geological samples for the purposes of instrument development, calibration, data acquisition, and interpretation. This study will share the strategy implemented for ongoing Urey instrument testbed research with respect to justification of an environmental sample library and developmental testing phases. This thorough testbed research will allow for the determination of matrix-specific optimization for analytical extraction conditions 
and the best chance at detecting remnants of an extinct or extant Martian biota during ExoMars 2013 as part of the Pasteur payload.

Aubrey, A. D., et al. (2008). The Urey Instrument: An Advanced in situ Organic and Oxidant Detector for Mars Exploration. Astrobiology, in press.

Glavin, D. P., et al. (2008). Astrobiology Sample Analysis Program (ASAP) for Advanced Life Detection Instrumentation Development and Calibration. Abscicon Abstract \#2-05-O. Astrobiology 8(2): 297.

Kvenvolden, K. A. (1973). Criteria for distinguishing biogenic and abiogenic amino acids - preliminary considerations. Space Life Sci., 4:60-68.

Marlow, J. J., Martins, Z., and Sephton, M. A. (2008). Mars on Earth: soil analogues for future Mars missions. Astron. Geophys., 49:2.2-2.5.

E-mail: Andrew.D.Aubrey@jpl.nasa.gov

\title{
Exposure of Amino Acids on the International Space Station: EXPOSE-Eutef and EXPOSE-R
}

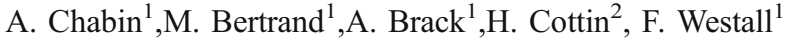 \\ ${ }^{1}$ Centre de Biophysique Moléculaire, CNRS, rue Charles Sadron 45072 Orléans Cedex 2, \\ France; ${ }^{2}$ LISA, Université Paris 7 \& Paris 12, UMR 7583 CNRS, Avenue du Général de \\ Gaulle, 94010 Créteil cedex, France
}

Space technology in Earth orbit offers a unique opportunity to study the behavior of amino acids required for the emergence of primitive life. We are therefore interested in the behaviour of amino acids in space conditions and their safe delivery to the primitive Earth. For more than a decade, our team has been carrying out experiments in space, testing the stability of amino acids, their derivatives, and small peptides that are exposed to solar UV either in the free state or mixed with finely ground meteorite material using. Two experiments were performed on board on Soyouz: Biopan I (Barbier, et al. 1998) and Biopan II (Barbier, et al. 2002), and on the Mir Station Perseus mission (Boillot, et al. 2002).

We presently have two experiments on the International Space Station: EXPOSE-Eutef and EXPOSE R. Proteic and non-proteic amino acids, as well as a dipeptide, were deposited either free or mixed with ground meteorite, as dry films behind $\mathrm{MgF}_{2}$ windows which are transparent to solar UV.

The space experiments are supported by experimental ground studies that are necessary in preparation and in support of these experiments. Although it is clear that we cannot accurately reproduce the space environment in the laboratory, we have used two irradiation chambers to partially simulate the effects of solar radiation on the same materials exposed to space (Cottin, et al. in press). The simulation chamber at the CBM-Orléans and at the DLR-Cologne use different wavelengths. We irradiated the samples for 15-30 days. After irradiation, the molecules were extracted, derivatised and analysed by GC-MS following chiral and non-chiral procedures. (Bertrand et al. 2008).

We present the different preparation steps of samples for the EXPOSE missions and the first analytical results of the ground experiments.

Barbier. B., Chabin, A., Chaput, D., and Brack, A. (1998). Photochemical processing of amino acids in Earth orbit. Planet. Space Sci., 46: 391-398. 
Barbier, B., Henin, O., Boillot, F., Chabin, A., Chaput, D., and Brack, A. (2002) Exposure of amino acids and derivatives in the Earth orbit. Planet. Space Sci., 50:353359.

Bertrand, M., Chabin, A., Brack and Westall, F. (2008) Separation of amino acid enantiomers VIA chiral derivatization and non-chiral gas chromatography. Journal of Chromatography, A 1180: 131-137.

Boillot, F., Chabin, A., Buré, C., Venet, M., Belsky, A., Bertrand-Urbaniak, M., Delmas, A., Brack, A., and Barbier, B. (2002) The Perseus Exobiology Mission on MIR: Behaviour of amino acids and peptides in Earth orbit. Origins of Life and Evolution of the Biosphere, 32: 359-385.

Cottin, H., Coll, P., Coscia, D., Fray,; N., Guan, Y.Y., Macari, F., Raulin, F., Rivron, C., Stalport, F., Szopa, C., Chaput, D., Viso, M., Bertrand, M., Chabin, A., Thirkell, L., Westall, F., and Brack A, (in press) Heterogenous solid/gas chemistry of organic compounds related to comets, meteorites, Titan, and Mars: Laboratory and in lower Earth orbit experiments. To appear in the Adv. Space Res.

E-mail: annie.chabin@cnrs-orleans.fr

\section{Experimental Fossilization Induced in Modern Microbial Mats}

Elizabeth Chacón $\mathrm{B}^{1}$, Mariajose Peña ${ }^{1}$, Felipe Torres de la Cruz ${ }^{1}$, A. Negrón-Mendoza ${ }^{2}$

${ }^{1}$ Facultad de Ciencias de la Tierra, UANL; ${ }^{2}$ Instituto de Ciencias Nucleares, UNAM

Microbial fossilization is a key geobiological process to understand the sedimentary record and to design new strategies in the extraterrestrial life search. Although several analysis have been proposed to identify and describe in situ fossilization of different types of microorganisms (Jones et al 1999; Westfall et al 2001), the many factors involved in this complex process still wait for elucidation. By far, the most common microbial fossil preservation process is by silicification, as the numerous ancient cyanobacterial microfossils from Precambrian strata testify. Other less common fossilization processes include phosphate and carbonate replacement. Among the main factors inducing fossilization are a rapid lithification, a rapid burial after cell death, cooling and evaporation of supersaturated mineral waters (mainly in the case of silicification) as well as the biological mediation on the nucleation of specific minerals input from the environment (Konhauser et al 2001). Previous works have suggested that biological organic matter mediates biomineralization; in contrast, other recent observations indicate that mineralization of cyanobacteria is an inorganically controlled process, induced by rapid cooling and evaporation of the spring waters, occurring independent of microorganisms. The aim of this work is to show some induction-experiments of microbial mats fossilization to investigate basic textural changes at the sedimentological level. The microbial mats under study come from the mesothermal sulfurous springs of the protected area of Baño San Ignacio in Linares, Nuevo Leon, Mexico. These microbial mats show a well-developed stratification and contain a high and complex diversity of microbial life. Microbial fossilization is induced on the surface of these mats by mineralization, irradiation, and sequential dehydratation steps. Some preliminary results after these fossilization experiments are changes in microfabric, texture, color, porosity and changes in the precipitates/biofilm ratio. These results are relevant not only in the context of the Earth's geobiological evolution but also in the search of potential biosignatures in astrobiology.

E-mail: liz@nucleares.unam.mx 


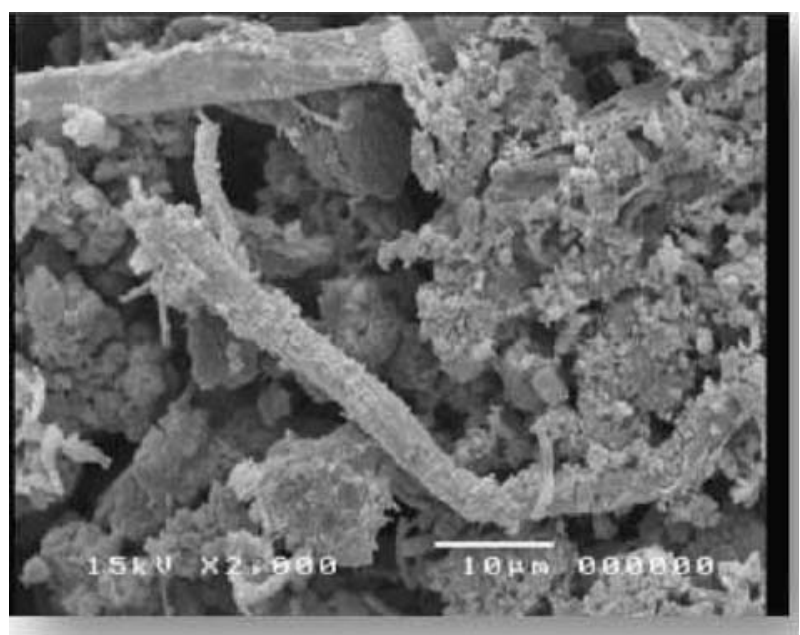

\section{The ITASEL Project: Italian Search for Extraterrestrial Life}

C.B. Cosmovici ${ }^{1}$, S. Montebugnoli ${ }^{2}$, M. Bartolini ${ }^{1}$, E. Flamini $^{3}$, S. Pluchino ${ }^{1}$, E. Salerno ${ }^{1}$, L. Zoni ${ }^{1}$

${ }^{1}$ IFSI-INAF; ${ }^{2}$ IRA-INAF; ${ }^{3}$ ASI

ITASEL is a Bioastronomy joint Project between IFSI (Istituto di Fisica dello Spazio Interplanetario) and IRA (Istituto di Radioastronomia) and financed by the Italian Space Agency (ASI).

Its main purpose is the development of new challenging spectral radio technologies to be applied to the Medicina (Bologna) and to other powerful radiotelescopes in order to detect water and life bearing molecules in comets and (exo) planetary systems.

After the promising discovery of the first water MASER emission in the solar system due to the catastrophic impact of Comet Shoemaker-Levy with the Jovian Atmosphere (1994), we decided to use this discovery as a powerful and unique diagnostic tool for water search in exoplanetary systems where cometary bombardments occur today as they occurred on our planet billions of years ago. Moreover calculations have shown that the $22 \mathrm{GHz}$ MASER emission can be observed also in water rich atmospheres where the necessary pumping can be delivered by photo-deposited energy which can affect the level populations.

Up to now we searched for water in 35 exoplanetary systems and we carried out observations of stellar regions where either cometary clouds have been discovered, or planetary systems have been indirectly detected and peculiar stars, such as red and brown dwarfs with strong IR-radiation.

Very faint possible transient signals have been tentatively identified in the last years and seem to be originated around five peculiar objects, but these observations need to be confirmed, using a recently developed multichannel spectrometer (SPECTRA-1).

The $22 \mathrm{GHz}$ MASER line was also detected for the first time in a comet (Hyakutake C/ 1996 ) and confirmed in Comet C/2002 V1-NEAT. Both comets were very close to the Sun ( 0.23 and 0.11 A.U. resp.) and the MASER excitation may have been originated by the strong coronal activity during the transit of the comets at perihelion. 
Recently the Cassini spacecraft has identified in the southern hemisphere of the Saturnian satellite Enceladus jets of ice particles carried by water vapour probably originated from liquid water sources below the satellite's surface.

Thus new observations are now carried out at Medicina in collaboration with the JIVE Institute (NL) in order to verify the possibility of detecting the MASER emission also from icy satellites in the solar system. A possible detection would be also very important for stating if a pumping model for the water molecules based on the magnetohydrodynamic interaction of a satellite or of the rings with the Saturnian magnetosphere could be taken into account.

SETI (Search for Extraterrestrial Intelligence)-observations are also carried out within the ITASEL project at Medicina (Bologna) using the $32 \mathrm{~m}$ dish and the Northern Cross, a large T-shaped parabolic/cylindrical antenna $\left(30,000 \mathrm{~m}^{2}\right)$. The automatic observations are carried out in "piggy back" mode using a SERENDIP IV high resolution spectrometer. An extremely powerful processing board based on a multi-FPGAs (Field Programmable Gate Array) core has been developed and is under programming.

E-mail: cosmo@ifsi-roma.inaf.it

\section{Analytical Developments for the Search of Enantiomeric Excess in Extraterrestrial Environment}

Grégoire Danger $^{1}$, David Ross ${ }^{2}$

${ }^{1}$ Institut D'Astrophysique Spatiale, Orsay, France; ${ }^{2}$ National Institute of Standards and Technology, Gaithersburg, USA

The search for signs of current or past life on Mars and elsewhere in the solar system is one of the most important and exciting objectives for many of the world's space agencies. Future missions are expected to send a rover to the surface of Mars with the capabilities to perform detailed, in situ chemical and biochemical analyses specifically aimed at the detection of extant or extinct life. Of the many potential biomarkers that could be targeted in a search for signs of life on other planets, amino acids are ideal candidates (Bada et al., 1997).

Amino acids are readily synthesized through abiotic (or prebiotic) processes, are abundant in the solar system, and, as has been demonstrated by life on Earth, can form biomacromolecules with highly varied biochemical functionality. Furthermore, most amino acids (as well as other biomolecules) are chiral, meaning that they occur in two enantiomeric forms that differ only in that they are nonsuperimposable mirror images of each other. Actually, abiotic processes seem to always produce amino acids in racemic mixtures - with equal concentrations of the two enantiomers. But in living organisms, because of the controlled structure required for the functioning of biomacromolecules, their components (e.g. amino acids) are expected to be found exclusively in one enantiomeric form. Thus, amino acids synthesized by current or past life would be readily distinguishable from those resulting from abiotic processes through an analysis of their chirality. Consequently, the discovery of non-racemic amino acids-with one enantiomer more prevalent than the other-would be considered to be a possible indication of past or present life (Bada et al., 1997).

For chiral analyses with low detection limit, integrated microfluidic lab-on-a-chip technologies offer many advantages that are particularly suited to the problem of in situ analysis including small size and weight, low power consumption, and capabilities for automation (Pumera, 2007). Furthermore, microfluidic CE devices with fluorescence detection such as the Mars Organic Analyzer (MOA) can provide detection limits as low as 0.5 parts per trillion (low nanomolar in solution) (Skelley et al., 2005). However, because no organic molecules have ever 
been detected on Mars, it is not clear what detection limit will be required. Consequently, it is important to improve the detection limits of such platforms as much as possible.

Temperature gradient focusing (TGF) (Ross et al., 2002) and Gradient Elution Isotachophoresis (GEITP) (Shackman et al., 2007) are recently described techniques that combine high resolution electrophoretic separation with built in concentration enhancement for low detection limits. Although TGF and GEITP have a number of advantages over conventional CE in terms of sensitivity, simplicity, and robustness, its primary advantage for application to biomarker detection may be its flexibility: With TGF and GEITP, the detection limit and the resolution can be easily improved without changing the device hardware but simply through modification of the operational parameters of the device (Munson et al., 2007; Danger et al., 2008a; Danger et al., 2008b). Furthermore, TGF and GEITP are performed with the same apparatus which provide analysis duplications on a same apparatus which limits cost, size and weight.

We present proof-of-concept experiments to examine the feasibility of TGF and GEITP for trace chiral amino acids analysis. Using a very low concentration of chiral selector, the chiral techniques provide a high resolution separation of a mixture of six to seven different amino acids (five chiral), with only few overlapping peaks

Bada, J. L., McDonald, G. D. (1997), extraterrestrial handedness? Science, 275: 942-943. Danger, G., Ross, D., (2008a), Chiral Separation with Gradient Elution Isotachophoresis for future in situ extraterrestrial analysis, Electrophoresis, Accepted.

Danger, G., Shackman, J., Ross, D., (2008b), Development of a Temperature Gradient Focusing Method for in situ Extraterrestrial Biomarker Analysis, Electrophoresis, Accepted. Munson, M., Danger, G., Shackman, J., Ross, D., (2007), Temperature Gradient Focusing with Field-Amplified Continuous Sample Injection for Dual-Stage Analyte Enrichment and Separation, Anal. Chem., 79:6201-6207.

Pumera, M. (2007), Microfluidics in amino acid analysis, Electrophoresis, 28:2113-2124. Ross, D., Locascio, L., (2002), Microfluidic temperature gradient focusing, Anal. Chem., $74: 2556-2564$.

Shackman, J., Ross, D., (2007), Capillary Gradient Elution Isotachophoresis for Enrichment and Separation of Biomolecules, Anal. Chem., 79:6641-6649.

Skelley, A. M., Scherer, J. R., Aubrey, A. D., Grover, W. H., Ivester, R. H. C., Ehrenfreund, P., Grunthaner, F. J., Bada, J. L., Mathies, R. A. (2005), Development and evaluation of a microdevice for amino acid biomarker detection and analysis on Mars, Proc. Natl. Acad. Sci. U. S. A., 102:1041-1046.

E-mail: dangergregoire@yahoo.fr

\section{Testing the Lithopanspermia Theory in the Foton-M3 Mission: Simulation of Interplanetary Transfer and Re-entry Process of Epi- and Endolithic Microbial Communities with the Lithopanspermia Experiment}

R. de la Torre ${ }^{1}$, L.G. Sancho ${ }^{2}$, G. Horneck ${ }^{3}$, P. Rettberg ${ }^{3}$, C. Ascaso ${ }^{4}$, A. de los Ríos ${ }^{4}$, J. Wierzchos ${ }^{5}$, J.P. de Vera ${ }^{6}$, S. Ott ${ }^{6}$, C. Cockell $^{7}$, K. Olsson ${ }^{7}$, J.M. Frías ${ }^{1}$, R. Demets ${ }^{8}$

${ }^{1}$ INTA (Spanish Aerospace Research Establishment); ${ }^{2}$ UCM (Univ. Complutense Madrid); ${ }^{3}$ DLR (German Aerospace Research Establishment); ${ }^{4}$ CSIC (Scientific Research Council); ${ }^{5} \mathrm{UL}$ (Univ. Lérida); ${ }^{6} \mathrm{HHU}$ (Heinrich-Heine Univ.); ${ }^{7} \mathrm{OU}$ (Open Univ.); ${ }^{8} \mathrm{ESA}$ (European Space Agency)

The objective of this experiment was to test experimentally the hypothesis of lithopanspermia, which supports interplanetary transfer of rock inhabiting life by means of meteorites: 
microorganisms have to survive (1) the impact ejection process from the planet of origin; (2) travelling through space; (3) capture and landing on another planet. In the experiment "Lithopanspermia" on board of the FOTON-M3 satellite (14.09.07) steps 2 and 3 of this scenario have been experimentally tested. We have selected as test systems for step 2 the bipolar epilithic lichen species Rhizocarpon geographicum and Xanthoria elegans on their natural rock substrate, as well as their fruiting bodies (reproduction structures), the endolithic microbial communities from the Atacama Desert with the cyanobacteria Chroococcidiopsis, the epilithic microbial communities from cliffs in the south-east of the UK with cyanobacterial akinetes of Anabaena, and the vagrant lichen species Aspicilia fruticulosa. Before exposure to outer real space conditions within the BIOPAN-6 facility of ESA, preparatory space simulation studies (UV solar spectrum radiation and vacuum $10^{-2} \mathrm{~Pa}$ ) were performed at the Spasolab-Laboratory of INTA (March-April 2007), to demonstrate the suitability of those lichen species.

After flight (10 days exposure to harsh space conditions in low Earth orbit at about $300 \mathrm{~km}$ altitude) and recovery, the survival capacity of the microbial communities has been assayed. First analyses have confirmed a fast recovery of the biological activity (chlorophyll a-fluorescence) of the lichen (epilithic and vagrant lichen), similar as the pre-flight activity, comparative to the high survival rates observed in the experiment Lichens onboard of the Foton-M2 mission (de la Torre et al. 2007; Sancho et al., 2007). First results of Confocal Scanning Laser Microscopy have shown maintenance of the vitality of epilithic samples..Ultrastructural changes are being analyzed by Transmission Electron Microscopy and cryoscanning. Furthermore, concerning the germination capacity of ascospores of Xanthoria elegans stimulation seems to have occurred. The epilithic cyanobacteria community did not survive the harsh conditions; however, the resting state cells of Anabaena did. Step 3 of Lithopanspermia has been tested with Rhizocarpon geographicum on its granite rock substrate, integrated in the thermal protection shield of the Foton capsule by use of the STONE facility, thereby simulating the external layer of a meteorite. The lichen did not survive this re-entry process. Mineralogical and petrologic studies have shown compositional and structural changes of the granite.

De la Torre et al. (2007). BIOPAN experiment LICHENS on the Foton-M2 mission: preflight verification tests of the Rhizocarpon geographicum-granite ecosystem, Adv. Space Res. 40, 1665-1671.

Horneck et al. (2008). Microbial rock inhabitants survive hypervelocity impacts on Mars-like host planets: First phase of Lithopanspermia experimentally tested, Astrobiology, 8: 17-29.

Sancho L. et al. (2007). Lichens survive in space. Astrobiology, 7: 443-454.

Stöffler D. et al. (2007). Experimental evidence for the potential impact ejection of viable microorganisms from Mars and Mars-like planets Icarus, 186: 585-588.

E-mail: torrenr@inta.es

\section{Evidence of Catalytic Activities from and Inside Meteorites. Did They Contribute to the Early Life by Increasing Molecular Complexity of a "Primitive Soup"?}

\section{Rosanna del Gaudio ${ }^{1}$, Bruno D’Argenio ${ }^{2}$, Giuseppe Geraci ${ }^{1}$}

${ }^{1}$ Dept of Biological Sciences, Section of Gen. and Mol. Biol.; ${ }^{2}$ Dept. of Earth Sciences, University of Naples Federico II, Via Mezzocannone 8, 80134 Napoli

The origin and dispersion of Life in the Universe is a long debated scientific and philosophical issue and, in this context, much work has been devoted to the analysis of different types of meteorites to reveal in them the signature or the remnants of possible forms of life. 
We have developed and applied an innovative approach (Geraci et al. 2007), aimed at revealing not life itself, or organic components, but the ability of meteorites to perform reactions operative in present-day life. To this aim we have carried out experiments on several fragments of iperstenic chondrites, looking for conditions permitting them to express catalytic activity. We found that, in suitable environments, components of the meteorite fragments are able to catalyze inorganic and organic reactions. Samples initially used were different specimens from two iperstenic chondrite swarms (Mòcs and Holbrook) fallen, respectively, in 1882 in Transylvania and in 1912 in the desert of Arizona, to minimize the possibility that the observed properties depended on the conservation conditions. Furthermore, similar results were obtained also using fragments of other iperstenic chondrites fallen on Earth in areas with different climates and in different years, each with a different background.

Particularly, we report here that fragments of iperstenic chondrite perform, in specific conditions (Geraci et al. 2007), glycosidase activity on $\alpha$ - and $\beta$-glycoside bonds and esterase activity both in water and in organic solvents. Those activities have been revealed also on substrates commonly employed in biomolecular laboratory analyses. In addition, meteorite fragments produce complex metal-organic structures whose material is endowed of physical and chemical properties not present in the starting meteorite sample, such as an amazing magnetism and ability to absorb light.

Those structures appear hollow, semi-transparent and pigmented orange-red, from pale to deep ruby. Their exterior is made of repetitive micro-nano units, having one side flat, laying on a thin organic layer, and the other brush-like. They appear only in aerobic conditions, indicating that redox reactions have a role in their autopoietic formation. Moreover, when damaged, they are capable to regenerate/repair themselves upon suitable external stimulation. Preliminary analytical results on the complexity of their organic and inorganic areas and on their repetitive polymeric structures demonstrate the ability of their growth processes to selectively accumulate and use externally provided biomolecules, some of which appear even chemically modified and in new molecular combinations.

The results so far obtained do not prove or exclude the possibility that those structures, having a complex chemistry, might be examples of proto-metabolic reactions occurred in a pre-biotic context. However, they are certainly the result of a number of coordinated activities and only some of them can be attributed to the meteorite components.

The data presented here lend support to the hypothesis that these "activities" might have participated to increase the molecular complexity of an initial "primitive soup" contributing to trigger the emergence of life.

Geraci G, D’Argenio B. del Gaudio R. (2007) Italian Patent RM2003A000026 granted, Patent pending EPO, USA.

E-mail: rosanna.delgaudio@unina.it

\section{Detecting Biosignatures of an Evolving Earth-Like Atmosphere via New Worlds Observer}

Julia DeMarines, Webster Cash, Giada Arney, Phil Oakley

University of Colorado

Over 200 extrasolar planets have been found in the last decade using indirect means, such as Doppler shift, and only one extrasolar planet has been directly imaged. New Worlds Observer is a mission that will revolutionize the direct detection of extrasolar planets by not only having the 
capability to image terrestrial-sized planets close to the star, but will also be able to analyze the spectrum of the planet's atmosphere and surface. We have simulated what an "Earth" will look like as a function of its atmospheric evolution. The biosignatures of the Earth are shown to evolve significantly and the current Earth is not the same as the younger Earth. We have found that the now-vanished signatures of a young life-bearing planet could be visible to the New Worlds Observer. NWO uses the visible band for detection of biosignatures like $\mathrm{O}_{2}$ (at $761 \mathrm{~nm}$ ) and $\mathrm{CH}_{4}$ (at $725 \mathrm{~nm}$ ). In our simulations we have been able to detect $\mathrm{O}_{2}$ at levels well below the current abundance and $\mathrm{CH}_{4}$ at levels well below those found on the younger Earth. This presents the possibility of detecting microbial life (methanogens) as early as 1.5 billion years after the formation of a planet, or photosynthetic life on a more mature planet.

Des Marais, D. J., et al. (2002). Remote Sensing of Planetary Properties and Biosignatures on Extrasolar Terrestrial Planets. Astrobiology. June 1, 2002, 2(2): 153-181.

Kaltenegger, L. et al. (2007). Spectral Evolution of an Earth-like Planet. The Astrophysical Journal, 658:598-616.

Kasting, J.F. Environmental constraints on the origin of life, Commentarii 4, N. 3, pp. 133147, Pontifical Academy of Sciences, Rome. Reprinted in: Encyclopedia Italiana (in press). Kasting, J.F. and L.L. (1988). Brown. Setting the stage: the early atmosphere as a source of biogenic compounds. In The Molecular Origins of Life: Assembling the Pieces of the Puzzle, A. Brack, ed., Cambridge Univ. Press, pp. 35-56.

Kasting, J. F., Siefert, J. L. (2002). Life and the Evolution of Earth's Atmosphere. Science, Vol. 296. no. 5570, pp. 1066-1068.

Mojzsis, S. J., et al. (1996). Evidence for life on Earth before 3,800 million years ago. Nature, 384, 55-59.

Schindler, T. L., Kasting, J. F. (2000). Spectra of Simulated Terrestrial Atmospheres Containing Possible Biomarker Gases. Icarus Volume 145, Issue 1, Pages 262-271.

E-mail: Julia.DeMarines@,colorado.edu

\section{ESA experiment BIOPAN-6-Germination and Growth Capacity of Lichen Symbiont Cells and Ascospores After Space Exposure}

J.P. de Vera ${ }^{1}$, S. Ott ${ }^{1}$, R. de la Torre ${ }^{2}$, L.G ${ }^{\text {a }}$ Sancho $^{3}$, G. Horneck ${ }^{4}$, P. Rettberg ${ }^{4}$, C. Ascaso ${ }^{5}$, A. de los Ríos ${ }^{5}$, J. Wierzchos ${ }^{6}$,C. Cockell ${ }^{7}$, K. Olsson ${ }^{7}$, J.M. Frías ${ }^{8}$, R. Demets ${ }^{9}$

${ }^{1}$ HHU (Heinrich-Heine-University); ${ }^{2}$ INTA (Spanish Aerospace Research Establishment); ${ }^{3}$ UCM (Univ. Complutense Madrid); ${ }^{4}$ DLR (German Aerospace Research Establishment); ${ }^{5} \mathrm{CSIC}$ (Scientific Research Council); ${ }^{6} \mathrm{UL}$ (Univ. Lérida); ${ }^{7} \mathrm{OU}$ (Open Univ.); ${ }^{8}$ INTA-CAB (Centro de Astrobiología); ${ }^{9}$ ESA (European Space Agency)

In the context of Lithopanspermia investigations have been performed to investigate the ability of different organisms to resist scenarios of the natural interplanetary transfer of life from a donor planet (host planet) to an acceptor planet. Whereas the main focus of previous studies was on the resistance of bacteria and their colony forming capacity after space exposure, only a few experiments on eukaryotic microorganisms and especially on symbiotic organization forms such as lichens, have been performed in space (de la Torre et. al. 2007, Sancho et al. 2007). These experiments have concentrated on photosynthesis analysis as a first step to understand the maintenance of physiologic activity of eukaryotic organisms after exposure to space conditions. As a next step, physiologic activity, reproduction capacity and "healthy" cell structures as essential parts of vitality check were analyzed after the last ESA experiment on BIOPAN-6 on 
Foton M3. Besides the examination of photosynthetic activity determination by use of chlorophyll a-fluorescence, CLSM analysis by the use of LIVE/DEAD staining dyes and culture experiments for verification of germination and growth capacity of both of the lichen symbionts (alga and fungi), were performed. In this case, new results are now clearly emphasizing quantitatively the high survival capacity and maintenance of germination and growth capacity of both of the investigated symbionts which form the epilithic lichen species of Rhizocarpon geographicum and Xanthoria elegans, although they were exposed to harsh space conditions with an exposure time of 10 days. About $80 \%$ to $90 \%$ of ascospores of both of the analyzed lichens were able to germinate and to grow into well-developed mycelia. In detail: results of germination and growth capacity analysis of ascospores of the lichen $X$. elegans have shown no damage on growth behavior, if compared to the control analysis, differing only in the starting point of germination, which is about 1 to 2 days earlier than under control conditions ( 1 to 2 days after sporulation instead of 2 to 4 days). Results of analysis on ascospores of $R$. geographicum indicate an important role of desiccation for successful germination. Without the vacuum treatment in space, the control samples were not able to germinate. This implicates the necessity of very dry conditions for the break down of ascosporic cell walls to foster the ability of germination, what can be expected also in natural habitats (high mountain regions). Analyses on the growth multiplication factor of photobiont cells (alga) are indicating a higher degree of space influence on $X$. elegans. Photobiont cells of $R$. geographicum maintained their doubling rate of about 12 days compared to control conditions, whereas the doubling rate of photobiont cells of $X$. elegans was seriously affected after space exposure, showing a severe retardation (control conditions: doubling rate $\left(d_{\mathrm{r}}\right)=$ 7 days, after space exposure: $d_{\mathrm{r}}=12$ days).

De la Torre et al. (2007). BIOPAN experiment LICHENS on the Foton-M2 mission: preflight verification tests of the Rhizocarpon geographicum-granite ecosystem, Adv. Space Res. 40, 1665-1671, doi:10.1016/jasr.2007.02.022.

Sancho L. et al. (2007). Lichens survive in space. Astrobiology, 7: 443-454.

E-mail: devera@uni-duesseldorf.de

\section{STONE 6 Experiment: An Investigation of the Survival of Microfossils During Atmospheric Entry}

Frédéric Foucher ${ }^{1}$, Frances Westall ${ }^{1}$, Jean-Michel Bény ${ }^{2}$, Franz Brandstätter ${ }^{3}$, René Demets ${ }^{4}$

${ }^{1}$ Centre de Biophysique Moléculaire, UPR CNRS 4301, Orléans, France; ${ }^{2}$ Institut des Sciences de la Terre d'Orléans, UMR CNRS 6113, Orléans, France; ${ }^{3}$ Naturhistorisches Museum, Burgring 7, 1010 Wien, Austria; ${ }^{4}$ European Space \& Technology Centre (ESTEC), Keplerlaan 1, Postbus 299, 2200 AG Noordwijk, The Netherlands

To date, the oldest traces of life occur in cherts (silicified volcanic silts and sands) that were deposited in coastal environments 3.5 billion years ago (Schopf et al., 2007; Brasier et al., 2004; Ueno et al., 2004; Westall et al., 2006; Westall and Sotham, 2006). These structures represent already relatively evolved organisms, including anaerobic photosynthesisers. This implies that life therefore had to have appeared much earlier (Westall and Southam, 2006). However, the study of older traces of life on Earth is limited by the lack of suitable material since plate tectonics has destroyed older crustal material and the few remaining enclaves of 3.8-4.0 Ga rocks are too heavily metamorphosed to provide useful information. On the other hand, ancient rocks on our planetary neighbour Mars from the Noachian period ( 4.5 to 3.5 billion years ago) could contain 
traces of fossil life dating back to the missing first billion years on Earth. One means of studying the Noachian rocks is to return suitable samples from Mars to Earth (Mars Sample Return mission 2020). Another field of investigation would be to analyse Martian sedimentary meteorites, possibly dating back to the Noachian period. To date, only basaltic martian meteorites have been discovered although there is evidence of abundant sedimentary rocks on Mars.

The STONE 6 experiment (September 2007, ESA) tested the survivability of Mars analogue sediments embedded in the heat shield of a FOTON capsule during entry into the Earth's atmosphere. One of the sediments used was a silicified volcanic sand from the 3.5 Ga-old "Kitty's Gap Chert", in the Pilbara region, NW Australia, deposited in a littoral environment. This rock is considered to be a good analogue for a lithified Noachian volcanic sediment. Moreover, it contains small colonies of fossilised prokaryote-like microbes (Westall et al., 2006). The first optical observation shows that a white fusion crust formed during entry, in contrast with the black crust of basaltic meteorites. Atomic Force Microscopy and Scanning Electron Microscopy were used to study the survival of the microfossils and Raman spectrometry for studying the evolution of the composition through the sample thickness. Even if the Raman spectrometry analysis shows the graphitization of the kerogenous material with increasing temperature gradient, we demonstrate that the microfossiliferous structures located deeper than $1.5 \mathrm{~cm}$ from the outer sample surface were well preserved. We conclude that if sedimentary Martian meteorites were found on Earth, they could contain eventual traces of extraterrestrial life.

Brasier, M., Green, O., Lindsay, J., and Steele, A. (2004), Earth's Oldest (3.5 Ga) Fossils and the 'Early Eden Hypothesis': Questioning the Evidence Origin of Life and Evolution of the Biosphere, 34:257-269.

Schopf, J. W., Kudryavtsev, A. B., Czaja, A. D., and Tripathi, A. B. (2007), Evidence of Archean life: Stromatolites and microfossils, Precambrian Research, 158:141-155.

Ueno, Y., Yoshioka, H., Maruyama, S., and Isozaki, Y. (2004), Carbon isotopes and petrography of kerogens in 3.5-Ga hydrothermal silica dikes in the North Pole area, Western Australia, Geochimica et Cosmochimica Acta, 68:573-589.

Westall, F., de Vries, S. T., Nijman, W., Rouchon, V., Orberger, B., Pearson, V., Watson, J., Verchovsky, A., Wright, I., Rouzaud, J. N., Marchesini, D., and Severine, A. (2006) The 3.466 Ga "Kitty's Gap Chert", an early Archean microbial ecosystem, Geological Society of America, Special Paper 405:105-131.

Westall, F. and Southam, G. (2006), The Early Record of Life Archean, Geodynamics and Environments, 164:283-304.

E-mail: frederic.foucher@cnrs-orleans.fr

\section{Experimental Silicification of Thermophilic Microorganisms. Relevance for Early Life on Earth and Mars}

F. Orange ${ }^{1,2}$, F. Westall ${ }^{1}$, J.-R. Disnar ${ }^{2}$, D. Prieur ${ }^{3}$, P. Gautret ${ }^{2}$, M. Le Romancer ${ }^{3}$, C. Dfarge ${ }^{2}$

${ }^{1}$ Centre de Biophysique Moléculaire, CNRS, Rue Charles Sadron, 45071 Orléans Cedex 02; ${ }^{2}$ Institut de Sciences de la Terre d'Orléans, 1A Rue de la Frollerie, 45071 Orlans Cedex 02; ${ }^{3}$ Université de Bretagne Occidentale, Institut Universitaire Europen de la Mer, Technople Brest-Iroise, 29280 Plouzan (France).

Since the earliest life forms known to date $(>3 \mathrm{Gyr})$ were preserved due to the precipitation of dissolved silica on cellular structures (silicification), we undertook an experiment to 
silicify several microbial species (the Archaea Methanocaldococcus jannaschii and Pyrococcus abyssi, and the Bacteria Chloroflexus aurantiacus and Geobacillus sp.), representative of anaerobic, thermophilic microorganisms that could have existed in the environmental conditions of early Earth and early Mars. This is the first time that Archaea have been used in a simulated fossilisation experiment and one of the very first fossilisations of thermophilic microorganisms. The experimental silicification was monitored by electron microscopy for a morphological study, and by chemical analysis (GC, GC-MS, HPLC) for a preliminary study of the preservation or degradation of the organic matter during silicification. This experiment demonstrated that not all microorganisms silicify under the same conditions. M. jannaschii cells lysed rapidly, although the EPS (extracellular polymeric substances) were preserved, as opposed to $P$. abyssi, Geobacillus sp. and C. aurantiacus where the cells were preserved and fossilized with differing degrees of silicification between species. The microorganisms apparently used active mechanisms to protect themselves temporarily from silicification, such as EPS production or silica repulsion. These results suggest that differences between species have a strong influence on the potential for different microorganisms to be preserved by fossilisation. This study provides valuable insight into the silicification and preservation processes of the kind of microorganisms that could have existed on the early Earth. Knowledge of these mechanisms can be helpful for the search and the identification of microfossils in both terrestrial and extraterrestrials rocks, and in particular Mars.

E-mail: orange@cnrs-orleans.fr

\section{On the Transfer of Meteorites (and Life?) from Earth to the GI 581 System}

Tetsuya Hara, Masanobu Shigeyasu, Kazuma Takagi, Daigo Kajiura

Deptment of Physics, Kyoto Sangyo University, Kyoto 603-8555, Japan

It is investigated the probability that the meteorites of Earth origin are transferred to the super-Earth planets in the Gl 581 system. We take the collisional ejection process of the Chicxulub crater event (Hildebrand et al. 1991) as Earth origin. If we assume the appropriate size of the meteorites $(<1 \mathrm{~cm}$ in diameter), the number of meteorites to reach the Gl 581 system could be much greater than one. We have followed the ejection and capture rates estimated by Melosh (2003) and the discussion by Wallis and Wickramasinghe (2004). We believe that the ejection rate estimated by Melosh as 15 rocks $(>10 \mathrm{~cm}$ diameter) each year from solar system seems to be too small. Although it is not certain that the microorganisms within the size $(<1 \mathrm{~cm})$ of meteorites are still viable for several Myr, Earth origin meteorites could be transferred to the G1 581 system. If it is viable, we should consider the possibility of meteorites exchange between stellar systems more seriously.

Recently it has been reported that the detection of the super-Earth planet in the Gl 581 system which resides at the warming edge of the habitable zone of the star (Udry et al. 2007). There has been established that rocks can be ejected from planetary surface by colliding asteroids and comets. The Chicxulub crater event 65 Myr ago provides evidence of the collisional ejection process. The meteorites size is estimated about $10 \mathrm{~km}$ in diameter. The concept that micro-organisms could be transported has begun to attract scientific attention. To estimate the transfer probability, we put parameters as following that $N_{0}$ rocks are ejected from the solar system, the distance to the nearby star is denoted by ' $s$ ', and the cross 
section of the rock capture by the star system is $\sigma$. Then the number of captured rocks is $N_{\text {impact }}=N_{0} \sigma /\left(4 \pi s^{2}\right)$.

When the Chicxulub meteorite collided to Earth, it could be estimated that almost the same amount mass could be ejected from Earth. Then it is assumed that the ejected mass from the solar system is $f_{1} \times f_{2} \times M$, where $M$ is the mass of the Chicxulub meteorite. The factor $f_{1}(0.3)$ denotes the fraction of the mass ejected from Earth and $f_{2}(0.3)$ denotes the fraction of the mass ejected from the solar system. Taking that the mean diameter of rocks is $r(1 \mathrm{~cm})$ and the estimated diameter of the Chicxulub meteorite is $R(10 \mathrm{~km})$, the number of ejected rocks from solar system is $N_{0} f_{1} f_{2}(R / r)^{3} 10^{17}$. The distance to the Gl 581 is 20 light years so we take the representative value for $s\left(10^{20} \mathrm{~cm}\right)$. The problem is the cross section $\sigma$. If the effects of gravitational interaction such as swing by, the cross section could be enlarged to $\sigma f^{3}(1 \mathrm{AU})^{2}$ (Melosh, 2003) with the factor $f^{3}(0.1)$. Then the rough estimation is $N_{\text {impact }}=f_{1} f_{2} f_{3}(R / r)^{3}(1 \mathrm{AU})^{2} /\left(4 \pi s^{2}\right) 10^{2}$.

If we take the mean velocity of meteorites in the interstellar space as $10(\mathrm{~km} / \mathrm{s})$, the elapsed time to travel 20 lyr is several Myr.

Even though there are many uncertain factors, the probability of rocks originate from Earth to reach nearby star system is not so small. If the micro-organisms within the size $(<1 \mathrm{~cm})$ of meteorites are still viable for several Myr, we should investigate the panspermia theories much further.

Hildebrand, A et al. (1991). Chicxulub Crater; a possible Cretaceous/Tertiary boundary impact crater on the Yucatan Peninsula, Mexico. Geology, 19: 867-871.

Melosh, H. (2003). Exchange of Meteorites (and Life?) Between Stellar Systems. Astrobiology, 3:207.

Udry, S. et al. (2007). The HARPS search for southern extra-solar planets. (astro-ph/0704.3841) Wallis, M. and Wickramasinghe, N (2004). Interstellar transfer of planetary microbiota. MNRAS. 348:52.

E-mail: hara@cc.kyoto-su.ac.jp

\section{Lithopanspermia Revisited: Origin of Life on Ceres?}

Joop M. Houtkooper

Institute for Psychobiology and Behavioral Medicine, Justus-Liebig-Univerity, Giessen, Germany

After life gained a foothold on Earth, it is assumed it spread rapidly over all niches where conditions were suitable for originating life, so that the origin likely occurred only once. But did it occur on Earth? As Earth was sterilized during the LHB, about 700 My after the formation of the solar system, seeding by lithopanspermia is a definite possibility (Horneck et al., 2008). If so, the question is what the place of origin could be in the solar system. Possible sources of life for lithopanspermia include Earth itself (before LHB), Mars, Venus (if it had a more benign climate than today) and the icy bodies in the outer solar system. The mechanics of lithopanspermia entail the problems of ejection, preservation during transfer and arrival. The ejection of pieces of the surface into space requires achieving at least the escape velocity of the parent body. Preservation during travel from the parent body to the seeded "child body" appears to be a lesser problem. The arrival of spore-bearing 
meteorites is a more severe problem for airless bodies like the moon, because of the shock upon arrival, than for Earth where meteorites may survive through aerobraking. If we disregard the far-out bodies like Charon, and moons deep in the gravitational well of their planet like Europa, a likely parent body which remains is Ceres, which has had, or still has, an ocean more than $100 \mathrm{~km}$ deep, with hydrothermal activity at its rocky core (CastilloRogez et al., 2007). There, life may have originated early in the history of the solar system. Moreover, in this deep ocean it may well have survived the LHB. Therefore, Ceres' low escape velocity of $510 \mathrm{~m} / \mathrm{s}$ makes Ceres a likely parent body for life in the solar system. Can this hypothesis of Ceres as the parent body, also for life on Earth, be tested? The surface temperature of Ceres is in spots as high as $239 \mathrm{~K}$, sufficient for life in brine-filled channels in its dirty-ice crust to survive until today. Such life might employ photosynthesis or the compounds such as oxidants created by radiation for energy and possibly hydrogen peroxide as an antifreeze (Houtkooper and Schulze-Makuch, 2007). The detection of life in the surface layers of Ceres would support the hypothesis. Secondly, a commonality of Cerean life with Terran and possible Martian life would be expected. Third, biomarkers of Cerean life might be found in the ices at the Moon's poles and on the surface of other main belt asteroids, as there the arrival of chunks of Ceres' crust may have taken place at low velocity. The Dawn mission and future exploration of the Moon's polar regions may shed more light on this.

Castillo-Rogez, J.C., McCord, T.B., and Davies, A.G. (2007). Ceres: Evolution and present state. Lunar and Planetary Science XXXVIII: 2006-2007. Lunar Plan. Sci. Conf.

Horneck, G., Stffler, D., Ott, S., Hornemann, U., Cockell, C.S., Moeller, R., Meyer, C., de Vera, J.-P., Fritz, J., Schade, S., and Artemieva, N.A. (2008) Microbial Rock Inhabitants Survive Hypervelocity Impacts on Mars-Like Host Planets: First Phase of Lithopanspermia Experimentally Tested. Astrobiology 8: 17-44.

Houtkooper, J.M., and Schulze-Makuch, D. (2007) A possible biogenic origin for hydrogen peroxide on Mars: the Viking results reinterpreted. International Journal of Astrobiology 6: 147-152.

E-mail: joophoutkooper@gmail.com

\title{
Cryopreservation and Stability of Microbial Population in Permafrost
}

\author{
E.S. Karaevskaya ${ }^{1}$, E.A. Vorobyova ${ }^{1}$, G.A. Osipov $^{2}$, M.A. Petrova ${ }^{3}$
}

${ }^{1}$ Department of Soil Science, Lomonosov Moscow State University, Moscow; 119991, Russian Federation, Moscow GSP-1, Lomonosov Moscow State University, Leninskie Gory 1-12; ${ }^{2}$ Bakulev's Scientific Center of Cardiovascular Surgery of the Russian Academy of Medical Sciences 121552, Russian Federation, Moscow, Rublevskoye shosse, 135, Bakulev's Scientific Center of Cardiovascular Surgery RAMS; ${ }^{3}$ Institute of Molecular Genetics, Russian Academy of Sciences, Moscow, 123182 Russia

The diversity of cells and microbial activities both were studied to indicate life in Antarctic permafrost sediments, ground ices and ice sheet disposed from the day surface up to $5 \mathrm{~m}$ (Dry Valleys, the age up to 40,000 years). The strategy for bacterial survival under freezing in permafrost must include special mechanisms for adaptation to long-term freezing in nature. 
To investigate the viability of microbial populations, the following methods were used: evaluation of bacterial biomass in situ, investigation of kinetics of bacterial populations after thawing and numbers of culturable cells (CFU) on nutrient media, study of bacterial preliminary antibiotic resistance (to chloramphenicol, tetracycline, carbenicilline, gentamycin, kanamycin, streptomycin) and bacterial thermal limits of growth and osmotic stress. High resolution microscopy (SEM, AFM), epifluorescence microscopy, lipid biomarkers' analysis, 16sRNA analysis of isolated strains and routine microbiological techniques were applied. Living prokaryotic and eukaryotic microorganisms were observed in all samples investigated. The total cell's amount in Antarctic and Arctic samples ranged to $10^{7}-10^{8}$ cells per gram dry weight and for most of them significantly exceeded CFU number $\left(10^{2}-10^{6}\right)$. Among isolated strains from Antarctic permafrost were the representatives of gram positive bacteria Bacillus, Rhodococcus and gram negative bacteria Aureobacterium (Curtobacterium), or Comamonas (Aquaspirillum). For ancient Arctic ground ice among the dominants were gram positive strains of genera Arthrobacter, Promicromonospora and strains of gram negative bacteria of genera Flavobacterium. All isolated strains revealed the possibility to growth at wide range of temperatures. More than half of isolated bacterial strains were resistant to various antibiotics. Study of antibiotic resistance spectrum of all isolated from Arctic and Antarctic sediments strains showed not only single resistance to certain antibiotic, but also double resistance to various antibiotics. As revealed by method of 16sRNA analysis, among these strains were bacteria of genera Acinetobacter, Paenibacillus and Brevundimonas

It was revealed that endogenic physiological transformations of bacterial cells in permafrost sediments doesn't depend on the lithogenesis, but to a grater extent on long persistence of temperature/or water availability. It could be expected, that in conditions of prolonged cell multiplication braking, the adaptive mutations proceed in microbial cells, increasing the vitally important potential of microorganisms.

The obtained results provide new arguments to the whys and wherefores of the astrobiology search of life on other planets with dominated subzero temperatures (Mars).

E-mail: second_ks@mail.ru

\section{Pyrolysis GC/MS Technique Application to Exobiology}

\section{Yeghis Keheyan}

ISMN-CNR, c/o Dept. of Chemistry, University "La Sapienza", p.le Aldo Moro 5, Rome0185 , Italy

Many extraterrestrial objects are known to contain organic mater in the form of complex macromolecular materials. Pyrolysis coupled with gas chromatography and mass spectrometry (Py-GC-MS) is known to be powerful tool in analysing such materials and has been applied to the study of different complex organic matter contained in meteorites and interplanetary dust particles. The results of pyrolysis experiments to estimate survivability of different compounds of exobiological interest in oxygen-free ( $\mathrm{He})$ atmosphere will be reported.

E-mail: yeghis.keheyan@uniroma1.it 


\section{Early Survival, Pigment Spectra, and Productivity of Photosynthesis on M Star Planets}

Nancy Y. Kiang ${ }^{1,10}$, Antígona Segura ${ }^{2,10}$, Giovanna Tinetti ${ }^{3,10}$, Govindjee ${ }^{4}$, Robert E. Blankenship $^{5}$, Martin Cohen ${ }^{6,10}$, Janet Siefert ${ }^{7,10}$, David Crisp ${ }^{8,10}$, Victoria S. Meadows ${ }^{9,10}$

${ }^{1}$ NASA Goddard Institute for Space Studies, U.S.A.; ${ }^{2}$ Instituto de Ciencias Nucleares, Universidad Nacional Autónoma de México; ${ }^{3}$ Dept. of Physics and Astronomy, STFC/ University College London, Great Britain; ${ }^{4}$ Departments of Plant Biology and Biochemistry, University of Illinois at Urbana-Champaign, U.S.A.; ${ }^{5}$ Department of Biology and Chemistry, Washington University, U.S.A.; ${ }^{6}$ Radio Astronomy Laboratory, University of California, Berkeley, U.S.A.; ${ }^{7}$ Department of Statistics, Rice University, U.S.A.; ${ }^{8}$ NASA Jet Propulsion Laboratory, California Institute of Technology, U.S.A.; ${ }^{9}$ Department of Astronomy, University of Washington, Seattle, USA; ${ }^{10}$ NASA Astrobiology Institute

M stars are the most abundant type of star in our galaxy, but, on an Earth-like planet in the habitable zone of an M star, could photosynthetic life could develop given the damaging UV flares of young, active M stars? If so, could it thrive, given the low amount of visible light emitted relative to infared? If photosynthesis in the near-infrared were to dominate, could it be productive enough to create detectable biosignatures, and would atmospheric oxygen be feasible? At what wavelength will photosynthetic reaction centers on $\mathrm{M}$ star planet most likely operate? In Kiang, et al. (2007a), we looked at Earth's example of the adaptation of land plants to the Solar spectrum and identified rules for how pigment light harvesting favors the "red edge" of Earth vegetation. Then in Kiang, et al. (2007b), we took planetary atmospheric compositions simulated by Segura, et al. $(2003,2005)$ for Earth-like planets around modeled M1V and M5V stars, and around the active M4.5V star AD Leo, with scenarios using Earth's atmospheric composition as well as very low $\mathrm{O}_{2}$ content, in case anoxygenic photosynthesis dominates. With a line-by-line radiative transfer model we calculated the incident spectral photon flux densities at the surface of the planet and under water. We identified bands of available photosynthetically relevant radiation, and found that photosynthetic pigments on planets around $M$ stars may peak in absorbance in the NIR, in bands at $0.93-1.1,1.1-1.4,1.5-1.8$, and $1.8-2.5 \mu \mathrm{m}$. However, underwater organisms will be restricted to wavelengths shorter than $1.4 \mu \mathrm{m}$ and more likely below $1.1 \mu \mathrm{m}$. M star planets without oxygenic photosynthesis will have photon fluxes above $1.6 \mu \mathrm{m}$ curtailed by methane. Longer-wavelength, multi-photosystem series would reduce the quantum yield but could allow for oxygenic photosystems at longer wavelengths, restricted to below possibly $1.1 \mu \mathrm{m}$. M star planets could be a half to a tenth as productive as Earth in the visible, but exceed Earth if useful photons extend to $1.1 \mu \mathrm{m}$ for anoxygenic photosynthesis. Under water, organisms would still be able to survive UV flares from young $M$ stars and acquire adequate light for growth.

Kiang, N.Y., J. Siefert, Govindjee, and R.E. Blankenship. (2007a). "Spectral signatures of photosynthesis I: Review of Earth organisms," Astrobiology, Special Issue on M Stars, 7(1): 222-251.

Kiang, N.Y., A. Segura, G. Tinetti, Govindjee, R.E. Blankenship, M. Cohen, J. Siefert, D. Crisp, and V.S. Meadows. (2007b). "Spectral signatures of photosynthesis II: coevolution with other stars and the atmosphere on extrasolar worlds," Astrobiology, Special Issue on M Stars, 7(1): 252-274. 
Segura, A., J. F. Kasting, V. Meadows, M. Cohen, J. Scalo, D. Crisp, R. A. H. Butler and G. Tinetti (2005). "Biosignatures from Earth-like planets around M dwarfs." Astrobiology 5 (6): 706-725.

Segura, A., K. Krelove, J. F. Kasting, D. Sommerlatt, V. Meadows, D. Crisp, M. Cohen and E. Mlawer (2003). "Ozone concentrations and ultraviolet fluxes on Earth-like planets around other stars." Astrobio 3: 689-708.

E-mail: nkiang@giss.nasa.gov

\section{Amino Acid Precursors Formed in Upper and Lower Titan Atmosphere and Their Relevance to Origins of Life}

Toshinori Taniuchi ${ }^{1}$, Tomohiro Hosogai ${ }^{1}$, Takeo Kaneko ${ }^{1}$, Bishun N. Khare ${ }^{2}$, Christopher P. $\mathrm{McKay}^{2}$, Kensei Kobayashi ${ }^{1}$

\section{${ }^{1}$ Yokohama National University; ${ }^{2}$ NASA Ames Research Center}

Titan, the largest moon of Saturn, has dense (ca. 1,500 Torr) atmosphere mainly composed with nitrogen and methane. The upper atmosphere of Titan has organic aerosol, so that it is difficult to observe the lower atmosphere and surface of Titan. There have been a large number of experiments simulating the action of solar UV and Saturn magnetosphere electrons in Titan upper atmosphere. The solid products formed in such experiments were sometimes called tholins. On the other hand, major energy in the lower atmosphere would be cosmic rays. We performed experiments simulating the lower atmosphere of Titan by irradiation with high-energy protons. The irradiation products (the lower tholins) were compared with the products formed by plasma discharge (the upper tholins).

Mixtures of methane (1-10\%) and nitrogen (balance; total pressure was 700 Torr) sealed in glass tubes were irradiated with $3 \mathrm{MeV}$ protons from a van de Graaff accelerator (Tokyo Institute of Technology). One Torr of the same kinds of mixture were subjected to plasma discharge in NASA Ames Research Center. Both products were analyzed by such techniques as FT-IR, GPC and Pyrolysis (Py)-GC/MS. Amino acids were identified and determined by HPLC, GC/MS and MALDI-TOF-MS.

Complex organic compounds (tholins) were formed in both proton irradiation (PI) and plasma discharge (PD). Molecular weight of PD-tholins estimated by GPC was a few thousands, and that of PI-tholins was several hundreds. Py-GC/MS gave a wide variety compounds including polyaromatic hydrocarbons and heterocyclic compounds in both tholins.

Hydrolysis of both tholins gave a wide variety of amino acids, and glycine was predominant. Energy yield (G-value) of glycine by PI (5\% methane) was 0.03 , which was much higher than that by PD (0.00009 in the case of $10 \%$ methane). It was estimated that the lower atmosphere $(0-150 \mathrm{~km}$ high) could be giving glycine precursors 200 times more than the upper atmosphere (425-825 km high) in Titan. Such complex amino acid precursors might be collected on the surface of Titan with rain of methane.

We can expect the same kind of chemical reactions in the primitive Earth. The composition of terrestrial primitive atmosphere is not known, but nitrogen should have been one of the major constituents together with methane or carbon monoxide as minor constituents. In such a case, formation of complex amino acid precursors (terra-tholins?) was possible (Kobayashi et al., 2001). It would be of great interest to detect complex amino acid precursors in the bottom of dried pond of Titan in the next Titan mission ("Tandem"?), which can help us to construct chemical evolution scenario of not only Titan but also primitive Earth. 
K. Kobayashi, H. Masuda, K. Ushio, A. Ohashi, H. Yamanashi, T. Kaneko, J. Takahashi, T. Hosokawa, H. Hashimoto and T. Saito (2001). Formation of bioorganic compounds in simulated planetary atmospheres by high energy particles or photons. Adv. Space Res., 27:207-215.

E-mail: kkensei@ynu.ac.jp

\section{Search for Extant Life in Extreme Environments by Measuring Enzymatic Activities}

Shuji Sato ${ }^{1}$, Kenta Fujisaki ${ }^{1}$, Kazuki Naganawa ${ }^{1}$, Takeo Kaneko ${ }^{1}$, Yuki Ito ${ }^{1}$, Yoshitaka Yoshimura $^{2}$, Yoshinori Takano ${ }^{3}$, Mari Ogawa ${ }^{4}$, Yukishige Kawasaki ${ }^{5}$, Takeshi Saito ${ }^{5}$, Kensei Kobayashi ${ }^{1}$

${ }^{1}$ Yokohama National University; ${ }^{2}$ Tamagawa University; ${ }^{3}$ Japan Agency for Marine-Earth Science and Technology; ${ }^{4}$ Yasuda Women's University; ${ }^{5}$ Institure of Advanced Studies

It has been recognized that terrestrial biosphere expands to such extreme environments as deep subsurface lithosphere, high temperature hot springs and stratosphere, and possible life in extraterrestrial life in Mars and Europa is discussed. It is difficult to detect unknown microorganisms by conventional methods like cultivation methods. Thus techniques to detect life in such environments are now required.

Enzymes are essential biomolecules that catalyze biochemical reactions. They can be detected with high sensitivity since one enzyme reacts with many substrate molecules to form many products. We tried to detect and characterize enzymes in extreme environments in surface soils in Antarctica and rocks in hydrothermal systems. Targeted enzymes are phosphatases, since they have low specificity and are essential for all the terrestrial organisms. Concentration and $\mathrm{D} / \mathrm{L}$ ratio of amino acids were also determined.

Core samples and chimney samples were collected at the Suiyo Seamount, Izu-Bonin Arc, the Pacific Ocean in 2001 and 2002, and in South Mariana hydrothermal systems, the Pacific Ocean in 2003, both in a part of the Archaean Park Project. Surface soil samples are obtained at the Sites 1-8 near Showa Base in Antarctica during the 47th Japan Antarctic exploration mission in 2005-6 and 2007-8.

Alkaline (or acid) Phosphatase activity in solid samples was measured spectrometrically by using $25 \mathrm{mM}$ p-nitrophenyl phosphate $(\mathrm{pH} 8.0$ (or $\mathrm{pH} 6.5)$ ) as a substrate. Phosphatase activities in extracts were measured fluorometrically by using 4-methylumberyferryl phosphate as a substrate. Concentration of amino acids and their enantiomeric ratios were also determined by HPLC and GC/MS.

Significant enzymatic activities were detected in both some of the hydrothermal sub-vent systems, chimney rocks and Antarctica soils, which is crucial evidence of the presence of vigorous microbial activities. It is consistent with the fact that large enantiomeric excess of L-form amino acids were found in the same core sequences. Chimney phosphatases showed optimum at higher temperature than E-coli phosphatase, while Antarctica phosphatases showed maximum activities at lower temperature.

In order to detect individual microorganisms, fluorescence microscopy technique was applied. It was proved that most of terrestrial microorganisms could be detected when we dyed soil samples with CFDA-AM, a substrate of esterases. We are developing a portable fluorescence microscope for in situ detection of extant organisms in the field.

We express our thanks to members of Archaean Park Project for the samples of hydrothermal systems. We also thank Dr. Manamu Fukui, Hokkaido University and the members of the 47th and 49th Japan Antarctic exploration missions.

E-mail: kkensei@ynu.ac.jp 


\title{
Organic Molecules in Class I Protoplanetary Disk
}

\author{
Yi-Jehng Kuan ${ }^{1,2}$, Yo-Ling Chuang ${ }^{1}$, Chian-Chou Chen ${ }^{1}$, Kuo-Song Wang ${ }^{2}$, Hui-Chun \\ Huang ${ }^{1}$
}

${ }^{1}$ Department of Earth Sciences, National Taiwan Normal University, Taipei, 116, Taiwan;

${ }^{2}$ Institute of Astronomy and Astrophysics, Academia Sinica, Taipei, 106, Taiwan

A number of Class 0 sources have been found to be rich in organic molecules, which are present in hot corinos. Since most of the material accreted during the Class 0 phase is consumed by the forming protostar, a meaningful comparison between interstellar, nebular and comet chemistries can only be made by studying the composition of the envelopes and disks of Class I sources. Recently Spitzer has surveyed more than 100 Class I and II YSOs and only detected hot organic molecules in IRS 46, a Class I source.

We have thus used the Submillimeter Telescope (SMT) to observe IRS 46 and we have detected $\mathrm{H} 2 \mathrm{CO}$ and $\mathrm{CH} 3 \mathrm{OH}$ toward IRS 46. The successful detection of these two organic molecules indicates recent icy mantle evaporation, hence the presence of an organically rich hot corino environment. Further high angular-resolution observations with the Submillimeter Array (SMA) will not only allow us to determine the organic inventory of IRS 46 but also enable us to compare the chemistry of nominal Class I hot corinos with those at the Class 0 phase. Some of the preliminary results from our SMT and SMA observations will be presented.

E-mail: kuan@ntnu.edu.tw

\section{Hydrothermal Alternation of Abiotically-Formed Complex Organic Compounds in Simulated Submarine Hydrothermal Environments}

\author{
Hironari Kurihara ${ }^{1}$, Taishi Ueki ${ }^{1}$, Takeo Kaneko ${ }^{1}$, Yoshinori Takano ${ }^{2}$, Kensei Kobayashi ${ }^{1}$ \\ ${ }^{1}$ Gradiate School of Engineering, Yokohama National University; ${ }^{2}$ Japan Agency for \\ Marine-Earth Science and Technology
}

Submarine hydrothermal systems (SHSs) has been considered possible sites of chemical evolution and generation of life on the Earth since their discovery in late 1970s. A number of experiments simulating the conditions of SHSs were conducted, and abiotic production and polymerization of amino acids were reported. On the other side, it was claimed that organic compounds, particularly amino acids, are not stable in such high temperature environments as SHSs.

In our early studies, not free amino acids but complex amino acids precursors with large molecular weights were formed abiotically from simulated primitive Earth atmosphere (a mixture of $\mathrm{CO}, \mathrm{N}_{2}$ and $\mathrm{H}_{2} \mathrm{O}$ ) (Takano et al., 2004). Such complex organics (hereafter referred as to $\mathrm{CNW}$ ) should have been delivered to SHSs in primitive ocean, where they were subjected to further alteration. We examined possible alteration of the complex organics in high-temperature high-pressure environments by the supercritical water flow reactor (SCWFR) (Islam et al. 2003) and an autoclave.

The complex amino acid precursors (CNW) were much stabler than free amino acids. While grainy structures of ca. $10 \mathrm{~nm}$ size were observed in CNW with a Transmission Electron Microscope (TEM), fused film-like structures of micrometer order size were formed after CNW was heated at $573 \mathrm{~K}$ for 2 min by SCWFR. It was possible that complex 
organic compounds delivered to primordial SHSs altered chemically and morphologically toward the generation of the first life.

Islam, Md. N., Kaneko, T., and Kobayashi, K (2003). Reactions of Amino Acids with a Newly Constructed[3000]Supercritical Water Flow Reactor Simulating Submarine Hydrothermal Systems. Bull. Chem. Soc. Jpn., 76, 1171

Takano, Y., Marumo, K., Yabashi, S., Kaneko, T., and Kobayashi, K., (2004). Curie-Point Pyrolysis of Complex Organics Simulated by Cosmic Rays Irradiation of Simple Inorganic Gas Mixture. Appl Pyys. Lett, 85, 1633

E-mail: d06sa503@ynu.ac.jp

\section{Pyrite as a Template for Carbon Fixation}

Paula Lindgren ${ }^{1}$, John Parnell ${ }^{2}$, Nils G. Holm ${ }^{1}$

${ }^{1}$ Department of Geology and Geochemistry, Stockholm University, Sweden; ${ }^{2}$ Department of Geology and Petroleum Geology, University of Aberdeen, UK

An important process in the evolution of life is the precipitation and concentration of organic species. There are several examples of minerals acting as templates for the accumulation and concentration of organic matter. These include for instance clays (e.g. Cairns-Smith and Hartman, 1986), radioactive minerals (e.g. Rasmussen, et al. 1993), zeolites and feldspars (e.g. Smith, et al. 1999) and the sulphide mineral pyrite $\left(\mathrm{FeS}_{2}\right)$ (e.g. Wächtershäuser, 1988). Wächtershäuser (1988) suggested that prebiotic chemistry and eventually life itself could have started on the surface of pyrite. Pyrite carries a slight positive electrical charge, and can attract negatively charged organic molecules, bringing them close enough to interact. Pyrite is also oil-wet in some circumstances (Yusupova, 2002). This means that if the mineral is exposed to a mix of oil and water, the oil will preferentially adhere to the surface of pyrite.

We have studied migrated organic matter in the Irish Carboniferous, including in sulphide deposits, to assess whether sulphides in fact do act as templates for organics. Here, pyrite was found acting as a template for carbon fixation in hydrothermal calcite veins, cutting through limestone. The pyrite crystals are ca. $1 \mathrm{~mm}$ in diameter and scattered throughout the vein matrix. The organic matter is migrated bitumen, and appears as smooth and rounded solid droplets, concentrated around the pyrite crystals. Scanning electron microscope analyses show the organics occurring as a ca. $150 \mu \mathrm{m}$ thick and even coating around the pyrite crystals.

Sulphide templates could be important for carbon fixation on Mars. There is widespread evidence of that sulphur species are prominent in Martian surface environments, assumed to have been introduced to the surface through volcanic activity. Currently, the Martian surface is highly oxidizing and therefore sulphates predominate, but early in the planet's history reducing conditions pertained. Accordingly it has been suggested that sulphides occurs on Mars (Burns and Fisher, 1990), now preserved at depth. Sulphides are also known to be present on Mars from Martian meteorites (e.g. Greenwood, et al. 2000). Sulphides are sources of fuel for micro-organisms that oxidize sulphides on Earth, and the same could have been the case on Mars (Bishop, et al. 2004).

The carbon coated pyrite in this study, is one example from the geological record showing that terrestrial sulphides can have a high potential for the preservation of organic materials. This could also be possible on Mars, and therefore Martian sulphides are good targets for seeking evidence of putative Martian life. 
Bishop, J.L., Dyar, M.D., Lane, M.D., and Banfield, J.F. (2004). Spectral identification of hydrated sulfates on Mars and comparison with acidic environments on Earth. International Journal of Astrobiology, 3: 275-285.

Burns, R.G. and Fisher, D.S. (1990). Evolution of sulphide mineralization on Mars. Journal of Geophysical Research, 95: 14169-14173.

Cairns-Smith, A.G. and Hartman, H. editors (1986). Clay minerals and the origin of life. Cambridge University Press, Cambridge.

Greenwood, J.P., Riciputi, L.R., McSween, H.Y., and Taylor, L.A. (2000). Modified sulfur isotopic compositions of sulfides in the nakhlites and Chasigny. Geochimica et Cosmochimica Acta, 64: 1121-1131.

Rasmussen, B., Glover, J.E., and Foster, C.B. (1993). Polymerisation of hydrocarbons by radioactive minerals in sedimentary rocks: Diagenetic and Economic Significance. Society for Geology applied to Mineral deposits, Special Publications, 9: 490-509.

Smith, J.V., Arnold, F.P., Parsons, I., and Lee, M.R. (1999). Biochemical evolution III: Polymerization on organophilic silica-rich surfaces, crystal-chemical modeling, formation of first cells, and geological clues. Proceedings of the National Academy of Sciences USA, 96: 3479-3485.

Wächtershäuser, G. (1988). Pyrite formation, the first energy source for life: a hypothesis. Systematic and Applied Microbiology, 10: 207-210.

Yusupova, T.N., Romanova, U.G., Gorbachuk, V.V., Muslimov, R.Kh., and Romanov, G.V. (2002). Estimation of the adsorption capacity of oil-bearing rocks: A method and its prospects. Journal of petroleum Science and Engineering, 33: 173-183.

E-mail: paula.lindgren@geo.su.se

\section{TANPOPO: Astrobiology Exposure and Micrometeoroid Capture Experiments on the KIBO, ISS}

Hajime Mita ${ }^{1}$, Akihiko Yamagishi ${ }^{2}$, Hajime Yano ${ }^{3}$, Kyoko Okudaira ${ }^{3}$, Kensei Kobayashi ${ }^{4}$, Shin-ichi Yokobori ${ }^{2}$, Makoto Tabata ${ }^{5}$, Hideyuki Kawai ${ }^{5}$, Hirofumi Hashimoto ${ }^{3}$, TANPOPO WG

${ }^{1}$ Fukuoka Institute of Technology; ${ }^{2}$ Tokyo University of Pharmacy and Life Sciences; ${ }^{3}$ Japan Aerospace Exploration Agency; ${ }^{4}$ Yokohama National University; ${ }^{5}$ Chiba University

TANPOPO, dandelion is an astrobiological mission, aiming to evaluate the possibility of interplanetary migration of microbes, organic compounds carried by micrometeoroid, onboard the Exposed Facility of the Japanese Experiment Module (JEM) 'KIBO' attached to the International Space Station (ISS) (Yamagishi et al., in press).

There has been a hypothesis to explain the early initiation of life on Earth, called "panspermia" (Arrhenius, 1908, Crick, 1981). According to this hypothesis, life has migrated to Earth from extra terrestrial objects. If it was possible, the reverse panspermia might occur from life-rich Earth as well. The finding of microfossil-like structure in a meteorite originated from Mars recalled this probability. Terrestrial living organisms on the Earth may have possibility to be ejected into outerspace by volcanic eruption or meteorite impact. We confirmed the presence of microbes at high altitude in atmosphere by sampling made by aircrafts and balloons (Yang, in press). The microbe-sampling experiments could be extended to the height of lower Earth orbit by using the ISS. It is also important to test if the microbe ejected from the Earth may survive under harsh space environment during their voyage to other planets. We will also conduct the survival test of microbes on the ISS. 
Another important subject on the origin of life is related to the pre-biotic production of organic compounds other than on Earth. The extra-terrestrial and outer-solar area might be the probable site for the pre-biotic organic compound synthesis. To test this hypothesis, simulation has been conducted on ground. We may obtain direct evidence by the intact meteoroid capture experiment planned by Tanpopo. It is also important to know what kind and degree of denaturation could occur on the complex organic compounds, which might be formed in extra-terrestrial region. To evaluate this denaturation process, simulated complex organic compounds will be exposed on the ISS.

The development of extra-low density aerogel (Tabata et al., 2005) is the key part of Tanpopo development for the micrometeoroid capture without damage on them. In case function of our extra-low density aerogel will be proved onboard the ISS, it will be implemented in the next generation sample return mission in the Solar system.

Our debris capture may collect many types of debris, including man-made debris, contaminated by the exhaust form the ISS, natural micrometeoroid, and micro particles ejected from Earth. We expect many valuable information could be obtained from our Tanpopo mission, and it will be open to international research community.

Arrhenius, S. (1908) Worlds in the Making - the Evolution of the Universe (translation to English by H. Borns) Harper and Brothers Publishers, New York.

Crick, F. (1981) Life Itself. Simon \& Schuster, New York.

Tabata, M., Adachi, I., Fukushima, T., Kawai, H., Kishimoto, K., Kuratani, A., Nakayama, H., Nishida, S., Noguchi, T., Okudaira, K., Tajima, T., Yano, H., Yokogawa, H., and Yoshida, H.(2005). Development of Silica Aerogel with Any Density, In IEEE Nuclear Sci. Symp. Conf. Record, pp. 816-818.

Yamagishi A., Yano, H., Okudaira, K., Kobayashi, K., Yokobori, S., Tabata, M., and Kawai, H. (in press). TANPOPO: Astrobiology Exposure and Micrometeoroid Capture Experiments on the EUSO. To appear in Symposium Proceedings of "Astronomy and Astrophysics of Extreme Universe"

Yang, Y., Itahashi, S., Yokobori, S., and Yamagishi, A. (in press)

E-mail: mita@fit.ac.jp

\section{Micro FT-IR Spectroscopic Analysis of Modern and Proterozoic Prokaryotic Fossil: Evidence of Existence of Lipids in Proterozoic Prokaryote?}

Motoko Igisu ${ }^{1}$, Yuichiro Ueno ${ }^{1}$, Mie Shimojima ${ }^{1}$, Satoru Nakashima ${ }^{2}$, Hiroyuki Ohta ${ }^{1}$, Shigenori Maruyama ${ }^{1}$

${ }^{1}$ Tokyo Institute of Technology; ${ }^{2}$ Osaka University

Carbonaceous membrane structure is one of the fundamental characteristics of Precambrian prokaryotic fossils (e.g. Schopf and Walter, 1983; Buick, 1990). However, there is no direct information on what kind of components constructed ancient microbial cellular membrane structures, while molecular fossils on cellular membrane have been reported in the previous studies on bulk analysis of extracted organic materials (e.g. Brocks et al., 2003). Here we report micro Fourier Transform Infrared (FT-IR) spectroscopic observations of modern cyanobacteria in comparison with those of extremely well-preserved Proterozoic prokaryotic fossils (Igisu et al., 2006) which are morphologically recognized as cyanobacteria (e.g. Barghoorn and Schopf, 1965).

A series of micro FT-IR measurements of modern cyanobacterial cells (Synechocystis, sp. PCC6803) and their constituents (membrane fraction, soluble fraction, and lipid 
fraction) have been conducted in order to examine the origin of functional characteristics retained in Proterozoic prokaryotic fossils from 850 Ma Bitter Springs Formation and $1900 \mathrm{Ma}$ Gunflint Formation. The whole cell and fraction samples on $\mathrm{CaF}_{2}$ disks were measured at room temperature by micro FT-IR, then were isothermally heated at 250$350^{\circ} \mathrm{C}$ under atmospheric and low-oxygen conditions. Thermally degradated samples were measured at room temperature after the heating experiments.

The bands at 2,960 $\mathrm{cm}^{-1}$ (aliphatic $\left.\mathrm{CH}_{3}\right), 2,925 \mathrm{~cm}^{-1}$ (aliphatic $\left.\mathrm{CH}_{2}\right), 1,650 \mathrm{~cm}^{-1}(\mathrm{C}=\mathrm{O}$ : amide I), and $1,540 \mathrm{~cm}^{-1}$ (CNH: amide II) are typically observed in the whole cell, the membrane fraction, and the soluble fraction, and those at $2,960 \mathrm{~cm}^{-1}$ (aliphatic $\mathrm{CH}_{3}$ ), $2,925 \mathrm{~cm}^{-1}$ (aliphatic $\mathrm{CH}_{2}$ ) are typically observed in the lipid fraction. The $\mathrm{CH}_{3} / \mathrm{CH}_{2}$ and $\mathrm{CNH} / \mathrm{CH}_{2}$ absorbance ratios reveal that each fraction can be roughly distinguished, indicating that these ratios reflect its chemical structures such as aliphatic chain length and relative amount of protein to aliphatic components. Our results show that the aliphatic $\mathrm{CH}$ moieties $\left(\mathrm{CH}_{3} / \mathrm{CH}_{2}\right.$ absorbance ratios) of Proterozoic prokaryotic fossils are similar to those of modern lipid fraction rather than other fractions. This indicates that by Proterozoic era prokaryotes might have already possessed lipid-like membranes similar to modern cells. Moreover, our preliminary results show that modern Bacteria and Archaea seem to be able to be distinguished in particular based on the $\mathrm{CH}_{3} / \mathrm{CH}_{2}$ absorbance ratio. Although micro FT-IR measurements of more kinds of modern Bacteria and Archaea are currently in progress, these results may show that prokaryotic fossils observed in this study are regarded molecular-spectroscopically as well as morphologically as Bacteria.

Barghoorn, E. S., and Schopf, J. W. (1965). Microorganisms from the Late Precambrian of Central Australia. Science, 150: 337-339.

Brocks, J. J., Buick, R., Logan, G. A., and Summons, R. E. (2003). Composition and syngeneity of molecular fossils from the 2.78 to 2.45 billion-year-old Mount Bruce Supergroup, Pilbara Craton, Western Australia. Geochimica et Cosmochimica Acta, 67: 4289-4319.

Buick, R. (1990). Microfossil recognition in Archean rocks: An appraisal of spheroids and filaments from a 3500 M.Y. Old Chert-Barite Unit at North Pole, Western Australia. Palaios, 5: 441-459.

Igisu, M., Nakashima, S., Ueno, Y., Awramik, S. M., and Maruyama, S. (2006). In situ infrared microspectroscopy of 850 million-year-old prokaryotic fossils. Applied Spectroscopy, 60: 1111-1120.

Schopf, J. W. and Walter, M. R. (1983). Archean microfossils: new evidence of ancient microbes. In Schopf, J. W. editor, Earth's Earliest Biosphere, Its Origin and Evolution Archean microfossils, pages 214-239. Princeton University Press.

E-mail: igisu.m.aa@m.titech.ac.jp

\section{Exobiological Application of Spore Dosimeter in Studies Involving Solar UV Radiation}

Pabulo Henrique Rampelotto ${ }^{1}$, Marcelo Barcellos da Rosa ${ }^{1}$,Nelson Jorge Schuch ${ }^{1}$, Nobuo Munakata $^{2}$

${ }^{1}$ Exobiology and Biosphere Laboratory, Southern Regional Space Research Center INPE/ MCT; ${ }^{2}$ Faculty of Science, Rikkyo University

The understanding of photobiological processes under different climates like space, early Earth, Mars and the consequences of the ozone role as a protector for the 
biosphere from UV solar emissions is a very important aspect in terms of the comprehension of the life evolution (Rontó et al. 2004). Consequently, recent studies have trying to understand what are the possible adaptation concepts and technologies of biological UV dosimetry, when developed for applications under climates like space and Mars surface. In this context, characteristics as a high resistance of bacterial spores to extreme conditions under extraterrestrial environments are required (Nicholson et al. 2000).

A biosensor based in the spore inactivation doses (SID) of Bacillus subtilis strain TKJ6312 has been applied in the monitoring of the UV and the results compared with UV data obtained by Brewer Spectrophotometers at the INPE's Southern Space Observatory (SSO, 29.4 $\mathrm{S}, 53.8^{\circ} \mathrm{W}$ ), South of Brazil. Due to the deficiency in both DNA repair mechanisms, Nucleotide Excision Repair (NER) and Spore Photoproduct Lyase (SP lyase), this strain is sensible to UVR and maintain the resistant for others environment conditions (Munakata et al. 2000).

The biological dosimetry fulfills the criterions established by BIODOS project from the European Commission to be applied as UV-biosensor including its simplicity, facility of use and transport, long term storage and action spectrum with a good resolution (Schuch et. al. 2006). The high correlation index around 0.9 of the continuous monthly exposition of the biosensor, which began in 2000 at the SSO, when compared with Brewer's UV measurements, demonstrates its application for long-term monitoring of the UV biologically-effective solar radiation. Furthermore, spore's data analyses from other sites around the world agree with the UV seasonal variation data cited by the literature in terms of different and adverse environmental conditions from equatorial to higher latitudes sites (Munakata et. al. 2006).

Considering the expectations of international exobiology groups to study the spatial solar radiation under different planetary environments using biological systems the application of the Bacillus subtilis TKJ 6312 seems to be a very nice biosensor tool.

Munakata, N., Kazadzis, S., Bais, A. F., Hieda, K., Rontó, G., Rettberg, P., and Horneck, G. (2000). Comparisons of spore dosimetry and spectral photometry of solar UV radiation at four sites in Japan and Europe. Photochemistry and Photobiology, 72: 739745.

Munakata, N., Cornain, S., Kanoko, M., Mulyadi, K., Lestari, S., Wirohadidjojo, W., Bolsee, D., Kazadzis, S., Schuch, N. J., Casiccia, C., Kaneko, M., Liu, C. M., Jimbow, K., Saida, T., Nishigori, C., Ogata, K., Nonaka, S., Hieda, K., and Ichihashi, M. (2006). Biological monitoring of solar-UV radiation at 17 sites in Asia, Europe and South America from 1999 to 2004. Photochemistry and Photobiology, 82: 689-694.

Nicholson, W. L., Munakata, N., Horneck, G., Melosh, H. J., and Setlow, P. (2000). Resistance of Bacillus endospores to extreme terrestrial and extraterrestrial environments. Microbiology and Molecular Biology Reviews, 64:548-572.

Rontó, G., Bérces A., Fekete, A., Kovács, G., Gróf, P., and Lammer, H. (2004). Biological UV dosimeters in simulated space conditions. Advances in Space Research, 33: 1302-1305.

Schuch, A. P., Guarnieri, R. A., Rosa, M. B., Pinheiro, D. K., Munakata, N., and Schuch, N. J. (2006). Comparisons of biologically effective doses of solar UV-radiation determined with spore dosimetry and spectral photometry in 2000-2003 at Southern Space Observatory, Brazil. Advances in Space Research, 37: 1784-1788.

E-mail: pabulo@lacesm.ufsm.br 


\section{First Results from Mars Simulator LISA}

R. Visentin ${ }^{1,2}$, G. Bertoloni ${ }^{2}$, M. D’Alessandro ${ }^{3}$, G. Galletta $^{1}$

${ }^{1}$ Dipartimento di Astronomia, Università di Padova, Italy; ${ }^{2}$ Dipartimento di Istologia, Microbiologia e Biotecnologie Mediche, Università di Padova, Italy; ${ }^{3}$ INAF-Osservatorio Astronomico di Padova, Italy

We present the first results obtained from experiments performed with the Martian simulator LISA (Laboratorio Italiano Simulazione Ambienti, Galletta et al., 2006, 2007). The research was carried out at the University and Astronomical Observatory of Padua, Italy.

LISA environmental chamber has been designed to simulate the conditions on the surface of planet Mars (atmospheric pressure, 6-9 Mb; temperature ranging from 133 to $293 \mathrm{~K}$, atmospheric composition, 95\% of carbon dioxide; strong UV radiation).

We have studied the survival of the microorganisms exposed to the above described conditions. The microorganisms used in our experiments are bacterial strains belonging to the genus Deinococcus, and to the endospore forming genera Bacillus and Clostridium (D’Angelo, 2007).

Cellular suspensions or endospores suspensions were layered on sterile coverslip dehydrated under sterile air flux, introduced in dedicated plates and then exposed to the Martian condition inside the LISA chamber.

One of our Bacillus strains has shown a particular capability to survive in Martian conditions without screening by dust or other shields, in fact we noticed a capability to survive (as endospores suspension) at least $4 \mathrm{~h}$ and in some cases to $28 \mathrm{~h}$ of Martian conditions, in the longest experiment we performed until now.

We discuss the features of the experiments, our first results and the future tests to investigate the survival of lifeforms under Martian conditions.

D’Angelo, G., (2007). Sopravvivenza di cellule e spore batteriche esposte a condizioni ambientali estreme. BSc Thesis, Dipartimento di Scienze Matematiche, Fisiche e Naturali, Università degli Studi di Padova.

Galletta, G., Ferri, F., Fanti, G., D’Alessandro, M., Bertoloni, G., Pavarin, D., Bettanini, C., Cozza, P., Pretto, P., Bianchini, G., and Debei, S. (2006). S.A.M., the Italian Martian simulation chamber. Origins of life and evolution of the biosphere, 36: 625-627.

Galletta, G., D’Alessandro, M., Bertoloni, G., Fanti, G., Danese, E., Pelizzo, M., Ferri, F., Pavarin, D., Bettanini, C., Bianchini, G., Debei, S. (2007). LISA: Mars and the limits of life. Memorie della Società Astronomica Italiana, 78: 608-611.

E-mail: giuseppe.galletta@unipd.it

\section{Early Achaean Microenvironments and Their Microbial Inhabitants}

Frances Westall

Centre de Biophysique Moléculaire, CNRS, Orléans, France

A number of micro-environments are preserved in early Archaean terrains, including both volcanic and sedimentary lithologies. Deep water sediments and volcanics from the $3.8 \mathrm{Ga}$ Greenstone Belt are unfortunately too metamorphosed to contain unambiguous traces of life but there are numerous volcanic and shallow water sedimentary environments that are very 
well preserved in the $\sim 3.5$ Ga Barberton and Pilbara Greenstone Belts. Endolithic habitats in the rinds of pillow basalts have been described by Furnes et al. (2004, 2007), Wacey et al. (2006), and McLoughlin et al. (2007) whereas macroscopic stromatolites on a carbonate platform in the North Pole Dome have been studied by Allwood et al. (2006). I will concentrate on macro and microscopic habitats in volcanic sedimentary environments from two formations, the $3.446 \mathrm{Ga}$ Kitty's Gap Chert in the Pilbara and the $3.333 \mathrm{Ga}$ biolaminated Josefsdal Chert in Barberton. Both studies are the result of pluridisciplinary investigations involving a number of collaborations (Westall et al., 2006a, b; Westall et al., 2008). In all cases the unambiguous biogenicity and syngenicity of the microbial structures was established following the criteria outlined in the above publications and in Westall and Southam (2006).

The Kitty's Gap Chert consists of silicified volcaniclastic mud-flat sediments that presented a variety microhabitats. In the water-logged sediments, the surfaces of the volcanic particles hosted colonies of plurispecies chemolithotrophic microorganisms Westall et al., 2006a) that also excavated tunnels in the surfaces of some volcanic grains (Foucher et al., 2008). Very fine-grained layers of volcanic dust also hosted pockets of chemolithotrophs. An exposed, partially cemented and stabilised surface on these mud-flat sediments was coated by small gravel-sized particles of pumice that were partially embedded in the underlying sediment before being submerged and coated with a layer of sedimented volcanic dust. Scoriaceous pores in the pumice hosted chasmolithic colonies whereas a delicate, incipient biofilm containing a consortium of different microorganisms formed on the stabilised sediment surface. The microfossils include two types of coccoids $\sim 0.5$ and $0.8 \mu \mathrm{m}$ size, $\sim 0.25 \mu \mathrm{m}$ diameter filaments (10 $\mu \mathrm{m}$ long), $1 \mu \mathrm{m}$ long rods, and EPS.

Part of the Josefsdal Chert consists of biolaminated sediments deposited in very shallow water conditions (Westall et al., 2006b, 2008). The rhythmic black and white laminations represent microbial mat layers interspersed with volcaniclastic sediments. Early diagenetic silicification of the mats ensured excellent preservation of the delicate wispy wavy carbonaceous layers. On one surface a photosynthetic microbial mat is perfectly preserved in $3 \mathrm{D}$. Formed by streamlined $0.25 \mu \mathrm{m}$ thick filaments $(10 \mu \mathrm{m}$ long), the mat was periodically exposed to desiccating conditions and evaporate mineral precipitation. HRTEM, SEM, synchrotron and nanoSIMS investigations reveal compositional and structural variability within the $5 \mu \mathrm{m}$ thick mat that is identical to that found in modern photosynthesising mats: internally the mat is partially calcified by micrite, probably due to the activity of sulphate reducing bacteria (Westall et al., 2008).

Allwood A. C., et al., 2006. Stromatolite reef from the Early Archaean era of Australia,. Nature, 441, 714-718.

Foucher, F. and Westall, F., 2008. An early Archaean sediment an analogue meteorite from noachian Mars. In prep.

Furnes, H., N.R. Banerjee, K. Muehlenbachs, H. Staudigel, M. de Wit (2004), Early Life Recorded in Archean Pillow Lavas, Science, 304, 578-581.

Furnes, H., 2007. Comparing petrographic signatures of bioalteration in recent to Mesoarchean pillow lavas: Tracing subsurface life in oceanic igneous rocks. Precambrian Research, 158, 156-176.

McLoughlin, N., et al., (2007. Formulating Biogenicity Criteria for Endolithic Microborings on Early Earth and Beyond. Astrobiology 7, 10-26.

Wacey, D., et al., 2006. The 3.4 billion-year-old Strelley Pool Sandstone: a new window into early life on Earth. Int. J. Astrobiology 5, 333-342. 
Westall F, et al., 2006. Implications of a 3.472-3.333 Ga-old subaerial microbial mat from the Barberton greenstone belt, South Africa for the UV environmental conditions on the early Earth. Phil. Trans. Roy. Soc. Lond. Series B., 361, 1857-1875.

Westall, F., et al., 2006. The 3.466 Ga Kitty's Gap Chert, an Early Archaean microbial ecosystem. In Processes on the Early Earth (W.U. Reimold \& R. Gibson, Eds.), Geol. Soc. Amer. Spec Pub., 405, 105-131

Westall, F. \& Southam, G. 2006. Early life on Earth. In Archean Geodynamics and Environments (K. Benn, et al. Eds.). pp 283-304. AGU Geophys. Monogr., 164.

Westall, F. et al., 2008. Vertical geochemical profiling across a $3.33 \mathrm{Ga}$ microbial mat from Barberton. 39th Lunar and Planetary Sciences Conference, Houston, March. Abstr.1636

Westall, F., 2008. Morphological biosignatures in terrestrial and extraterrestrial materials. Space Science Reviews, 135, 95-114.

E-mail: westall@cnrs-orleans.fr

\section{TANPOPO: Astrobiology Exposure and Micrometeoroid Capture Experiments-For Understanding Survival Possibility of Microorganisms in Space}

Shin-ichi Yokobori ${ }^{1}$, Akihiko Yamagishi $^{1}$, Yinjie Yang $^{1}$, Kenta Fujisaki ${ }^{2}$, Hirofumi Hashimoto $^{3}$, Hideyuki Kawai ${ }^{4}$, Kensei Kobayashi ${ }^{2}$, Hajime Mita ${ }^{5}$ Kyoko Okudaira ${ }^{3}$, Makoto Tabata ${ }^{4}$, Hajime Yano ${ }^{3}$, Masamichi Yamashita ${ }^{3}$, Yoshitaka Yoshimura ${ }^{6}$, TANPOPO WG

${ }^{1}$ Department of Molecular Biology, Tokyo University of Pharmacy and Life Science; ${ }^{2}$ Graduate School of Engineering, Yokohama National University; ${ }^{3}$ Institute of Space and Astronautical Science, Japan Aerospace Exploration Agency; ${ }^{4}$ Graduate School of Science, Chiba University; ${ }^{5}$ Faculty of Engineering, Fukuoka University; ${ }^{6}$ Faculty of Agriculture, Tamagawa University.

Microbes have been collected at high altitude using balloons, aircraft and meteorological rockets since 1936. Spore forming fungi, spore forming Bacilli, and Micrococci (probably Deinococci) have been isolated in these experiments. Spores and Deinococci are known by their extremely high resistance to UV, gamma ray, and other radiation. It is not clear how could those microbes be ejected up to such high altitude. If the microbes are found present even at the higher altitudes of low earth orbit, the fact would endorse the possibility of interplanetary migration of terrestrial life.

On the other hand, for the origin of life on Earth emerged within a short period after the end of heavy bombardment, Panspermia hypotheis has been proposed (e.g. Arrhenius 1908; Crick 1981). Recent findings of the Martian meteorite suggested possible existence of extraterrestrial life, and possible interplanetary migration of life as well.

TANPOPO, Japanese name of dandelion, is a plant species, whose seeds with floss are spread by wind. We propose this mission to examine possible interplanetary migration of microbes, organic compounds and meteoroids on Japan Experimental Module (JEM) of the International Space Station (ISS) (Yamagishi et al., in press). Ultra low-density aerogel will be used to capture micrometeoroid and space debris. Particles captured by aerogel will be analyzed after the initial inspection of the gel and tracks. Careful curation of the tracks in the aerogel will provide information on the size and velocity of debris captured. The particles will be characterized in terms of mineralogical, organic and microbiological properties. Aerogels are ready for production in Japan. All the analytical techniques are 
ready to conduct the TANPOPO mission. It was accepted as a candidate experiments on Exposed Facility of ISS-JEM.

In this paper, we discuss current status of exposure/capture experiments of microorganisms in the TANPOPO mission.

Arrhenius, S. (1908) Worlds in the Making-the Evolution of the Universe (translation to English by H. Borns) Harper and Brothers Publishers, New York.

Crick, F. (1981) Life Itself. Simon \& Schuster, New York.

Yamagishi A., Yano, H., Okudaira, K., Kobayashi, K., Yokobori, S., Tabata, M., and Kawai, H. (in press). TANPOPO: Astrobiology Exposure and Micrometeoroid Capture Experiments on the EUSO. To be appeared in Symposium Proceedings of "Astronomy and Astrophysics of Extreme Universe"

E-mail: yokobori@1s.toyaku.ac.jp 


\title{
Habitability and Extremophiles
}

\section{Halophile Archeabacteria at Different UV Doses: An Experiment for the UV Limits of Life}

\author{
X. C. Abrevaya ${ }^{1}$, H. P. Adamo ${ }^{2}$, P. J. D. Mauas ${ }^{1}$
}

${ }^{1}$ Instituto de Astronomía y Física del Espacio (IAFE)-UBA-CONICET; ${ }^{2}$ Instituto de Química y Fisico-Química Biológicas (IQUIFIB)-FFyB-UBA. Buenos Aires, Argentina.

Life is particularly vulnerable to ultraviolet radiation (UV). High exposure to UVC (wavelengths $<290 \mathrm{~nm}$ ) is lethal to most of the terrestrial biological systems and can set a limit for the development of extraterrestial life (Buccino et al., 2006). Halophile archeabacteria are known inhabitants of halites and ancient evaporites in Earth. Since evaporites have been detected in Martian meteorites (Zolensky et al. 1999, Whitby et al. 2000), these organisms are proposed as plausible inhabitants of Mars-like planets or other extrasolar planets (Stan-Lotter et al. 2004). Moreover, because halophiles are exposed to intense solar UV radiation in their natural environment they are generally regarded as relatively UV tolerant.

We examine the effect of UVC on the haloalcalophile archea Natrialba magadii. To this end cultures of $N$. magadii were grown to mid-exponential phase (around $\mathrm{OD}_{600}=1$ ) at $37^{\circ} \mathrm{C}$, in rich media ( $\mathrm{pH} \mathrm{10}$ ) containing (in $\mathrm{g} / \mathrm{l}$ ): yeast extract, $20 ; \mathrm{NaCl}, 200 ; \mathrm{Na}_{2} \mathrm{CO}_{3}, 18.5$; and exposed to a Phillips $15 \mathrm{~W} \mathrm{Hg}$ lamp $254 \mathrm{~nm}$ with constant mixing. Aliquots of the irradiated culture were withdrawn after different irradiation times, and the effect of the UV treatment was assessed by diluting the sample and following the changes of the growth kinetics in media of identical composition. Growth was monitored by increasing in optical density at $600 \mathrm{~nm}$. Preliminary results show that even after significant UV damage, as judged by the absence of detectable growth for more than $30 \mathrm{~h}$, the surviving cells were able to resume growth with nearly normal kinetics.

Buccino, A. P., Lemarchand, G. A., Mauas P.J.D. (2006) Ultraviolet radiation constraints around the circumstellar habitable zones. Icarus, Volume 183, Issue 2, p. 491-503.

Cockell, C.S. (1998). "Biological effect of High Ultraviolet Radiation on early Earth-a Theorical Evaluation”. J. Theor. Biol., 193, 717.

Lindberg, C. and Horneck, G. (1991). “Action spectra for survival and spore photoproduct formation of Bacillus subtilis irradiated with short-wavelength (200-300 nm) UV at atmospheric pressure and in vacuo". J. Photochem. Photobiol. B: Biol., 11: 69-80.

Stan-Lotter, H., Radax, C., McGenity, T.J., Legat, A., Pfaffenhuemer, M., Wieland, H., Gruber, C., Denner, E.B.M. (2004). From Intraterrestrials to Extraterrestrials-Viable Haloarchaea in Ancient Salt Deposits. In: Halophilic Microorganisms. Ventosa A. (Ed.), Springer Verlag, Berlin, Heidelberg, New York, pp. 89-102.

Toupance, G., Bossard, A., and Raulin, F., (1977). "Far UV irradiation of model prebiotic atmospheres". Origins of Life, 8: 259-266.

Whitby, J., Burgess, R., Turner, G., Gilmour, J., Bridges, J. (2000). "Extinct 129I in Halite from a Primitive Meteorite: Evidence for Evaporite Formation in the Early Solar System", Science, 288, 1819-1821.

Zolensky, M. E., Bodnar, R. J., Gibson, E. K., Jr., Nyquist, L. E., Reese, Y., Shih, C.-Y., Wiesmann, H. (1999). "Asteroidal water within fluid inclusion-bearing halite in an H5 chondrite, Monahans" (1998), Science, 285: 1377-1379.

E-mail: abrevaya@iafe.uba.ar 


\title{
A Statistical Study Through Time of Some Meteorological Variables Measured Around the Treeline of Pico de Orizaba
}

\author{
L. Cruz-Kuri ${ }^{1}$, C. McKay ${ }^{2}$, R. Navarro-González ${ }^{3}$
}

${ }^{1}$ Instituto de Ciencias Básicas. University of Veracruz. MEXICO $;{ }^{2}$ Ames Research Center. NASA. USA; ${ }^{3}$ Instituto de Ciencias Nucleares. UNAM. MEXICO

We are interested in treelines because of Mars and the possibility that in the future it might be habitable. We think that it had water in the past, maybe biology too; today it has no liquid water, but we think that in the future it might have liquid water again. Some of the astrobiology questions address to the potential for survival and the evolution of life beyond the planet of origin and in particular to the question if life could adapt to Mars. Perhaps it could be habitable for plants. The connection with Mars and treeline is natural: today Mars can be compared to the top of a mountain, very cold and very dry, nothing can grow there, but the process of making Mars habitable, in a sense, can be compared, as it was made explicit in a paper several years ago, with a metaphor of coming down a mountain: as one comes down, the first thing one notices is the absence of ice, then fair ground, next microbes and then the presence of plants and trees; so the study of trees is a key step and this takes us to Pico de Orizaba $\left(19^{\circ} \mathrm{N}\right)$ which has the highest treeline. Why is this so? This is one several big questions. One hypothesis refers to climate, another one to biology. We have climate data, microbiology data and soil data.

This is a preliminary report about statistical analyses performed to multivariate time series of some meteorological variables measured around the treeline of Pico de Orizaba. The data span a period of almost 10 years. The study is just an aspect of a series of approaches with the goal of gaining a better understanding of treelines in our planet and its possible relation to adaptability of life in other worlds, in particular to Mars.

Cruz-Kuri, L., McKay, C. And Navarro-González, R. (2004). Some Statistical Aspects Related to the Study of Treelines in Pico de Orizaba. COLE. Volume 7. Cellular Origin, Life in Extreme Habitats and Astrobiology. J. Seckbach et al (eds.), Life in the Universe, 223-224. Kluwer Academic Publishers.

Körner, C. (2003). Functional Plant Ecology of High Mountain Ecosystems. SpringerVerlag. Berlin, Heidelberg, New York. Third Edition.

McKay, C. (2008). Astrobiologic relevance of Pico de Orizaba for terraforming Mars. Workshop on the Astrobiology of Pico de Orizaba. Instituto de Ciencias Nucleares, UNAM.

E-mail: kruz1111@yahoo.com.mx

\section{Survival of Methanogens Following Desiccation at Mars Surface Pressure}

Timothy A. Kral ${ }^{1,2}$, Travis S. Altheide ${ }^{1}$, Adrienne E. Lueders ${ }^{2}$

${ }^{1}$ Arkansas Center for Space and Planetary Sciences; ${ }^{2}$ Department of Biological Sciences, University of Arkansas, Fayetteville, 72701.

The relatively recent discoveries that liquid water most likely existed on the surface of Mars (Squyres et al., 2004) and that methane currently exists in the Martian atmosphere 
(Formisano et al., 2004; Krasnopolsky et al., 2004) have fueled the possibility of extant or extinct life on Mars. One possible explanation for the methane in the Martian atmosphere would be the presence of methanogens in the subsurface. Methanogens are microorganisms in the domain Archaea that can metabolize molecular hydrogen as an energy source, carbon dioxide as a carbon source, and produce methane. One important factor is the arid nature of Mars. Life as we know it requires liquid water, and if it is present on Mars, it may be seasonal just as it is at some locations on our home planet.

Here we report on research designed to determine if certain species of methanogens can survive desiccation at Mars surface pressure of 6 mbar, both in a Mars soil simulant, JSC Mars-1 (Kral et al., 2004), and as naked cells. Methanosarcina barkeri, Methanobacterium formicicum, Methanococcus maripaludis and Methanothermobacter wolfeii were grown in their respective growth media in anaerobic culture tubes. Some of these cultures were added to a sterile Mars soil simulant, JSC Mars-1, some were kept in their sealed anaerobic culture tubes in liquid media, and some were centrifuged followed by removal of the supernatant media. The tubes, with syringe needles inserted through their rubber stoppers, were placed into an environmental simulation chamber. The chamber was sealed and evacuated down to 6 mbar resulting in desiccation of all of the cultures. Desiccation time varied from a few minutes for cultures that were centrifuged to two days for tubes containing liquid media. Following 60 days at 6 mbar, the tubes were removed from the chamber, rehydrated, and placed under ideal growth conditions for the respective methanogens. Cultures of all four organisms that were centrifuged and then maintained as naked cells at 6 mbar demonstrated substantial methane production $(50 \%$ or greater), while cultures in JSC Mars-1 demonstrated much less if any methane production. Of the cultures that took two days to desiccate, only $M$. formicicum demonstrated substantial methane production (approximately $40 \%)$.

In another experiment where the methanogens were desiccated at 6 mbar for 90 days, similar results were observed except for M. maripaludis, which did not survive as naked cells or on JSC Mars-1.

In order to compare desiccation effects at 6 mbar to those at Earth surface pressure, similar experiments were conducted with naked cells of the four methanogenic species in a desiccator located within an anaerobic chamber at ambient pressure. Following 90 days of desiccation, M. barkeri and M. formicicum produced substantial methane. M. wolfeii demonstrated very little methane production following 15 days of desiccation, while M. maripaludis didn't show much methane production after any desiccation period.

Formisano, V., Atreya, S., Encrenaz, T., Ignatiev, N., and Giuranna, M. (2004) Detection of methane in the atmosphere of Mars. Science 306, 1758-1761.

Kral, T.A., Bekkum, C.R., and McKay, C.P. (2004) Growth of methanogens on a Mars soil simulant. Origins Life Evol. Biosphere 34, 615-626.

Krasnopolsky, V.A., Maillard, J.P., and Owen, T.C. (2004) Detection of methane in the martian atmosphere: evidence for life? Icarus 172, 537-547.

Squyres, S.W., Grotzinger, J.P., Arvidson, R.E., Bell, J.F., Calvin, W., Christensen, P.R., Clark, B.C., Crisp, J.A., Farrand, W.H., Herkenhoff, K.E., Johnson, J.R., Klingelhofer, G., Knoll, A.H., McLennan, S.M., McSween, H.Y., Morris, R.V., Rice, J.W., Rieder, R., and Soderblom, L.A. (2004) In situ evidence for an ancient aqueous environment at Meridiani Planum, Mars. Science 306, 1709-1714.

E-mail: tkral@uark.edu 


\section{Deinococcus radiodurans Survives an Extreme Experiment Simulating the Migration Period of the Panspermia Hypothesis}

Ivan Lima ${ }^{1}$, Sergio Pilling ${ }^{2}$, Arnaldo Naves de Brito ${ }^{2}$, João Alexandre Barbosa ${ }^{2}$, Álvaro Leitão $^{1}$, Claudia Lage ${ }^{1}$

${ }^{1}$ Carlos Chagas Filho Biophysics Institute (IBCCF); ${ }^{2}$ Brazilian Synchrotron Light Laboratory (LNLS)

Extremophile microorganisms are living beings adapted to environmental conditions extremely harsh for the most kind of known organisms (Cox \& Battista, 2005; Rothschild $\&$ Mancinelli, 2001). Due to their peculiar properties, some of these microorganisms would be unique regarding the hypothetical capacity to survive in other places of the solar system, such as Mars, Venus and moons of the giant planets, such as Titan and Europa.

In an attempt to simulate the possible effects of an interplanetary migration process, known as Panspermia (Horneck et al., 2002), particularly those resulting from solar radiation, cells of Deinococcus radiodurans were prepared according to Saffary et al. (2002), lyophilized and exposed to several doses of ultraviolet and vacuum-ultraviolet using a synchrotron. The cells were irradiated using a polychromatic beam with energy range from 0.1 to $21.7 \mathrm{eV}(\lambda=12.9$ to $57.6 \mathrm{~nm})$.

Broken exponential survival curves were obtained with increasingly doses, clearly indicative of a shielding effect provided by the different types of microenvironment used to layer cells. The high survival rates under our experimental conditions including high vacuum for several days reinforces the possibility of an interplanetary transfer of bioactive material. This is the first report of live cells irradiated with a synchrotron light beam.

Cox, M. \& Battista, J. (2005). Deinococcus radiodurans - the consumate survivor. Nature Reviews Microbiology, 3, 882.

Horneck, G. (editor), Baumstark-Khan, (2002). Astrobiology: the quest for the conditions the conditions of life", Berlin, Springer.

Rothschild, L. J. \& Mancinelli, R. L. (2001) Life in extreme environments. Nature, 409, 1092.

Saffary R, Nandakumar R, Spencer D, Robb FT, Davila JM, Swartz M, Ofman L, Thomas RJ, DiRuggiero J. (2002) Microbial survival of space vacuum and extreme ultraviolet irradiation: strain isolation and analysis during a rocket flight. FEMS Microbiol Lett. 215:163-168.

E-mail: igplima@biof.ufrj.br

\section{Metanogenic Diversity Through $m c r A$ Gene in Hypersaline Conditions}

Irma Lozada-Chávez ${ }^{1}$, Lilia Montoya ${ }^{2}$, Nuria Rodriguez ${ }^{3}$, Irma Marin ${ }^{2}$, Ricardo Amils ${ }^{2,3}$

${ }^{1}$ Programa de Genómica Computacional, Centro de Ciencias Genómicas, Universidad Nacional Autónoma de Mexico, Apdo. Postal 565-A, Av. Universidad, Cuernavaca, Morelos, 62100 Mexico; ${ }^{2}$ Centro de Biología Molecular, CSIC-Universidad Autónoma de Madrid, 28049. Madrid, Spain; ${ }^{3}$ Centro de Astrobiología, INTA, 28855 Torrejón de Ardoz, Spain

Methanogenesis is one of the main metabolisms that were present in the early anoxigenic Earth's epoch (Canfield et al., 2006). Methane is the principal product originated from this 
metabolic process and it can be found in different environments, e.g., hydrothermal vents, animal rumen and sediments, and is physiological and phylogenetically confined to the methanogenic Archaea. In fact, the methanogenesis' role in the carbon cycle is especially relevant given that methanogen niches were probably dominant prior to the rise of $\mathrm{O}_{2}$ (Sleep and Bird, 2007). Two important constraints in the ecological distribution of this metabolism have been (1) redox potential and (2) sulfate concentration. Therefore, we study the methanogen community of Tirez lagoon (Spain), an athalassohaline hypersaline sabkha, which is an anoxigenic ecosystem that has been distinguished for its high sulfate concentrations. We approached an experimental technique, Denaturing Gradient Gel Electrophoresis (DGGE), focused on the identification of a methanogenic population based on band patterns from $m c r A$ gene fragments, which is known as a reliable functional gene marker for methanogenic Archaea.

The phylogenetic analysis revealed the presence of three phylotypes belonging to different taxonomic groups of methanogens: Methanoculleus genus (Methanomicrobiales Order) identified in the sediment during the flood season, and Methanohalobium and Methanolobus genera (Methanosarcinales Order), identified in both dry and flood seasons. In addition, we found a particular nutritional behavior in which the use of $\mathrm{CO}_{2}$ and $\mathrm{H}_{2}$ (hydrogenotrophic methanogenesis) as substrates is exclusively present in winter in comparison to the use of methylated compounds (methylotrophic methanogenesis), which can be identified in both dry and flooded seasons. It is possible to explain this behavior as a consequence of bioenergetic fitness where osmotic pressure (i.e. salt concentration) selects and preferentially maintains high energetic metabolisms, such as methylotropic methanogenesis.

This experimental scenario supports previous proposals regarding the development of methanogen niches in Europa; in fact, Tirez lagoon has been postulated as terrestrial analog of Europa's ocean, based on hydrogeological characteristics and on the Galileo Near Infrarred Maping Spectrometer (Prieto-Ballesteros et al., 2003).

Canfield, D.E., Rosing, M.T. and Bjerrum, C. (2006). Early anaerobic metabolisms. Phil. Trans. R. Soc., 361: 1819-1836.

Prieto-Ballesteros, O., Rodríguez, N., Kargel, J.S., González Kessler, C., Amils, R. and Fernández Remolar, D. (2003) Tirez Lake as a Terrestrial Analog of Europa. Astrobiology, 3: 863-877.

Sleep, N.H. and Bird, D.K. (2007) Niches of the pre-photosynthetic biosphere and geologic preservation of Earth's earliest biosphere ecology. Geobiology, 5: 101-117.

E-mail: ilozada@ccg.unam.mx

\section{Microbial Diversity of Tirez an Extreme Halophilic Environment, the Case of Ephemeral Conditions}

\section{José Rastoll ${ }^{1}$, Lilia Montoya ${ }^{1}$, Nuria Rodríguez ${ }^{2}$, Ricardo Amils ${ }^{1,2}$, Irma Marín ${ }^{1}$}

${ }^{1}$ Departamento de Biología Molecular. Universidad Autónoma de Madrid, 28049. Madrid, Spain; ${ }^{2}$ Centro de Astrobiología, INTA, 28855. Torrejón de Ardoz, Spain

Tirez is an inland hypersaline lagoon located in La Mancha, one of the three Iberian Peninsula endorheic arid regions. The continental climate conditions causes its physicochemical features to be ephemeral, alternating periods of waters dilution, when microbial life proliferates, followed by drought ones, when the brine precipitates generating evaporitic 
sediments (Prieto-Ballesteros et al., 2003). Tirez lagoon is chemically defined as an athalassohaline environment, since sulfate concentration can reach ten times that of chloride. Most ecological information about hypersaline systems has been generated, however, from thalassohaline systems since, generally, hypersaline communities are considered as Early Earth models. The primary productivity in these systems relies on prokaryotic microorganisms (Ley et al., 2007), and members of the Eukarya domain are absent or low abundant. In contrast, there are few studies focused on athalassohaline environments and particularly on those suffering of pronounced seasonal changes. In this context, the aim of this study was to reach a better understanding of the biological diversity present in the Tirez athalassohaline lagoon. To characterize the microbial communities inhabiting Tirez lagoon, we made use of molecular biology, as well as classical microorganisms isolation techniques. In both approaches 16S rRNA gene sequence is used as an identification and phylogenetic adscription tool. Phylotypes detected by molecular biology techniques, such as PCR, DGGE and cloning, include Halomonas sp. (Bacteria) in both dry and humid seasons; Halobacterium sp. and Halorubrum sp. (Archaea) only in the dry period and Microcoleus sp. (Cyanobacteria) in the flooded one. Isolates from flooded season were assigned to the Phylum Cyanobacteria: Oscillatoria and Leptolyngbya genera while Dunaliella was identified as the main primary producer in high osmolarity conditions $(33 \%(w / v)$ of salts) In conclusion, the euryhaline Phylum Proteobacteria was the dominant taxa during high and low salinity periods $(5.2 \%$ and $33 \%(w / v)$ of salts, respectively) and Tirez lagoon does not show significant differences, at the Phylum level, with the microorganisms found in other hypersaline lakes (see e.g., Demergasso et al., 2004).

Demergasso et al. (2004). Distribution of prokaryotic genetic diversity in athalassohaline lakes of the Atacama Desert, Northern Chile. FEMS Microbial Ecology, 48:57-69.

Ley, et al. (2006). Unexpected Diversity and Complexity of the Guerrero Negro Hypersaline Microbial Mat. Applied and Environmental Microbiology, 72:3685-3695.

Prieto-Ballesteros, et al. (2003). Tirez Lake as a Terrestrial Analog of Europa. Astrobiology, 3:863-877.

E-mail: imarin@cbm.uam.es

\title{
The Sulfur Cycle in Hypersaline Sediments Elucidated by Aps Gene Marker
}

\author{
Lilia Montoya ${ }^{1}$, Nuria Rodríguez ${ }^{2}$, Ricardo Amils ${ }^{1,2}$, Irma Marin ${ }^{1}$
}

${ }^{1}$ Centro de Biología Molecular, CSIC-Universidad Autónoma de Madrid, 28049. Madrid, Spain; ${ }^{2}$ Centro de Astrobiología, INTA, 28855 Torrejón de Ardoz, Spain

Microbial communities are deeply involved in biogeochemical cycles. Metabolic interactions in the sulfur cycle have been extensively studied, particularly in marine sediments where concentration of sulfur bearing compounds is higher than in freshwater systems (Ravenschlag, et al., 2000). However, the role of halophilic and halotolerant microorganisms in this cycle is still poorly understood. Although sequence analyses of $16 \mathrm{~S}$ rRNA gene is a generally used method to study natural microbial diversity, microorganisms involved in the sulfur cycle can be tracked using the Aps gene. Adenosine-5'phosphosulfate reductase, coded by $A p s$, is an essential enzyme of dissimilatory sulfate respiration and sulfur oxidation pathways (Meyer \& Kuever, 2008), which has been found in all sulfur reducing prokaryotes (SRP) and sulfur oxidizing bacteria (SOB) with a 
remarkably high degree of conservation, thus it is a useful functional gene marker. In this study we investigated SRB and SOB diversity in the Tirez lagoon (La Mancha, central Spain) by sequence analysis of a PCR-amplified region of the Aps gene (Deplancke, et al., 2000). Samples of DNA were obtained directly from the environmental samples or from enrichment cultures. DNA samples were used to obtain PCR-DGGE fingerprinting. Most of the Aps sequences obtained from DGGE fragments from both type of samples were closely related to Aps genes of Desulfobacterium (Deltaproteobacteria), which are complete carbon mineralizers. Some sequences branched in the tree with the sulfate reducing genera Desulfomonile, Desulfonema and Desulfotomaculum (Deltaproteobacteria). Diversity of sulfur oxidizing bacteria was represented by two genera: Thiobacillus (Betaproteobacteria) and Halochromatium (Gammaproteobacteria). This study contributes to the understanding of sulfur cycle in hypersaline ecosystems, identifying the microorganisms present in the Tirez lagoon that are involved in sulfate reduction and sulfur oxidation. The presence of Desulfobacterium sp. at high salt osmolarity conditions shows that complete mineralizers are not excluded from hypersaline environments as previously postulated by Oren (2001), being active in the sediments although at low levels.

Deplancke, B., Hristova, K. R., Oakley, H. A., McCracken, V. J., Aminov, R., Mackie, R. I. \& Gaskins, H. R. (2000). Molecular Ecological Analysis of the Succession and Diversity of Sulfate-Reducing Bacteria in the Mouse Gastrointestinal Tract. Applied and Environmental Microbiology, 66:2166-2174.

Meyer, B. and Kuever, J. (2008). Homology Modeling of Dissimilatory APS Reductases (AprBA) of Sulfur-Oxidizing and Sulfate-Reducing Prokaryotes. PLoS ONE, 3:1-16.

Oren, A. (2001). The bioenergetic basis for the decrease in metabolic diversity at increasing salt concentrations: implications for the functioning of salt lake ecosystems. Hydrobiologia, 466:61-72.

Ravenschlag, K., Sahm, K., Knoblauch, C., Jørgensen, B.B. and Amann, R. (2000). Community structure, cellular rRNA content, and activity of sulfate-reducing bacteria in marine arctic sediments. Applied and Environmental Microbiology, 66:3592-602.

E-mail: 1montoya@cbm.uam.es

\section{Adaptability of Halotolerant-Bacteria to Europa's Environment}

Horacio Terrazas ${ }^{1}$, Sandra I. Ramírez ${ }^{2}$, Enrique Sánchez ${ }^{3}$

${ }^{1}$ Facultad de Ciencias Biológicas; ${ }^{2}$ Centro de Investigaciones Químicas; ${ }^{3}$ Centro de Investigación en Biotecnología, Universidad Autónoma del Estado de Morelos, Av. Universidad No. 1001 Col. Chamilpa 62209 Cuernavaca, Morelos MEXICO

Extremophiles are distinguished by their capacity to develop basic metabolic activities in environments with physical and chemical harsh conditions where most of the mesophiles organisms cannot survive (Rothschild and Mancinelli, 2001). Halophiles are a particular type of extremophiles capable of living in moderate to high saline concentration values, extremely resistant to microgravity conditions and UV radiation exhibition, able to stay viable for long periods of time within saline crystals and with a highly specialized biochemistry (Oren, 1999). These characteristics have stimulated the study on the viability to use halophiles as models in Astrobiology studies (Dassarma, 2006), particularly for the Europan satellite environment whose main characteristic is the presence of a deep liquid water ocean rich in salts $\left(\mathrm{NaCl}, \mathrm{MgSO}_{4}\right)$ with tidal forces occurring between the ocean and 
its thick ice cover (Marion et al. 2003). The objective of this study is to evaluate the capability of halotolerant bacteria to growth on laboratory conditions analogue to those of the Europan ocean surface.

We have been conducting experiments design to test the limits for growth of halotolerant bacteria collected from a liquid industrial brine with salt contents of $6-10 \%(w / v)$ measured as $\mathrm{NaCl}$. The parameters of interest are the highest limit of salinity, and proton concentration $(\mathrm{pH})$, as well as the lowest temperature limit. After a purification process and a detailed observation of morphological characteristics, the presence of three distinct stocks identified here as T806-1, T806-2, and T806-3 was confirmed. Further biochemical and molecular tests based on $16 \mathrm{~S}$ rRNA unit allowed a more detailed classification. A $10 \%$ TSB culture medium was used in all cases and bacterial growth was monitored spectroscopically by determining the optical density at $600 \mathrm{~nm}$ (Genesys 20 Thermo Spectronic) and also by viable count tests, performed at 48 -h intervals in all cases.

The experimental conditions can be summarized as follows: each one of the three stocks was grown in a culture medium enriched with $\mathrm{NaCl}, \mathrm{MgSO}_{4}$ and $\mathrm{Na}_{3} \mathrm{PO}_{4}$ at $2 \%, 5 \%$ and $10 \% \mathrm{w} / \mathrm{v}$ concentration. The acidity of the culture medium was set at $\mathrm{pH}$ values of $2.0,5.5$ and 9.0 with a phosphate buffer. The Europa's ocean surface scenario was simulated using a hermetically isolated $100-\mathrm{mL}$ flask where $50 \mathrm{~mL}$ of the $10 \%$ TSB medium was inoculated with a combination of T806-1 and T806-3 strains and enriched with 5\% $\mathrm{NaCl}$ and $10 \%$ $\mathrm{MgSO}_{4}$ at a $\mathrm{pH}$ value of 5.5. Tests were performed introducing $50 \mathrm{mbar}$ of $5 \%, 10 \%$ and $20 \% v / v$ oxygen content balanced with argon.

Three different stocks were isolated and characterized. Two of them, T806-1 and T806-3 were perfectly able to grow in the presence of up to $10 \%$ of $\mathrm{NaCl}$ and $\mathrm{MgSO}_{4}$ and at an acidity value of 5.5. These conditions have specific relevance to the Europan ocean. Their growth, monitored spectroscopically by the optical density, showed the capability of these bacteria to adapt to high contents of salts. The halotolerant bacteria have also demonstrated their capability to resist short exposures to low temperatures (below the water freezing point), after which they continue viable. The implications of all these results in the frame of a salty Europan ocean will be presented and discussed.

Dassarma, Shiladitya, (2006). Extreme Halophiles are models for Astrobiology. Microbe, 1(3). Marion, G., Fritsen, C., Eicken, H., and Payne, M. (2003). The search for life on Europe: Limiting environmental factors, potential habitats, and Earth analogues. Astrobiology, 3 (4):785-811.

Oren, A. (1999). Bioenergetic aspects of halophilism. Microbiol. Mol. Biol. Rev. 63: 334348.

Rothschild, L. J. and Mancinelli, R. L. (2001). Life in Extreme Environments. Nature, 409: 1092-1101.

E-mail: ramirez_sandra@ciq.uaem.mx

\section{Galaxy Simulations as a Tool for Mapping Habitable Zones}

G. Vladilo ${ }^{1}$, P. Monaco ${ }^{2,1}$, G. Murante ${ }^{3}$, L. Tornatore ${ }^{2}$

${ }^{1}$ Osservatorio Astronomico di Trieste-INAF; ${ }^{2}$ Dipartimento di Astronomia, Università di Trieste; ${ }^{3}$ Osservatorio Astronomico di Torino-INAF

We simulate the evolution of a disk galaxy in a cosmological-like context by using an evolving gravitational potential which emulates the hierarchical growth of a suitable dark 
matter halo. We plan to perform such simulations with the code GADGET-2 at very high resolution, using gas particle masses ranging from $10^{4}$ to $10^{3}$ solar masses. By using a chemical evolution model that we have recently implemented in the code (Tornatore et al., 2007), we will obtain the spatial distribution of the metallicity, estimated for several elements, and of the rate of supernovae explosions at any given time of the galaxy evolution. From these data we will be able to map the temporal evolution of habitable zones in a galaxy like ours, by introducing a metallicity-dependent threshold of planet formation (Vladilo, 2004) and by parametrizing the sterilyzing effect of supernovae explosions.

Tornatore, L., Borgani, S., Dolag, K. and Matteucci, F. (2007). Chemical enrichment of galaxy clusters from hydrodynamical simulations. MNRAS, 382:1050-1072.

Vladilo, G. (2004). Dust and planet formation in the early Universe. In Seckbach, J. et al., editors, Life in the Universe, pages 167-168, Kluwer Academic Publishers

E-mail: vladilo@oats.inaf.it

\section{Adaptability of Bacillus subtilis 168 Cells to High UV Stress}

\section{Marko Wassmann, Ralf Moeller, Günther Reitz, Petra Rettberg}

German Aerospace Center (DLR), Institute of Aerospace Medicine, Radiation Biology Department, Research Group Photo- and Exobiology, Linder Hoehe, D-51147 Cologne, Germany

Previous experiments have shown that vegetative cells of Bacillus subtilis are capable to repair large amounts of DNA photolesions directly after irradiation. But no DNA repair process is error-free, leading to mutations which will be inherited to the following generations (Sung et al., 2003). In a precursory study for the space experiment ADAPT (Molecular adaptation strategies of microorganisms to different space and planetary UV climate conditions)*, cells of Bacillus subtilis 168 were continuously cultured under periodical $16.8 \mathrm{~kJ} / \mathrm{m}^{2}$-polychromatic UV irradiation at 200-400 $\mathrm{nm}$ (Wasserman et al., 2007).

Approximately 700 generations of $B$. subtilis had been periodically exposed to UV radiation. Cells evolved under UV stress were 3-fold more resistant to UV-C compared to the ancestral and equally evolved but not UV-irradiated populations. Spores of both cell types respond similar to UV irradiation and exhibit ancestor UV survival characteristics. UV-evolved cells were 7-fold more resistant to ionizing radiation than their non-UV exposed evolved relatives and ancestor, whereas no changes in the spore survival after ionizing radiation exposure of all three populations were detectable. Current investigations on the molecular mechanisms, e.g. transcriptional profiling, will allow understanding changes on the adaptation level.

Sung, H. M., Yeamans, G., Ross, C. A., and Yasbin, R. E. (2003). Roles of YqjH and YqjW, homologs of the Escherichia coli UmuC/DinB or Y superfamily of DNA polymerases, in stationary-phase mutagenesis and UV-induced mutagenesis of Bacillus subtilis. J. Bacteriol., 185:2153-2160.

Wassmann, M., Moeller, R., Nellen, J., Reitz, G., Rabbow, E., and Rettberg, P. (2007). Bacillus subtilis' ability to adapt to extreme UV stress. Int. J. Astrobiol., 6:71.

*NASA homepage-Exposure Experiment (Expose/ADAPT) http://www.nasa.gov/ mission_pages/station/science/experiments/Expose.html

E-mail: Marko.Wassmann@dlr.de 


\title{
Historical and Philosophycal Aspects
}

\section{Santiago Ramón y Cajal and His Endosymbiotic Metastructures Within Neurons}

\author{
Ulises Iturbe ${ }^{1,3}$, Juli Peretó ${ }^{2}$, Antonio Lazcano ${ }^{1}$
}

${ }^{1}$ Facultad de Ciencias, UNAM. Apartado Postal 70-407, Cd. Universitaria, Mexico, D. F., CP 04510. Mexico; ${ }^{2}$ Institut Cavanilles de Biodiversitat i Biologia Evolutiva de la Universitat de Valencia. Apartat Postal 22085, Valencia. CP 46071. España; ${ }^{3}$ Área Académica de Biología del Instituto de Ciencias Básicas e Ingeniería. Universidad Autónoma del Estado de Hidalgo. Apartado Postal 1-69 Plaza Juárez, Pachuca de Soto, Hidalgo. CP 42001. Mexico

The hardening of the cell theory during the second half of the 19th century encountered strong resistance by those that considered viruses and hypothetical organisms smaller than cells, on the one hand, and by those that were convinced that the basic traits of life were found not in complete cells but only within protoplasm, on the other. Spanish-speaking scientists were not an exception, and some of the most distinguished members in academia became engaged in this debate. It was the case of the distinguished Spanish histologist Santiago Ramón y Cajal, who proposed the existence of hypothetical living metastructures within nucleated cells, as part of a more comprehensive "cytocolonial theory" (Ramón y Cajal, 1989). His ideas were not accepted in his country nor in Latin America due to scientific prejudices and the prevalence of the hardened version of cell theory, and in other international academic circles probably because of language barriers. Eventually, however, as he matured Ramón y Cajal abandoned his initially enthusiastic critique of the cell theory and, by his discoveries, became one of its more important supporters (López-Piñero, 2006).

López-Piñero, JM (2006) Santiago Ramón y Cajal. Colección Biografías. Publicacions de la Universitat de Valencia and Editorial de la Universidad de Granada, Valencia.

Ramón y Cajal S (1989) Recollections of my life. MIT Press, Cambridge.

E-mail: ulisesi@uaeh.edu.mx

\section{Linear Temporality: A Cultural Perspective of the Origin of Life}

Ninel Valderrama-Negrn ${ }^{1}$, Sandra Ramos-Amzquita ${ }^{2}$, Sergio Ramos-Bernal ${ }^{3}$, Alicia Negron-Mendoza ${ }^{3}$

${ }^{1}$ Facultad de Filosofa y Letras; ${ }^{2}$ Facultad de Ciencias Polticas; ${ }^{3}$ Instituto de Ciencias Nucleares, Universidad Nacional Autnoma de Mexico (UNAM) Mexico, D.F. Mexico

The Aristotelian paradigm of time plays an important role in Western Modernity (14531789), in science and in the way that Western civilization perceives the origin of life. The aim of the present paper is to analyze the philosophical basis for the origin of life in Western Modernity. Our argument takes as its point of departure the idea that the Aristotelian paradigm of linear temporality influences all aspects of life, including science, even after the outcome of the scientific method. This paradigm implies a conception of time that has as main characteristics a beginning and an end, forming the idea of linear temporality. This point of view is based on the perception of human life as finite. In addition, this temporality serves as a framework in Western thinking, which is different 
from that of other cultures. Thus, the present paper argues that the Aristotelian paradigm of time is a central concept of this culture, particularly from the Western perspective of the origin of life.

The concept of linear time shapes the notion of the origin of life in Modernity. Aristotle, who represents the philosophical thinking of Western culture, created this idea of time in relation to movement. From this point of view, time is the change of state from inactivity to activity. This perception of movement shapes the paradigm of linear temporality; therefore, it creates the need for an origin. This perspective of time is the framework of reality in which the concept of the beginning of time is immersed. Taking this paradigm into account, we analyze the work and the ideology of Francesco Redi, who was the first person to seriously question the idea of spontaneous generation. However, the cultural environment of the epoch in which he lived nourished his beliefs about origins. Redi's experiments marked the context in which nature was viewed, especially in regard to the studies of the origin of life.

Aristotle (1999). Aristotle in Twenty-three Volumes. Heinmannn, London.

Bacon, F. (2004). Novum Organum. Losada, Buenos Aires.

Cecil, W. (1972). A History of Science and its relation to Philosophy and Religin. Cambridge University Press, MA.

Descartes, R. (1979). Discurso del Mtodo. Alianza, Madrid.

Gribbin, J. (2002). Historia de la Ciencia (1543-2001). Critica, Barcelona.

Heidegger, M. (1971). El Ser y el Tiempo. Fondo de Cultura Econmica, Mxico.

Olive, L. (2000). El Bien, el Mal y la Razn. Paidos, Mxico.

Platn (2003). Dilogos. Porrua, Mxico.

Reale, G. and Antiseri, D. (1983). Historia del Pensamiento Filosfico y Cientfico. Herder, Espaa.

E-mail: negron@nucleares.unam.mx and ninelvn@gmail

\section{Edmund Perrier (1844-1921), A French Naturalist Who Discussed the Idea of Chemical Evolution as Early as 1920}

\section{Florence Raulin Cerceau}

Centre Alexandre Koyré (CNRS-EHESS-MNHN-CSI-UMR 8560) Musee national d'Histoire naturelle, Paris-France

Key Words: Edmund Perrier - Chemical Evolution—Origins of Life-History of Sciences

Edmund Perrier was a zoologist and an anatomist who became Director of the National Museum of Natural History of Paris-France from 1900 to 1919. He was a specialist of the benthic fauna and also a member of the French Academy of Sciences. He contributed to popularize many zoological notions concerning anatomy, transformism, and submarine exploration. Interested in the idea of biological evolution, he was however more a supporter of Lamarck's transformism, than a strong defender of Darwin's theory.

One of his major contributions deals with the study of the Earth before the evolution of life. This book, entitled La Terre avant l'Histoire. Les Origines de la Vie et de l'Homme, was published in 1920 while the studies on the biochemical components of the living beings were rapidly developing (Paris, La Renaissance du Livre).

The first chapter of the second part of the book (this part was entitled "The Primitive Forms of Life"-Les formes primitives de la vie) deals with the Origin of Life (L'apparition 
de la vie). In this chapter Perrier proposes his own scenario on the origin of life and shows that the phenomenon of life began with a unique starting point on a primitive earth very different from today. He gives also some methodological keys to try to experiment in laboratory the first stages leading to life. Finally he points out some difficulties that are still topical nowadays.

This paper will show what innovations had been made by Perrier in the field of the emergence of life, and why his suggestions can be regarded as very close to the first scenarios of chemical evolution.

Reale, G. and Antiseri, D. (1983). Historia del Pensamiento Filosfico y Cientfico. Herder, Espaa.

E-mail: raulin@mnhn.fr

\section{Life as a Functional Concept: Functionalism as a Robust Framework for Theories and Definitions of Multi-realized Living Systems}

Olin Robus ${ }^{1,3}$, Nathan Haydon ${ }^{1,3}$, Shawn McGlynn ${ }^{1,2}$, Gordon Brittan ${ }^{3}$

${ }^{1}$ NASA Astrobiology Institute; Astrobiology Biogeocatalysis Research Center; ${ }^{2}$ Department of Chemistry and Biochemistry; ${ }^{3}$ Department of History and Philosophy, Montana State University Bozeman, MT 59717 United States

Past attempts defining life have been largely unsuccessful, due in part to a flaw common to all of these attempts. Namely, these attempts are intrinsically handicapped by their formulation within a framework that implicitly assumes life is a "Natural Kind." This characterization of life as a Natural Kind is ubiquitous, either implicitly or explicitly, in many definitions and theories of life.

We argue that the Natural Kind paradigm falsely suggests an ontological category for living systems, and hinders investigations and exploration for non-terrestrial life. Contemporary searches for non-terrestrial living systems should rely upon a theory that can accommodate multiple realizations of life in diverse contexts. The Natural Kind paradigm unnecessarily restricts the domain of potential realizations to an artificially small range of physical arrangements.

We suggest a new conceptual framework for studying living systems, the origins of life, and the resulting theories and definitions of life, generally construed. We propose that understanding life as a functional class, rather than a Natural Kind, offers a robust and fruitful framework for posing and approaching scientific and conceptual questions about living systems. It will be shown that functionalism preserves our intuitions about living systems "as-we-know-them", while providing a strong theoretic framework for encountering and identifying new and novel realizations of living systems in a variety of nonterrestrial physical contexts.

Cleland, Carol E., and Christopher F. Chyba. "Defining 'Life"' Origins of Life and Evolution in the Biosphere 32 (2002): 387-393. Pattee, H. H. "Simulations, Realizations, and Theories of Life." The Philosophy of Artificial Life. Ed. Margaret A. Boden. New York: Oxford UP, 1996. 379-393. Quine, W.V. O. "Natural Kinds." Ontological Relativity and Other Essays. New York: Columbia UP, 1969. 114-138. Schneider, Eric D., and Dorion Sagan. Into the Cool: Energy Flow, Thermodynamics, and Life. New York: University of Chicago P, 2006.

E-mail: olin.robus@gmail.com 


\title{
Study of the Opinion of University Students on the Themes of the Origin and Evolution of Life
}

\author{
Rogério F. de Souza ${ }^{1}$, Marcelo de Carvalho ${ }^{1}$, Tiemi Matsuo ${ }^{2}$, Dimas A. M. Zaia ${ }^{3}$
}

${ }^{1}$ Departamento de Biologia Geral-CCB; ${ }^{2}$ Departamento de Estatística e Matemática Aplicada-CCE; ${ }^{3}$ Laboratório de Química Prebiótica, Departamento de Química-CCE, Universidade Estadual de Londrina, 86051-990, Londrina-PR, Brazil

Teaching about the origin and evolution of life is very complex, requiring professors to have a solid training in the subject. However, currently, the complexity of these themes is not the only problem confronted by these professors. In Brazil, as in many other countries (mainly the United States), a strongly religious movement called creationism has orchestrated various steps in attempt to impose on public learning institutions a religious vision of the teaching of the origin and evolution of life. We can say that a creationist is one who rejects evolution in favor of a divine creator (Downie et al., 2000; Moore and Miksch, 2003). In view of the lack of information in the Brazilian literature on the opinion on of university students of biological evolution, a questionnaire was administered in the years 2006 and 2007 to first-year and fourth-year students in the following curricula (associate's degree and bachelor's degree): biology, philosophy, physics, geography, history and chemistry. The total number of questionnaires filled out was about 900, where it consisted of two parts; a socio-economic survey of students and 11 multiple-choice questions referring to the degree of acceptance/rejection of the themes related to the origin and evolution of the universe and life, as well as questions related to more common scientific themes. The chi-squared test was used for statistical analysis of the association between the characteristics of the students and the questions of the study. In general, we observed that an increase in the education level of the mother and father decreased significantly the degree of rejection of themes related to origin and evolution $(p<0.05)$. We noted that the schooling of the mother appeared to be more important than that of the father. However, when asked if smoking causes lung cancer, education level of the father or mother, religion and family income had no influence on the answer $(p>0.05)$, where $20 \%$ of the UEL students had doubts about the truth of this. Family income showed no influence on the acceptance or rejection of themes related to the life's origin and evolution $(p>0.05)$. A statistical analysis was also carried out taking into account the religion of the students. The students were divided into three major groups: Roman Catholics, non-Catholic Christians and others. In general, it was observed that the groups Roman Catholics and others were much more inclined toward accepting themes involving the origin and evolution of life, than the non-Catholic Christian group $(p<0.05)$. In all the curriculum areas, $40 \%$ to $65 \%$ of the students showed acceptance of biological evolution without discarding the existence of a god. That is, for many of the students, this concept does not present a conflict. When asked if science can provide reliable answers to physical, chemical and biological phenomena, we observed that family income and education level of the mother and father had more influence than religious belief. However, we can state that in general, there is a high incidence of trust in science, since we found that only $5 \%$ think that science does not provide reliable answers with regard to physical, chemical and biological phenomena. The data also demonstrate that in general there is a tendency for a greater acceptance of themes related to the origin and evolution of life in fourth-year than in first-year university students. 
Downie J. R., Barron, N. J. (2000) Evolution and religion: attitudes of Scottish first year biology and medical students to the teaching of evolutionary biology. J. Biol. Educ. 343: 139-146.

Moore R., Miksch, K. L. (2003) Evolution, creationism and the courts: 20 questions. The Science Education Review 2, 15:1-15.

E-mail: damzaia@uel.br

Open Access This article is distributed under the terms of the Creative Commons Attribution Noncommercial License which permits any noncommercial use, distribution, and reproduction in any medium, provided the original author(s) and source are credited. 